\title{
Studies of DNA Supercoiling in Vivo and in Vitro
}

\author{
David Nelson Cook \\ (Ph.D. Thesis) \\ Department of Chemistry \\ University of California \\ and \\ Chemical Biodynarnics Division \\ Lawrence Berkeley Laboratory \\ University of California \\ Berkeley, Califormia 94720
}

October 1990

This work was supported by the Director, Office of Energy Research, Office of Basic Energy Sciences, of the U.S. Department of Energy under Contract No. DE-AC03-76SF00098. 
Studies of DNA Supercoiling in Vivo and in Vitro

b y

\title{
David Nelson Cook
}

\begin{abstract}
This thesis describes a number of diverse experiments whose common theme is to elaborate some aspect of DNA supercoiling. The torsion elastic constant of DNA is measure as a function of superhelix density using the technique of picosecond Time Resolved Fluorescence Polarization Anisotropy (FPA) of intercalated ethidium bromide. The sensitivity of this technique is demonstrated by measuring the anisotropy decay of lambda DNA suspended in sucrose solutions of increasing concentration. The results agree with theories which predict that the anisotropy decay should vary with the square root of the relative viscosity. This experiment furthermore demonstrates a sensitivity of FPA to a change in torsion elastic constant of less than $10 \%$. A number of covalently closed DNA samples, ranging in superhelix density from $=-0.123$ to +0.042 , are then examined. At physiological ionic strength, the torsion elastic constant varies monotonically over this range in superhelix density from 2.28 to $1.76 \times 10^{-19} \mathrm{erg} \mathrm{cm}$.

A novel method for measuring changes in local supercoiling on a large DNA molecule is presented. The assay is sensitive to changes in supercoiling of regions of chromosomal DNA as short as 1 kilobase in length. I next address the question whether stable changes in
\end{abstract}


chromosomal supercoiling regulate anaerobic gene expression in the facultative photosynthetic bacterium, Rhodobacter capsulatus. The data show that there is no stable change in chromosomal supercoiling upon a shift from aerobic respiratory growth to anaerobic photosynthetic conditions. Further experiments, which are designed to detect transient changes in DNA supercoiling, indicate that the DNA downstream from heavily transcribed genes for the photosynthetic reaction center is relaxed or perhaps overwound upon the induction of photosynthetic metabolism. These results are interpreted in terms of the twin domain model of transcriptional supercoiling.

Finally, a plasmid system is developed for the study of transcriptional supercoiling in vivo and in vitro. Two plasmids, which contain separate genes under the control of strong, repressible promoters, are constructed. These plasmids differ only in the relative orientation, either parallel or opposed, of their two transcription units. Experiments in Escherichia coli demonstrate that positive supercoils are produced in these plasmids independent of the orientation of these genes. The trapping of positively supercoiled DNA depends on the simultaneous induction of transcription and the inhibition of DNA gyrase. The kinetics of transcription, as measured by a lac $Z$ assay, are shown to be the same for parallel and opposed constructs. Novobiocin treatment inhibits transcription in both plasmid species. 


\section{Acknowledgements}

Many people have contributed to the work presented in this thesis. Among these, John Hearst has played the most important role as research director and friend. John's enthusiastic support of my work, especially when things bogged down (as they often do), made the task more bearable. John encouraged me to develop my own scientific interests and style by giving me the freedom to work independently. I think that the greatest tribute to his ability as a research director is the number of outstanding scientists who have come from his lab and the equally large number of associates who still consider him a friend.

I have also been fortunate to work closely with a number of outstanding scientists on particular projects, each of whom has taught me something important. Greg Armstrong collaborated with me on the initial psoralen crosslinking experiments in Rhodobacter. I particularly benefited from his patience and calm demeanor. Paul Selvin introduced me to the wonderful world of fluorescence spectroscopy, from which I haven't yet quite recovered. I have learned many things, both scientific and otherwise, from Paul, and our continuing collaboration is one of the main reasons that I am excited about my future scientific research at Berkeley. Ning Pon is responsible for most of the experiments presented in Chapter 4, and I am indeed fortunate that he will not rest until these are brought to

a satisfactory end. Ning's scientific skills are superb beyond description, and his presence in the lab helps to make each day more enjoyable. Finally, Zack Ma, whose experiments are not 
presented in this thesis, is a friend and collaborator who has benefited me by continually bringing new techniques and ideas to my attention, and by his example of efficiency and skill in the lab.

One of the most rewarding aspects, for me, of scientific research is the fine people with whom I associate on a daily basis. The many individuals in and out of the Hearst group who have assisted me through discussions, by providing materials, or in giving technical advice, are too numerous to mention. In particular, however, I would like to single out Marie Alberti who, as anyone associated with the Hearst group knows, is the glue who holds everything together. Marie has done DNA sequencing for me in a number of instances during my graduate studies, and more importantly, she has performed some of the unpleasant but necessary tasks which keep the lab running. Her enthusiasm for science is contagious and has had a positive effect on me.

Finally, how do I thank Abbey, Rebecca, and Benjamin? Becky and Ben: you two have given my life focus which seems to accentuate even my pleasure in being a scientist. Abbey: your support and love are clearly the foundation for my work, and for this, I thank you. 


\section{Table of Contents}

Acknowledgements ............................................................................. ii

Chapter 1: Introduction to DNA Supercoiling

The description of supercoiled DNA …........................................... 2

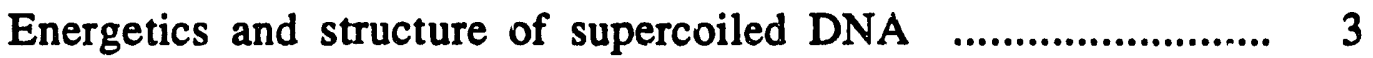

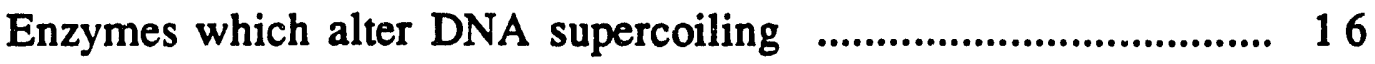

Supercoiling: effects on replication and recombination $\ldots . . .21$

Supercoiling and transcription ................................................... 24

The role of topoisomerases in the elongation step of transcription ........................................................................... 32

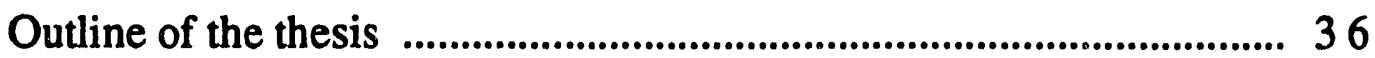

Chapter 2; Time-resolved fluorescence polarization anisotropy of superhelical DNAs

Introduction

Theory

Fluorescence anisotropy measurements f.................................. 47

The Barkley-Zimm elastic chain model ..................................... 52

The Allison-Schurr intermediate zone formula ........................ 61

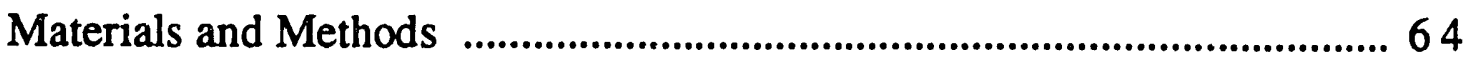

Results

Initial characterization of samples ............................................... 74

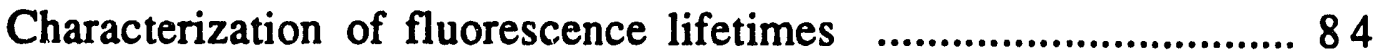


Sensitivity of anisotropy decay to changes in viscosity $\ldots . . .888$

Trrsional stiffness as a function of ionic strength ................... 98

Fluorescence anisotropy measurements of

DNA topoisomers

Discussion

Comparison of torsion constant for linear DNA with results from the literature .................................................... 104

Variation of torsion constant with superhelicity f..................1 107

Potential effects on the biological function of DNA ............... 109

The dynamics of DNA twisting ......................................................11

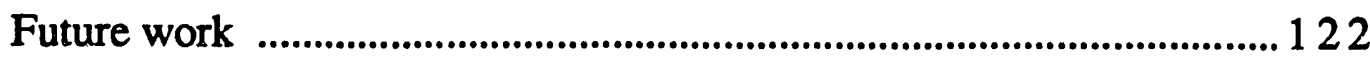

Chapter 3: Chromosomal supercoiling in Rhodobacter capsulatus during the shift from aerobic to anaerobic photosynthetic growth

Introduction

Materials and Methods

Results

An assay for local changes in chromosomal superhelicity. .136

Time-course of novobiocin action

mRNA accumulation during the shift from aerobic to anaerobic photosynthetic growth ......................................148

Chromosomal supercoiling during the induction of genes for photosynthetic metabolism

Transient changes in chromosomal supercoiling during the induction of genes for photosynthesis .............................154 


\section{Discussion}

The utility of the psoralen assay

Stable changes in unrestrained DNA supercoiling do not regulate anaerobic gene expression

The role of transcriptional supercoiling and DNA gyrase in the expression of the puf and puh operons 166

Uncertainties of the twin domain model

Chapter 4: À model system for investic ting transcriptional supercoiling

Experimental background to the twin domain model 174

The optimization of transcriptional supercoiling

Materials and Methods

Results

Construction of plasmids for maximizing transcriptional supercoiling

Construction of a strain permeable to hydrophobic solutes 194 Transcriptional supercoiling in the constructs .196

Induction of gene expression in the constructs 202

Discussion and Future Work 204

References .207 


\section{Chapter 1 \\ Introduction to DNA Supercoiling}

Supercoiling literally means the coiling of a coil. The DNA helix is supercoiled in eukaryotic and prokaryotic cells (Paulson and Laemmli, 1977; Sinden et al., 1980; Worcel and Burgi, 1972), and supercoiling is a biologically essential feature of DNA. DNA can be bound by proteins which supercoil the helix by wrapping it in a specific geometry; or DNA can be enzymatically supercoiled by underwinding of the helix, which fixes a topological constraint on the intertwining of strands. Both topological and geometric constraints determine the extent of supercoiling in cells.

Due to its importance in DNA metabolism, supercoiling has received intensive theoretical as well as experimental scrutiny. The description of supercoiling relies to a significant extent on concepts developed in mathematical topology (Ctick, 1976; Fuller, 1971; White, 1969; Glaubiger and Hearst, 1967). The structure and energetics of supercoiled DNA have also been extensively investigated (for a review, see Bauer, 1978). These studies have been greatly facilitated by the isolation of enzymes (Gellert et al., 1976; Wang, 1971) which allow for the in vitro production of supercoiled DNAs with various degrees of over- and underwinding. A host of physical, biochemical, and biological studies have investigated the role of DNA supercoiling in the function of the genetic material. The aim of this introduction is to review some of the aspects of DNA supercoiling which are essential to understanding 
the results presented later and to elaborate a background for putting these results in a more general context.

The description of supercoiled DNA. The most easily measured property of small supercoiled plasmids is the linking deficit $\Delta \mathrm{Lk}$, which derives from the concept of the linking number. The linking number $\mathrm{Lk}$ is a topological descriptor of a closed curve in space. It is a measure of the number of times a curve winds about its own axis (Fuller, 1971; White, 1969; Glaubiger and Hearst, 1967). For a curve to have a defined $\mathrm{Lk}$, its two ends must have a fixed orientation relative to one another. This means that $\mathrm{Lk}$ is not defined for a linear DNA since the ends can freely rotate, but $L k$ is a definite number for covalently closed, circular DNAs since the "ends" are joined and therefore have a fixed orientation. For large DNAs, such as eukaryotic or prokaryotic chromosomes, small regions can have a defined $\mathrm{Lk}$ if there exist topological anchors which prevent free rotation of the DNA (Paulson and Laemmli, 1977; Sinden and Pettijohn, 1981; Sinden and Pettijohn, 1982; Worcel and Burgi, 1972). An important property of $\mathrm{Lk}$ is that, for circular DNA molecules, it can have only integral values. The linking deficit, $\Delta \mathrm{Lk}$, is simply the difference between the actual $\mathrm{Lk}$ of a molecule and the value, $\mathbf{L k}_{\mathbf{o}}$, for a similar molecule whose torsional stress is relaxed under the same solution conditions: $\Delta \mathrm{Lk}=\mathrm{Lk}-\mathrm{Lk}_{\mathbf{0}}$.

An intuitive understanding of $\mathrm{Lk}$ can be gained by a simple thought experiment. To determine $L k$ for a particular molecule, imagine flattening the DNA, causing it to lie entirely in a plane. Under these circumstances, $L k$ is equal to the number of times one 
strand winds about the other; or alternatively, $L k$ is equal to the number of times one strand winds about the helix axis. Lk must be an integer, since by necessity the end point is the same as the starting point.

A more rigorous definition of $\mathbf{L k}$, which takes into account the orientation of the crossing of strands, utilizes the concept of the spanning surface (Fig 1.1). Imagine a surface bounded entirely by the helix axis. Since the DNA is not constrained to lie in a plane, this surface can exist in three dimensions. One of the DNA strands winds about the helix axis, piercing the surface each time it passes through a complete turn. A value, either +1 or -1 , can be assigned to each of these crossings depending on the orientation of the DNA strand with respect to the surface, using the following protocol: First, the helix axis is assigned an arbitrary direction and is labeled with an arrow; then the DNA strand is also labeled with an arrow parallel to the the one on the helix axis (Fig 1.1). According to the convention of the right hand rule, the spanning surface now has distinguishable sides. The fingers of the right hand curl about the helix axis parallel to the direction marked by the arrows; the extended thumb points in the upward direction, labeled with a bold arrow in Fig 1.1. If the DNA strand pierces the spanning surface from above, opposite to the bold ariow, then the crossing is assigned a value of -1 (Fig 1.1A). If the DNA strand pierces the spanning surface from below, parallel to the bold arrow, then the crossing is assigned a value of +1 (Fig 1.1B). The signed sum of the nodes equals $L k$ for an arbitrary curve. This definition of $\mathrm{Lk}$ is independent of the initial direction assigned to the 
Fig 1.1 The calculation of $\mathrm{Lk}$ using a spanning surface. The orientation of the spanning surface, defined by the helix axis, is demarcated by arrows along the border of the surface; the DNA strand is arbitrarily marked with a parallel arrow. The bold arrow in the upper part of the figure points in the direction defined by the right hand rule, as described in the text. (A) Determination of $\mathrm{Lk}$ for a left-handed helix. (B) Determination of $\mathrm{Lk}$ for a right-handed helix. The right-handed helix is analogous to B-form DNA. 


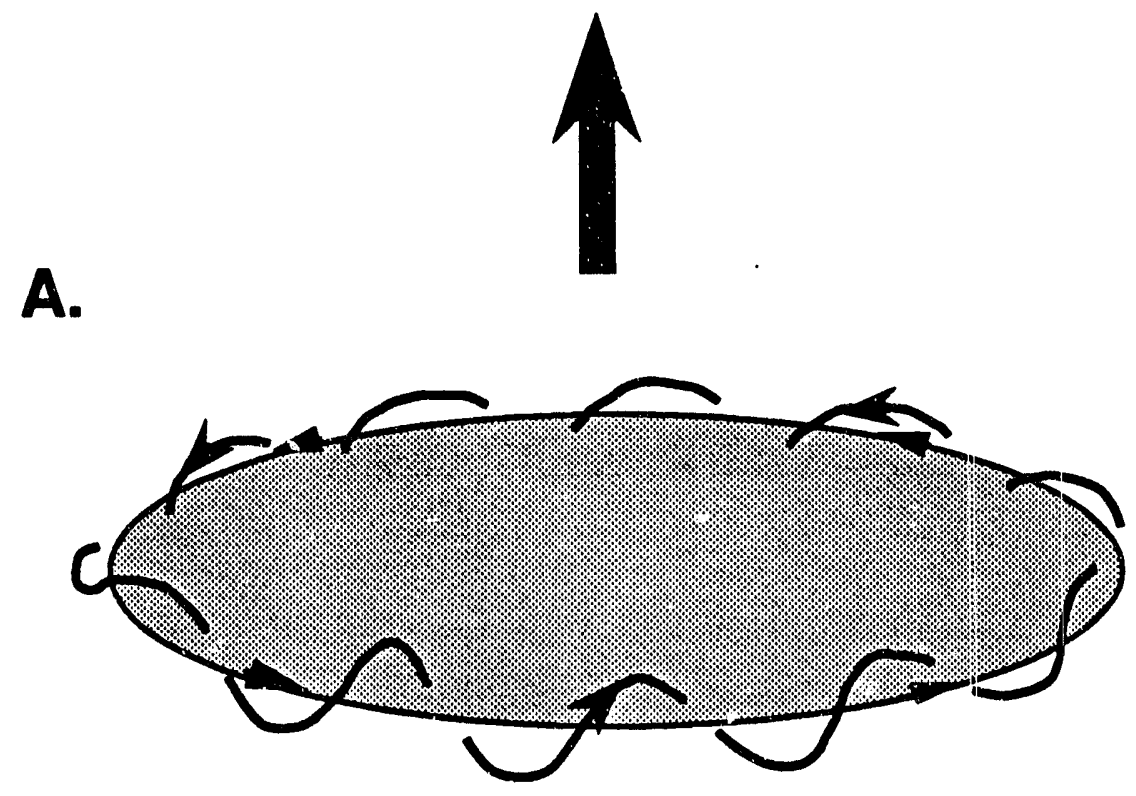

$L k=-10$

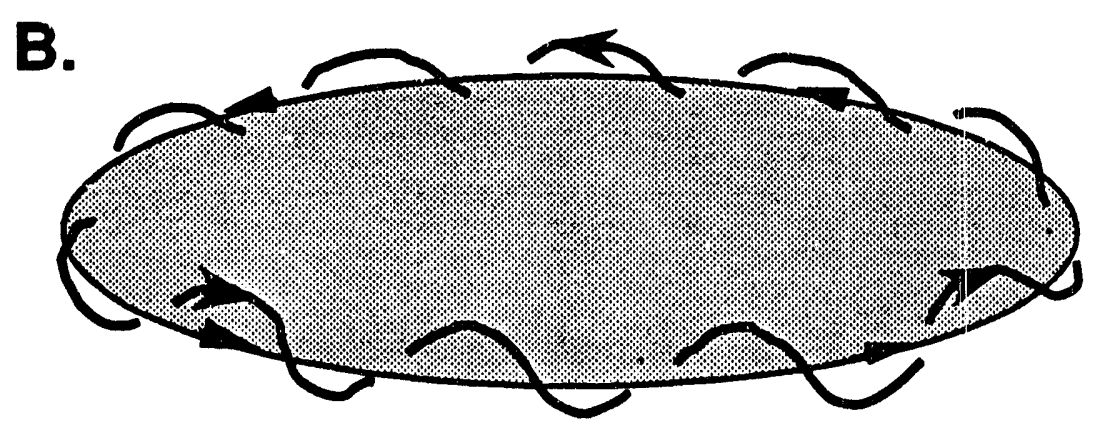

$L k=+10$ 
helix axis, and the result is that right-handed DNA will necessarily have a positive linking number.

As a topological property, $L k$ is invariant under any deformation of the helix. This means that $L k$ is not an informative descriptor of the geometry of DNA. The shape of the DNA is better described by two other functions, twist (Tw) and writhe (Wr), which are intimately related to $\mathrm{Lk}$ :

$$
\mathrm{Lk}=\mathrm{Tw}+\mathrm{Wr}
$$

To a first approximation, Wr describes the out of plane or tertiary structure of the DNA, and Tw describes the local geometry of the helix axis. For a given value of $\mathrm{Lk}$, there are infinite geometries, combinations of $\mathrm{Tw}$ and $\mathrm{Wr}$, which can result in the same topology for a DNA molecule.

Supercoiled DNA is usually modeled as being wrapped on a toroidal or spheroidal surface (Fig 1.2) (White et al., 1988). Although $\mathbf{L k}$ is invariant in the transition between these supercoiled forms, the energetics and structure of the DNA, and therefore its biological function, can vary enormously. Consider a circular plasmid wrapped by histones to form a so-called minichromosome (De Bernardin et al., 1986; Sogo et al., 1986). The DNA is constrained by the favorable free energy of nucleosome formation to lie on the surface of a torus (Fig 1.2A). In the presence of a nicking-closing enzyme, the linker DNA between nucleosomes is relaxed. At the surface of the nucleosome, the helical repeat is found experimentally to be approximately $10.1 \mathrm{bp} /$ turn (Drew and Calladine, 1987) compared to the internucleosomal linker DNA which has a helical repeat of 10.5 

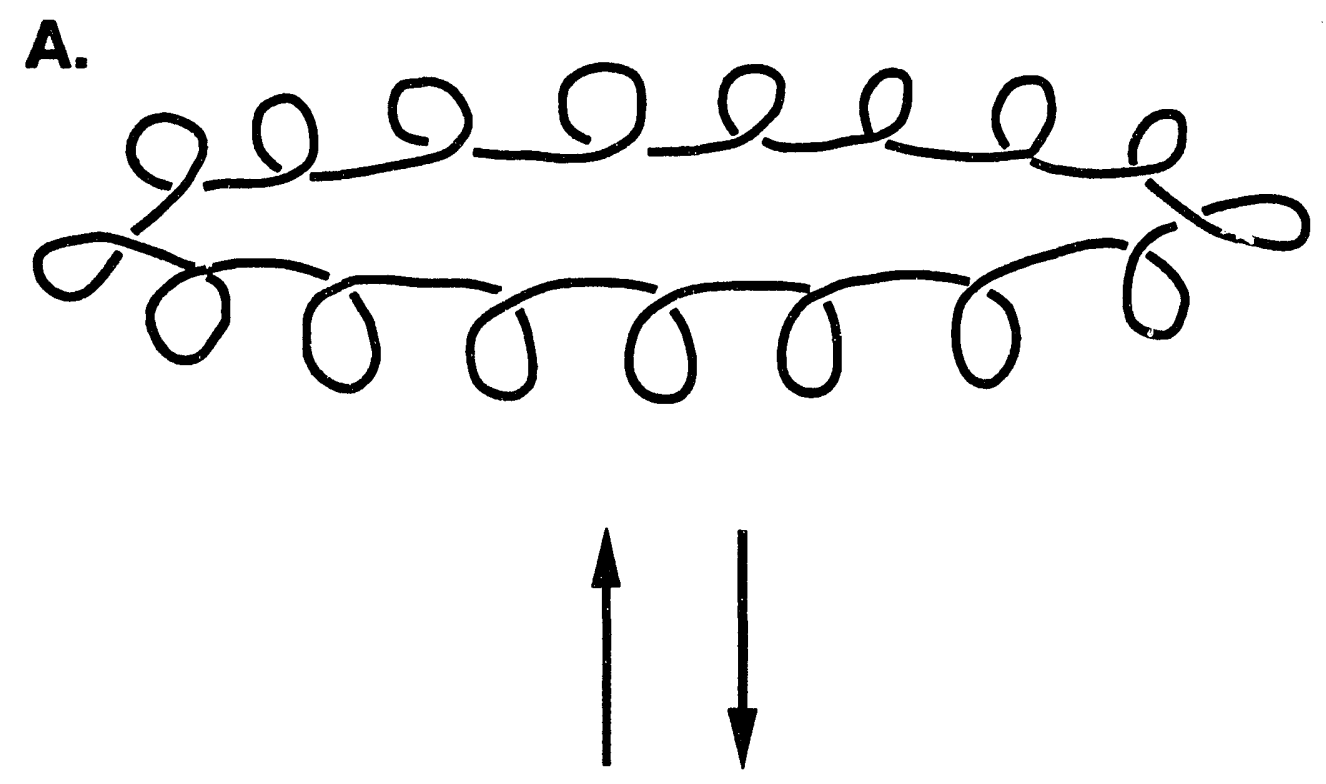

B.

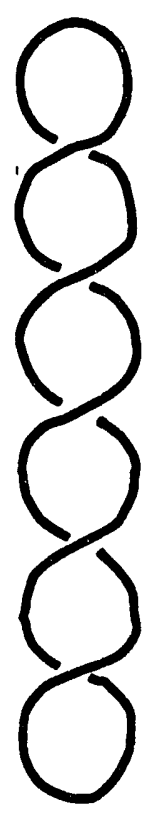

Fig 1.2. Illustration of (A) solenoidal and (B) plectonemically supercoiled DNA. Proteins are omitted from (A) for clarity, although the available evidence suggests that only (B) exists in solution (Boles, 1990; Torbet, 1989). The solenoidal DNA is visualized as lying on a torus while the plectonemic DNA is wrapped on a spheroid. 
bp/turn (Rhodes and Klug, 1980; Shore and Baldwin, 1983b; Wang, 1979). The helical repeat averaged over the whole molecule is therefore somewhat smaller than $10.5 \mathrm{bp} / \mathrm{turn}$. The writhing of the helix axis around the histone octamers resuits in a left-handed wrapping about the axis of a torus, and thus a negative Wr. (In this example, $\mathrm{Wr}$ is a measure of the "tightness" of the wrapping: more ciosely spaced coils create a more negative Wr.)

If all proteins are then removed from the sample, say by phenol extraction, the geometry of the DNA will no longer be constrained by protein interactions. It will assume some new conformation, but the value of $\mathrm{Lk}$ will be unchanged(Fig 1.2B). The DNA structure, once tightly wrapped around nucleosomes, gives way to a more gradually wound structure characteristic of the supercoils of DNA free in solution (Boles et al., 1990; Torbet and DiCapua, 1989). The helical repeat will increase to some new value greater than $10.5 \mathrm{bp} / \mathrm{turn}$, characteristic of underwound DNA in solution. This means that $\mathrm{Tw}$ must decrease and consequently $\mathrm{Wr}$ must increase. The increase in $\mathrm{Wr}$ corresponds, intuitively, to a more loosely wrapped structure in the absence of histones. The geometry of the DNA is vastly different in these two cases, yet $\mathrm{Lk}$ is invariant. In addition, the energy stored in the helix for biochemical processes is different in the two situations. For the minichromosome, the system achieves its lowest energy, since the topoisomerase nicks and reseals the linker DNA until an equilibrium state is achieved. With histones firmly bound, there is no driving force by supercoiling for denaturing the DNA helix. Upon removal of all proteins, the DNA assumes some strained, underwound 
conformation in solution. This strain energy could be used by an RNA polymerase to denature the DNA locally.

This example illustrates the point that $L k$ by itself is not a robust descriptor of the DNA in terms of either geometry or energetics. For comparison between biochemically similar samples, however, measurements of $\mathrm{Lk}$ can be highly informative. In addition, the technique of measuring $\mathrm{Lk}$ (or more properly $\Delta \mathrm{Lk}$ ) is perhaps the simplest method for determining changes in: superhelicity. (The technique for measuring $\Delta \mathrm{Lk}$ is described in detail in Chapter 2.)

A theory, Surface Linking Theory, explicitly relates $\mathrm{Lk}$ to the helical repeat, h, of the helix (White et al., 1988). This theory reformulates $\mathrm{Lk}$ to be the sum of two orthogonal variables which are sensitive functions of the (virtual) surface on which the DNA is wrapped:

$$
\mathrm{Lk}=\mathrm{SLk}+\Phi
$$

In this reformulation, $\Phi$ is the winding number of the DNA and is related to the helical repeat: $\Phi=\mathrm{N} / \mathrm{h}$ where $\mathrm{N}$ is the number of base pairs in the molecule. $\Phi$ is the number of turns in the DNA helix defined relative to a normal vector from the surface on which the DNA lies. $\Phi$ therefore provides information about the average secondary structure in the local reference frame. SLk, the surface linking number, incorporates the tertiary structural components of Tw, denoted surface twist (STw), as well as Wr (White and Bauer, 1988):

$$
S L k=S T w+W r
$$


SLk is readily determined for many cases of biological interest (White and Bauer, 1988; White et al., 1988). For free DNA in solution, SLk equals 0. For DNA wrapped on a torus, SLk is equal to the number of turns around the torus. SLk and $\Phi$ are invariant for all smooth deformations of the DNA. Smooth deformations of a DNA molecule are those which preserve the spheroid or torus; deformations which alter the surface by introducing breaks or tears, such as a spheroidal to toroidal deformation, are not smooth and thus do not conserve the value of SLk or $\Phi$.

Energetics and structure of supercoiled DNA. Free DNA in solution is usualiy described as an interwound or plectonemic superhelix (Fig 1.2B) (Boles et al., 1990; Torbet and DiCapua, 1989). Current models describing the energetics of supercoiling do not distinguish between positive or negative supercoils. At the level of secondary structure, however, it is clear that there must be important differences between overwound and underwound DNA. Negatively supercoiled DNA is known to undergo a variety of structural transitions which often depend on specific sequences. Positively supercoiled DNA should be unable to exhibit these same alterations since, in general, they depend on the ability of the DNA to unwind locally, a highly unfavorable transition for overwound DNA. The focus of this section, therefore, is to lay out the general principles for the structures and energetics of DNA supercoiling, while at the same time pointing out some of the unresolved issues.

Traditionally, references in the literature to positively supercoiled DNA refer to covalently closed, circular molecules in the 
presence of high concentrations of an intercalator (c.f. Bauer, 1968; Worcel, 1972; and Wang, 1971). Under these conditions, a covalently closed DNA is considered to be overwound relative to a molecule which is nicked in the presence of intercalator and then resealed by a ligase. The binding of intercalator unwinds the nicked DNA; resealing creates a covalently closed species at a new equilibrium linking number $\mathrm{Lk}_{\mathrm{o}}{ }^{-}$which is smaller than $\mathrm{Lk}_{\mathrm{o}}$ in the absence of intercalator. Recently, however, it has become possible to generate positively supercoiled DNA, not by altering $\mathrm{Lk}_{0}$, but by overwinding plasmid DNA in the absence of intercalator. Two methods can yield preparations of overwound DNA. An enzyme called reverse gyrase has been discovered in Sulfolobus, an archaebacterial thermophile which inhabits highly acidic environments (Kikuchi and Asai, 1984). This enzyme catalyzes the overwinding of DNA with the hydrolysis of ATP. A second method for generating positive supercoils involves the inhibition of the enzyme DNA gyrase in vivo (Lockshon and Morris, 1983). In the absence of gyrase, DNA tracking processes such as replication and transcription can introduce positive superturns into DNA substrates in vivo. It is becoming increasingly clear that overwound DNA may play an important roles in cells (Liu and Wang, 1987; Wu et al., 1988). The availability of positively supercoiled DNA now makes a more thorough understanding in terms of structure and energetics possible.

The energetics of supercoiling were first analyzed by investigating the hydrodynamics of DNA with intercalated ethidium bromide (Bauer and Vinograd, 1970) and later using nicking-closing 
enzymes which could interconvert topoisomers (Depew and Wang, 1975; Pulleyblank et al., 1975). Extensive incubation of covalently closed supercoiled plasmids with a nicking-closing enzyme was shown to give rise to a distribution of topoisomers which could be resolved by agarose gel electrophoresis. This distribution was shown to be an equilibrium mixture of topoisomers by two procedures: (1) isolation of any single topoisomer in the set and reincubation with a nicking-closing enzyme gave rise to a distribution which was indistinguishable from the original; and (2) under the same solution conditions, treatruent of a nicked circular plasmid with DNA ligase was shown to produce an identical distribution of topoisomers. The weight distribution of topoisomers is Gaussian (Depew and Wang, 1975; Pulleyblank et al., 1975).

These observations are consistent with a model in which the free energy of superhelix formation is proportional to the square of the linking difference:

$$
\Delta \mathrm{G}_{\mathrm{sc}}=\mathrm{K}\left(\mathrm{Lk}-\mathrm{Lk}_{\mathrm{o}}\right)^{2}
$$

where $L k$ is the linking number of the topoisomer of interest and $\mathrm{Lk}_{\mathrm{o}}$ is the linking number of the ground state topoisomer. The importance of these results is that they established that the DNA superhelix can store energy which can do useful work in biological processes. In fact, current models for transcription (McClure, 1985), site-specific recombination (Nash, 1990), replication (Bramhill and Kornberg, 1988), and chromosome packing (Olins and Olins, 1974) all invoke the essential importance of the potential energy of supercoiled DNA. 
The linear dependence of $\Delta \mathrm{G}_{\mathrm{sc}}$ on $\Delta \mathrm{Lk}^{2}$ implies that the DNA molecule behaves like an ideal torsional spring over a small range of twisting and bending deformations. But the range of superhelix densities which are accessible in the above experiments is very limited. The linking number distributions are tightly clustered around $L k_{0}$. The free energy function far away from $L k_{0}$ can be measured only using other techniques. The experiments described in Chapter 2 measure the torsion constant $\mathrm{C}$ as a function of superhelicity using the technique of time-resolved fluorescence polarization anisotropy. $\mathrm{C}$ is the coefficient which determines the free energy $\delta g_{t w}$ required to alter the twist angle between base pairs by an amount $\delta \mathrm{Tw}$ :

$$
\delta g_{\mathrm{tw}}=\mathrm{C}(\delta \mathrm{Tw})^{2}
$$

The results presented in Chapter 2 demonstrate that $C$ is a function of the superhelicity of DNA. Thus, over a large range of superhelix densities, the ideal spring analogy is inaccurate.

The torsional and flexural stress of DNA supercoiling can be relieved in a variety of ways. For underwound DNA, the most obvious structural transition is the melting of base pairs in some small region of a much larger molecule. Melting of DNA is energetically costly, at the expense of about $2 \mathrm{kcal} / \mathrm{mol}$ for each denatured base pair (Breslauer et al., 1986), and so does not occur spontaneously to any appreciable extent for physiological superhelix densities. But many biologically relevant processes involve the binding of proteins to denatured regions, which compensates for the unfavorable energetics of strand separation. The single-stranded DNA binding protein of bacteriophage $T 4$, for instance, can shift the 
equilibrium from native duplex to separated strands for linear DNA, because it cooperatively binds to denatured regions (Chase and Williams, 1986). The kinetics of local DNA melting can also be enhanced by negative supercoiling. The rate at which RNA polymerase isomerizes from the closed to the open complex for some promoters is a function of the negative superhelicity of the template (Mc(Elure, 1985).

Negative supercoiling also facilitates other structural transitions of the helix. The formation of Z-DNA (Jaworski et al., 1987'; Rich et al., 1984), H-DNA (Htun and Dahlberg, 1989;

Lyamichev et al., 1985; Volishin et al., 1988), and cruciform structures (Mizuuchi et al., 1982; Sinden et al., 1983) has been shown to depend on DNA underwinding. Conceptually, each of these changes can be thought of as requiring a local denaturation of the DNA helix and thus a release of the stress of negative supercoiling. It has been postulated that these alternate structures may play important regulatory roles in cells and that intracellular supercoiling is important for this reason, among others (Freund et al., 1989; Horwitz and Loeb, 1988; Jaworski et al., 1987).

DNA supercoiling also affects the secondary and tertiary structure of DNA sequences which cannot undergo transitions to non-B-form helices. Some of the superhelical stress is distributed over a change in the three dimensional conformation of the molecule. Statistical mechanical simulations of supercoiled DNA, starting from the experimentally determined bending and torsion constants of DNA, and assuming a harmonic model for the elastic energy of bending and twisting, predict that $\Delta \mathrm{Lk}$ will be partitioned 
about $70 \%$ into $\mathrm{Wr}$ and $30 \%$ into $\Delta \mathrm{Tw}$ (A. V. Vologodskii, personal communication). Experimental measurements using electron microscopy and a recombination assay also give a similar result (Boles et al., 1990).

In addition to changing the helical repeat, supercoiling may have important effects on the elastic properties of DNA as well. To elucidate fully the biological implications of supercoiling, these elastic properties must be understood. For example, negative supercoiling has been show to increase significantly the strength of lac repressor-DNA binding (Borowiec and Gralla, 1987; Wang, 1974; Wang et al., 1974). Positive supercoiling, however, decreases the strength of lac repressor-DNA binding (Kramer et al., 1988). Two possible hypotheses have been put forward to explain this supercoiling dependence. The first model is that underwound DNA accommodates the natural unwinding of the operator region induced by binding of lac repressor to relaxed DNA (Kim and Kim, 1983). The second model is that unwinding of the helix decreases its torsional and flexural stiffness, thus permitting looped structures to form more easily (Kramer et al., 1988). Kramer et al. (1988) suggest that positively supercoiled DNA "would intuitively appear to be more rigid" than negatively supercoiled DNA. They conjecture that the increased rigidity of positively supercoiled DNA would hinder formation of tight intramolecular loops. Experiments described in Chapter 2 of this thesis demonstrate that positively supercoiled DNA is in fact less rigid to torsional deformation than negatively supercoiled DNA. 
Enzymes which alter DNA supercoiling. Enzymes which change the linking number of DNA are called topoisomerases. Topoisomerases have been found throughout the biological world from phage (Liu et al., 1979) to bacteria (Wang, 1971) to viruses (Bauer et al., ) to mammalian cells (Champoux and Dulbecco, 1972). The need for these enzymes was recognized shortly after the discoveries of circular DNA molecules (Cairns, 1963). The separation of intertwined strands during replication as well as the resolution of catenated daughter molecules were particularly troubling topological aspects of semiconservative replication of the double helix. In addition, the known rate of replication made the idea of a swivel attractive even for linear DNA molecules.

Topoisomerases fall into one of two classes, type I or type II, depending on whether they change the linking number of DNA in successive steps of \pm 1 or \pm 2 (Liu and Liu, 1980). All organisms seem to have both type I and type II topoisomerases, but in yeast and eubacteria, only the type II enzymes are absolutely essential, probably due to their requirement in the resolution of the catenated products of DNA replication (DiNardo et al., 1984; Steck and Drlica, 1984). In $E$. coli, deletion mutants wholly lacking the topA gene for topoisomerase I have been constructed (Sternglanz et al., 1981). Genetic and biochemical analysis shows that these strains are viable, primarily because they accumulate compensatory mutations, most of which can be mapped to one of the genes for the subunits of DNA gyrase (DiNardo et al., 1982; Pruss et al., 1982). In yeast, top 1strains are completely viable, and grow at an almost normal rate when compared to isogenic parent strains (Goto and Wang, 1985; 
Thrash et al., 1985). In this case, it is thought that topoisomerase II, because of its similar enzymatic properties, can substitute for the essential functions of the topoisomerase I enzyme.

Mechanistically, all topoisomerase reactions go through a protein-bound DNA intermediate (reviewed in Maxwell and Gellert, 1986). In those cases which have been studied in detail, the covalent attachment is from a tyrosine on the enzyme to a 3'- or 5'phosphate moiety on the DNA backbone. This mechanism conserves the number of phosphoester bonds in the reaction and thus explains the lack of requirement for ATP in many of the relaxation reactions catalyzed by topoisomerase I enzymes. At the structural level, the type II topoisomerases appear to be particularly conserved (Lynn et al., 1986). Their mechanisms show important similarities, including the hydrolysis of ATP and a $5^{\circ}$-phosphate linkage to a tyrosine in the enzyme-DNA complex (Maxwell and Gellert, 1986). Among type I topoisomerases, there is little similarity at either the structural or mechanistic levels, particularly when comparing prokaryotic enzymes to those from eukaryotes (Wang, 1987).

The categorization of topoisomerases into two types, although useful in some respects, overlooks important differences between enzymes (Gellert, 1981; Wang, 1985). Several examples should make clear the diversity of functions within a given class. DNA gyrase, a eubacterial type II topoisomerase, catalyzes the formation of negative supercoils in all DNA substrates, with the concomitant hydrolysis of ATP. Eukaryotic topoisomerase IIs, which also hydrolyzes ATP, can only relax supercoils. Among the type I enzymes, topoisomerase I from $E$. coli, originally known as the $\omega$ 
protein, can work on negatively supercoiled but not positively supercoiled DNA. Eukaryotic topoisomerase I, on the other hand, can relax both positive and negative substrates, like its type II counterpart from higher cells. The differences between enzy'mes reflect mechanistic dissimilarities which are overlooked in the simple classification scheme. The important point is that a detailed understanding of how each enzyme works is essential for interpreting the results of experiments involving DNA topology in vivo. For this reason, a brief description of the eubacterial topoisomerases (primarily the well-studied enzymes from $E$. coli) is given below in order to understand the experiments detailed in later chapters.

Topoisomerase I from $E$. coli was the first topoisomerase to be isolated in purified form (Wang, 1971). It can relax negatively but not positively supercoiled DNA substrates to their ground state. The rate of relaxation, furthermore, is a function of the negative superhelicity of the substrate. The probable reason that topoisomerase I cannot act on positively supercoiled DNA is that it requires a region of single stranded DNA in order to catalyze the relaxation reaction; the denaturation to single stranded DNA would be promoted by negatively, but not positively, supercoiled DNA (Kirkegaard and Wang, 1985). The inability of eubacterial topoisomerase I to relax positively supercoiled DNA has important functional implications in vivo. This fact has been exploited to demonstrate that transcription and other DNA tracking processes can introduce positive and negative supercoils into a DNA molecule in both bacteria and yeast (Giaever and Wang, 1988; Wu et al., 
1988). If this process proves to be of wide importance in bacterial cells, it demonstrates that one of the essential roles of topoisomerase $I$ is to relieve negative domains of supercoiling generated by transcription and replication (Liu and Wang, 1987). Consistent with this hypothesis, it has been shown that topA strains of $E$. coli have higher than normal levels of supercoiling of the plasmid pBR322 and that this negative supercoiling depends on transcription of the tetr gene (Pruss et al., 1982).

The type II topoisomerase in eubacteria, DNA gyrase, is the only enzyme known to increase catalytically the negative superhelicity of DNA substrates (Gellert, 1981; Wang, 1985). Gyrase hydrolyzes ATP in order to drive the reaction uphill in free energy. In the absence of ATP, gyrase can relax supercoiled DNA (Gellert et al., 1977). Another physiologically important function of gyrase is the separation of catenated DNA molecules (Steck and Drlica, 1984; Steck et al., 1984). Temperature sensitive mutants of gyrase cannot separate daughter chromosomes as judged by fluorescence microscopy of cells grown at the restrictive temperature and sedimentation studies of isolated nucleoids. Unlike topoisomerase I, gyrase has a distinct kinetic preference for relaxed DNA substrates. This bias probably results from both the mechanism of gyrase action and the thermodynamics of DNA supercoiling. Gyrase wraps DNA around its outer surface, inducing a positive node in the helix (Kirkegaard and Wang, 1981; Liu and Wang, 1978). This wrapping should be favored by relaxed or positively supercoiled substrate molecules. In addition, the thermodynamic driving force for 
introduction of negative supercoils, generated by the hydrolysis of ATP, is greatest when the DNA is relaxed or positively supercoiled. An important tool in the study of DNA supercoiling is the use of drugs which inhibit gyrase. These drugs act at two distinct points in the catalytic cycle. The first class of molecules, the coumarins, represented by novobiocin and coumermycin $A_{1}$, inhibit the hydrolysis of ATP (Gellert et al., 1976). The mode of inhibition seems to be a competition for the ATP binding site on gyrase, even though neither of the drugs resembles ATP (Sugino et al., 1978). The second class, represented by oxolinic acid and nalidixic acid, traps a covalent gyrase-DNA intermediate (Gellert et al., 1977; Sugino et al., 1977). Treatment of this intermediate with detergents or alkali results in the scission of DNA at the site of protein attachment. This reaction has been used to study the site preference of gyrase action. The overall conclusion is that there is only a limited preference for specific DNA sequences, other than a requisite TG dinucleotide at the cutting site (Morrison and Cozzarelli, 1979). Both classes of drugs are lethal to cells. Mutations which confer resistance to these drugs have been mapped to the genes for the protein subunits of the enzyme, gyrA and $g y r B$ (Gellert et al., 1977; Gellert et al., 1976). Resistance to the ATPase inhibitors map to the gyrB gene, while those for nalidixic and oxolinic acids map to the gyrA gene. The killing action of the latter drugs is thought to be due to the formation of covalent gyrase-DNA adducts which block replication of the chromosome (Drlica, 1984). 
Supercoiling: effects on replication and recombination. The biological role of DNA supercoiling has been the subject of intensive investigation for more than twenty years. Studies have largely focused on the role of topoisomerases in the essential processes of replication, transcription, and recombination (Drlica, 1984). I will only briefly present evidence for the importance of DNA supercoiling in replication and recombination, where the evidence is probably strongest for a direct function of topoisomerase enzymes. The main part of my discussion will focus on the role of topoisomerases and supercoiling in the regulation of transcription, since that subject is most relevant to the experiments in Chapter 3 of this thesis.

Topology plays an important role at the initiation, elongation and termination stages of DNA replication in bacteria (Drlica, 1984). Initiation in vitro from oriC, the $E$. coli chromosomal origin of replication, requires a negatively supercoiled DNA template or the presence of DNA gyrase (Baker et al., 1986). Elongation of re plication in vivo is rapidly inhibited by shifting temperature sensitive gyrase mutants to the restrictive temperature (Kreuzer and Cozzarelli, 1979). Inhibition of DNA synthesis by treatment of cells with oxolinic acid occurs at far lower concentrations than required to maximally inhibit the enzyme (Snyder and Drlica, 1979). Pulse-labeled DNA in vivo, which represents newly synthesized DNA fragments, is cleaved by oxolinic acid into fragments smaller than those from steady-state labeling experiments (Drlica et al., 1980). These observations are consistent with the notion of a specific subset of gyrase molecules associated with the growing replication 
fork which are unusually sensitive to drug treatment. Movement of the replication fork along the chromosome may overwind DNA ahead of the newly synthesized daughter strands, requiring gyrase to act as a swivel. At the termination stage of replication, gyrase is essential for the decatenation of daughter molecules. Nucleoids from temperature sensitive $E$. coli mutants shifted to the restrictive temperature sediment faster than at the permissive temperature, consistent with the idea that daughter chromosomes are interlinked (Steck et al., 1984). Fluorescence microscopy of whole bacteria stained with a DNA-binding dye reveal that nucleoids in these cells are dumbbell-shaped doublets (Steck and Drlica, 1984). The requirement for DNA gyrase in decatenation is further strengthened by the observation that only type II topoisomerases can decatenate covalently closed, double stranded DNA circles (Liu and Liu, 1980). Topoisomerase I requires nicked substrates in order to perform the decatenation reaction (Tse and Wang, 1980).

The link between DNA supercoiling and recombination has been best studied for the case of site-specific recombination of phage lambda (Nash, 1990). In vitro studies of lambda integration first led to the discovery of a requirement for supercoiled DNA in the reaction (Mizuuchi et al., 1978; Mizuuchi and Nash, 1976). Subsequent biochemical analysis led to the isolation of a protein, DNA gyrase, which could supercoil the phage DNA, thus allowing in vitro recombination to proceed (Gellert et al., 1976). The current model for phage lambda integration proposes that the requirement for supercoiled DNA results from a stereospecific tertiary structure formed by the DNA-protein complex during the integration reaction. 
The phage recombination site, attP, consists of a central core where DNA strand breakage occurs and two flanking arms which contain numerous binding sites for the proteins Int and IHF. Binding of these proteins to their sites occurs regardless of whether the DNA is supercoiled, but in vitro recombination proceeds only when the attP containing DNA is negatively supercoiled (Richet et al., 1986). The requirement for negative supercoiling results from the formation of a complex tertiary structure in which proteins distant in primary sequence interact closely. Formation of this structure is favored by negatively, but not positively, supercoiled DNA since the folding induces positive nodes in the DNA (Griffith and Nash, 1985; Richet et al., 1986).

The protein-DNA complex formed on attP is but one example of a large number of biologically important interactions called specialized nucleo-protein structures (SNUPS) (Echols, 1986). These complicated structures often control the specificity and timing of DNA replication, recombination and transcription, and are utilized by the cell for ensuring precise regulation over DNA transactions. In particular, SNUPS may control the process of development by maintaining and controlling gene expression in different tissue types in eukaryotes. If the insights gained through the study of sitespecific recombination in phage lambda prove to be of general importance, then supercoiling of DNA may be critical for the formation and regulation of these structures (Echols, 1986; Nash, 1990). 
Supercoiling and transcription. Transcription in vivo is profoundly affected by changes in DNA supercoiling. RNA synthesis in temperature-sensiti'e gyrase mutants decreases rapidly upon a shift to the restrictive temperature (Kreuzer and Cozzarelli, 1979; Wahle et al., 1985). Similarly, treatment of bacterial caltures with drugs which inhibit gyrase leads to a decrease in total RNA synthesis as well as a loss in titratable supercoils on the chromosome (Manes et al., 1983; Sanzey, 1979). Most genes, in fact, are activated by negative supercoiling of the template (Gellert, 1981). For a number of cases, this requirement for negatively supercoiled DNA has been demonstrated in vitro (Richardson, 1975; Wang, 1974).

Transcription of some loci, however, increases on relaxed DNA templates, while other genes are insensitive to changes in supercoiling (reviewed in Drlica, 1984). To complicate the picture, at least one gene responds differently to supercoiling in vitro and in vivo: Transcription of tyrT is extremely sensitive to supercoiling in vitro, yet analysis in $E$. coli shows that inhibition of gyrase has little effect on its expression in vivo (Lamond, 1985).

The central question posed by many studies is this: Does DNA supercoiling play a regulatory role in gene expression? Given that supercoiling profoundly affects transcription, it does not necessarily follow that the cell can alter DNA supercoiling to achieve true physiological regulation of transcription. Current opinion seems to be divided between those workers who believe that the bacterial cell tends to maintain a constant level of torsional stress in the chromosome (see for example, Menzel and Gellert, 1983), and those who argue that changes in supercoiling, particularly in response to 
environmental stress, are an important mechanism for gene regulation (see for example, Higgins et al., 1988; Yamamoto and Droffener, 1985). Evidence is presented in Chapter 3 that changes in unrestrained supercoiling do not regulate the transition between aerobic and anaerobic metabolism in the gram-negative, photosynthetic bacterium Rhodobacter capsulatus.

To demonstrate a regulatory role for supercoiling, one must demonstrate that changes in DNA supercoiling accompany changes in gene expression. Measurement of supercoiling of whole chromosomes is tedious (Drlica and Snyder, 1978; Worcel and Burgi, 1972), and these measurements are insensitive to local changes in supercoiling. Investigators have instead resorted to the measurement of linking number changes in small reporter plasmids. The relationship between plasmid and chromosomal supercoiling is not always clear, however, and a case has even been reported in which the chromosome is relaxed but a reporter plasmid is highly negatively supercoiled (Pruss et al., 1982). A convincing demonstration of a regulatory role for DNA supercoiling must also establish a mechanism whereby supercoiling is sensitive to environmental stimuli which alter gene expression. Presumably, this mechanism would involve a system for sensing environmental changes and transducing this signal to modify the activity of topoisomerases in the cell (Yamamoto and Droffner, 1985), but alternative schemes have also been proposed which involve changes in the ionic strength and composition of the cytosol that directly affect enzymes involved in DNA supercoiling (Higgins et al., 1988). 
The best documented example of the regulation of transcription by DNA supercoiling is for the expression of the topoisomerase proteins themselves (Menzel and Gellert, 1983; TseDinh, 1985). Transcription of $g y r A$ and $g y r B$ is decreased by relaxation of the DNA template, and the sequences responsible for this modulation are the promoters for these genes (Menzel and Gellert, 1983; Menzel and Gellert, 1987a; Menzel and Gellert, 1987b). Conversely, transcription of topA increases with negative supercoiling (Tse-Dinh, 1985; Tse-Dinh and Beran, 1988). These observations are consistent with a homeostatic control mechanism of unrestrained torsional tension around an optimal set point in cells. The transcription of topoisomerase genes, and thereby the rate of synthesis of the enzymes, could be continually adjusted to maintain a steady state level of DNA supercoiling (Menzel and Gellert, 1983).

Other experiments reinforce the notion of a set point for unrestrained torsional tension in bacteria. These involve an in vivo perturbation of $\mathrm{Lk}_{0}$, the equilibrium linking number of relaxed DNA. If the homeostasis model is correct, alteration of $\mathrm{Lk}_{0}$ should result in a rapid change in $L k$ to maintain a constant value of $\Delta \mathrm{Lk}$, the steady state underwinding of the DNA. Esposito and Sinden (1987) altered $\mathrm{Lk}_{\mathrm{o}}$ in vivo by treatment of $\mathrm{E}$. coli with chloroquine, an intercalating drug which unwinds DNA. They observed that bacterial cells completely adjusted Lk of reporter plasmids within 5 min after chloroquine treatment and that the changes were in the direction predicted by the homeostasis model. Removal of drug was followed by an equally rapid readjustment of $L k$ back to the initial value. Goldstein and Drlica (1984) performed temperature shifts on wild 
type bacterial cultures as a way to perturb $\mathrm{Lk}_{0}$ in vivo. Although the effects of temperature on bacterial physiology may be complex, these authors observed a relatively rapid linking number changes for two different plasmids which were quantitatively consistent with the known in vitro temperature dependence of $\mathbf{L k}_{0}$.

Several biochemical observations concerning the activity of the topoisomerases enzymes in E. coli further strengthen the homeostasis model. The affinity of gyrase for relaxed DNA (Gellert et al., 1976) and of topoisomerase I for negatively supercoiled substrates (Wang, 1971) occurs at two levels: substrate binding and turnover rate. This could indicate that the topoisomerase activities are titrated over the bacterial chromosome so as to minimize local variations in superhelix density.

The evidence in favor of a regulatory role for DNA supercoiling is based primarily on genetic experiments. The initial observation, by Yamamoto and Droffner (1985), was that topA mutations lead to an inability to grow under aerobic conditions and that gyr mutations result in a deficiency under anaerobic conditions. These authors also claimed to have observed changes in the torsional tension of the bacterial chromosome under aerobic and anaerobic growth conditions in wild type strains, but they did not present their evidence for this statement (Yamamoto and Droffner, 1985). These results were interpreted to mean that, under anaerobic conditions, the bacterial cell increases the negative supercoiling of the chromosome, altering global patterns of transcription and thus activating essential genes for anaerobic metabolism. 
Since the publication of the Yamamoto and Droffner paper, many researchers have used drugs to inhibit DNA gyrase and demonstrate an effect on essential genes for anaerobic metabolism (Dimri and Das, 1988; Dixon et al., 1988; Kranz and Haselkorn, 1986; Novak and Maier, 1987; Zhu and Hearst, 1988). The problem with inhibitor studies is that they lack specificity, since inhibition of gyrase is known to have many pleiotropic effects on cellular physiology. Drug inhibition studies therefore do not demonstrate a true regulatory role for DNA supercoiling.

A more interesting series of papers by Higgins and collaborators make a stronger case for the involvement of supercoiling in response to environmental stress (Bhriain et al., 1989; Dorman et al., 1988; Dorman et al., 1990; Dorman et al., 1989; Graeme-Cook et al., 1989; Higgins et al., 1988; Richardson et al., 1988). Typically, these papers combine genetics, analysis of topoisomer distributions, drug inhibition of gyrase, and changes in environmental conditions to develop their argument. The results from these papers are complicated and sometimes contradictory, but nevertheless suggestive. Taken together, these genetic experiments provide some evidence of a role for DNA topology in response to environmental stresses such as osmotic shock, aerobic to anaerobic shifts, and temperature and $\mathrm{pH}$ variations.

The initial evidence from Higgins and coworkers for regulation by changes in DNA supercoiling comes in the study of the proU locus on the E. coli chromosome (Higgins et al., 1988). proU has been shown to encode three genes, one of which is responsible for the transport of glycine betaine, a zwitterionic quaternary amine which 
acts as an osmoprotectant in bacterial cells (Stirling et al., 1989). Higgins et al. showed that an increase in osmolarity of the growth medium results in an increased linking deficit of reporter plasmids and an increase in proU transcription. Addition of low levels ( $2 \mathrm{mM}$ ) of glycine betaine to the medium reverses this effect (Higgins et al., 1988). topA point mutants increase both plasmid supercoiling and proU expression, while gyrase inhibition has the opposite effects. These results are consistent with a role for negative supercoiling in the activation of transcription of proU. However, deletions of the topA gene, which also increase negative supercoiling of reporter plasmids, drastically decrease in proU transcription. Thus correlation between supercoiling and transcription is not always evident.

The Higgins group also studied the expression of $\operatorname{ton} B$, an aerobically expressed outer membrane protein which is important for iron transport (Dorman et al, 1988). They determined that $\operatorname{ton} B$ is activated in the presence of gyrase inhibitors. Furthermore, they demonstrated that plasmid DNA isolated from aerobic cultures has a larger linking number than that isolated from anaerobic cultures. These findings are consistent with the original Yamamoto-Droffner model. However, ton $B-l a c Z$ fusions are also expressed at fully induced levels under anaerobic conditions when iron-deficiency becomes growth limiting (Dorman et al., 1988). Furthermore, I would argue, their correlation between DNA supercoiling and tonB expression as a function of growth phase is very poor: ton $B$ gene expression changes significantly even when there is no discernable change in DNA supercoiling (c.f. Fig 4, Dorman et al., 1988). 
Dorman et al. attempt to make a general test of the YamamotoDroffner model for a number of anaerobically derepressed lac $Z$ fusions by testing their response to the gyrase inhibitor, novobiocin. These genes map to very different locations on the Salmonella typhimurium chromosome. Three of the fusions were completely unaffected by novobiocin, expression of one fusion was increased, and another was repressed upon treatment with novobiocin.

Dorman et al. conclude that the Yamamoto-Droffner model, although appropriate for tonB expression, is not generalizable to all oxygenregulated genes (Dorman et al., 1988).

Higgins et al. (1988) identified compensatory mutants which could increase expression of the proU locus. They designated this locus osmZ. I believe that these mutants may help to reconcile the apparent contradictions between the homeostasis model and the environmental regulation model for DNA supercoiling in vivo. The $o s m Z$ mutants consistently express proU at higher than wild-type levels. osm $Z$ mutations also have pleiotropic effects on site-specific DNA inversions associated with fimbrial phase variation in Salmonella, expression of the cryptic $b g l$ operon for $\beta$-glucoside metabolism, and the osmotic regulation of genes for the outer membrane porins, OmpC and OmpF. Plasmids isolated from osmZ strains have a decreased linking number compared to DNA from the wild-type. Again, there is an apparent correlation between DNA topology and proU expression.

Significantly, the osmZ locus contains one gene which codes for a small, histone-like protein known as $\mathrm{H} 1$ or HNS (Dorman et al., 1990). I propose that increases levels of protein $\mathrm{H} 1$ in $o s m Z$ strains 
could be responsible for changes in both DNA linking number and proU transcription. Binding of $\mathrm{H} 1$ would form left-handed (negative) superturns in the DNA (Broyles and Pettijohn, 1986) and thereby induce compensating right-handed (positive) supercoils elsewhere. Removal of the positive supercoils by gyrase would decrease the linking number of the DNA, while at the same time maintaining the free torsional tension at a constant level. Upon analysis in a gel, in the absence of $\mathrm{Hl}$, the DNA would appear to be more negatively supercoiled.

The correspondence between $o s m Z$ and a histone-like protein is, in fact, intriguing. It is becoming quite clear that histone-like proteins function in bacteria to increase the flexibility and thus affect the conformation of DNA (Flashner and Gralla, 1988; HodgesGarcia et al., 1989; Pettijohn, 1988; Johnson et al., 1986). An increase in $\mathrm{H} 1$ expression in the osmZ strains should increase DNA bending flexibility. Osmotic shock could also increase DNA bending flexibility, either directly by shielding phosphate interactions on the DNA backbone or indirectly by inducing expression of histone-like proteins. The correspondence between osm $Z$ mutations and osmotic shock on proU expression may therefore be reconciled in terms of a model which underscores the importance of DNA flexibility. Induction of $\mathrm{H} 1$ protein or an increase in the ionic strength of the cytosol may both activate transcription of proU by enhancing DNA flexibility, which allows the appropriate structure to form at the promoter. Changes in plasmid linking number may be a secondary effect of the induction of histone-like proteins in response to environmental stress. In this regard, it is interesting that $h u p A B$ 
double mutants in E. coli, which lack the $\alpha$ and $\beta$ subunits of the histone-like protein $\mathrm{HU}$, are unable to cope with either cold or heat shock, a classic environmental stress (Wada et al, 1988).

The role of topoisomerases in the elongation step of transcription. Recently, a new role for topoisomerases in transcription has been proposed, based on the observation that tracking of RNA polymerase along the template can lead to local changes in DNA topology (Liu and Wang, 1987). The suggestion that transcription may require a swivel has long appeared in the literature, but only recently has there been conclusive evidence that RNA polymerase can drive the DNA template (Brill and Sternglanz, 1988; Giaever and Wang, 1988; Tsao et al., 1989; Wu et al., 1988; Rahmouni and Wells, 1989). This "twin domain model" is illustrated in Fig 1.3 (adapted from Liu and Wang, 1987) in which the macromolecular complex (R) is a transcription ensemble consisting of an RNA polymerase, a nascent RNA molecule, and in prokaryotes, a collection of translating ribosomes. Under conditions where the anchoring points (A) are fixed, the transcription ensemble generates domains of positively and negatively supercoiled DNA in front of and behind, respectively, the tracking complex. The rate of change in superhelicity depends on the rate of transcription and on the hydrodynamic frictional drag on $R$. In the absence of topoisomerase proteins to relieve the overwinding or underwinding of the DNA, supercoils could, in the limit, be generated at a rate approaching the movement of the polymerase on the template, namely 3 to 6 turns per second. 


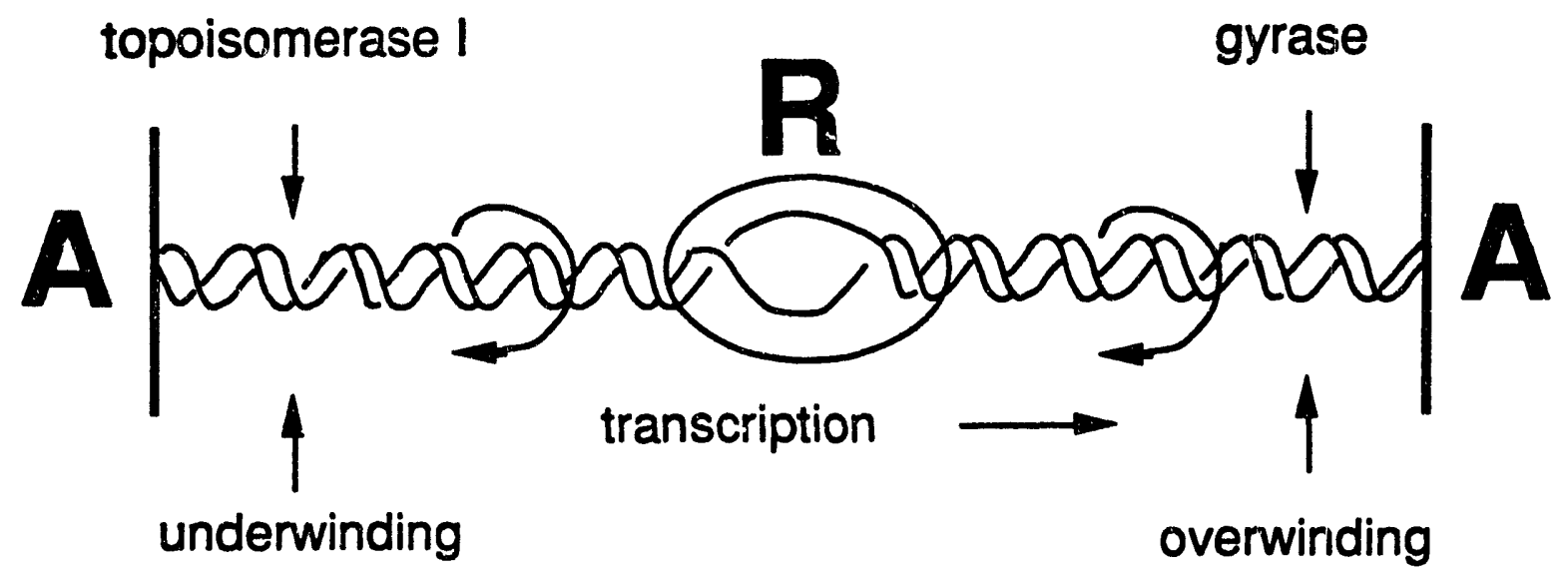

Fig 1.3. The twin domain model for transcription elongation. The transcription ensemble, $R$, consists of RNA polymerase, nascent RNA, ribosomes, and nascent protein. The anchors, $A$, may result from a variety of interactions with the DNA which are described in the text. The domains of supercoiling are shown as are the regions where each of the topoisomerases is expected to act. 
Many factors, such as the number and size of the transcription ensembles $R$, the stability of the anchors $A$, the viscosity of the medium, and the activity of topoisomerases will determine the efficiency of generating supercoiled domains in vivo. Currently, the importance of these variables is not known. The requirement that the anchors $A$ are fixed and that the frictional drag on $R$ be large may be achieved in a variety of situations (Liu and Wang, 1987). For instance, if points $A$ represent attachment sites to a membrane (Ogden et al., 1988) or a nuclear scaffold (Paulson and Laemmli, 1977), then they may be for practical purposes fixed. Similarly, if coupled transcription-translation at $R$ generates a nascent protein which is being inserted into a membrane, then $R$ may have an effectively large drag. Such an insertional mechanism has been postulated for the tet gene on plasmid pBR322 (Lodge et al., 1989). Finally, the anchors A may themselves be oppositely oriented transcription complexes on a circular template which in effect generates two domains within the molecule (Wu et al., 1988).

The twin domain model is a further extension of the notion that topoisomerases strive to mairtain some average superhelical tension in cells. If topoisomerases are preferentially associated with heavily transcribed regions of the bacterial genome, as already appears to be the case in eukaryotic cells (Fleischmann et al., 1984; Gilmour et al., 1986), then this distribution could help to relieve the torsional stress induced by transcription. Inhibition of DNA gyrase might, under these conditions, inhibit elongation by allowing the formation of positive supercoils in the DNA template. Thus, some of the observed effects of gyrase inhibitors in cells might be due to the 
requirement for a swivel and not the activation of the template by negative supercoiling.

The twin domain model also offers the possibility for reconciling some of the conflicting observations about the effects of topoisomerase mutations in vivo. Loss of topoisomerase function might have different effects depending on whether a gene is plasmid encoded or found on the chromosome. The context of a gene and the activity of nearby genes may have important consequences for expression (Pruss and Drlica, 1989).

Outline of the thesis. Each of the following chapters describes an experiment which relates in some way to DNA supercoiling. The experiments in Chapter 2 measure the torsion elastic constant of DNA in vitro as a function of superhelix density using the technique of time-resolved fluorescence polarization anisotropy. The results from these experiments establish that the resistance of DNA to twisting varies as a function of both supercoiling and ionic strength. They further suggest that the classical thermodynamic picture of DNA supercoiling as being analogous to the winding of an ideal torsional spring may require some revision.

In Chapter 3, experiments are presented to test the model that a stable change in the free torsional tension of DNA in vivo regulates the expression of genes for anaerobic metabolism in gram-negative bacteria. The results suggest that there is no stable change in unrestrained supercoiling within the photosynthesis gene cluster of Rhodobacter capsulatus, a purple, non-sulfur photosynthetic bacterium upon induction of anaerobic metabolism. Additional 
experiments are described which indicate that transient changes in DNA supercoiling may occur on the chromosome. These results are interpreted in terms of the twin domain model for transcriptional supercoiling.

Finally, the experiments in Chapter 4 outline an attempt to develop a model system for studying transcriptional supercoiling. The aim of these experiments is to understand the physical requirements and the biological consequences of transcriptional supercoiling. 
For number sequence only 


\section{Chapter 2 \\ Time-resolved Fluorescence Polarization Anisotropy of Superhelical DNAs}

The ability of DNA to bend and twist is critical to its function in the storage and expression of genetic information. The flexibility of DNA can vary as a function of sequence (Gartenberg and Crothers, 1988), solvent conditions, and protein binding (Hodges-Garcia et al., 1989), among other variables. Proteins often exploit the flexibility of DNA by folding together distant sequences of the elongated polymer into a compact, three-dimensional structure in order to achieve precise biological regulation (Echols, 1986). Alignment of DNA sequence elements with protein binding sites can require both bending and twisting of the helix. In this chapter, experiments are described which measure the torsional stiffness of DNA as a function of superhelix density using the technique of time-resolved fluorescence polarization anisotropy. The sensitivity of the method is demonstrated by measuring the twisting flexibility of lambda DNA in solutions containing sucrose. The dynamics of lambda DNA vary as a function of viscosity, just as predicted by the theory which describes DNA twisting. Torsional stiffness is shown to be inversely related to ionic strength, consistent with an important role for ionic interactions in determining the twisting flexibility of the DNA. At physiological salt concentrations, the torsion constant of DNA varies monotonically with increasing negative superhelix density over the range of $\sigma=+0.042$ to -0.123 . Furthermore, at low ionic strength ( $\mu$ 
$=5 \mathrm{mM})$, the torsional stiffness of topoisomers undergoes a sharp transition between positively and negatively supercoiled samples.

\section{Introduction}

A striking example of the importance of DNA flexibility has been elaborated in studies of the mechanism of genetic regulation by certain phage and bacterial DNA binding proteins (Dunn et al., 1984; Hochschild and Ptashne, 1986; Majumdar and Adhya, 1984). A common motif involves a regulatory protein, usually a repressor, that binds to two different sites on DNA that are separated by a distance larger than the dimensions of the protein itself (Adhya, 1989). The protein may be bidentate, with two distinct DNA binding sites on the same molecule, as is the case for the lac repressor; or two monomers can interact cooperatively through protein-protein contacts to enhance the overall stability of binding, as in the case of the $\lambda$ repressor. A variety of experiments have demonstrated that the DNA is looped out between binding sites to form a smooth bend upon complex formation (Griffith et al., 1986; Hochschild and Ptashne, 1986; Kramer et al., 1987). The strength of the interaction has furthermore been shown to depend periodically on the number of base pairs in the loop. Changing the number of base pairs can dramatically alter the stability of the complex. The period between binding maxima is 10 to 11 base pairs, or one turn of the helix. Optimal interaction results when the DNA sites are located on the same face of the helix. Displacement by one-half turn decreases the strength of the interaction to a minimum. These observations demonstrate that the torsional stiffness of DNA can have important 
implications for biological function. The favorable free energy of protein binding to DNA must compensate for the flexural strain imposed by the looping of the DNA. An additional requirement for DNA twisting to bring the protein binding sites into angular alignment can significantly shift the binding equilibrium. This torsional strain is minimized when binding sites are separated by an integral number of turns of the DNA helix.

Sequence-specific variations in bending and twisting flexibility may also play a role in biological function. At the lac operon, specific sequences increase the bending flexibility in the promoter region, contributing to correct regulation of transcription (Gartenberg and Crothers, 1988; Liu-Johnson et al., 1986). Binding of the cyclic AMP repressor protein (CRP) to its site near the lac promoter induces a bend in the DNA which is critical to the activation of lac expression. The ability of CRP to deform the DNA has been shown to depend on specific base pairs which are not contacted by the protein (Gartenberg and Crothers, 1988). These base pairs allow the DNA to bend more easily and thus facilitate the interaction with CRP.

The twisting flexibility of specific sequences has also been proposed to facilitate the binding of a protein to its target site (Koudelka et al., 1988; Koudelka et al., 1987). Crystal structure studies of phage 434 repressor bound to its palindromic operator demonstrate that the central base pairs, with which 434 repressor does not make contact, are overwound relative to free DNA (Aggarwal et al., 1988; Wolberger et al., 1988). Mutagenesis studies show that changing the central A.T base pair to G.C reduces the 
binding affinity of 434 repressor by approximately fifty-fold (Koudelka et al., 1988; Koudelka et al., 1987). The authors of this work speculate that the change in binding constant may reflect a difference in the torsional stiffness of the central base pairs which aiters the ability of the protein to deform the helix.

To measure the stiffness of DNA, either static or dynamic properties can be investigated. For example, the persistence length is a static measure of the bending stiffness of DNA, and it can be measured by a variety of hydrodynamic techniques (Cantor and Schimmel, 1980). The bending stiffness also contributes to dynamic properties of DNA in solution. The lifetimes of the bending normal modes span a wide time scale, from $10^{-9}$ to $10^{-5} \mathrm{sec}$ or longer (Fig 2.1) (Barkley and Zimm, 1979; Scalettar et al., 1990). The bending stiffness will affect the amplitudes of these normal modes, and thus the overall dynamics of bending. Unfortunately, there is no theory which relates time-dependent flexural reorientation to a measurable spectroscopic property. Therefore, the best means of measuring bending stifffness are the static, hydrodynamic techniques.

To determine the torsional stiffness of DNA, both static and dynamic techniques have been used. The static method measures the probability of cyclization of short DNAs. less than 500 base pairs, as a function of the number of base pairs (Shore and Baldwin, 1983a; Shore and Baldwin, 1983b). Cyclization probability is measured by determining the ratio of rates for cyclization vs. oligomerization at the early times in a ligation reaction. For small molecules, where the helix is not expected to writhe, the cyclization probability varies periodically as a function of the DNA length. The 
Fig 2.1 Some characteristic times for probing DNA dynamics

Electric Birefringence

EPR

Singlet State Depletion: Fluorescence (pFRAP)

Singlet State Depletion: Absorption

Fluorescence Depolarization
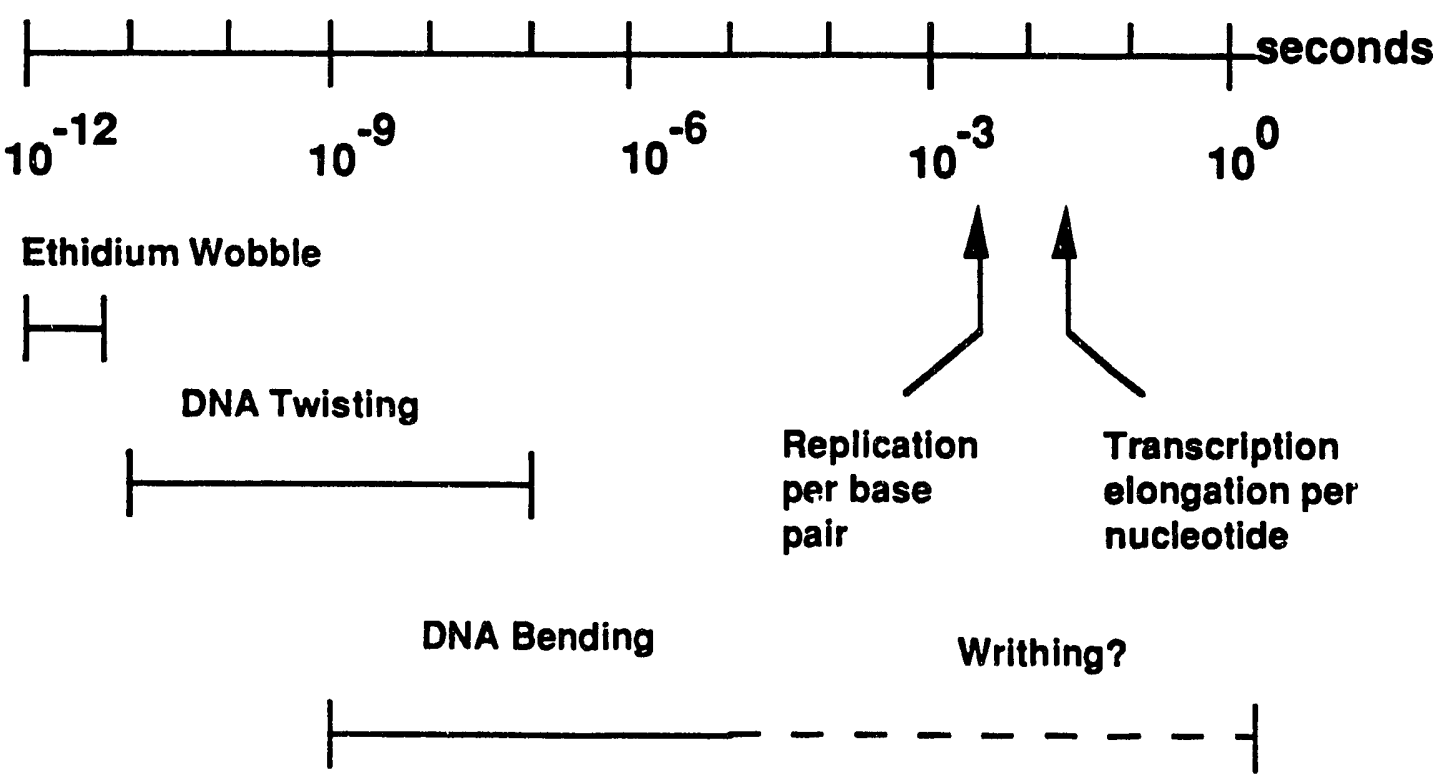

Imino proton exchange

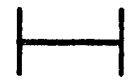

psec nsec $\quad \mu$ sec msec second 
period is equal to $10.45 \mathrm{bp}$, or exactly the helical repeat of DNA (Shore and Baldwin, 1983a). The difference in cyclization probability between molecules which differ by only a few base pairs in length is a measure of the free energy required to introduce a fraction of a turn into the DNA and thus bring the ends into angular alignment. Conceptually, this cyclization experiment is similar to the looping of DNA by regulatory proteins. Careful measurement of cyclization probabilities has provided good estimates of the torsional stiffness of relaxed DNA (Shore and Baldwin, 1983a; Shore and Baldwin, 1983b; Taylor and Hagerman, 1990).

Dynamic measurements of torsional stiffness use timeresolved spectroscopic techniques, in particular time-resolved fluorescence anisotropy of intercalated ethidium bromide (Fujimoto and Schurr, 1990; Millar et al., 1982; Thomas and Schurr, 1983; Wu et al., 1988). Ethidium binds tightly to the helix, with an off-rate of approximately $1 \mathrm{msec}^{-1}$, and its fluorescence dipole is approximately perpendicular to the long DNA axis (Bresloff and Crothers, 1975; Hogan et al., 1979). Collisions of the DNA with solvent result in Brownian twisting oscillations which tend to reorient the fluorescence dipole. The rate at which this reorientation occurs is a measure of the torsional stiffness of the DNA. The advantage of a dynamic measurement is that torsional stiffness can be investigated under a variety of conditions in which ligase, the enzyme used for cyclization studies, does not function. Thus, torsional stiffness has been investigated as a function of temperature up to $70^{\circ} \mathrm{C}$ (Thomas and Schurr, 1983), at very low ionic strengths (Shibata et al., 1984), and at a ratio of intercalated chloroquine up to 0.5 per base pair 
(Wu et al., 1988). In this chapter, experiments are described to measure torsional stiffness as a function of superhelicity, an important parameter of DNA structure which cannot be addressed using static methods.

To observe molecular dynamics, techniques must be chosen which are compatible with the time scale of the process under investigation (Fig 2.1). DNA twisting occurs on the fastest time scale--between $10^{-10}$ and $10^{-7} \mathrm{sec}-$ of any dynamic mode which is unique to the double helix. This time range correlates well with the lifetime of many commonly used fluorophores, including intercalated ethidium bromide $(\tau=23 \mathrm{nsec})$, and thus fluorescence is a particularly attractive technique for observations of twisting. An additional advantage of fluorescence is its extremely high sensitivity compared to other optical or magnetic techniques. Short wavelength, high frequency twisting modes correspond to the motions of individual base pairs, whereas longer wavelength twisting modes result from the correlated motion of many hundreds of base pairs (Allison and Schurr, 1979; Barkley and Zimm, 1979). Each twisting mode has a characteristic amplitude, which determines the overall decay function of the fluorescence polarization.

All techniques for measuring macromolecular dynamics involve creating a non-equilibrium distribution of molecules and observing the thermal relaxation of this population back to its random equilibrium state (Cantor and Schimmel, 1980). For example, in the technique of flow dichroism, a hydrodynamic shear is applied to a solution of macromolecules by moving two nearby surfaces past one another. The long axis of the macromolecules will 
tend to orient in the direction of flow. The degree to which the sample is aligned will depend on the magnitude of the applied force and the dimensions of the molecule. If the shearing forct is suddenly turned off, the molecules will reorient through random Brownian motion back to an isotropic equilibrium state. For a rodlike molecule such as DNA, the rate of reorientation is a sensitive function of the length of the DNA fragment. Small molecules will tand to reorient quickly while long molecules will rotate more slowly. The rate of reorientation can be monitored with polarized light since DNA preferentially absorbs light polarized perpendicular to the helix axis. Many other techniques, such as electric birefringence, electric dichroism, polarized fluorescence recovery after photobleaching, and electron paramagnetic resonance can be used to monitor various time domains of DNA dynamics (Fig 2.1). In fluorescence polarization anisotropy (FPA), the nonequilibrium distribution of fluorescent molecules is created by illuminating a collection of fluorophores with plane polarized light. The excitation preferentially photoselects those molecules whose absorption dipole is nearly aligned with the polarization of the incoming light according to a $\cos ^{2} \theta$ dependence, where $\theta$ is the angle between the polarization axis and the absorption dipole moment. The subsequent emission of light is therefore polarized. The anisotropic fluorescence distribution will decay with time due to several competing processes, including rotational diffusion. (Other mechanisms of depolarization, such as energy transfer, light scattering, or differences between the angle of absorption and emission dipole moments, can also contribute to depolarization. 
These additional factors can be eliminated by careful choice of experimental conditions.)

The observed decay in fluorescence either perpendicular or parallel to the polarization axis is a convolution of the intrinsic lifetime and the rotational motion of the fluorophore. Fluorescence intensity measured parallel to the excitation axis decays by two mechanisms: Emission by the excited state decreases the total number of fluorophores; and rotation out of the plane of excitation also depletes the population of molecules aligned parallel to the original polarization. Fluorescence intensity measured in the perpendicular mode also decays due to the intrinsic fluorescence lifetime, but it increases due to rotation of excited state molecules into alignment with the perpendicular axis. The anisotropy function, $r(t)$, deconvolutes the intrinsic lifetime from the motions of the fluorophore to yield information about the average angular displacement of the sample as a function of time, as will be shown below.

Under the proper circumstances, FPA can provide insight into molecular rotational dynamics on a time-scale consistent with the fluorescence lifetime of the molecule under study. Time-resolved FPA data can yield rate constants of fundamental dynamic processes for well-understood systems. Typically, time-resolved FPA measurements provide information over the time-range of $10^{-1}$ to $10^{2}$ nsec. To achieve this extraordinary time resolution, single photon counting methods are utilized. Measurements of FPA for molecules of biological interest has provided insight into dynamic systems as diverse as the diffusion of proteins and lipids in 
membrane systems, the association rates between antigens and antibodies, and the flexibility of protein domains (Lakowicz, 1983). In conjunction with other techniques, FPA can provide insight to the relationship between structure and dynamics. For a flexible macromolecule such as DNA, these measurement can yield information about shape and flexibility (Barkley and Zimm, 1979).

\section{Theory}

Fluorescence anisotropy measurements. The steady-state anisotropy, $r$, is defined as the ratio of excess polarization along one axis to the total fluorescence from the sample:

$$
r=\frac{I_{11}-I_{\perp}}{I_{11}+2 I_{\perp}}
$$

where $I_{\|}$is the fluorescence detected parallel to the exiting light and $I_{\perp}$ is that which is perpendicular (Fig 2.2). For a rigid, immobile sample whose absorption and emission dipoles are parallel (say, a small molecule in a vitrified solution), the steady-state anisotropy achieves its maximal value of $r=0.4$. This upper limit is a consequence of the $\cos ^{2} \theta$ dependence of the probability of absorption, where $\theta$ is the angle between the polarization axis and the electric dipole of the absorbing species, and of the random distribution of the molecule in solution. For a molecule which is free to rotate, the anisotropy will fall in the range $0 \leq \mathrm{r} \leq 0.4$. (Note that the anisotropy can have negative values for the case where 


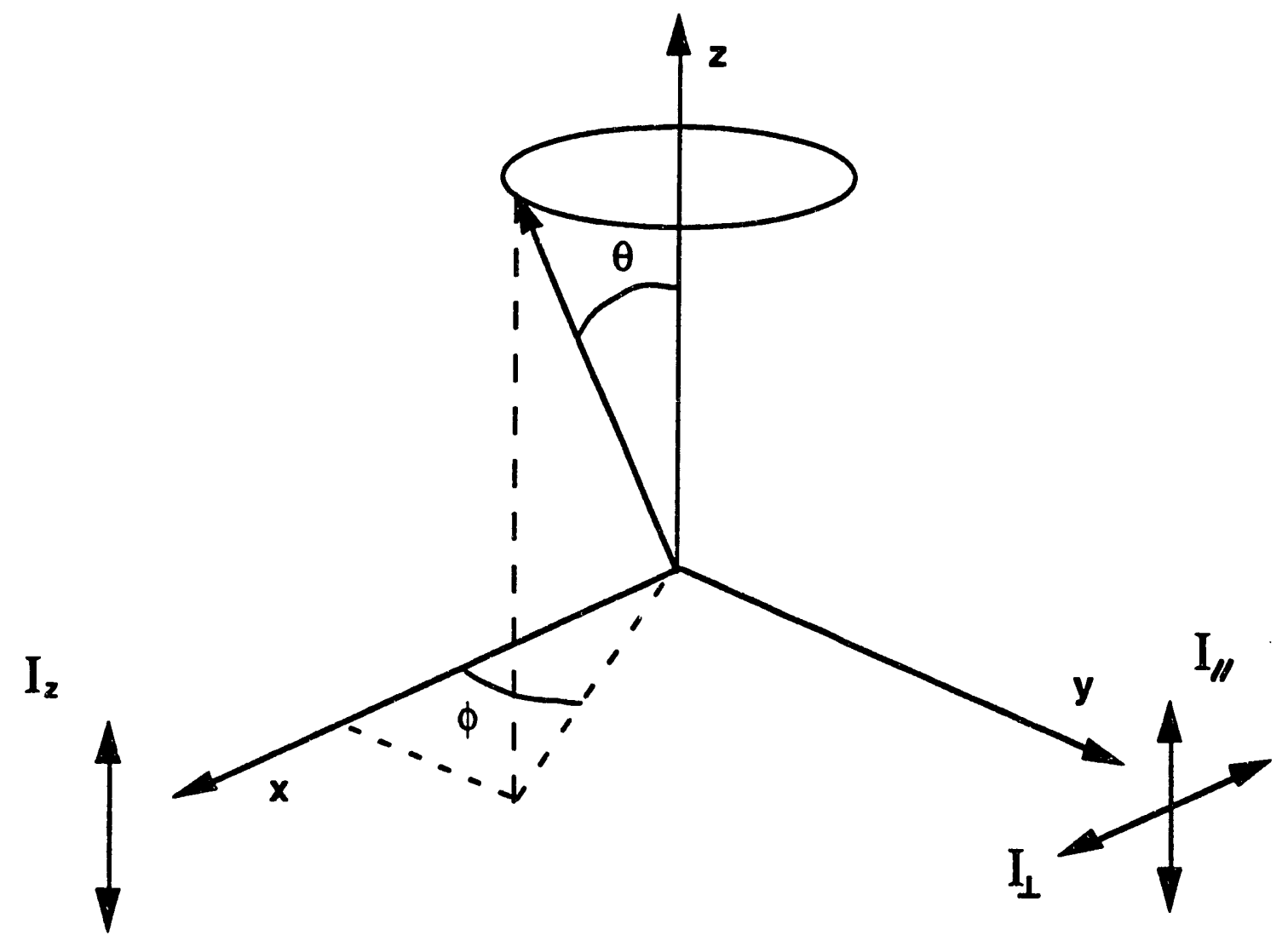

Fig 2.2. The laboratory cartesian coordinate reference frame. Light polarized along the $z$-axis impinges on the sample from the $x$-axis.

A fluorophore, with angle $\theta$ relative to the $z$-axis and angle $\phi$ relative to the $\mathrm{x}$-axis, is excited by the light. Detection along the $\mathrm{y}$-axis resolves the two components, $I_{l l}$ and $I$, of the fluorescence. Steadystate anisotropy measurements detect the average value of $\cos ^{2} \theta$ weighted according to the fluorescence lifetime, while time resolved measurements yield information about the time-dependence of $\cos ^{2} \theta$. 
absorption and emission dipoles are not parallel, a situation which does not occur in these experiments.) Decreasing values of $r$ imply faster rotation on the fluorescence time-scale, and a value of zero would indicate complete randomization of the emission polarization.

The value of the steady-state FPA has a geometrical significance which can be understood by a simple argument (Lakowicz, 1983). Consider the sample under study in the laboratory reference frame where light polarized parallel to the $z$ axis impinges on the sample along the $x$-axis (Fig 2.2). After absorption of a photon and subsequent rotational diffusion, any particular fluorescence dipole will be oriented with angle $\theta$ relative to the $z$-axis; its projection in the xy-plane will make an angle $\phi$ relative to the y-axis (Fig 2.2). Detection along the $y$-axis with an appropriately positioned polarizer will resolve the orthogonal components, $I_{\|}=I_{2}$ and $I_{\perp}=I_{x}$, of the fluorescence. For an arbitrary single fluorescence dipole, with an amplitude $A=1.0$, the detector resolves the components:

$$
\begin{gathered}
I_{z}=\cos ^{2} \theta \\
I_{x}=\sin ^{2} \phi \sin ^{2} \theta
\end{gathered}
$$

For a population of dipoles, the steady-state fluorescence detector sees the average orientation over the lifetime of the fluorophore. Since the distribution of dipoles is always symmetric about the $z$-axis (the excitation axis), all values of $\phi$ from 0 to $2 \pi$ will be equally probable for any given value of $\theta$. We can therefore eliminate the dependence of $I_{x}$ on $\phi$ by taking the average value of $\sin ^{2} \phi:$ 


$$
\overline{\sin ^{2} \phi}=\frac{\int_{0}^{2 \pi} \sin ^{2} \phi d \phi}{\int_{0}^{2 \pi} d \phi}=\frac{1}{2}
$$

Thus, calculation of the steady state anisotropy is a matter of calculating the $\theta$-dependence of $I_{z}$ and $I_{x}$, weighted by the appropriate time-averaged distribution function:

$$
\overline{I_{2}}=\int_{0}^{\frac{\pi}{2}} f(\theta) \cos ^{2} \theta d \theta=\overline{\cos ^{2} \theta}
$$

and

$$
\overline{I_{x}}=\frac{1}{2} \int_{0}^{\frac{\pi}{2}} f(\theta) \sin ^{2} \theta d \theta=\frac{1}{2} \overline{\sin ^{2} \theta}
$$

where $f(\theta)$ is the time-averaged distribution function for the population under study. Using the identity, $\sin ^{2} \theta=1-\cos ^{2} \theta$, one can see that

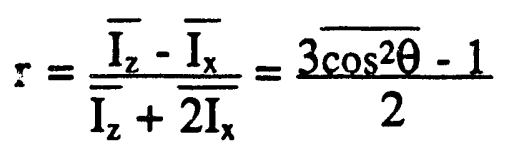

Thus, the steady state anisotropy is a measure of the average $\cos ^{2} \theta$ of the fluorescence dipole weighted over the lifetime of the excited state (Lakowicz, 1983). Complete loss of anisotropy, $r=0$, corresponds to an angle of $\theta=54.7^{\circ}$ which would be the average $\theta$ for a completely random collection of fluorophores in solution.

Time-dependent. FPA measurements yield additional information about the rotation of a fluorophore, since the rate of decay of the anisotropy is a sensitive function of molecular size and shape. For a spherically symmetric molecule, the anisotropy decays 
in an exponential fashion, but for all other cases, the decay is a more complex function. Also, for a large macromolecule such as DNA, FPA is sensitive to hindered rotation where the molecule is not free to rotate in all directions. For high molecular weight DNAs, one does not expect to see large amplitude bending motions or end-over-end tumbling on the fluorescence time scale since these processes have relaxation times in the microsecond and millisecond domains, respectively (Allison and Schurr, 1979; Barkley and Zimm, 1979). Restricted motion in a DNA sample will be evidenced by a leveling off of the anisotropy at a non-zero value at long (i.e. $10^{2} \mathrm{nsec}$ ) times, indicating that the sample has not completely reoriented on the fluorescence time-scale. The time-dependent expression for anisotropy is analogous to that for the steady-state:

$$
r(t)=\frac{I_{11}(t)-I_{\perp}(t)}{I_{11}(t)+2 I_{\perp}(t)}
$$

Similarly, the time-dependent expression is a measure of the change in the average $\cos ^{2} \theta$ as a function of time.

Extracting information from time resolved FPA data requires an adequate model of the system under study, since the fundamental causes of anisotropy decay can be complex. For example, the time-resolved anisotropy of a rigid asymmetric body is a sum of five exponential terms, whose decay depends solely on the principal diffusion constants of the various rotational modes (Barkley and Zimm, 1979; Lakowicz, 1983). For describing DNA torsional motions, there are two competing physical models. The 
first model assumes that DNA can be treated as a uniform, elastic rod which behaves according to classical elasticity and hydrodynamic theories (Barkley and Zimm, 1979). The other model utilizes the bead-spring approximation for DNA in which the individual joints behave as classical torsional springs subject to the hydrodynamic drag of the connected beads (Allison and Schurr, 1979). Although the physical assumptions differ, these models fortunately give rise to identical mathematical descriptions of the torsional motions of the DNA helix over the time range of interest. These models are described in the following sections.

The Barkley-Zimm elastic chain model. In their treatment of the problem of torsional stiffness, Barkley and Zimm (1979) model DNA as a thin, uniformly flexible rod immersed in a viscous fluid at thermal equilibrium. They calculate that the tumbling motion of a rigid, persistence length rod (190 base pairs) about its transverse (short) axis will exhibit a correlation time of about $5 \mathrm{msec}$, too long to contribute significantly to the depolarization of intercalated ethidium bromide in an FPA experiment. They also show that the rotational correlation time of a rigid persistence length fragment about its helical (long) axis is about $100 \mathrm{nsec}$. Furthermore, they argue that the contribution to depolarization by either of these tumbling modes will be even smaller when the segment is part of a high molecular weight DNA (such as that used in the experiments described in this chapter). The Barkley-Zimm analysis, therefore, treats primarily the shorter wavelength torsional and bending modes which relax during the time scale (100 nsec) of an FPA 
experiment. A further simplification is achieved by assuming that angular bending displacements are sufficiently limited on this time scale that they are uncoupled from torsional deformations.

To begin their analysis, Barkley and Zimm derive in normal coordinates the dynamical equations for bending and torsion of an elastic rod for arbitrary initial amplitude of the normal modes using results from classical hydrodynamic and elasticity theories. These general diffusion equations are then adapted to the initial distribution of dipoles in the fluorescence depolarization experiment. The time-dependent anisotropy equation, which incorporates both bending and torsion, can be written as

$$
r(t)=r_{0} \frac{\left[A_{1}(\varepsilon) e^{-\Delta}+A_{2}(\varepsilon)\left[\frac{5}{8}+\frac{3}{8} e^{-\Delta}\right] e^{-\Gamma}+A_{3}(\varepsilon) e^{-\Delta} e^{-\Gamma}\right]}{\left[A_{4}(\varepsilon)+A_{5}(\varepsilon) e^{-\Delta}\right]}
$$

where $r_{0}$ is the limiting anisotropy at time 0 , and $\Gamma$ and $\Delta$ are the time-dependent torsion and bending distribution functions (Barkley and Zimm, 1979; Millar et al., 1982). The $A_{1-5}$ are trigonometric functions of the angle $\varepsilon$ between the ethidium transition dipole and the helix axis:

$$
\begin{gathered}
A_{1}(\varepsilon)=\frac{1}{4}\left(1-3 \cos ^{2} \varepsilon\right) \\
A_{2}(\varepsilon)=\frac{3}{4} \sin ^{4} \varepsilon \\
A_{3}(\varepsilon)=3 \sin ^{2} \varepsilon \cos ^{2} \varepsilon \\
A_{4}(\varepsilon)=\frac{3}{4}\left(1+\cos ^{2} \varepsilon\right) \\
A_{5}(\varepsilon)=\frac{1}{4}\left(1-3 \cos ^{2} \varepsilon\right)
\end{gathered}
$$


The torsion decay function, $\Gamma$, for a rod of length $2 L$ is given by

$$
\Gamma(t)=4 D_{1} t+\left(\frac{4 k_{B} T}{C L}\right) \sum_{k=1}^{\infty} \frac{1-\exp \left(-t / \tau_{k}\right)}{\lambda_{k}^{2}}
$$

which describes the time evolution as a sum of all the torsional decay modes $\mathrm{k}$ with relaxation times $\tau_{\mathrm{k}}$ and wave numbers $\lambda_{\mathrm{k}}=$ $k \pi / \mathrm{L}$. $C$ is the torsional rigidity, $k_{B}$ is Boltzmann's constant, and $T$ is the absolute temperature. The diffusion coefficient $D_{1}$ describes the rotation of the entire rod about its long axis and is important only for long times (generally longer than the observation times of the FPA experiment).

Finally, the torsional relaxation times are shown to be related to the wavelength of the torsional mode and the fundamental parameters of the double helix

$$
\tau_{k}=\frac{4 \pi \eta b^{2}}{C \lambda_{k}^{2}}
$$

where $\eta$ is the solvent viscosity, and $b$ is the helix radius. Barkley and Zimm use this relationship to consider over what times their model might be valid. They argue that as long as the wavelength of an important normal mode is longer than one base pair, then the variations in the local structure of the helix should be small enough to make the elastic rod approximation valid. Taking the wavelength, 
$l_{k}$, of the $k^{\text {th }}$ normal mode to be one base pair, and using the relationship $\lambda_{k}=2 \pi / l_{k}$, they find that torsion modes with wavelengths comparable to the size of a base pair relax with lifetimes of $25 \mathrm{psec}$ or less (Barkley and Zimm, 1979). For longer times typical of the fastest measurements ( $\geq 100 \mathrm{psec}$ ), they argue that FPA should be insensitive to local non-uniformities in the duplex structure of DNA.

A similar analysis for bending (Barkley and Zimm, 1979; Millar et al., 1982) gives

$$
\Delta(t)=4 D_{2} t+\left(\frac{4 k_{B} T}{E I L}\right) \sum_{k=1}^{\infty} \frac{1-\exp \left(-t / \tau_{k}^{\prime}\right)}{\kappa_{k}^{2}}
$$

which describes the superposition of bending normal modes with relaxation times $\tau_{k}^{\prime}$ and wave numbers $\kappa_{k}=(2 k+1) \pi / 4 L$. The leading term corresponds to rotation of the entire rod about its transverse axis with diffusion coefficient $D_{2}$. EI is the bending rigidity of the rod where $\mathrm{E}$ is the Young's modulus and $\mathrm{I}$ is the moment of inertia. The bending relaxation times are

$$
\tau_{\mathrm{k}}^{\prime}=\frac{4 \pi \eta}{\kappa_{\mathrm{k}}^{4} f\left(\kappa_{\mathrm{k}} \mathrm{b}\right)}
$$

where the $f(z)$ is a sum of modified Bessel functions of the form

$$
f(\mathrm{z})=\mathrm{K}_{\mathrm{o}}(\mathrm{z})+\frac{\mathrm{z}}{2} \mathrm{~K}_{1}(\mathrm{z})
$$


An analysis similar to that given above, assuming the shortest bending normal mode to be two turns of the helix (70 $\AA$ ), yields a relaxation time on the order of $100 \mathrm{psec}$. Experimental observations longer than this time should be insensitive to dynamic bending modes which would affect the structural uniformity of the helix and thus compromise the uniform elastic rod assumption (Barkley and Zimm, 1979) .

The complicated expressions for torsion and bending decay can be considerably simplified at certain times in the decay process (Barkley and Zimm, 1979). At short times, the summation in equation [4], which describes the time-dependent angular displacement of the fluorophore due to twisting, can be replaced by a definite integral and evaluated to yield

$$
\begin{gathered}
\Gamma(t) \cong A \sqrt{t}, \quad t \leq \tau_{1} \\
A=\frac{2 k_{B} T}{\pi b \sqrt{\eta C}}
\end{gathered}
$$

Thus, anisotropy due to twisting decays as $\exp \left(-t^{1 / 2}\right)$ and is inversely proportional to the square root of both viscosity, $\eta$, and the torsion constant, C. Similarly, at short times, equation [6], the timedependent angular displacement due to bending, is evaluated to give the simplified expression

$$
\Delta(t) \cong B(t)^{4} \sqrt{t} \quad t \leq \tau_{1}^{\prime}
$$


where $B(t)$ is a slowly varying function of time

$$
B(t)=\left(\frac{3.466 k_{B} T}{\left(\pi^{5} \eta E^{3} I^{3}\right)^{\frac{1}{4}}}\right) f\left(z_{\max }\right)^{\frac{1}{4}}
$$

and $z_{\max }$ is determined by iteration from the equation

$$
\mathrm{z}_{\max }(\mathrm{t}) \approx \mathrm{b}\left[\frac{5.024 \pi \eta}{\mathrm{EIt} f\left(\mathrm{z}_{\max }\right)}\right]^{\frac{1}{4}}
$$

Significantly, anisotropy decay due to bending varies only as $\exp \left(-t^{1 / 4}\right)$, a weaker time dependence than for twisting.

Barkley and Zimm discuss some of the physical implications of their model at length. They explore the relative contribution of bending vs. torsion to the observed fluorescence depolarization by assuming certain reasonable values for the torsion and bending constants. First they calculate the anisotropy after $100 \mathrm{nsec}$ with twisting motions of the helix entirely suppressed, $\Gamma(t)=0$. Surprisingly, $r=0.379$ using this assumption. Since $r=0.4$ is attainable only for a rigid, immobile system, this calculation indicates that bending is expected to contribute very little to the overall anisotropy decay. For real measurements of DNA-ethidium bromide complexes, values of $\mathrm{r}$ greater than 0.37 are generally never observed even at the earliest times (Millar et al., 1980; Millar et al., 1982; Thomas et al., 1980; Thomas and Schurr, 1983; Wu et al., 1988). With bending suppressed, $\Delta(t)=0, r=0.209$ after 100 nsec. This value is roughly that which is observed in FPA experiments at long times (Millar et al., 1980; Millar et al., 1982; Thomas et al., 
1980; Thomas and Schurr, 1983; Wu et al., 1988). This calculation demonstrates the importance of torsional deformation compared to bending motions in the overall fluorescence anisotropy decay.

The insensitivity of FPA to high frequency bending modes is in part a consequence of the orientation of the ethidium dipole with respect to the helix axis. A reporter molecule is a sensitive probe of a particular molecular reorientation only to the extent that its dipole moment has some component which is perpendicular to the rotation axis of that depolarization mode. Thus, a dye with transition moment parallel to the helix axis would report only depolarization due to bending since any twisting of the helix axis would leave its direction unchanged (Fig 2.3A). Similarly, a dye with transition moment perpendicular to the helix axis would be most sensitive to twisting, since any rotation of the helix axis would reorient the dye by an equivalent angular displacement (Fig 2.3B). The dye with dipole moment perpendicular to the helix axis is also somewhat sensitive to bending since it is still perpendicular to one of the molecular axes about which bending occurs. For ethidium bromide, the dipole is nearly perpendicular to the helix axis, forming an angle of $70^{\circ}$ (Hogan et al., 1979). This alignment makes it a less sensitive measure of DNA bending than of twisting. There is a second reason that FPA is insensitive to DNA bending, as stated earlier. The bending decay function, $\Delta(t)$, is intrinsically less sensitive as a measure of rotational motion since it varies only as $\exp \left(-t^{1 / 4}\right)$ compared to the $\exp \left(-t^{1 / 2}\right)$ dependence of $\Gamma(t)$. As $t$ gets large, $t^{1 / 4}$ increases in magnitude only slowly, and thus bending does not contribute in great degree to depolarization. 
Fig 2.3 The FPA experiment in the molecular coordinate system. Each rotational mode, tw, b1 and b2, corresponds to a depolarization mode. Twisting corresponds to a rotation about the tw axis.

Bending results from rotations about b1 or b2. The bold arrow represents the dipole moment of the reporter molecule. (A) The reporter molecule is aligned parallel to the long axis of DNA. Rotations about tw leave the orientation of the dipole unchanged. Thus, with reporter aligned parallel, anisotropy measurements probe only bending motions. (B) The reporter molecule is perpendicular to the long axis, similar to the case for ethidium. In this orientation, the reporter is most sensitive to twisting. However, it is still sensitive to rotations about b2, and thus bending can still contribute to depolarization. 

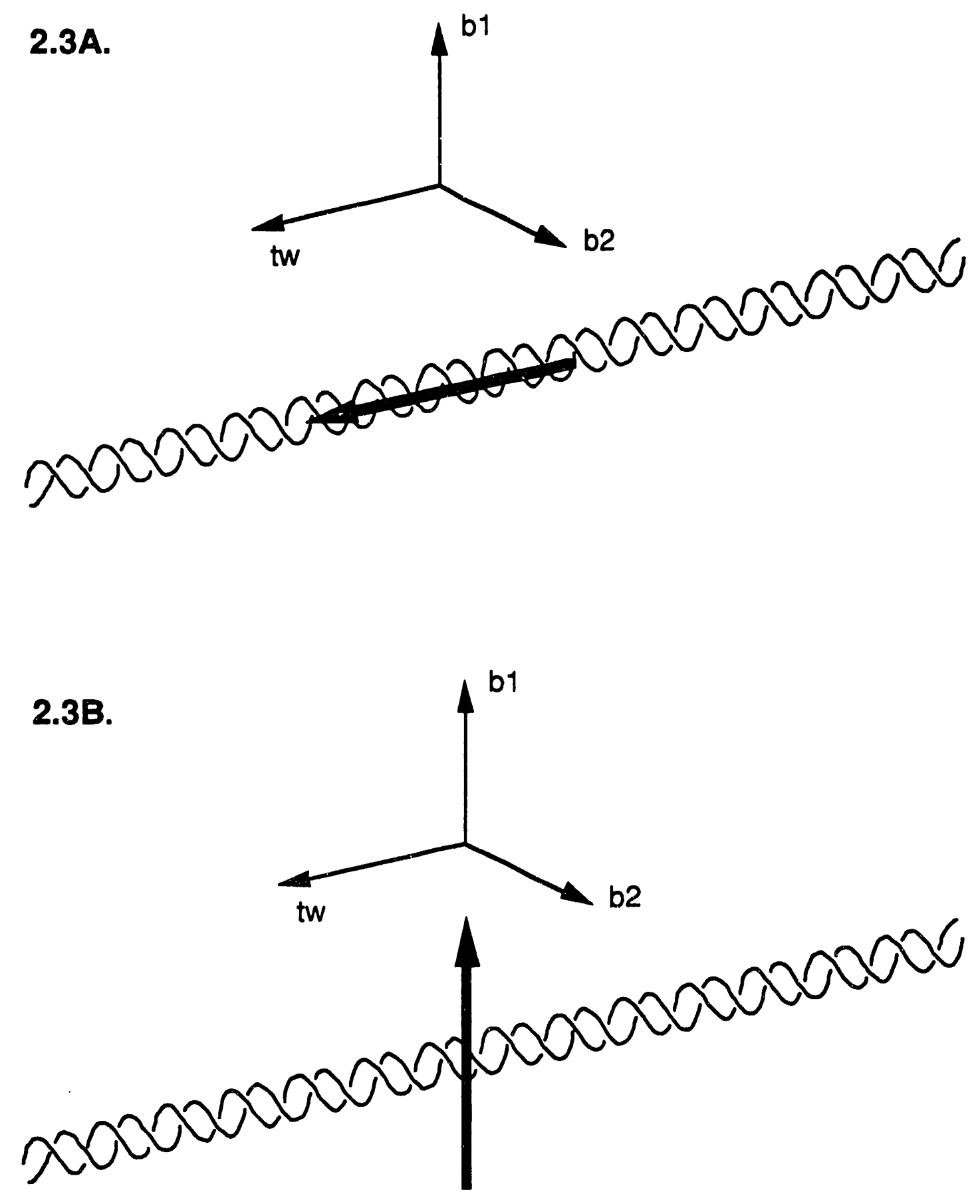
The Allison-Schurr intermediate zone formula. In their analysis of torsional dynamics, Allison and Schurr begin with a substantially different physical model for DNA (Allison and Schurr, 1979). They picture the helix as a linear sequence of identical rigid rods connected at their ends by torsion springs. Their model allows for arbitrary rod lengths, but the size of the rods can be distinguished by experiment (Thomas et al., 1980). The time course of rotational motion for this model can be divided into four characteristic time zones: zone (i) the initial exponential decay zone; zone (ii) the intermediate zone; zone (iii) the longest internal mode zone; and zone (iv) the uniform mode zone. The strength of the Allison-Schurr model lies in the explicit inclusion of all of these time regimes, most of which are important only for lengths of DNA smaller than one persistence length. Each of the zones has a physical significance for the overall rotation of the molecule. For DNAs longer than 3000 base pairs, only zone (ii) has relevance to the observed FPA decay. At the shortest times, the torsional rods in the Allison-Schurr model twist as independent, uncorrelated units, and therefore the anisotropy at these early times follows an exponential rate law. For time-resolved FPA measurements with $\geq 100$ psec resolution, zone (i) should be important only if torsion joints are separated by many ( $\geq 20)$ base pairs (Allison and Schurr, 1979; Thornas et al., 1980). As the torsional rods increase in size, their characteristic lifetime also increases. For a model in which the rod unit is a single base pair, the initial exponential decay zone should be apparent only at times much shorter than $25 \mathrm{psec}$ (Barkley and Zimm, 1979). In fact, Allison and Schurr initially argued in favor of a model with a rod 
length of 86 base pairs which followed the initial exponential decay law, based on their analysis of the early data of Wahl, et al. (1970) which was limited to $5 \mathrm{nsec}$ time resolution. Subsequent analysis of their own data led Schurr and his collaborators to reject this model of widely spaced torsional joints in favor of one with equivalent torsional springs separated by single base pairs (Thomas et al., 1980).

Zones (iii) and (iv) are incompatible with the time scale of the FPA measurements and so can also be ignored in analysis of ethidium fluorescence data. Zone (iii), the longest internal mode zone, corresponds to the twisting of the entire molecule about its helix axis, and can be discounted due to the long lifetime of this process. Zone (iv), the uniform zone mode, corresponds to times when all internal modes (twisting and bending) have relaxed, leaving only rotation of the entire macromolecule to achieve complete depolarization. For large DNA molecules, this rotational correlation time is expected to be milliseconds or longer (Allison and Schurr, 1979; Barkley and Zimm, 1979).

At intermediate times between zones (i) and (iii), the torsional oscillations of the rods are correlated, giving rise to the same physical behavior as that predicted by the elastic filament model of Barkley and Zimm. This is the time regime described by the intermediate zone in which the anisotropy decays as $\exp \left(-t^{1 / 2}\right)$. Mathematically, the anisotropy decay formula deduced by Allison and Schurr for this zone is identical to that given by Barkley and Zimm with bending suppressed, $\Delta(t)=0$, and with the simplified expression for $\Gamma$ at short times: 


$$
r(t)=r_{0}\left(A_{1}+A_{2} \exp [-\Gamma(t)]+A_{3} \exp \left[\frac{\Gamma(t)}{4}\right]\right) .
$$

An important difference between these two models is the treatment of DNA bending. A mathematical description of FPA data which ignores bending will tend to underestimate the torsional stiffness of the DNA, since it assumes that all of the observed fluorescence depolarization is due to twisting. In fact, bending does make some contribution to the anisotropy decay, however small. Schurr and his collaborators have argued, however, that the Barkley-Zimm treatment overestimates the bending contribution to depolarization (Fujimoto and Schurr, 1990; Schurr, 1984; Shibata et al., 1984; Thomas and Schurr, 1983). Schurr has concludid that the Barkley-Zimm model for bending deformations is valid only if axial tumbling makes no contribution to the depolarization (Schurr, 1984; Shibata et al., 1984)

Over the $10^{2}$ nsec time scale of the FPA measurements, one might argue that the helix axis is essentially immobile and that the bending approximations of the Barkley-Zimm model are appropriate. In any case, Schurr and his collaborators have stated that the inclusion of bending in their FPA analysis can be accounted for merely by applying a scale factor of between 1.0 and 1.9 to their measured torsion constants with a best estimate scaling factor of 1.35 (Fujimoto and Schurr, 1990; Shibata et al., 1984; Thomas and Schurr, 1983). It would seem that the best way to resolve this argument would be to develop a probe for measuring dynamics 
which is aligned parallel to the helix axis and so would be most sensitive to DNA bending.

\section{Materials and Methods}

Preparation of DNA samples. Topoisomers of negatively supercoiled plasmid pBR322 were prepared according to the method of Keller

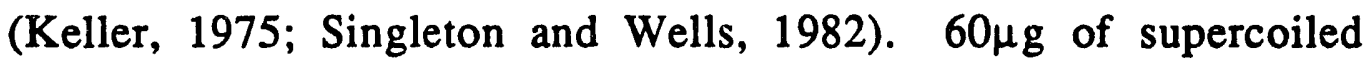
plasmid (approximately 10-20\% nicked starting material) was combined with $2.85,8.71,11.7$ or $14.8 \mathrm{mM}$ ethidium bromide (EtBr; Sigma; recrystalized in EtOH) in $1.5 \mathrm{ml}$ of buffer containing $50 \mathrm{mM}$ Tris $\cdot \mathrm{Cl}, \mathrm{pH} 7.6,50 \mathrm{mM} \mathrm{KCl}, 10 \mathrm{mM} \mathrm{MgCl}_{2}, 0.5 \mathrm{mM}$ dithiothreitol, 0.1 $\mathrm{mM}$ ethylenediaminetetraacetic acid (EDTA), and $30 \mathrm{mg} / \mathrm{ml}$ acetylated BSA. A relaxed sample (no EtBr) was prepared in a similar buffer without $\mathrm{MgCl}_{2}$ but containing $150 \mathrm{mM} \mathrm{KCl}$. This buffer is identical to that used for fluorescence anisotropy measurements. This sample served as a reference, defining $L k_{0}$ under ionic conditions very similar to that in vivo. Excess calf thymus topoisomerase I (Bethesda Research Labs), $4 \mathrm{U} / \mu \mathrm{g}$, was added to each reaction and incubated at $37^{\circ} \mathrm{C}$ for $4 \mathrm{hr}$. Samples were loaded into EtBr-CsCl density gradients (Sambrook et al., 1989) and spun to equilibrium in a VTi65 rotor $(55,000 \mathrm{rpm}$ for $18 \mathrm{hr}$ at $18^{\circ} \mathrm{C}$ ). The lower, supercoiled band was removed from the gradients with 18 gauge needles, extracted repeatedly with 20xSSC-saturated isopropanol to remove traces of ethidium, and EtOH precipitated twice. After measuring the concentration of DNA by absorption $\left(A_{260} / A_{280}=1.8\right.$ to 1.9$)$, the samples were again precipitated with alcohol and resuspended to a final concentration of $400 \mu \mathrm{g} / \mathrm{ml}$ in $\mathrm{TE}$ 
buffer (10 $\mathrm{mM}$ Tris.Cl, $\mathrm{pH} 8.0,1 \mathrm{mM}$ EDTA). The final product was $>95 \%$ intact form I plasmid.

Positively supercoiled pBR322 was prepared in vivo by treating strain AS19 (pBR322) with novobiocin, according to the protocol of Lockshon and Morris (Lockshon and Morris, 1983). AS19 is an $E$. coli B derivative which is particularly permeable to a wide range of small hydrophobic molecules (Sinden et al., 1980) including novobiocin. Novobiocin blocks the catalytic action of the enzyme DNA gyrase by binding efficiently to the ATPase site on the enzyme (Sugino et al., 1978). Cells were grown in a rich medium (LC (Sambrook et al., 1989)) containing $50 \mu \mathrm{g} / \mathrm{ml}$ ampicillin to mid-log phase $\left(\mathrm{OD}_{600}=0.4\right.$ to 0.5 as measured on a Bausch and Lomb Spectronic 21). Novobiocin $\left(8 \mathrm{mg} / \mathrm{ml}\right.$ in deionized, distillied $\mathrm{H}_{2} 0$ ) was added to a final concentration of $80 \mu \mathrm{g} / \mathrm{ml}$, and the cultures were incubated at $37^{\circ} \mathrm{C}$ on a gyratory shaker for another $30 \mathrm{~min}$. Plasmid DNA was isolated according to the standard alkaline-SDS lysis protocol (Sambrook et al., 1989) and banded twice on $\mathrm{EtBr}-\mathrm{CsCl}$ equilibrium density gradients. The final work-up procedures were carried out as for the negative topoisomers.

Phage lambda DNA for use in the sucrose viscosity experiments was purchased from BRL and stored in TE buffer.

Characterization of topoisomer distributions. The center of the linking number distribution for each sample was determined by the band counting method (Keller, 1975). DNA was run in $1.8 \%$ agarose gels in 0.5x TPE buffer (Sambrook et al., 1989) at $2 \mathrm{~V} / \mathrm{cm}$ for 16 to $20 \mathrm{~h}$. Chloroquine (Sigma) was added to the same final 
concentration in both the gel and the buffer to aid in the resolution of topoisomers, and buffer was recirculated during the run. The center of each linking number distribution was determined relative to the center of the relaxed distribution.

Samples for spectroscopy. Lambda DNA was prepared at a concentration of $300 \mu \mathrm{M}$ in base pairs $(200 \mu \mathrm{g} / \mathrm{ml})$ in $0.5 \mathrm{x}$ Spect Buffer (1x SB = $150 \mathrm{mM} \mathrm{KCl}, 50 \mathrm{mM}$ Tris, $\mathrm{pH} 8.0,1 \mathrm{mM}$ EDTA) with varying dilutions of a $60 \%$ sucrose stock (Sigma, grade1). Sucrose concentrations were measured both before and after FPA using a Bausch and Lomb refractometer. The relative viscosity, $\eta_{\text {rel }}$, was determined at the the experimental temperature, $23.5^{\circ}$, by a linear interpolation of the published values at $20^{\circ}$ and $25^{\circ} \mathrm{C}$. This approximation should not affect our interpretation of the data since, unlike the absolute viscosity, $\eta_{\text {rel }}$ varies only weakly with temperature. Topoisomer samples were diluted to $300 \mu \mathrm{M}$ in base pairs in 1x SB. Recrystalized $\mathrm{EtBr}$, dissolved in $1 \mathrm{xSB}$, was added to all samples to a final concentration of $2 \mu \mathrm{M}$.

Time-resolved fluorescence polarization instrumentation. Timeresolved FPA utilizes a technique known as Time Correlated Single Photon Counting or TCPC. Conceptually, this experiment is identical to a fluorescence depolarization experiment, except that far fewer than one fluorescent photon per laser excitation pulse is collected. The time between the laser pulse and the fluorescent photon can be measured with great accuracy, and a histogram of the number of photons per time interval is developed after many laser pulses. This 
histogram is equivalent to the fluorescence decay curve of the sample. The advantage of operating in the single photon counting mode is that the time between laser pulse and fluorescence can be measured with an accuracy of several picoseconds. The disadvantage compared to a steady state measurement is that the data collection takes longer and a great deal of the signal normally available is not utilized.

The TCPC instrument is shown in Fig 2.4. An argon ion laser, mode-locked at $514 \mathrm{~nm}$ with an $82 \mathrm{MHz}$ repetition rate (Spectra Physics model 2440-15, output pulse width of approximately 100 psec) was used to synchronously pump a cavity-dumped dye laser (Spectra Physics model 4500) containing the dye Rhodamine R6G. The output pulses from the dye laser $(6 \mathrm{psec})$ were tuned to $552 \mathrm{~nm}$ and the adjustable cavity-dumped repetition rate was typically set to $4 \mathrm{MHz}$. These pulses were passed through a vertical polarizer and into the sample.

Fluorescence was collected at right angles to the excitation beam and passed through an adjustable polarizer which was oriented either parallel or perpendicular to the exciting light. For most samples, data were also collected with the polarizer set to $54.7^{\circ}$, an angle which eliminates the rotational component of the decay and thus yields the fluorescence decay curve for the sample. After focusing by a camera lens, the light passed through an interference filter and a quarter-wave plate. The interference filter (Omega Optical, Brattleboro, VT) eliminates Rayleigh (elastically) scattered light $\left(O D_{550}=6\right)$ which arises primarily from the cuvette surfaces and which would otherwise overwhelm the fluorescence 
Fig 2.4. The photon counting instrument. The $514 \mathrm{~nm}$ line from a mode-locked Argon-ion laser is used to pump a dye laser containing Rhodamine $6 \mathrm{G}$, and the dye laser is tuned to $552 \mathrm{~nm}$. The pulses from the dye laser are cavity dumped at a rate of $4 \mathrm{MHz}$. Part of the beam is split off to activate a photodiode. This pulse, after a delay, serves as the stop signal for the time-amplitude converter (TAC) circuitry. The main beam is passed through a vertical polarizer and into the sample. Fluorescence is detected at right angles to the exciting beam, passing through an analyzer which is set either parallel or perpendicular to the excitation polarization. After focusing through a lens, light is passed through an interference filter which removes both Rayleigh and Raman scattering, then through a quarter wave plate which circularly polarizes the light and reduces the polarization sensitivity of the detector. Photons strike the microchannel plate, giving rise to a cascade of electrons at the photomultiplier tube. The output is amplified and passed to a discriminator which rejects signal below and above certain threshold levels. The pulse initiates a voltage ramp in the TAC which is stopped by the delayed signal from the photodiode. The data is stored in a multichannel data analyzer, and, at the completion of a run, is transferred to a computer for storage and analysis. 


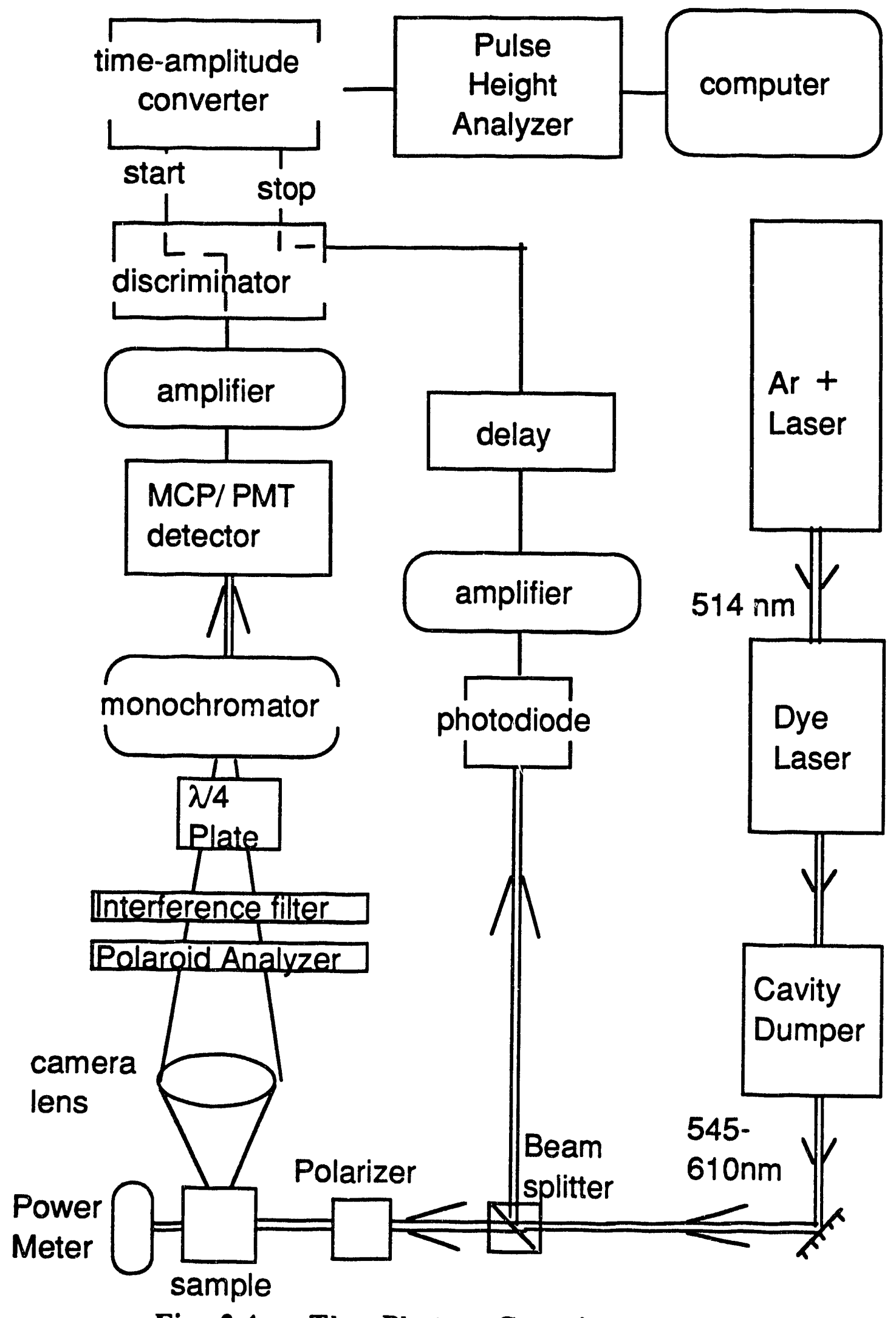

Fig 2.4. The Photon Counting Instrument 
signal. It also cuts out Raman (inelastically) scattered light $\left(O D_{685}=\right.$ 4), which in $\mathrm{H}_{2} \mathrm{O}$ is shifted $3600 \mathrm{~cm}^{-1}$ from the excitation wavelength. The quarter-wave plate is oriented at approximately $45^{\circ}$ to both the perpendicular and parallel fluorescence emission and thus circularly polarizes the light before it hits the detector. This serves to minimize the polarization sensitivity of the detection system.

Photons strike a red-sensitive microchannel plate photomultiplier tube (Hamamatsu MCP-PMT \#R2809U-11) resulting in the ejection of photoelectrons from the MCP-PMT. These energetic electrons are accelerated through a high potential, and a secondary cascade of electrons, resulting from collisions by the photoelectron with the walls of the MCP-PMT, is detected at the anode. The advantage of an MCP-PMT over conventional PMTs is better time resolution. The trajectory of photoelectrons in the MCPPMT is constrained to follow one of several thousand capillary tubes (the microchannels) which are on the order of $10 \mu \mathrm{m}$ wide. By minimizing the path length differences between photoelectrons, the transit time spread of the detector, and hence its time resolution, can be decreased. Essentially, the transit time spread is a measure of the uncertainty as to when a photon actually strikes the MCPPMT. For the Hamamatsu MCP-PMT, the transit time spread is about $55 \mathrm{psec}$ wide. The actual uncertainty can be reduced, in the case where the transit time spread is a well-understood function, to 5-10 psec by a deconvolution of the data. The output from the MCP-PMT is amplified by a Hewlett Packard 8447F amplifier with a bandwidth of $1.3 \mathrm{GHz}$ and then sent to a Tenneco constant fraction 
discriminator. The discriminator checks that each pulse is within a certain amplitude window. The lower threshhold limit eliminates some signal due to thermal ionizations in the MCP-PMT, and the upper threshhold selects against large spikes due to either voltage surges or to two photon events at the MCP-PMT. The signal then initiates a voltage ramp in the time to amplitude converter (TAC, Ortec 457) which is stopped by a delayed signal from the excitation laser pulse. A Northern 770 pulse height analyzer stores the data to create a histogram.

This "backwards" mode of operation (start signal from the fluorescent photon, stop from the laser pulse) is useful in TCPC since it decreases the dead time in the system and the skewing of data which would result (see below). There is a requisite dead time for each start in order to eliminate noise in the system. To eliminate two photon events, less than $1 \%$ of the input laser pulses result in a fluorescent photon in a photon counting experiment. The TAC electronic circuitry would be needlessly activated, and the dead time of the system correspondingly increased, if a voltage ramp were initiated by every laser pulse. By making the fluorescent photon the start signal and the delayed laser pulse the stop signal, the backwards mode enhances the throughput of the system.

Data analysis. In the experiments reported here, the instrument response function was sufficiently narrow $(60 \mathrm{psec})$ that the observed anisotropy decays were not distorted by convolution for the times of interest $(\geq 100 \mathrm{psec})$. Several corrections were necessary, however, to account for systematic errors in the data due 
to instrumental bias. The first correction was for the $\mathrm{g}$ factor, or polarization sensitivity of the detection system. For a rapidly tumbling small molecule such as $\mathrm{EtBr}$, the total fluorescence intensity after complete reorientation should be identical in parallel and perpendicular modes in the absence of a polarization sensitivity by the detector. The measured intensity ratio $L / I_{\text {" }}$ for a small molecule at long times is the $\mathrm{g}$ factor or polarization sensitivity for the instrument. The $g$ factor was measured with a solution of free $\mathrm{EtBr}$ (rotational correlation time of approximately 100 psec; fluorescence lifetime approximately $2 \mathrm{nsec}$ ) by comparing total fluorescence intensities $I_{\perp}$ and $I_{\|} 1 \mathrm{nsec}$ after the laser pulse.

A second correction was made for variations in laser light intensity striking the sample. Since the anisotropy function is a ratio, small errors in total signal intensity are magnified in the final data. To correct for this effect, the total power striking the sample was monitored with a power meter (Spectra Physics model 4041). The output of the meter was digitized with a voltage to frequency converter and counted with an Ortec 778 Dual Counter. The light normalization factor $L_{f}$ could be expressed as the ratio of perpendicular to parallel intensities: $\mathrm{L}_{\mathrm{f}}=\mathrm{L}_{\perp} / \mathrm{L}_{\|}$. Typically, $\mathrm{L}_{\mathrm{f}}$ varied randomly around 1.0 .

A final correction was made for the dead time of the instrument which resulted in a systematic loss of photons in the parallel mode where the count rate was high. The limiting dead time $\mathrm{d}$ in the system derived from the $4.5 \mu \mathrm{sec}$ dead time of the pulse height analyzer. The observed average time between photons, $t_{a v g}$, is just the inverse of the count rate, which in these experiments 
is approximately $4 \times 10^{3} \mathrm{cps}$ for $\mathrm{I}_{\|}$and $2 \times 10^{3}$ for $\mathrm{L}$. The fraction of photons missed is just $d /\left(t_{a v g}+d\right)$. Since $t_{a v g}$ is smaller for $I_{/ l}$, the data is systematically skewed to lower anisotropy values by missing the $I_{/ /}$counts. Typically, $5 \%$ of the parallel photons and $2.5 \%$ of the perpendicular photons were missed due to the pulse height analyzer dead time.

The total fluorescence decay was determined from data sets with the analyzer rotated to $54.7^{\circ}$. The total fluorescence was then deconvoluted from the instrument response function and fitted to a sum of exponentials to determine the contribution of bound and free ethidium bromide to the total signal

$$
s(t)=A\left[f \exp \frac{t}{\tau_{b}}+(1-f) \exp \frac{t}{\tau_{f}}\right]
$$

where $\tau_{b}$ and $\tau_{f}$ are the lifetimes of intercalated and free dye, respectively, and $f$ is the fraction of the initial amplitude $A$ due to bound fluorescence.

The anisotropy decay, $r(t)$, was formed for each channel in the fluorescence decay histogram:

$$
r(t)=\frac{g L_{f} I_{/ /}(t)-I_{\perp}(t)}{g L_{f} I_{/ /}(t)+2 I_{\perp}(t)}
$$

This decay curve was then fitted to equation [3] using a non-linear least squares approach based on Marquardt's algorithm (Marquardt, 1963), with the simplified expressions for $\Gamma(t)$ and $\Delta(t)$, and with two adjustable parameters, $r_{0}$, the initial anisotropy, and $C$, the 
torsion constant. Other parameters were taken from the literature as described in the text below.

\section{Results}

Initial characterization of samples. Gel analysis of the topoisomer samples demonstrates that after relaxation with topoisomerase I and banding in an $\mathrm{EtBr}-\mathrm{CsCl}$ equilibrium gradient, the DNA was >95 $\%$ supercoiled (Fig 2.5). In this gel, samples were run in the presence of approximately $0.5 \mu \mathrm{g} / \mathrm{ml}$ ethidium in both the gel and the buffer. This concentration of ethidium overwinds all of the DNA samples so that the plasmids migrate as positive supercoils. Under these conditions, samples which are initially more positively supercoiled run faster than those which were negatively supercoiled in the absence of ethidium. Thus, even though the same plasmid, pBR322, is run in each lane, the samples band at significantly different position due to their initial superhelical density. After the FPA experiment, including multiple precipitations, the DNA was still $>95 \%$ intact (Fig 2.6). Control experiments indicate that irradiation with 10- to 100 -fold more light than in a typical fluorescence experiment resulted in essentially no nicking of the DNA (data not shown). Thus, the small amount of observed nicking was probably due to handling of samples before and after irradiation.

The linking difference, $\Delta \mathrm{Lk}$, of the various samples was determined by running the DNA in gels containing from 0 to 17.5 $\mu \mathrm{g} / \mathrm{ml}$ chloroquine. Chloroquine intercalates between base pairs, unwinding the DNA. For negatively supercoiled DNA, at low levels of 
This page for number sequence only. 
This page for number sequence only. 
Fig 2.5. Topoisomer samples after final CsCl-EtBr purification. DNA samples were run in a $1 \%$ agarose gel at $2 \mathrm{~V} / \mathrm{cm}$ in the presence of 1 $\mu \mathrm{g} / \mathrm{ml}$ ethidium. At this concentration of intercalator, the samples bind a limiting amount of ethidium and are thus maximally overwound. Under these conditions, negatively supercoiled DNA migrates more slowly than positively supercoiled DNA. This photograph demonstrates that the samples were greater than $95 \%$ covalently closed before fluorescence spectroscopy. Lanes represent: $\sigma=+0.042$ (A); $\sigma=0.0$ (B); $\sigma=-0.025$ (C); $\sigma=-0.045$ (D); $\sigma$ not determined (E); $\sigma=-0.069(\mathrm{~F}) ; \sigma=-0.096(\mathrm{G}) ; \sigma=-0.123(\mathrm{H})$; and starting material (I). Note the particularly broad smear of the positive sample. The reason for the breadth of this distribution is explained in the text. 
Fig 2.5. Topoisomer samples run in an agarose gel in the presence of $\mathrm{EtBr}$
$\begin{array}{lllllllll}A & B & C & D & E & F & G & H & \text { I }\end{array}$

$N$
$D$

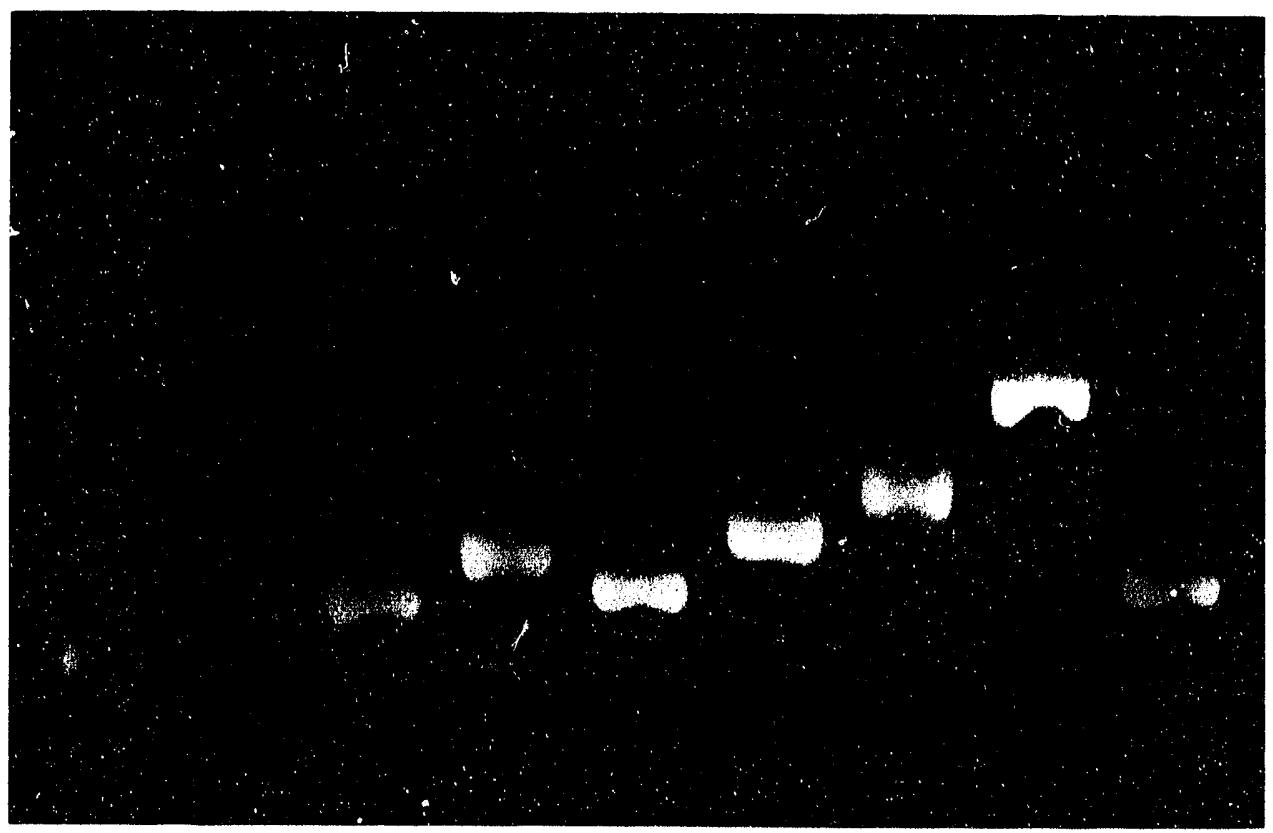


Fig 2.6. Agarose gels which resolve the topoisomer species in each sample. (A) A gel run in the absence of chloroquine. Only samples near the relaxed linking number are resolved under these conditions. 17 bands are distinguishable in the positively supercoiled sample before the main smear occurs. (B) A gel run in the presence of $5 \mu \mathrm{g} / \mathrm{ml}$ chloroquine, which resolves the topoisomers of some of the intermediate superhelix densities. It is important to note that the samples shown here do not overlap significantly in the distributions of topoisomer bands. 
Fig 2.6A. Topoisomer Samples without Chloroquine

Superhelix Density

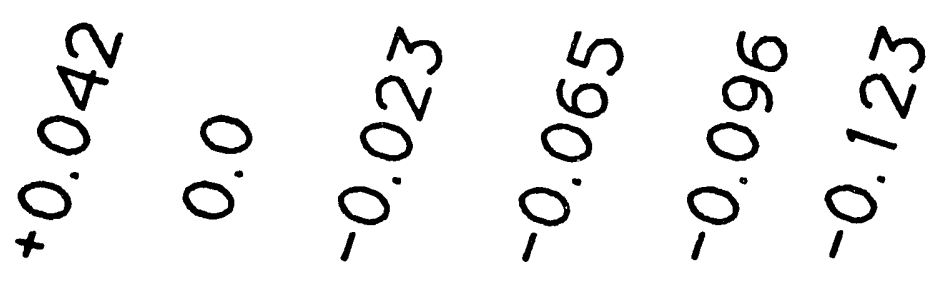


Fig 2.6B. Topoisomer Samples with

$5 \mu \mathrm{g} / \mathrm{ml}$ Chloroquine

Superhelix Density

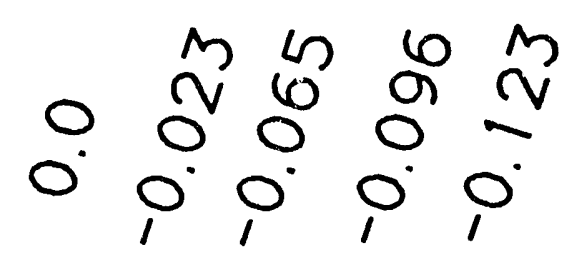

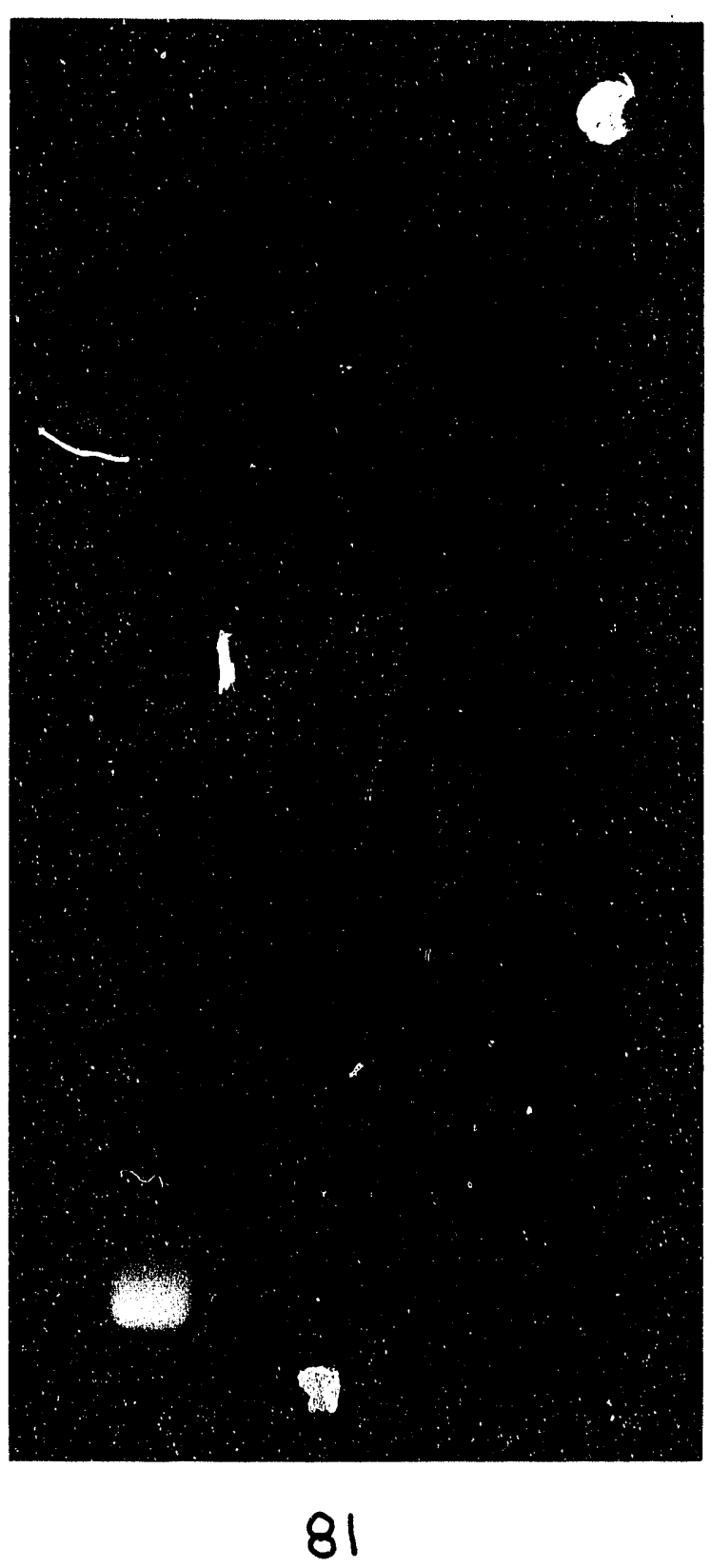


of chloroquine binding, this unwinding increases the hydrodynamic radius of the molecule. Agarose gels are sensitive to this increase in size, and so covalently closed, negatively supercoiled plasmids decrease in mobility upon addition of chloroquine at low concentrations. At some point, depending on the initial underwinding of the DNA, the intercalator begins to overwind the DNA, and the plasmid runs faster in the gel. The population of molecules is positively supercoiled compared to a plasmid sample relaxed to equilibrium in the presence of the same concentration of chloroquine. Thus, titration of supercoiled DNA with an intercalator initially decreases the DNA mobility to a minimum and then increases mobility upon positive supercoiling.

Several of the topoisomer gels used to determine the superhelicity of the samples are shown in Fig 2.6. In general, negative topoisomers, prepared according to the protocol outlined in Materials and Methods, form a Gaussian distribution about the mean linking number of the sample (Depew and Wang, 1975; Pulleyblank et al., 1975). (Note that the average linking number is not necessarily an integer, although the linking number for any particular band can only have integral values; the average linking number is defined by the center of the Gaussian distribution which can fall between two bands (Peck and Wang, 1981; Wang, 1979). This correction makes only a slight change in the a erage linking number and is not important for the analysis of the data presented here.) As a catalyst, topoisomerase I interconverts the various topoisomers in the sample by nicking and resealing the DNA until a dynamic equilibrium is established. Since the energy difference 
between topoisomers is small, the equilibrium state contains a distribution which reflects the Boltzmann distribution of energy (Depew and Wang, 1975; Pulleyblank et al., 1975). One point is of interest concerning the positively supercoiled DNA sample. It is clear from the gel without chloroquine that the collection of topoisomers in the positively supercoiled sample does not follow a Gaussian distribution (Fig 2.6). This non-Gaussian distribution results from the method of preparation of the positively supercoiled sample. The distribution of topoisomers in the positive sample is representative of the kinetic distribution of linking numbers found in $E$. coli after inactivation of DNA gyrase with novobiocin. Significantly, all of the bands in the positively supercoiled sample run faster in the gel than does the relaxed sample. Although the distribution of positive topoisomers is broad, it does not overlap with any other sample.

Determining the superhelicity of negatively supercoiled samples is performed by measuring their linking deficit, $\Delta \mathrm{Lk}$, compared to the relaxed sample. The linking deficit can be measured by simply counting bands in the ladders formed by the topoisomers starting from the center of the relaxed sample. Adjacent bards differ in $L k$ by -1 going from slower to faster mobility for the negatively supercoiled state. The number of bands between the primary relaxed topoisomer and a particular topoisonier of interest is equal to $\Delta \mathrm{Lk}$. The number of steps to the strongest band in a lane is the average $\Delta \mathrm{Lk}$ of that sample. The specific linking difference, $\sigma$, is the linking deficit $\Delta \mathrm{Lk}$ normalized by the linking number $L_{k}$ of the relaxed molecule: $\sigma=\Delta L k / L k_{0}$. 
$\mathbf{L k}_{\mathrm{o}}$ is estimated under standard physiological conditions to be $\mathrm{N} / 10.5$, where $\mathrm{N}$ is the total number of base pairs. All measurements of $\sigma$ therefore relate to the set of conditions which define $\mathrm{Lk}_{0}$.

A measurement of $\sigma$ for the positively supercoiled sample gives only a lower bound estimate of the superhelicity. As the plasmid becomes overwound, it decreases in hydrodynamic radius. The positively supercoiled DNA migrates faster than relaxed or negatively supercoiled DNA under all conditions. The gel cannot resolve the individual topoisomers within this population (Fig 2.6). For the positively supercoiled sample, $L k$ increases by 1 for each band of increasing mobility in the gel. A lower bound of $\sigma \geq 0.042$ can be estimated by counting bands up to the point where the gel fails to resolve any further. At this point, $\Delta \mathrm{Lk}=+17$, and the bulk of the topoisomer distribution is unresolved. The value of $\sigma$ for this sample is significantly greater than that reported by $\mathrm{Wu}$ et al. (1988), who estimate $\sigma \geq 0.03$ for their samples prepared by a similar method.

Characterization of fluorescence lifetimes. Measurement of the fluorescence lifetimes of the DNA-ethidium bromide samples is a sensitive measure of the amount of free dye. Having a low level of free dye is critical for the measurement of anisotropy since ethidium in solution tumbles rapidly, with a correlation time of approximately $100 \mathrm{psec}$. A rapid decrease in anisotropy as a result of the tumbling of free dye might be incorrectly ascribed to motions of the DNA helix, if it constitutes a substantial portion of the 
observed signal. Also, free ethidium has a significantly shorter fluorescence lifetime than does intercalated dye, so it skews the anisotropy data preferentially at early times.

The fluorescence lifetimes of the topoisomer samples are given in Table 2.1. The observed lifetimes of approximately 23.5 and 2.0 nsec are in good agreement with the literature values for ethidium bound to DNA and free in solution, respectively (Millar et al., 1982). The analysis of fluorescent lifetimes is based on data taken with a polarizer between the sample and the detector set at $54.7^{\circ}$ and fitted to the following equation:

$$
f(t)=f_{0}\left[A \exp \left(\frac{t}{\tau_{b}}\right)+(1-A) \exp \left(\frac{t}{\tau_{f}}\right)\right]
$$

The quality of these fits can be judged by the $\chi_{\text {red criterion. For }}^{2}$ single photon counting experiments, the data are expected to follow Poisson statistics (O'Connor and Phillips, 1984). For a Poisson process, the average deviation from the true mean in any time interval $\Delta t$ goes as the square-root of the mean number of events in that interval. Thus, the relative error in a measurement decreases as $1 / \sqrt{\mathrm{N}}$, where $\mathrm{N}$ is the number of observations in a given time interval. The $\chi_{\text {red }}^{2}$ is simply the sum of the squares of the errors over all of the time intervals, weighted by the number of photons observed in each interval

$$
\chi_{\text {red }}^{2}=\frac{1}{\mathrm{dfe}} \sum_{\mathrm{n}} \frac{\left(\sigma_{\mathrm{n}}-\sigma_{\mathrm{f}}\right)^{2}}{\sigma_{\mathrm{n}}}
$$

where $\sigma_{n}$ is the number of photons in time interval $n, \sigma_{f}$ is the calculated (mean) number of photons based on the fitted curve of 
Table 2.1. Fluorescence Lifetimes of Topoisomer Samples from data taken with analyzer set at $54.7^{\circ}$

\begin{tabular}{|c|c|c|c|c|c|c|}
\hline Sample $^{a}$ & $\underline{\mu}^{b}$ & $\underline{\tau_{b}^{c}}$ & $\tau_{f}^{d}$ & $\mathbf{A}^{\mathbf{e}}$ & $\chi_{\text {red }}^{2}$ & $\underline{\Phi b}^{g}$ \\
\hline linear & 175 & 23.4 & 1.9 & 0.94 & 1.27 & 0.995 \\
\hline+0.042 & 175 & 23.6 & 1.9 & 0.93 & 1.21 & 0.994 \\
\hline 0.00 & 175 & 23.3 & 1.5 & 0.89 & 1.22 & 0.992 \\
\hline-0.025 & 175 & 23.4 & 1.1 & 0.90 & 1.27 & 0.994 \\
\hline-0.069 & 175 & 23.8 & 3.0 & 0.95 & 1.23 & 0.993 \\
\hline-0.096 & 175 & 23.5 & 1.1 & 0.91 & 1.29 & 0.996 \\
\hline-0.123 & 175 & 23.6 & 2.4 & 0.96 & 1.28 & 0.995 \\
\hline linear & 5 & 23.5 & 2.3 & 0.96 & 1.18 & 0.996 \\
\hline+0.042 & 5 & 23.5 & 1.7 & 0.95 & 1.29 & 0.997 \\
\hline 0.00 & 5 & 23.3 & 1.4 & 0.92 & 1.26 & 0.995 \\
\hline-0.025 & 5 & 23.5 & 1.1 & 0.91 & 1.34 & 0.996 \\
\hline-0.069 & 5 & 23.6 & 1.6 & 0.95 & 1.26 & 0.996 \\
\hline-0.123 & 5 & 23.6 & 2.1 & 0.94 & 1.29 & 0.994 \\
\hline
\end{tabular}

a Superhelix density of sample.

$b$ Ionic strength in $\mathrm{mM}$.

c Fluorescence lifetime of intercalated ethidium in nsec.

d Fluorescence lifetime of free dye in nsec.

- Initial amplitude of bound fluorescence species at $t=0$.

$f$ Reduced chi-squared of two parameter fit to magic angle data.

8 Fractional yield of fluorescence from bound species. 
the data, and dfe is the number of degrees of freedom. Since the average error $\left(\sigma_{n}-\sigma_{f}\right)$ should be approximately $\sqrt{\sigma_{n}}$, and since for

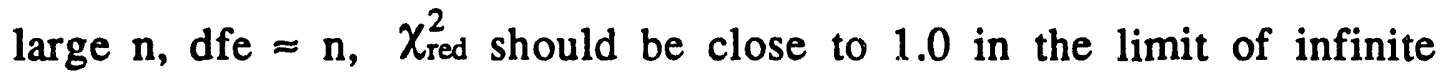
time of data collection. For our data, $\chi_{\text {red }}^{2}$ falls in the range 1.2 to 1.3 and is thus an acceptable fit to the data.

The binding constant of ethidium to DNA varies as a function of superhelicity (Bauer and Vinograd, 1970). For linear or nicked circular DNA, the binding constant $\mathrm{K}_{\mathrm{b}}=6.4 \times 10^{4} \mathrm{M}^{-1}$. For negatively supercoiled DNA, the binding constant is larger since intercalation of the drug relaxes some of the torsional and flexural strain imposed by underwinding of the helix. In terms of free energy, the binding of drug can be divided into two components, the intrinsic free energy of binding $\Delta G_{i}$, which is reflected in the binding constant to linear DNA, and the additional free energy of supercoiling $\Delta G_{s c}$, which is negative for negatively supercoiled DNAs. Thus, $\Delta G_{\text {tot }}=\Delta G_{i}+$ $\Delta G_{\text {sc. }}$ At the concentration of dye and DNA used in these experiments ( $2 \mu \mathrm{M}$ in dye and $300 \mu \mathrm{M}$ in base pairs), about $5 \%$ of the intercalator should be free in solution for linear DNA. Since free ethidium has a 20-fold lower quantum yield for fluorescence than does bound ethidium (LePecq and Paoletti, 1967), and since at 552 $\mathrm{nm}$, the absorbance cross section of free ethidium is reduced 3 -fold compared to bound dye (Bresloff and Crothers, 1975), the total fluorescence yield from free dye is much lower than that from the bound species. For each sample in the FPA experiments described here, the total yield of fluorescence from bound dye, $\Phi_{b}$, is greater than 99\% (Table 2.1). 
Positive supercoiling of the DNA should decrease the binding affinity of ethidium. At the concentration of DNA in these experiments, however, there is no detectable increase in iree dye for the positively supercoiled samples (Table 2.1). The fitted fluorescence data and the residual after the fit also demonstrate that there is no increase in the contribution of free ethidium bromide to the observed signal from the positively supercoiled sample in either the high salt or low salt conditions of the fluorescent measurements (Fig 2.7). This is an important conclusion for later interpretations of the anisotropy data, since the positively supercoiled DNA at low ionic strength seems to have different dynamic behavior from relaxed or negatively supercoiled DNA (as detailed below).

Sensitivity of anisotropy decay to changes in viscosity. Both models for twisting dynamics of DNA-ethidium complexes predict a decay function of the form

$$
r(t)=r_{0} \exp (-A \sqrt{t})
$$

where $A$ is

$$
\mathrm{A}=\frac{2 \mathrm{k}_{\mathrm{B}} \mathrm{T}}{\pi \mathrm{b} \sqrt{\eta \mathrm{C}}}
$$

and the other variables are as described in the Theory section above. As an experimental test of the sensitivity to small variations in C, FPA data was measured as a function of $\eta$ for lambda DNA (a linear phage DNA of approximately $50 \times 10^{3}$ base pairs). $\eta$ was systematically varied by adding sucrose to the DNA solution. The refractive index of the solution provides an accurate measurement 
Fig 2.7. The fluorescence decay from positively supercoiled samples with analyzer set to $54.7^{\circ}$. (A) Sample in low salt $(5 \mathrm{mM}$ Tris. $\mathrm{Cl}, \mathrm{pH} 8.0,0.5 \mathrm{mM}$ EDTA). (B) Sample in physiological salt (150 $\mathrm{mM} \mathrm{KCl}, 50 \mathrm{mM}$ Tris. $\mathrm{Cl}, \mathrm{pH} 8.0,1 \mathrm{mM}$ EDTA). Each panel shows the residual after fit (upper), the fitted fluorescence decay curve on a logarithmic scale (middle), and the instrument response function (lower). Note that the instrument response function spans only part of the fluorescence decay time scale. IRF measurements were typically made at a setting of $14 \mathrm{psec} / \mathrm{channel}$ whereas fluorescence data were routinely gathered at a setting of $75 \mathrm{psec} / \mathrm{channel}$. In this case, the IRF has a full-width, half-maximum of $60 \mathrm{psec}$. 
Fig 2.7A Positively Supercoiled pBR322 in $5 \mathrm{mM}$ Tris, $1 \mathrm{mM}$ EDTA

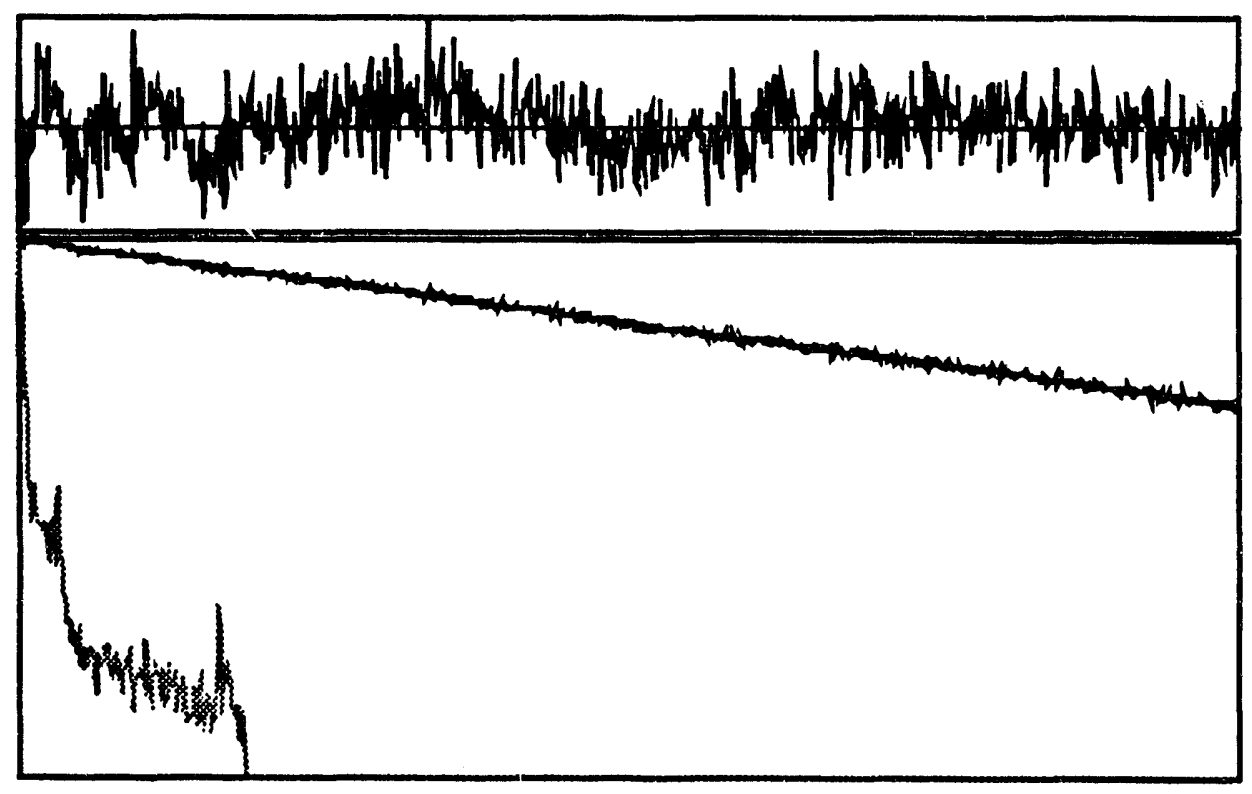

Fig 2.7B Positively Supercoiled pBR322 in $150 \mathrm{mM} \mathrm{KCl,} 50 \mathrm{mM}$ Tris

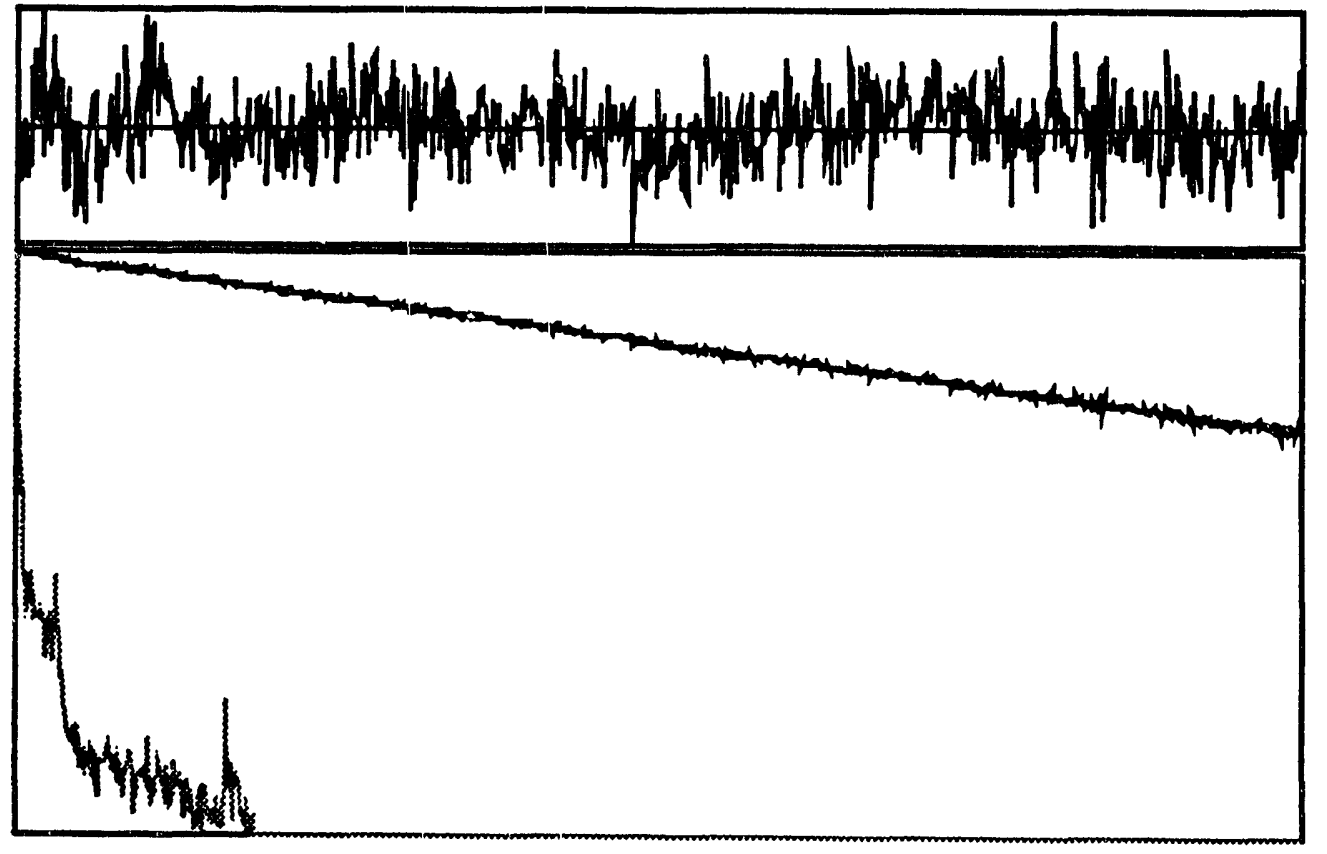

90 
of the sucrose concentration, and this concentration can be converted to a viscosity by using data compiled in the literature. In this experiment, it is assumed that the intrinsic torsion constant does not vary with sucrose and that all changes in the fit parameter A arise from the effects of viscosity. The data are shown in Table 2.2 and are analyzed by plotting $\left(1 / \eta_{\text {rel }}\right)^{1 / 2}$ vs. A in Fig 2.8. The correlation coefficient of the best-fit straight line is 0.99 . These results demonstrate that FPA measurements are quite sensitive to small changes in $\eta_{\text {rel }}$ and that the predicted linear function is observed.

Representative FPA data sets are shown for lambda DNA in the two extremes of sucrose concentration, $0 \%$ and 22\% (Fig 2.9). These decay curves are representative for all of the data discussed in later experiments. The envelope of the data set expands at long times as the anisotropy decreases. This effect is due to the lower total fluorescence at long times after the laser pulse. A weighted fit of the data compensates for this scatter and results in the fits shown by the solid lines.

The anisotropy decay curve has several interesting features. First, the initial anisotropy, $r_{0}$, does not approach the limiting theoretical value of 0.4 . This probably indicates substantial subnanosecond motions of the ethidium in its intercalation site (Barkley and Zimm, 1979; Millar et al., 1982). In general, $r_{0}$ ranges between 0.34 and 0.36 for all of the samples examined. The decay has a peculiar shape, with a rapid initial loss in anisotropy and a final leveling off at long times. This shape is due to the fact that twisting motions, which dominate the dynamic modes of DNA on the 
Table 2.2. Data for sucrose viscosity titration of lambda DNA in $75 \mathrm{mM} \mathrm{KCl}, 50 \mathrm{mM}$ Tris. $\mathrm{Cl}, \mathrm{pH} 8.0$

$\begin{array}{lll}\eta_{\text {rel }^{a}} & \frac{\sqrt{1 / \eta_{\text {rel }}}}{} & \hat{\theta}^{b} \\ 1.000 & 1.000 & 4.818 \\ 1.000 & 1.000 & 4.775 \\ 1.000 & 1.000 & 4.802 \\ 1.140 & 0.936 & 4.436 \\ 1.245 & 0.896 & 4.323 \\ 1.382 & 0.851 & 3.989 \\ 1.509 & 0.814 & 3.906 \\ 1.763 & 0.753 & 3.612 \\ 2.454 & 0.638 & 3.125\end{array}$

a Relative viscosity calculated from refractive index of sample calculated from refractive index reading and correcte for temperature using a linear interpolation of data from CRC Handbook of Chem. and Physics, 65th ed. and CRC Handbook of Biochem, $2^{\text {nd }}$ ed.

\footnotetext{
$b$ Fit Parameter $A=\frac{2 k_{B} T}{\pi b \sqrt{\eta C}}$ with symbols as defined in the text.
} 
Fig 2.8. Dependence of anisotropy decay on relative viscosity

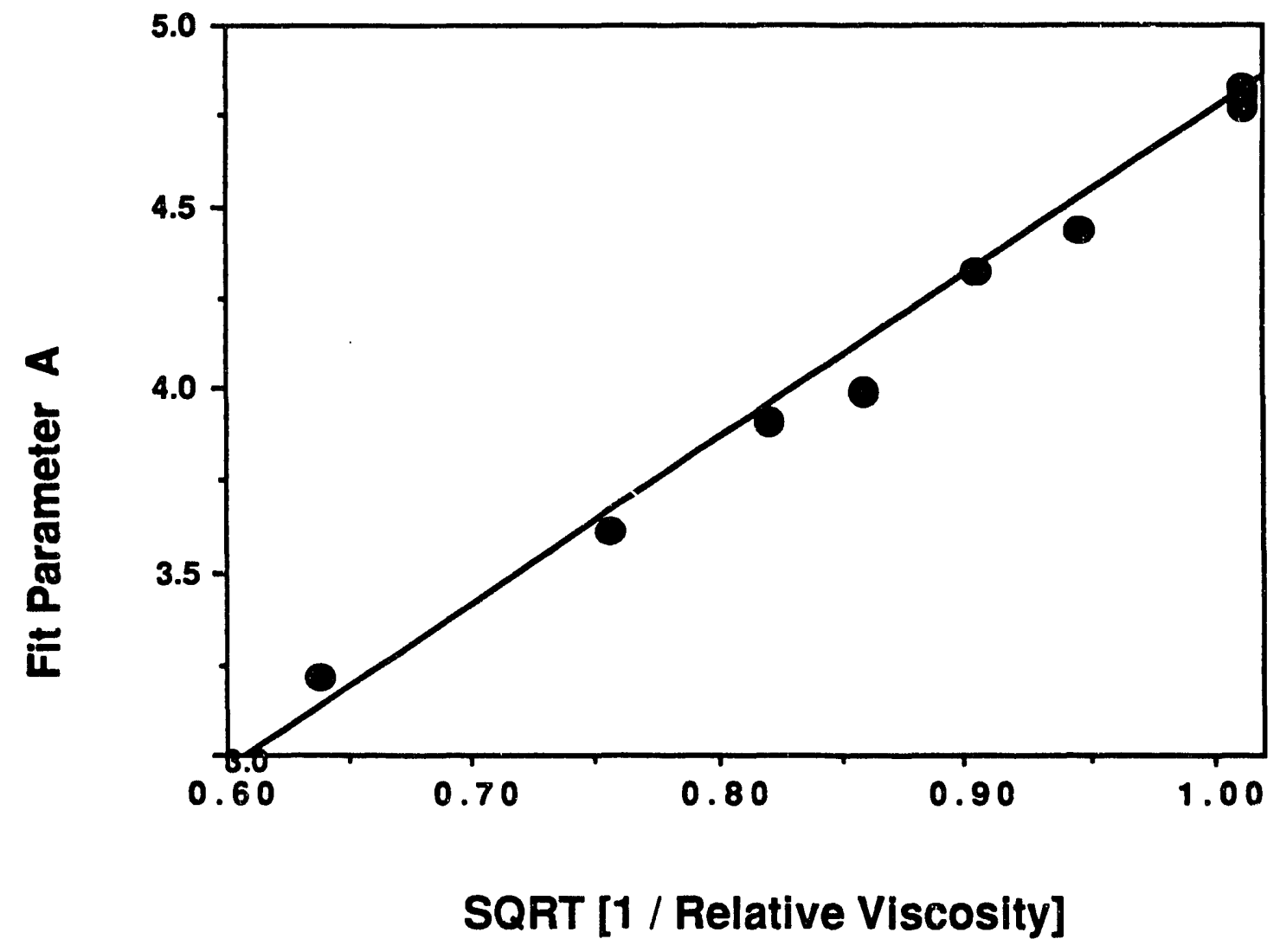

Fig 2.8. Plot of the data from Table 2.2. The data is in excellent agreement with the theory and demonstrates a sensitivity of the FPA measurements to changes in $\eta_{\text {rel }}$ of $10 \%$ or less. 
Fig 2.9. Anisotropy decay curves for lambda DNA in $0 \%$ and $24 \%$ sucrose. Lambda DNA at a concentration of $300 \mu \mathrm{M}$ in base pairs was mixed with ethidium at a concentration of $2 \mu \mathrm{M}$ in $75 \mathrm{mM} \mathrm{KCl}$, $25 \mathrm{mM}$ Tris. $\mathrm{Cl}, \mathrm{pH} 8.0,0.5 \mathrm{mM}$ EDTA without (A) or with (B) sucrose. Data were collected by modulating the analyzer between parallel and perpendicular modes for $5 \mathrm{~min}$ each over a period of $1 \mathrm{hr}$. Each data point represents the anisotropy value from the photons in a particular time interval (channel). 
Fig 2.SA LEmbda DNA In 0\% Sucroso

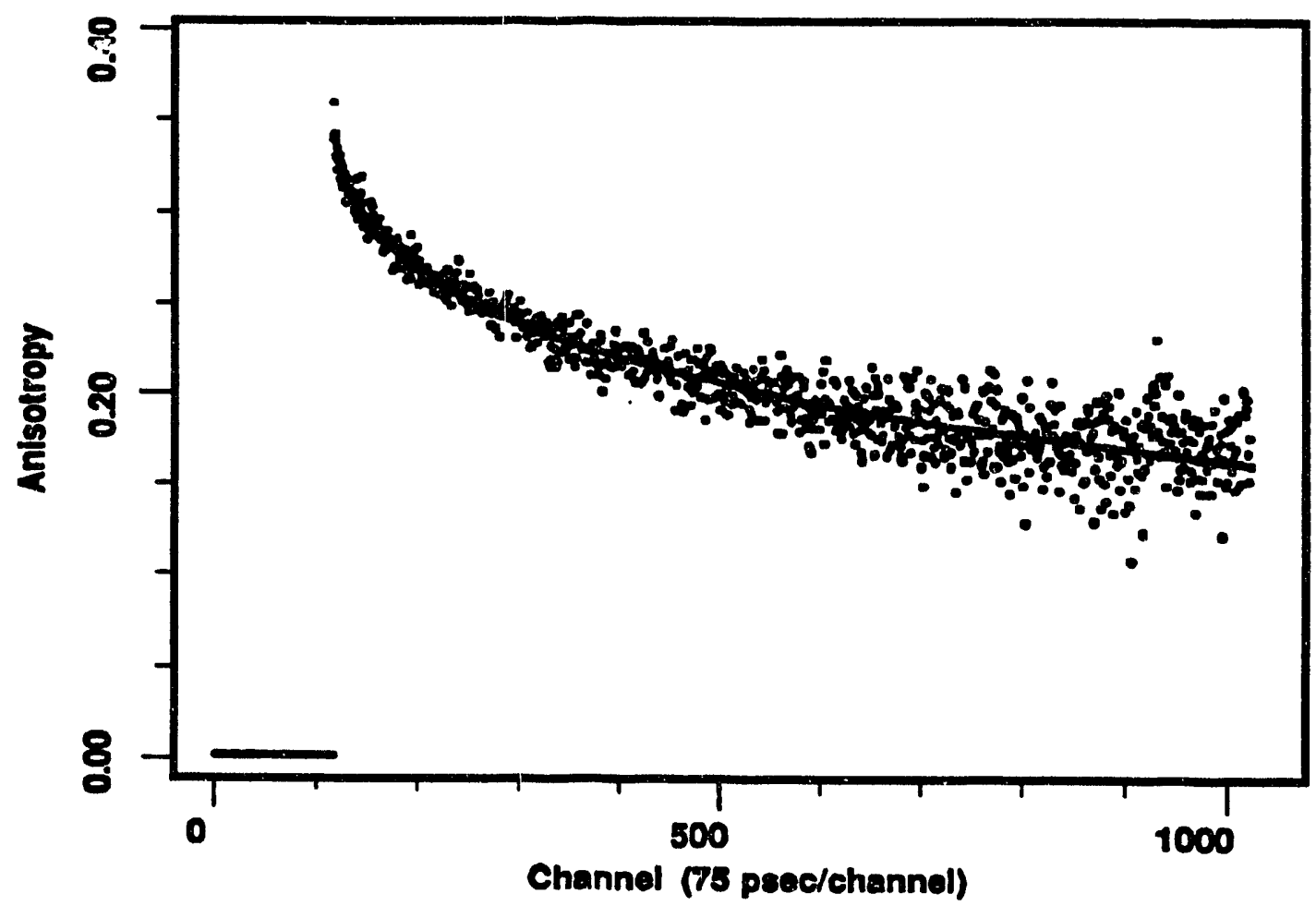

Fig 2.9B Lambda DNA in 24\% Sucrose

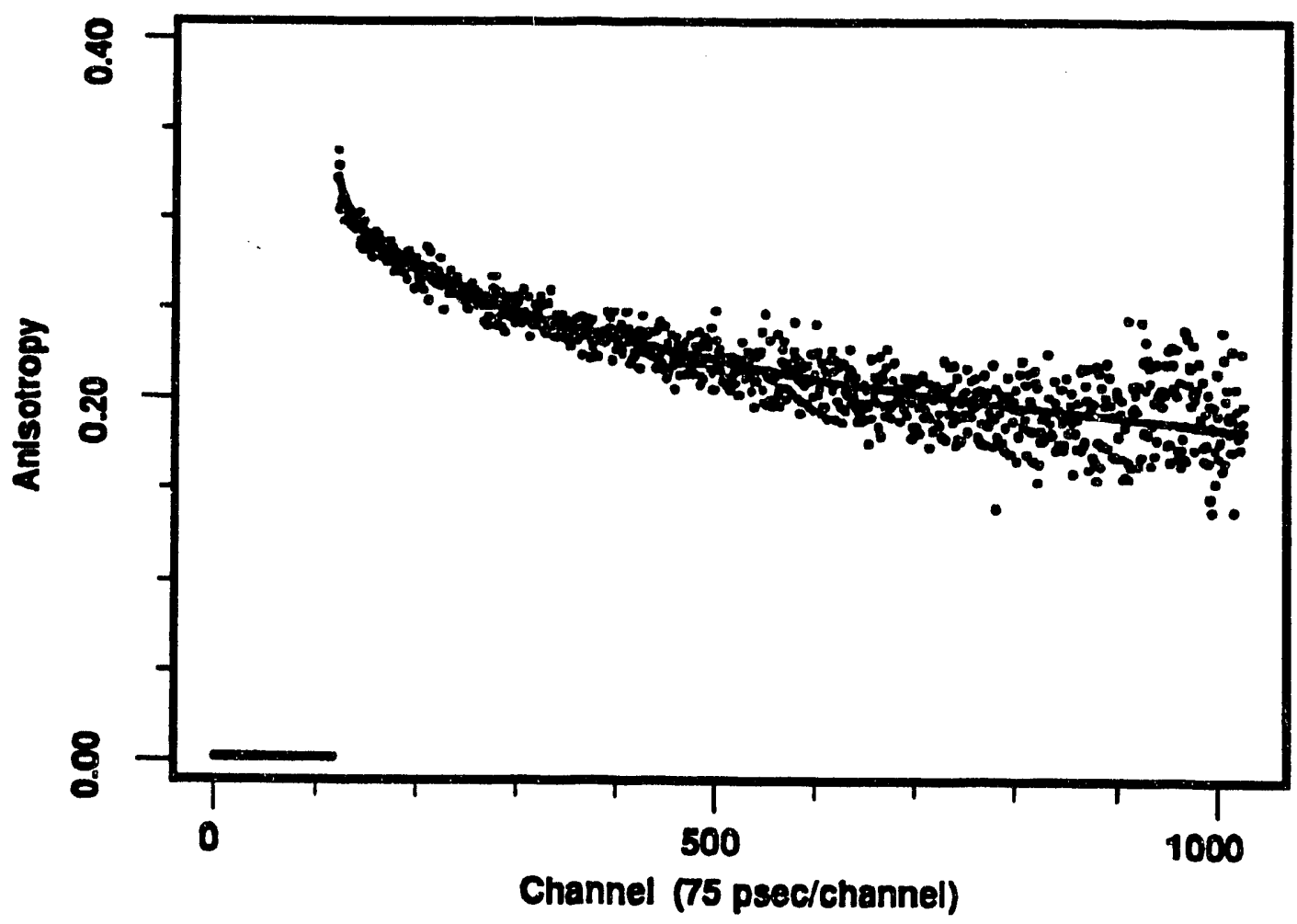


time scale of ethidium fluorescence, are not sufficient to completely randomize the fluorescence polarization from the sample. Twisting is thus a restricted motion compared to the overall tumbling modes. The mathematical expression of this fact is that twisting anisotropy decays as $\sqrt{t}$ and therefore changes only slowly at long times.

The fits of the data are plotted in the same graph in Fig 2.10. Several features are worth comment. First, the $0 \%$ sucrose data decay faster than do the $22 \%$ sucrose data. This is consistent with the idea that, in a medium of higher viscosity, the DNA is more immobile. Second, the final anisotropy of the $22 \%$ sucrose sample is significantly higher than that for $0 \%$ sucrose. The relative immobility of the DNA is thus reflected in the total anisotropy even at long times after the laser pulse. The difference between the $0 \%$ and $22 \%$ data sets is not large. But the fits for each of the intermediate sucrose concentrations fall between these extremes. This sucrose titration demonstrates that a change in viscosity of as little as $10 \%$ has a measurable effect on the FPA fit (Fig 2.8). Since the fit parameter $A$ has an identical functional dependence on $\eta_{\text {rel }}$ ar: - $C$, the sensitivity to differences in the torsion constant should be equal to the sensitivity to changes in viscosity. The effect of viscosity on the anisotropy decay is linear over a 2.5 -fold change in viscosity (Table 2.2 and Fig 2.8). Thus, the time-resolved FPA measurement should be sensitive to changes in the torsion elastic constant as small as $10 \%$. 
Fig 2.10 Fitted curves for 0 and $24 \%$ sucrose

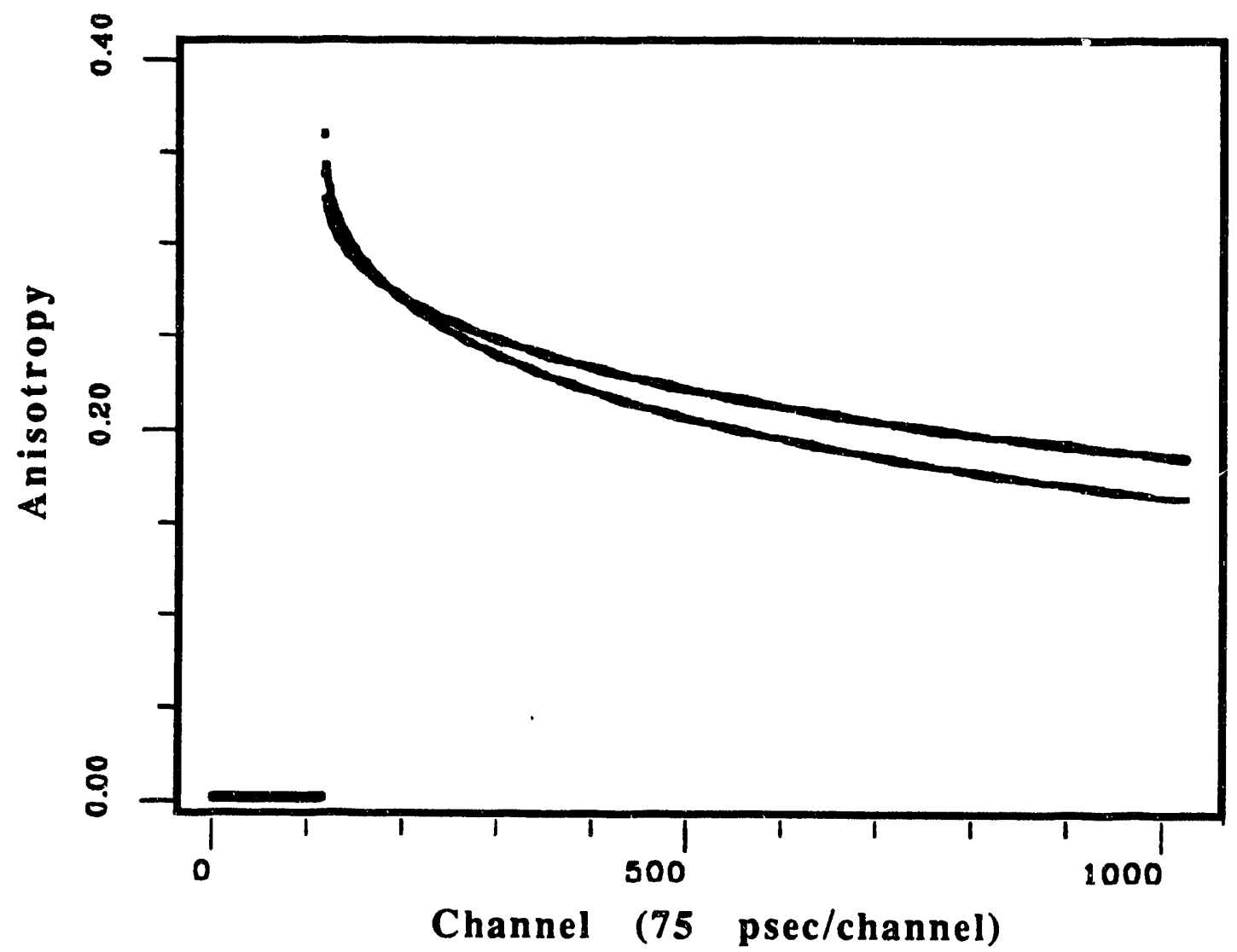

Fig 2.10. The two parameter fits to the anisotropy decay of lambda DNA in $0 \%$ and $24 \%$ sucrose. The lower curve is the decay for $0 \%$ sucrose and the upper curve is for $24 \%$ sucrose. Data were fit to the time-dependent anisotropy expression of Barkely and Zimm (1979) as described in the text. 
Torsional stiffness as a function of ionic strength. To determine whether ionic interactions play a role in torsional rigidity, the anisotropy decay was measured for linearized pBR322 as a function of ionic strength at three values ranging from $5 \mathrm{mM}$ to $325 \mathrm{mM}$ (Table 2.3). The torsion constant $\mathrm{C}$ decreases with increase in ionic strength, as predicted for a model in which ionic interactions between phosphates contribute to overall torsional stiffness. This observation is similar to experiments which demonstrate that DNA bends more easily with increases in salt.

Table 2.3. Torsion Constant of Linear pBR322 as a Function of Ionic Strength

\begin{tabular}{|c|c|}
\hline$\mu(\mathrm{mM})^{a}$ & $\mathrm{C} \times 10^{19}(\mathrm{erg} \cdot \mathrm{m})^{b}$ \\
\hline 5 & $2.14 \pm 0.04$ \\
\hline 175 & $1.91 \pm 0.06$ \\
\hline 325 & 1.60 \\
\hline $\begin{array}{l}\text { Ionic st } \\
\text { Torsion }\end{array}$ & \\
\hline
\end{tabular}

Eluorescence anisotropy measurements of DNA topoisomers. The torsion constant $\mathrm{C}$ was measured for a number of topoisomer samples spanning the range $\sigma=-0.123$ to +0.042 . In the high salt 
buffer used to prepare the relaxed DNA sample $(150 \mathrm{mM} \mathrm{KCl}, 50$ $\mathrm{mM}$ Tris $\cdot \mathrm{Cl}, \mathrm{pH} 8.0,1 \mathrm{mM}$ EDTA), $\mathrm{C}$ decreases monotonically with increased positive supercoiling of the samples (Table 2.4 and Fig

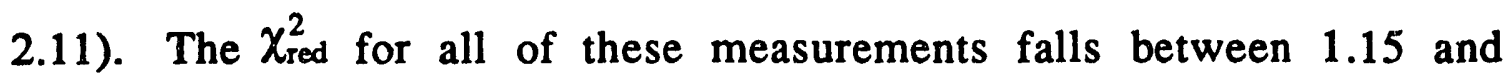
1.30. The data for linear DNA and for relaxed, covalently closed DNA are combined at $\sigma=0.0$ for the $150 \mathrm{mM} \mathrm{KCl}$ experiment. At this salt concentration, the helical repeat should be identical for relaxed and linear samples, and indeed, the overlap in the values for $C$ indicates that the DNAs show similar dynamic behavior.

Also plotted in Fig 2.11 are the torsion constant measurements for the topoisomer samples at low salt $(5 \mathrm{mM}$ Tris. $\mathrm{Cl}, 0.5 \mathrm{mM}$ EDTA, $\mathrm{pH}$ 8.0, Table 2.5). Changes in salt concentration have important effects on $L_{k_{0}}$. Reducing the ionic strength will a cause linear DNA to unwind since repulsive interactions between phosphates are less shielded by counterions from the solvent. A molecule cyclized under these conditions will have a smaller $L_{k_{0}}$ than a similar molecule ligated in higher salt. This means that $\Delta \mathrm{Lk}$ for the topoisomers prepared under the standard conditions becomes more negative as the ionic strength decreases. Although the data at low salt is plotted as a function of the measured $\sigma$, the true value for $\sigma$ under these solvent conditions is somewhat more negative. The difference $\Delta \sigma$ between various samples remains constant, however.

In low ionic strength buffer, the torsion constant of the samples between +0.042 and -0.069 increases compared to higher ionic strength conditions, consistent with the observation for linear DNA that $\mathrm{C}$ is inversely related to ionic strength. At higher negative superhelical densities $(\sigma=-0.096$ and -0.123$), C$ is indistinguishable 
Table 2.4. Data Summary for FPA Measurements in $150 \mathrm{mM} \mathrm{KCl}$, $50 \mathrm{mM}$ Tris.Cl, pH 8.0, 1 mM EDTA.

$\begin{array}{cccc}\text { Sample }^{a} & \mathbf{L}_{\mathbf{Q}}{ }^{b} & \mathbf{C}^{c} & \mathbf{\chi}_{\text {red }}^{2} \\ +0.042 \mathrm{a} & 0.3579 & 1.773 & 1.239 \\ +0.042 \mathrm{~b} & 0.3554 & 1.778 & 1.225 \\ +0.042 \mathrm{c} & 0.3602 & 1.728 & 1.264 \\ & & & \\ \text { lineara } & 0.3463 & 1.963 & 1.226 \\ \text { linearb } & 0.3397 & 1.895 & 1.162 \\ \text { linearc } & 0.3503 & 1.952 & 1.232 \\ \text { lineard } & 0.3564 & 1.933 & 1.175 \\ \text { lineare } & 0.3514 & 1.876 & 1.153 \\ \text { linearf } & 0.3518 & 1.816 & 1.240 \\ \text { relaxa } & 0.3388 & 1.806 & 1.211 \\ & & & \\ -0.025 \mathrm{a} & 0.2476 & 2.021 & 1.274 \\ -0.025 \mathrm{~b} & 0.3368 & 1.843 & 1.211 \\ & & & \\ -0.069 \mathrm{a} & 0.3535 & 2.175 & 1.302 \\ -0.069 \mathrm{~b} & 0.3453 & 2.023 & 1.229 \\ & & & \\ -0.096 \mathrm{a} & 0.3446 & 2.130 & 1.233 \\ -0.096 \mathrm{~b} & 0.3452 & 2.150 & 1.233 \\ & & & \\ -0.123 \mathrm{a} & 0.3381 & 2.353 & 1.245 \\ -0.123 \mathrm{~b} & 0.3445 & 2.307 & 1.224 \\ -0.123 \mathrm{c} & 0.3380 & 2.175 & 1.245\end{array}$

a Superhelix density of sample.

$b$ Initial anisotropy at $\mathrm{t}=0$.

c Torsion constant, x $10^{19}(\mathrm{erg} \cdot \mathrm{cm})$.

d Reduced chi-squared of fit to anisotropy decay. 
Fig 2.11. Torsion Constant of Topoisomer Samples

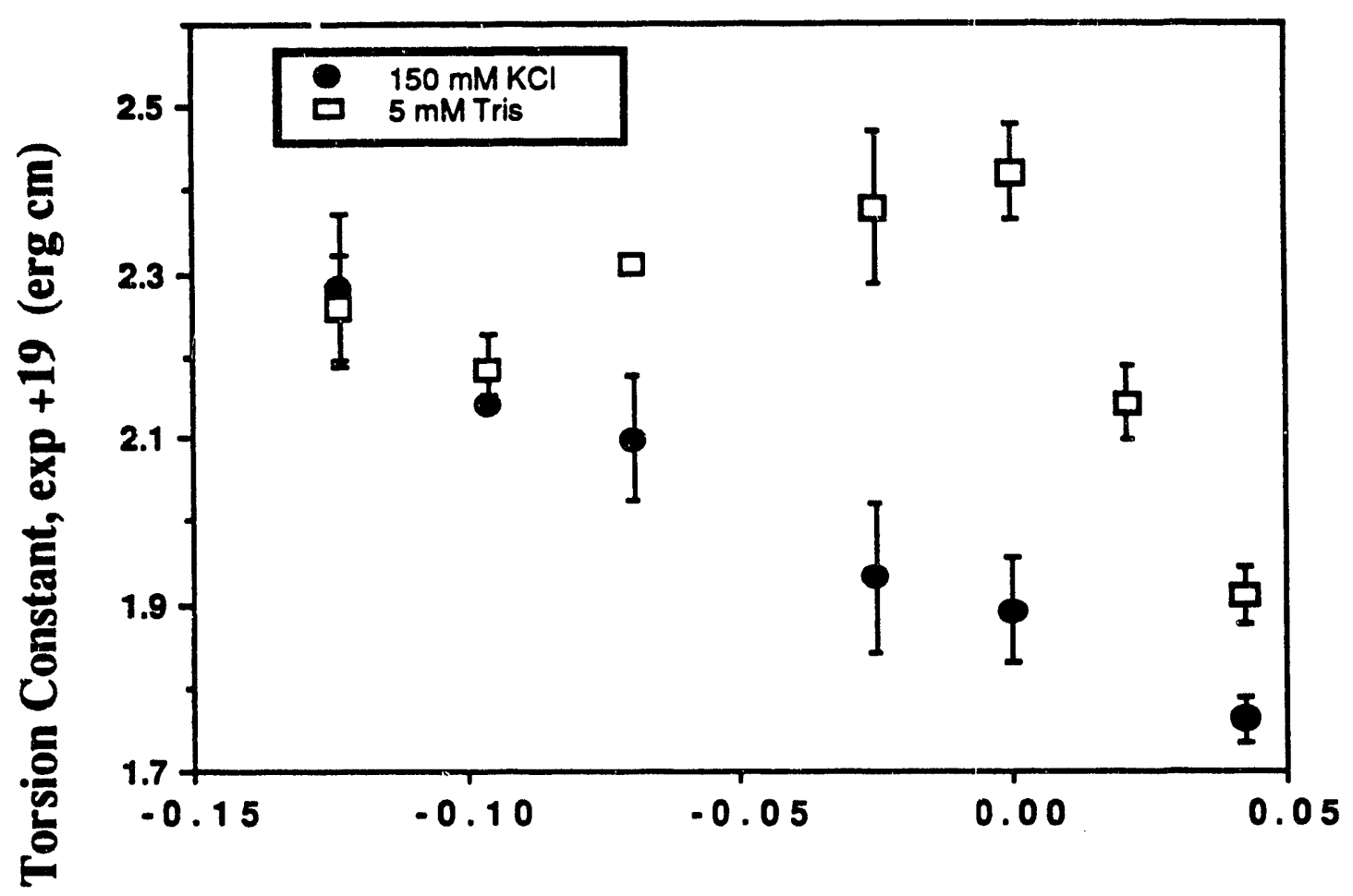

\section{superhelix \\ density}

Fig 2.11. Torsion constants of topoisomer samples in $150 \mathrm{mM} \mathrm{KCl}$ and $5 \mathrm{mM}$ Tris, $\mathrm{pH}$ 8.0. This plot represents the combined data from Tables 2.4 and 2.5. Error bars represent the standard deviation for $\mathbf{N} \geq 3$ or the spread between independent measurements for $\mathbf{N}=2$. 
Table 2.5. Data Summary for FPA Measurements in $5 \mathrm{mM}$ Tris. $\mathrm{Cl}$, pH 8.0, 0.5 mM EDTA.

\begin{tabular}{cccc} 
Sample $^{a}$ & $\mathfrak{L}_{0}{ }^{b}$ & $\mathbf{C}^{c}$ & $\boldsymbol{\chi}_{\text {red }}^{2}$ \\
\hline$+0.042 \mathrm{a}$ & 0.3580 & 1.950 & 1.215 \\
$+0.042 \mathrm{~b}$ & 0.3526 & 1.882 & 1.151 \\
$+0.042 \mathrm{c}$ & 0.3508 & 1.902 & 1.220 \\
& & & \\
linearb & 0.3535 & 2.152 & 1.186 \\
linearc & 0.3518 & 2.179 & 1.247 \\
lineard & 0.3390 & 2.092 & 1.155 \\
relaxa & 0.3528 & 2.479 & 1.196 \\
relaxb & 0.3489 & 2.362 & 1.255 \\
& & & \\
$-0.025 \mathrm{a}$ & 0.3464 & 2.468 & 1.229 \\
$-0.025 \mathrm{~b}$ & 0.3425 & 2.287 & 1.034 \\
& & & \\
$-0.069 \mathrm{a}$ & 0.3475 & 2.304 & 1.274 \\
$-0.069 \mathrm{~b}$ & 0.3478 & 2.316 & 1.275 \\
& & & \\
$-0.096 \mathrm{a}$ & 0.3435 & 2.224 & 1.236 \\
$-0.096 \mathrm{~b}$ & 0.3446 & 2.142 & 1.136 \\
& & & \\
$-0.123 \mathrm{a}$ & 0.3483 & 2.220 & 1.264 \\
$-0.123 \mathrm{~b}$ & 0.3424 & 2.323 & 1.180 \\
$-0.123 \mathrm{c}$ & 0.3417 & 2.301 & 1.117 \\
$-0.123 \mathrm{~d}$ & 0.3371 & 2.190 & 1.236
\end{tabular}

a Superhelix density of sample.

$b$ Initial anisotropy at $t=0$.

c Torsion constant, $\times 10^{19}$ (erg.cm).

d Reduced chi-squared of fit to anisotropy decay. 
from the values measured in $150 \mathrm{mM} \mathrm{KCl}$. Possible reasons for thiseffect on the helical repeat of DNA (Anderson and Bauer, 1978) are discussed below. An additional interesting result is that, at low ionic strength, $\mathrm{C}$ is relatively constant for all of the negatively supercoiled samples.

At low salt, there is a striking transition between relaxed DNA and overwound DNA (Fig 2.11). Fcr the three measurements of the positively supercoiled samples, $C$ is tightly clustered around an average value of $C=1.91 \times 10^{-19} \mathrm{erg} \cdot \mathrm{cm}$ (Table 2.5). This value is distinctly different from the two measurements of relaxed DNA, C = $2.42 \times 10^{-19} \mathrm{erg} \cdot \mathrm{cm}$.

An additional observation supports the idea that the transition between positive and negative supercoiling results in a marked difference in torsion constant at low salt. The data for three measurements of linear DNA at low salt are plotted in Fig 2.11. Although the relative position along the $\mathrm{x}$-axis of these points is uncertain due to the unknown change in helical repeat at low salt, it is certain that the direction of offset along the $x$-axis, relative to the covalently closed samples, is to the right. The torsion constant for the linear sample in low salt is midway between that for the relaxed sample (which is actually underwound at low ionic strength) and the positive sample, supporting the idea that there is a sharp transition in the torsion constant between positive to negative superhelix densities at low ionic strength. 


\section{Discussion}

Comparison of torsion constant for linear DNA with results from the literature. The torsion elastic constant of DNA has been measured a number of times by different investigators using both the static ligation method and dynamic spectroscopic techniques (Table 2.5). The value of the torsion constant measured in the experiments described in this chapter for linear pBR322 DNA in $150 \mathrm{mM} \mathrm{KCl}$ is consistent with the many values catalogued below, $C=1.90 \pm 0.06$ $x 10^{-19} \mathrm{erg} \cdot \mathrm{cm}$. This value has been obtained using the assumption that the persistence length $P=510 \AA$ (Millar et al., 1982) and that the flexural rigidity $\mathrm{EI}=\mathrm{k}_{\mathrm{b}} \mathrm{TP}=2.15 \times 10^{-19} \mathrm{erg} \cdot \mathrm{cm}$ (Barkley and Zimm, 1979).

Measurements of ring closure probability were originally performed by Shore and Baldwin (1983a,b) who used classical thermodynamics to calculate $C$. Their estimate of $C=2.4 \times 10^{-19}$ erg. $\mathrm{cm}$ was in reasonable agreement with previous spectroscopic measurements (Millar et al., 1982; Thomas and Schurr, 1983). Horowitz and Wang performed similar experiments resulting in an estimate of $C=3.0 \times 10^{-19} \mathrm{erg} \cdot \mathrm{cm}$ (Horowitz and Wang, 1984). A number of groups have analyzed the Shore and Baldwin data using Monte Carlo simulations, fitting the data to a torsion constant in the range of $\mathrm{C}=2.3-3.1 \times 10^{-19} \mathrm{erg} \cdot \mathrm{cm}$ (Frank-Kamenetskii et al., 1985; Hagerman and Ramadevi, 1990; Shimada and Yamakawa, 1984). The highest value of $3.8 \times 10^{-19}$ for the Shore and Baldwin data set, obtained by Levene and Crothers (1986), has recently been challenged based on their criterion for successful simulated ring closure (Hagerman, 1990). Levene and Crothers scored chains as 
closed if the end-to-end separation was less than $20 \%$ of the persistence length $\mathrm{P}$. For a 250 bp molecule, such as that studied by Shore and Baldwin, and with $\mathrm{P}=500 \AA, 0.2 \mathrm{P}$ corresponds to approximately $12 \%$ of the contour length of the molecule (Hagerman, 1990). Use of this generous criterion for ring closure will lead to an overestimate of $\mathrm{C}$ since even very stiff chains can cyclize readily. A recent measurement of $\mathrm{C}$ using the cyclization methodology for a different DNA sequence has given a best fit value of $C=2.0 \times 10^{-19}$ (Taylor and Hagerman, 1990). Thus, measurements based on the static ligation method yield results in the range of $2.0-3.1 \times 10^{-19}$ erg.cm.

Time-resolved spectroscopic measurements have generally yielded values for $\mathrm{C}$ which are somewhat lower than those obtained with the static techniques. Schurr and collaborators have made numerous measurements, but their analysis, unlike that presented in this chapter, ignores the contribution of bending to fluorescence depolariztion (Fujimoto and Schurr, 1990; Shibata et al., 1984; Taylor and Hagerman, 1990; Thomas et al., 1980; Thomas and Schurr, 1983; Wu et al., 1988). Their values of the torsion constant of linear DNA all fall near $C=1.3$ to $1.4 \times 10^{-19} \mathrm{erg} \cdot \mathrm{cm}$. Applying what they believe to be the best correction for bending revises their measurement upwards to $C=1.9 \times 10^{-19} \mathrm{erg} \cdot \mathrm{cm}$. This estimate is in good agreement with the results of Millar et al. (1982) who measured $C=1.95 \pm 0.18 \times 10^{-19} \mathrm{erg} \cdot \mathrm{cm}$ for supercoiled $\mathrm{pBR} 322$ of unspecified superhelix density and $1.43 \pm 0.11 \times 10^{-19} \mathrm{erg} \cdot \mathrm{cm}$ for calf thymus DNA, both in $150 \mathrm{mM} \mathrm{NaCl}, 100 \mathrm{mM}$ Tris.Cl, $\mathrm{pH}$ 7.7. In these experiments, the calf thymus DNA was commercially obtained 
and not subjected to further purification. A different method, timeresolved EPR spectroscopy of intercalated, spin-labeled probes yielded a value of $C=1.2 \times 10^{-19} \mathrm{erg} \cdot \mathrm{cm}$ using a theory which neglects bending (Robinson et al., 1980).

The differences between the results from static and dynamic techniques merit consideration. The dynamic measurements suffer from the poorly understood contribution of DNA bending to depolarization, which could lead to an underestimate of the torsion elastic constant. Fujimoto and Schurr (1990), estimating a maximal contribution of bending to depolarization, determine an upper bounds limit for the torsion constant derived from their FPA data, C $=2.55 \times 10^{-19} \mathrm{erg} \cdot \mathrm{cm}$. This upper value for $\mathrm{C}$ is squarely in the middle of estimates made by the ligation technique. Fujimoto and Schurr stress, however, that even a lower bound estimate of $\mathrm{C}$, which ignores bending, should faithfully monitor any changes in the actual torsion constant.

The static measurements have significant pitfalls as well. Most of the estimates have been based on a single data set, that of Shore and Baldwin (1983a,b). Nevertheless, analysis of a single data set gives rise to a range of estimates for $\mathrm{C}$ implying that the assumptions in a particular method of calculation strongly influence the result. The Monte Carlo method simultaneously fits a number of parameters, including pitch, $\mathrm{P}$, helical repeat, $\mathrm{h}$, and torsion constant, C. A decrease in the estimated value of one parameter can be compensated for by an increase in another to fit the data. Choosing a particularly low value for the persistence length $P$ will increase the fitted value of the torsion constant C. This fitting procedure and 
the criterion used to evaluate the goodness of fit can also influence the resulting estimate of the torsion constant.

Variation of torsion constant with superhelicity. The experiments described here demonstrate that $\mathrm{C}$ varies as a function of the superhelicity of DNA at physiological salt concentrations. This is a result that cannot be obtained by static methods, since the product of ligation is by definition a set of topoisomers centered around $\mathbf{L k}_{\mathbf{0}}$. Millar et al. (1982) suggested a possible dependence of $\mathrm{C}$ on superhelix density, but their observation was based on a single comparison between calf thymus DNA and supercoiled pBR322. Nevertheless, the magnitude of the difference in $\mathrm{C}$ between their two samples is reasonably consistent with that which has been reported here (Fig 2.11).

Other attempts to measure $\mathrm{C}$ as a function of superhelix density, by Schurr and colleagues, have either observed no difference (Wu et al., 1988) or have seen drastic, time-dependent transitions in $\mathrm{C}$ over the course of several weeks for a single sample (Shibata et al., 1984; Song et al., 1990). The former work examined pBR322 which was linearized or which was supercoiled to $\sigma=-0.048$ or -0.083 . In $0.1 \mathrm{M} \mathrm{NaCl}, \mathrm{Wu}$ et al. measured a single torsion constant for all three samples of $2.3 \pm 0.2 \times 10^{-19} \mathrm{erg} \cdot \mathrm{cm}$ (corrected for bending using their best estimate) (Wu et al., 1988). Comparing these results to those presented here, it becomes clear that measurement over a wider range of superhelical densities establishes the trend in torsion constant which might be missed by looking at a more limited set of samples. For instance, for the 
samples with superhelical densities $\sigma=0.0,-0.069$, and $-0.096, C$ is here measured to be $1.90 \pm 0.06,2.01 \pm 0.10$, and $2.14 \pm 0.01$, respectively (Table 2.4). This variation in torsion constant is confirmed by measurement of further extremes in superhelical density, but might be missed without additional measurements. In fact, it is significant that $C$ has now been measured for positively supercoiled DNA, since overwound DNA may be more important in vivo than previously suspected (Liu and Wang, 1987; Rahmouni and Wells, 1989). For the positively supercoiled DNA, the torsion constant is even smaller, $C=1.75 \times 10^{-19} \mathrm{erg} \mathrm{cm}$, confirming the trend as a function of superhelicity. (It is worth noting that in a very recent paper, Schurr and collaborators have reversed their statement that the torsion constant does not change as a function of superhelix density; they maintain presently that there is an increase in $C$ from relaxed to negatively supercoiled samples (Song et al., 1990).)

The data presented at low ionic strength are surprising in some respects, but in general, they confirm the idea that DNA stiffness, both bending and torsional, is a function of the ionic strength of the solvent. Previously, Millar et al. observed this dependence for linear DNA over a much larger range of ionic strengths (Millar et al., 1982). At the most negative superhelix densities $(\sigma=-0.096$ and -0.123 ), $C$ is unaffected by changes in ionic strength (Fig 2.11) The constant value of $\mathrm{C}$ at high negative superhelix densities may have a simple explanation. However, it seems more likely that this phenomenon is the fortuitous result of several complex and of fsetting interactions. 
The sharp transition between positively and negatively supercoiled samples at low salt is striking, especially in view of the smooth trend observed at high salt (Fig 2.11). It is difficult to speculate on the origin of this phenomenon except to say that it may reflect some different global conformation adopted by overwound DNA compared to underwound DNA at low ionic strength. It is noteworthy that under both high and low salt conditions, positively supercoiled DNA is torsionally less stiff than is negatively supercoiled or relaxed DNA. This flexibility may indeed allow it to assume some different secondary structure under low salt which is not accessible to underwound DNA.

Potential effects on the biological function of DNA. The flexibility of DNA is important in many of its biological roles, including packaging in viral particles (Widom and Baldwin, 1983), loop formation by regulatory proteins (Dunn et al., 1984; Kramer et al., 1987), sitespecific recombination (Nash, 1990), organization into chromatin (Klug et al., 1980), and curvature induced by site-specific DNA binding proteins (Gartenberg and Crothers, 1988). The finding that torsional flexibility varies as a function of supercoiling may have important implications for some of these functions. The discussion presented here focuses on the implications for DNA loop formation by a regulatory protein.

The torsion constant $\mathrm{C}$, a length independent quantity, is related to the molar twisting coefficient $\mathrm{K}_{\mathrm{Tw}}$, for a particular molecule, by the relationship (Shore and Baldwin, 1983a):

$$
\mathrm{C}=\left(\mathrm{NK}_{\mathrm{Tw}} / \mathrm{RT}\right)\left(\mathrm{k}_{\mathrm{b}} \mathrm{T} l / 2 \Gamma \mathrm{i}^{2}\right)
$$


where $\mathbf{N}$ is the number of base pairs, $R$ is the gas constant, $T$ is the temperature in Kelvins, $\mathrm{k}_{\mathrm{b}}$ is Boltzmann's constant, and $l$ is the distance between base pairs $\left(3.4 \times 10^{-8} \mathrm{~cm}\right)$. The molar twisting coefficient, $K_{T w}$, relates the fractional twist of a particular molecule, $\delta \mathrm{Tw}$, to the free energy of twisting (Shore and Baldwin, 1983a):

$$
\Delta \mathrm{G}_{\mathrm{Tw}}=\mathrm{K}_{\mathrm{Tw}}(\delta \mathrm{Tw})^{2}
$$

These equations allow one to predict the binding free energy required when a protein must twist the DNA in order to bind.

Consider a protein such as the lac repressor which recognizes two sites separated by 500 base pairs and loops out the intervening DNA upon complex formation. If the two sites are located on opposite faces of the DNA, then $(\delta \mathrm{Tw})^{2}=0.25$ in order to align the binding sequences. If the superhelical stress in the DNA varies, then the binding energy required to overcome this twisting will also change. Such a model is not unreasonable for an upstream regulatory region of DNA, given that current evidence suggests that the superhelical density upstream from a gene can vary as a result of transcription (Liu and Wang, 1987; Rahmouni and Wells, 1989; Wu et al., 1988). For DNA in the relaxed state, $\sigma=0.0$ and $C=1.9 \mathrm{x}$ $10^{-19} \mathrm{erg} \cdot \mathrm{cm}$, whereas for highly underwound DNA, $\sigma=-0.10$ and $\mathrm{C}=$ $2.2 \times 10^{-19} \mathrm{erg} \cdot \mathrm{cm}$. This results in $\Delta \mathrm{G}_{\mathrm{Tw}}=+2.97 \mathrm{~kJ} / \mathrm{mol}$ for the relaxed case, and $\Delta G_{T w}=+3.44 \mathrm{~kJ} / \mathrm{mol}$ for the underwound case. The difference is $\Delta \Delta G_{T w}=-0.47 \mathrm{~kJ} / \mathrm{mol}$, going from the underwound DNA to the relaxed case. Converting this free energy difference to an equilibrium constant, it becomes apparent that the change in torsional stiffness of DNA has only a small effect on which DNA, relaxed or underwound, is more likely to form a loop: 


$$
\mathrm{K}_{\mathrm{eq}}=\exp \left(\frac{\Delta \Delta \mathrm{G}_{\mathrm{Tw}}}{\mathrm{RT}}\right)=1.20
$$

It is striking, in fact, that the absolute binding energy required for a protein to twist a 500 bp DNA by one-half turn is so large, in the range of 3.0 to $3.5 \mathrm{~kJ} / \mathrm{mol}$.

The preceding discussion indicates that it is unlikely that changes in torsion constant as a function of supercoiling lead. directly to biological regulation. The magnitude of free energy required to twist DNA is large, irrespective of the initial superhelicity of the molecule. However, an indirect means of regulation due to changes in torsional stiffness can be imagined. It is possible that cells may induce specific proteins which can alter the torsional flexibility of DNA. This has already been shown to be the case for bending flexibility of DNA in $E$. coli where it is widely recognized that interaction of $\mathrm{HU}$ protein with DNA dramatically decreases its stiffness (Broyles and Pettijohn, 1986; Dixon and Kornberg, 1984; Flashner and Gralla, 1988; Hodges-Garcia et al., 1989; Johnson et al., 1986; Pettijohn, 1988). It is possible that similar proteins could perform this function to decrease torsional stiffness. Regulated expression of these proteins, or regulation of their binding to DNA, could result in altered torsional stiffness and lead to changes in gene expression.

The dynamics of DNA twisting. DNA responds in a non-linear fashion to the torsional stress of supercoiling. To explore the consequence of this fact on the motion of individual base pairs, I would like to develop a simple physical analogy between the 
dynamic behavior of a DNA base pair and that of a torsional pendulum. This analogy provides insight into the molecular dynamics of DNA twisting. In particular the thermal fluctuations in the twist angle between base pairs is shown to be large compared to the average deviation in twist angle induced by supercoiling of the double helix. Furthermore, the breadth of the thermal oscillations in twist angle means that the base pair motions sample a significant portion of the anharmonic twisting potential surface. Finally, the initial assumption of the FPA theory, which presumes that the DNA base pairs can be described as simple harmonic oscillators, is evaluated in light of the data.

An ideal torsional pendulum with torsion constant $\kappa$ is illustrated in Fig 2.12A. The pendulum is of molecular dimensions and is surrounded by a Brownian heat bath, so it has an average potential energy of $1 / 2 k_{b} T$. An arrow is painted onto the disk sothat an observer can determine the twist angle relative to some fixed reference point. The equation of motion for the system is $\alpha=$ $\alpha_{m} \cos \omega t+\alpha_{0}$, where $\alpha_{0}$ is the equilibrium twist angle for the relaxed pendulum, $\alpha_{m}$ is the maximum amplitude of the motion, determined by the temperature of the heat bath, and $\omega$ is the angular frequency, equal to $\sqrt{\frac{K}{I}}$, where $I$ is the rotational inertia of the pendulum.

At the overhead attachment point, the ribbon is fixed to an adjustable rachet which allows the observer to change the equilibrium twist angle. The pendulum is set to some new equilibrium angle because the ratchet applies a torque on the rivbon (Fig 2.13B). Equilibrium is established when the restoring torque 
Fig 2.12 Twist energy of an ideal torsional pendulum
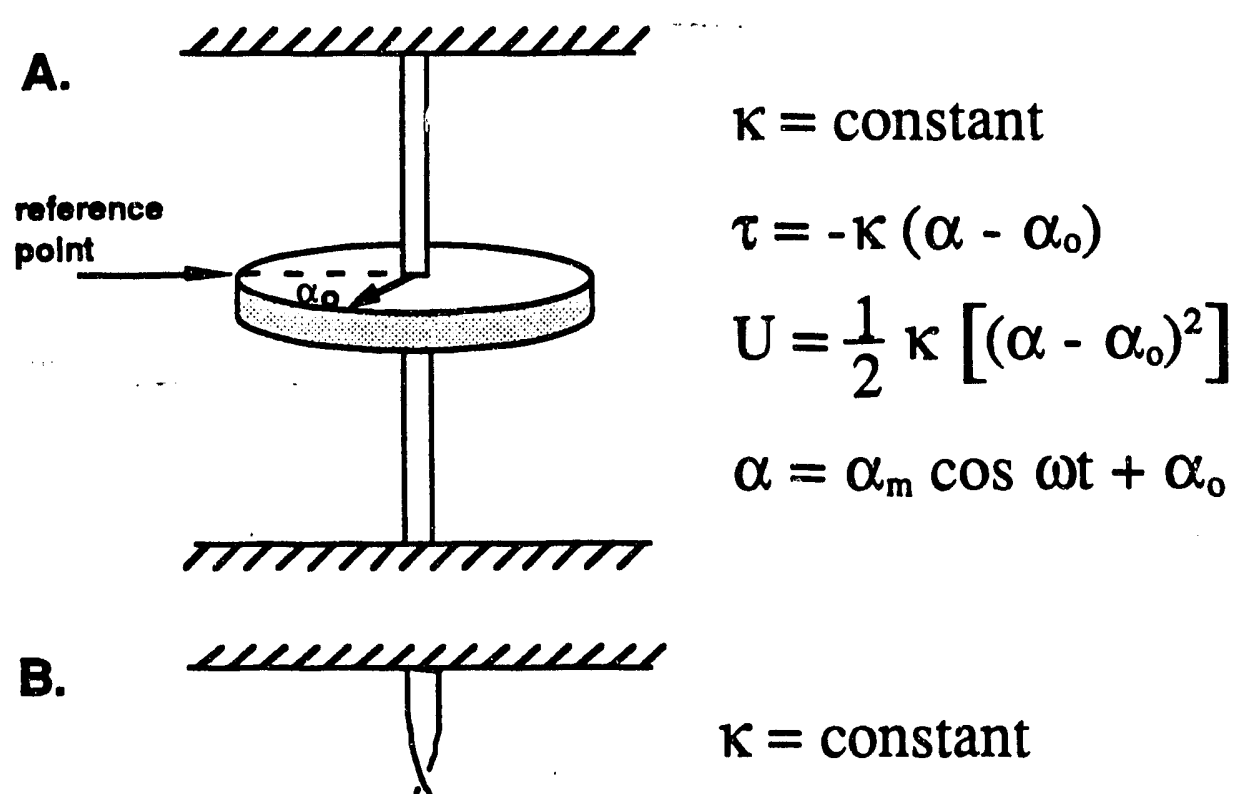

$\kappa=$ constant

reference

point

$$
\tau=-\kappa\left(\alpha-\alpha_{0}^{\prime}\right)
$$$$
U=\frac{1}{2} \kappa\left[\left(\alpha-\alpha_{0}^{\prime}\right)^{2}+\left(\alpha_{0}^{\prime}-\alpha_{0}\right)^{2}\right]
$$

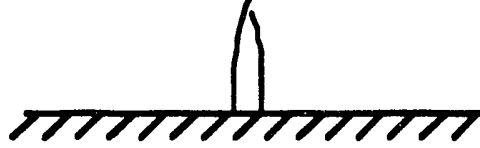

$\alpha=\alpha_{\mathrm{m}} \cos \omega t+\alpha_{\mathrm{o}}^{\prime}$

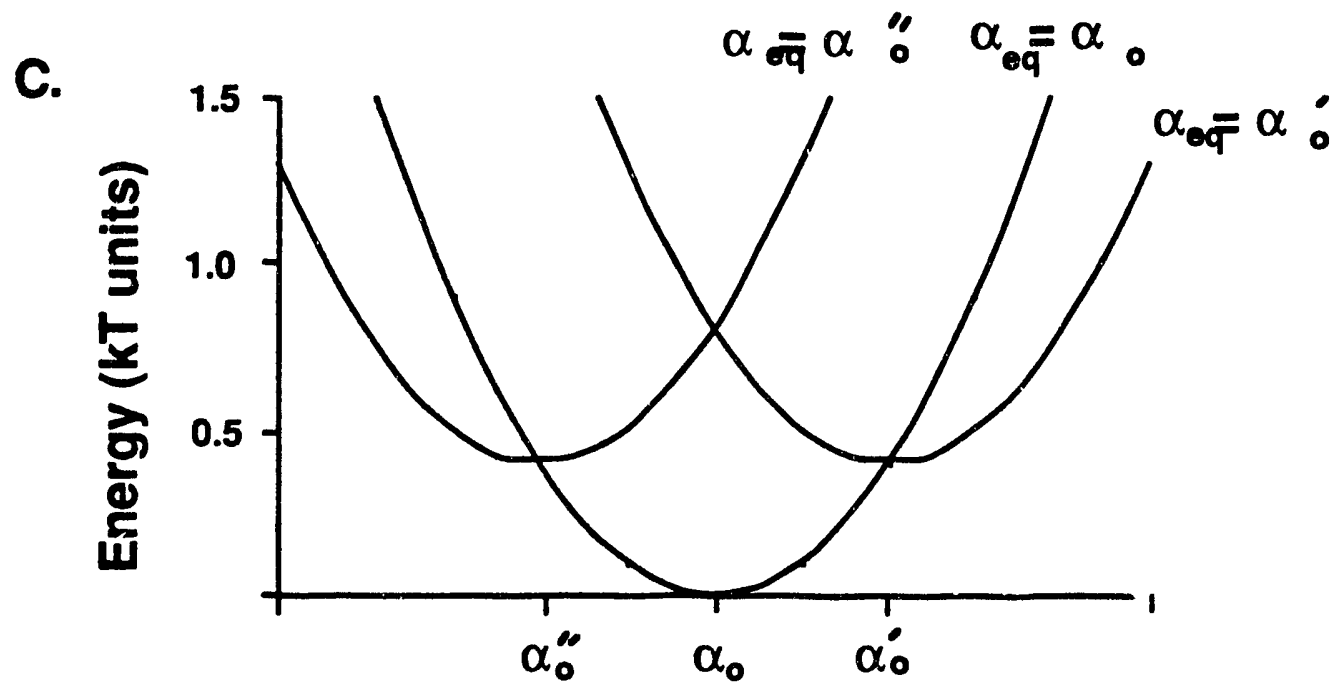

Twist angle 
from the ribbon is equal in magnitude to the torque of the ratchet. Since the pendulum is ideal, the torsion constant $k$ is the same for any amount of over- or underwinding. The new equation of motion is therefore $\alpha=\alpha_{m} \cos \omega t+\alpha_{0}^{\prime}$ where $\alpha_{0}^{\prime}$ is the new equilibrium twist angle. The angular frequency $\omega$ is unchanged since it does not depend on the equilibrium angle.

Although twisting the ribbon does not affect the dynamics of the pendulum, it does alter the potential energy of the system. The torque applied by the ratchet alters the equilibrium twist angle $\alpha_{\text {eq }}$ of the pendulum, and the potential energy varies with the square of this angular displacement. The twisted pendulum is displaced to higher energy compared to its natural equilibrium position in the absence of an external torque. Potential energy versus twist angle is plotted for three different values of $\alpha_{\mathrm{eq}}$ in Fig 2.12C. The minima in energy for states where the ratchet applies an external torque sit on the curve for $\alpha_{e q}=\alpha_{0}$ because the displacement energy relative to the natural equilibrium state is given by

$$
\mathrm{U}=\frac{K}{2}\left(\alpha_{\mathrm{eq}}-\alpha_{0}\right)^{2}
$$

The shape of each potential well is determined only by $k$, and therefore each curve is identical except for this offset energy. The amplitude of the thermal oscillation is given by the breadth of the energy well at $U=1 / 2 \mathrm{k}_{\mathrm{b}} \mathrm{T}$ :

$$
\left\langle\Delta \alpha_{\mathrm{rms}}=\left(\frac{\mathrm{k}_{\mathrm{b}} \mathrm{T}}{\mathrm{K}}\right)^{\frac{1}{2}}\right.
$$

A stiffer torsional pendulum, one with a larger torsion constant $x$, would have a steeper potential surface and thus a more limited range of thermal motion. 
Next we make the pendulum non-ideal by allowing $x$ to vary with angular displacement. The simplest situation, which corresponds to the supercoiling data of Fig 2.11 , is that $\mathrm{K}$ varies linearly with $\alpha: k=m \alpha+b$. The solution of the equation of motion in this case is much more complicated than for the ideal pendulum. The potential energy function is still quite simple, however, and provides significant physical insight into the thermally accessible states of the pendulum (Fig 2.13). Allowing $\mathrm{K}$ to vary linearly with $\alpha$ adds a cubic term to the spring energy (Fig 2.13A and B). The slope $m$ determines the degree of anharmonicity in the pendulum. When $m$ is negative, as is the case for DNA, then positive displacements require less energy than negative displacements of the same magnitude. This is shown in Fig $2.13 \mathrm{C}$ where the energy surface rises more steeply for negative than for positive deviations from the equilibrium angle $\alpha_{0}$. Oscillations about any new equilibrium angle $\alpha_{\text {eq }}$ sample a different anharmonic potential surface. For $\alpha_{e q}>\alpha_{0}$, the potential surface is broadened. At a given temperature, more twisted states are accesible to the dynamic system. For $\alpha_{e q}<\alpha_{0}$, the potential surface rises more steeply and fewer states are accessible. The motion of the pendulum can no longer be described as a simple harmonic function.

How can we relate this analogy to DNA? Each base pair can be modeled as a torsional pendulum. Measuring the angle between the reference point and the arrow on the torsional pendulum is equivalent to knowing the twist angle between adjacent DNA base pairs. Supercoiling of the DNA applies an external torque on the helix, just as the ratchet applies a torque on the torsional pendulum 
Fig 2.13. Twist energy of a non-ideal pendulum
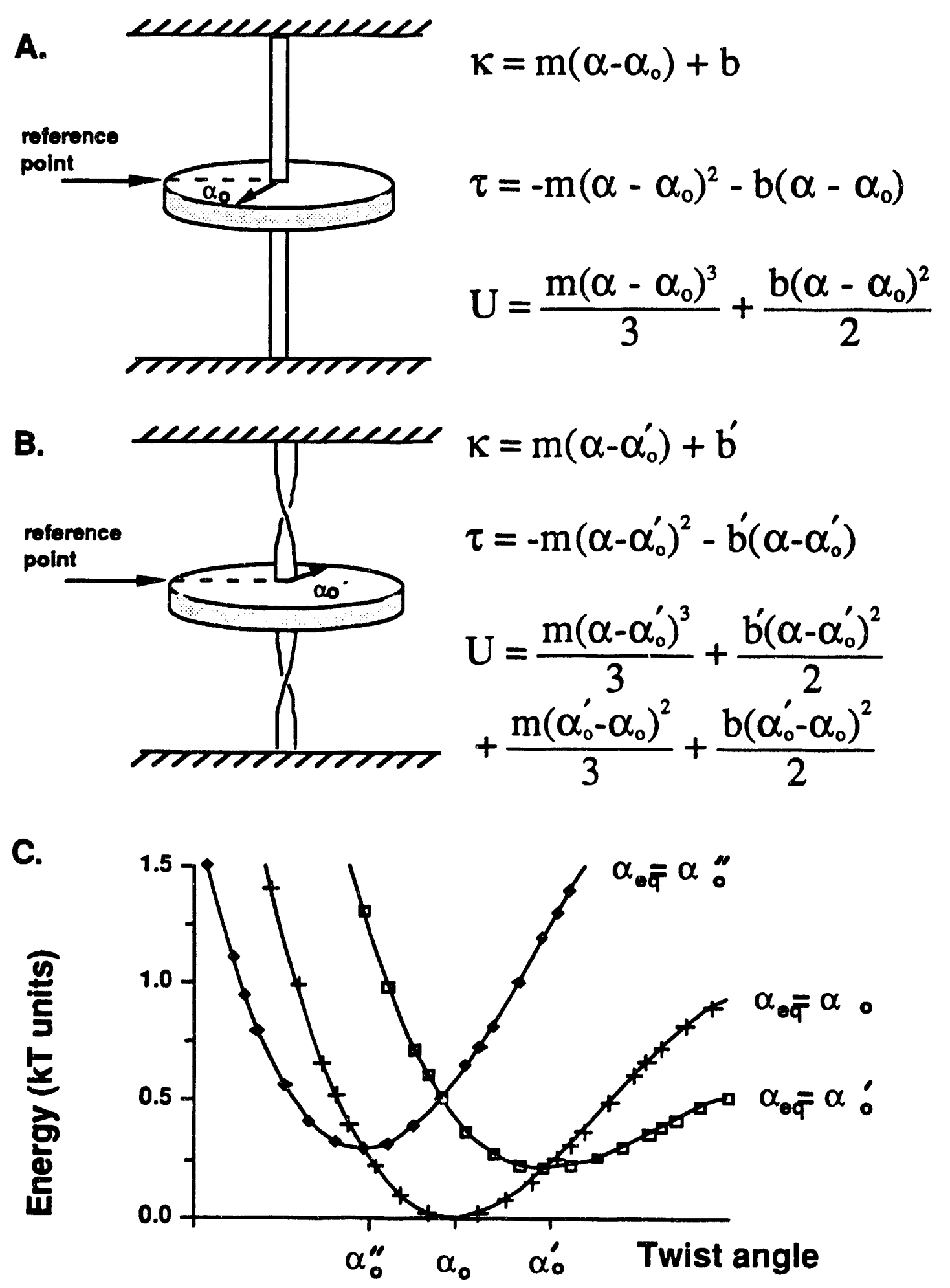
in our example. The supercoiling torque changes the equilibrium twist angle between base pairs. Since DNA is a non-ideal spring, this variation in twist angle is manifested as a change in the torsion constant of the system. The potential energy as a function of twist angle is therefore asymmetric, and its shape depends on the position of the equilibrium twist angle $\alpha_{\text {eq }}$. Qualitatively, the potential energy surface should look similar to that for the torsional pendulum depicted in Fig 2.13C. The accessible states for thermal fluctuations in twist angle will vary with over- and underwinding of the helix.

It is important to note that the thermal fluctuations in twist angle are large compared to the changes in equilibrium twist angle induced by supercoiling. With an average thermal energy of $1 / 2$ $k_{b} T$ per base pair, the thermal deviation in twist angle $\phi$ is given by

$$
\langle\Delta \phi\rangle_{\mathrm{rms}}=\left(\frac{\mathrm{h} \mathrm{k}_{\mathrm{b}} \mathrm{T}}{\mathrm{C}}\right)^{\frac{1}{2}}
$$

where $h$ is the rise per base pair, taken as $3.4 \AA$. The value of $\langle\Delta \phi\rangle_{\mathrm{rms}}$

for relaxed DNA is $5^{\circ}$. The thermal oscillation in $T w$ can also be estimated from this local base pair deviation by considering a deviation in $T w$ to be a random walk of step length $5^{\circ}$ and step number $\mathrm{N}$ equal to the number of base pairs in the molecule. For pBR322 ( $\mathrm{N}=4363 \mathrm{bp})$, the average deviation in $\mathrm{Tw}$ due to thermal osciallation of the base pair twist angle is $\pm\left(\sqrt{\mathrm{N}} \times 5^{\circ}\right) / 360^{\circ}$ or \pm 0.9 .

There is an apparent contradiction between the local flexibility of a DNA base pair, which undergoes large amplitude twisting motions, and the resistance of the helix to torsional deformation. 
These differences can be easily reconciled. Thermal fluctuations are random and uncorrelated. Large deviations in twist angle at one base pair are compensated for by displacements of opposite sign at other base pairs. The thermal fluctuations in $T w$ are therefore small, as demonstrated above. A concerted change in Tw due to supercoiling, on the other hand, results in a constant average angular displacement of each base pair. The energy required for this process is summed over all of the base pairs in the molecule. Since the potential energy in base pair twist varies as the square of the angular displacement, the total energy required to alter Tw rises sharply as the equilibrium twist angle is varied.

There are important differences between the simple torsional pendulum and DNA. For the torsional pendulum, all of the external torque goes into twisting potential energy. For DNA, some of the change in Lk, which can all be put in as local twisting by a topoisomerase enzyme, ends up as writhing of the supercoiled structure. The ability of DNA to bend allows it to distribute the deformation of supercoiling over several different internal modes, including bending and twisting, and thus minimize its total energy. At a maximum, a change in linking number of $\Delta \mathrm{Lk}$ would change the equilibrium twist angle between base pairs by $2 \pi \Delta \mathrm{Lk} / \mathrm{N}$ radians. By partitioning some of $\Delta \mathrm{Lk}$ into bending, the actual change in the equilibrium twist angle will be much less than this, and the total energy of the molecule will be considerably lower. This complication means that the torsion constant vs. superhelix density data in Fig 2.11 cannot be automatically translated to a similar plot of torsion constant vs. base pair twist angle. Complete elaboration of 
the dynamics of twisting of a DNA base pair depends on an understanding of how the equilibrium twist angle $\alpha_{\text {eq }}$ varies with Lk. This, in turn, means that one must know how changes in Lk are apportioned between $\mathrm{Tw}$ and $\mathrm{Wr}$.

Klenin et al., using a Monte Carlo simulation technique, have recently calculated that $\Delta \mathrm{Lk}$ is partitioned $30 \%$ into $\Delta \mathrm{Tw}$ and $70 \%$ into $\mathrm{Wr}$ (A. V. Vologodskii, personal communication). These authors used a torsion stiffness of $\mathrm{C}=3.0 \times 10^{-19} \mathrm{erg} \mathrm{cm}$ and a persistence length of $P=500 \AA$. The greatest uncertainty in their calculations is the helix diameter $d$, for which they used a range of values. They assumed, significantly, that thermal fluctuations in $\mathrm{Tw}$ and $\mathrm{Wr}$ are not coupled. Experimental measurements by Boles et al. (1990) determined essentially the same results for the parition of $\Delta \mathrm{Lk}$ into $\Delta \mathrm{Tw}$ and $\mathrm{Wr}$.

Knowing how $\mathrm{Tw}$ varies with $\mathrm{Lk}$ allows one to estimate the average twist angle between base pairs as a function of superhelix density, and thus to relate the torsion constant to an important molecular property of the DNA. Tw may be defined as the sum of the twist angles around the entire DNA chain

$$
\mathrm{Tw} \equiv \frac{1}{2 \pi} \sum_{i=1}^{\mathrm{N}} \phi_{i}
$$

where $\phi_{i}$ is the azimuthal twist angle in radians between base pairs $i$ and $i+1$, and $\mathrm{N}$ is the number of base pairs. The average twist angle is simply given by $\langle\phi\rangle=2 \pi \mathrm{Tw} / \mathrm{N}$. The equilibrium twist angle of relaxed DNA is $34.45^{\circ}$ ( 0.6013 radians), based on three very similar estimates of the helical repeat using different methodologies 
(Rhodes and Klug, 1980; Shore and Baldwin, 1983a; Wang, 1979). The equilibrium twist angle of a molecule with linking deficit $\Delta \mathrm{Lk}$ is therefore given by

$$
\langle\phi\rangle=\frac{\mathrm{E}}{\mathrm{N}} 2 \pi \Delta \mathrm{Lk}+0.6013
$$

where $F$ is the partition factor, equal to the fraction of $\Delta L k$ which ends up in Tw. The range of equilibrium twist angles in the DNA samples studied here, assuming $F=0.33$, ranges from $33.0^{\circ}$ to $34.9^{\circ}$, corresponding to superhelix densities of -0.123 to +0.042 . It is important to note that as $F$ decreases, so too does the range of equilibrium twist angles of our topoisomer samples.

The potential surface for base pair twisting is plotted in Fig 2.14 for $F=0.33$ at three different superhelix densities: $\sigma=0.00$, 0.084 , and +0.042 . Decreasing the magnitude of $F$ has two effects on the potential surface. First, the equilibrium twist angle changes more slowly as a function of superhelix density as $F$ decreases. Less of the change in Lk goes into Tw. Second, the anharmonicity of the potential surface is augmented as $F$ decreases. In effect, the slope of a plot of torsion constant vs. equilibrium twist angle is steeper when $F$ is small than for the case when $F=1.0$. This increase in the rate of change of the torsion constant as a function of twist angle is balanced, to some extent, by the fact that the equilibrium angle changes more slowly as $\mathrm{F}$ decreases.

Both the Barkley-Zimm and Allison-Schurr theories are based on a description of the DNA as a harmonic oscillator. Clearly, the data presented here demonstrate that this is not the case. The question arises, then, whether the non-ideal character of DNA 
Fig 2.14. Partition Factor $F=0.33$

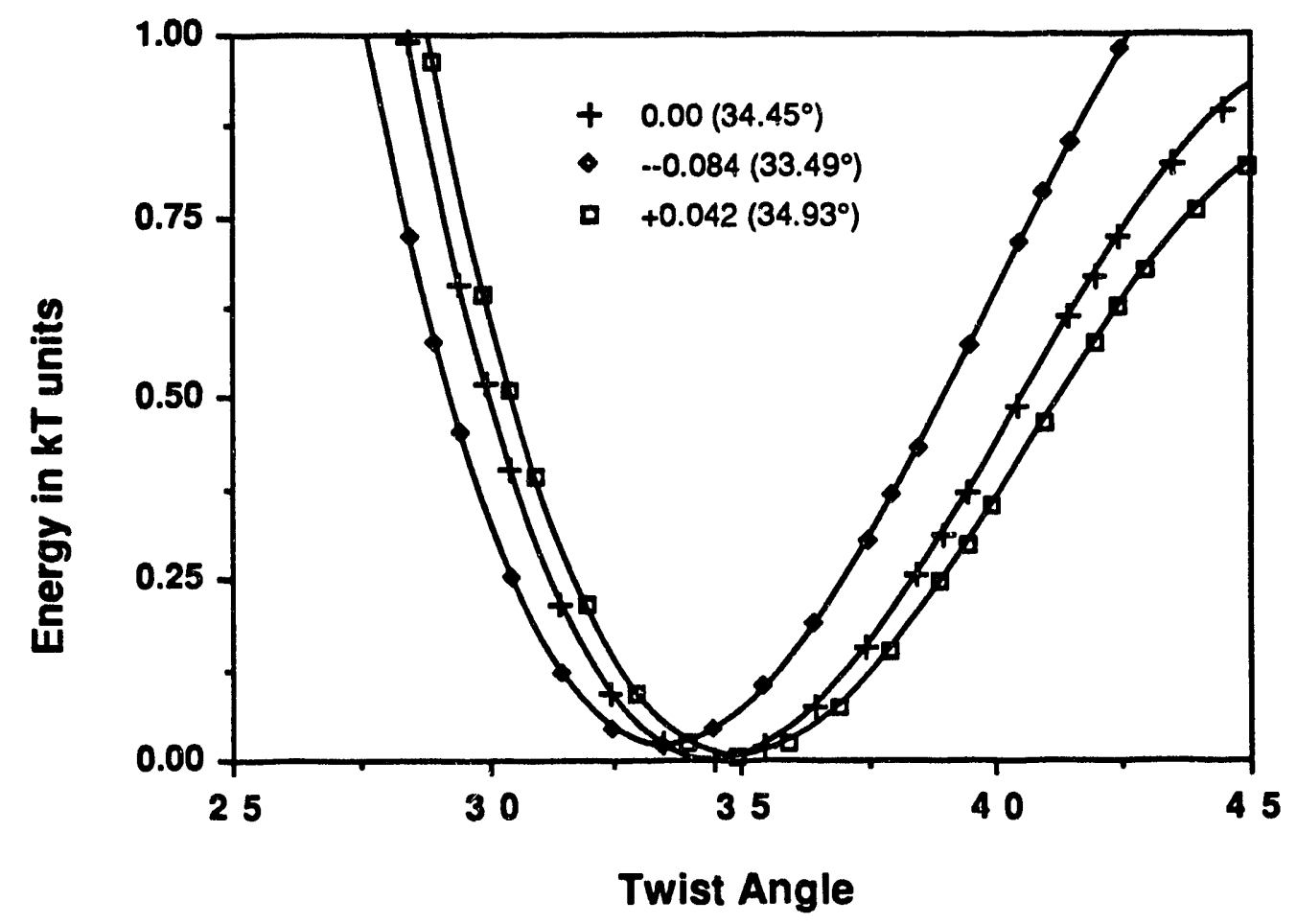

Fig 2.14. Plot of the potential energy surface for base pair twisting at superhelix densities of $0.00,-0.084$ and +0.042 for $F=0.33$. This plot is based on an extrapolation of the linear relationship of the torsion constant vs. superhelix density data in Fig 2.11 to large deviations in twist angle. Such an extrapolation has clear limits since, at extreme positive deviations in twist angle, the torsion constant is predicted to go to zero. The potential energy therefore also goes to zero. These plots clearly avoid this region. 
compromises our analysis of the FPA data based on these theories. The magnitude of the anharmonicity can be estimated by determining the magnitude of the cubic correction relative to the quadratic term in the potential energy expression when $\left(\alpha-\alpha_{0}\right)$ is \pm $5^{\circ}$, the rms deviation due to thermal energy. The potential energy function is that given in Fig 2.13A:

$$
\mathrm{U}=\frac{\mathrm{b}}{2}\left(\alpha-\alpha_{0}\right)^{2}+\frac{\mathrm{Fm}}{3}\left(\alpha-\alpha_{0}\right)^{3}
$$

From our data for relaxed DNA, $b=135$ and $m=-218$, both in $k T$ units per base pair. When $F=0.33$, the correction term represents a change of $\pm 15 \%$ in the height of the potential energy surface for the relaxed topoisomer at $U=1 / 2 k_{b} T$. An rms value is always greater than the mean, and so most twist angles deviate on average by less than $5^{\circ}$ from the equilibrium angle $\alpha_{0}$. As the twist deviation decreases, the cubic potential surface looks more like a simple harmonic surface. In fact, the measured torsion constant $k$ describes the shape of the potential surface in the limit of small displacements where the oscillation is harmonic. Although the nonlinear effects cannot be ignored, I conclude that our data analysis is not compromised by this cubic correction.

Future work. The experiments in this chapter raise a number of interesting questions relating to the structure of supercoiled DNA. I propose two projects, one computational and the other experimental, to explore the following questions: (1) How do changes in $\mathrm{Lk}$ partition into $\mathrm{Tw}$ and $\mathrm{Wr}$ ? and (2) How can an understanding of the torsional and bending stiffness of supercoiled DNA lead to an 
understanding of the tertiary structure of supercoiled molecules? I will briefly outline approaches to dealing with each of these problems.

Measuring changes in $T w$ as a function of superhelix density. To observe how $\Delta L$ k partitions into changes in $T w, I$ propose a fluorescence energy transfer experiment which will measure changes in the average base pair twist angle, $\langle\phi\rangle$, as a function of superhelix density. $\langle\phi\rangle$ can be related to $T w$ because $T w=(N / 2 \pi)$ $<\phi\rangle$. Conceptually, the experiment involves placing two different fluorophores a known distance apart (5 to 20 base pairs) on a supercoiled DNA. The fluorophores must be rigidly attached to the DNA and have suitable donor and acceptor properties for energy transfer measurements. The efficiency of energy transfer depends both on distance and on angular orientation between donor and acceptor in a well-understood manner. Changing the number of base pairs between donor and acceptor will alter both distance and orientation in a manner which depends on the rise per base pair and the local twist angle, respectively. Measuring the efficiency of energy transfer as a function of the number of base pairs between donor and acceptor should provide a measure of the twist angle, since the average rise per base pair is known from both crystallography and NMR. A similar set of measurements over a range of superhelix densities should yield the average twist angle as a function of $\mathrm{Lk}$.

The key aspect of this experiment is the ability to deliver donor and acceptor fluorophores to a precise location on a large, supercoiled DNA. To accomplish this task, I will synthesize two 
single-stranded, oligomeric DNA molecules with the ability to form triple-stranded helices at separate but closely space target sites on the supercoiled DNA. These oligomers will each contain a modified base which has a rigid orientation with respect to the helix, one to serve as a donor and the other as an acceptor. A single set of oligomers can serve for all of the experiments. The distance between donor and acceptor can be varied by inserting any number of base pairs between the oligomer target sites on the plasmid.

Modeling the structure of supercoiled DNA. The structure of supercoiled DNA is generally considered to be a plectonemic superhelix. The equilibrium supercoiled structure, apart from fluctuations due to thermal energy, is in principle entirely determined by the bending and twisting stiffness of the double helix. Like base pair twist, bending can be modeled at the level of adjacent base pairs as a torsional spring (Fig 2.15). The energy to induce a bend at a base pair therefore varies with the square of the angular displacement, just as for twisting:

$$
\mathrm{U}_{\text {bend }}=\frac{1}{2} \beta\left(\theta_{i}-\theta_{0}\right)^{2}
$$

where $\beta$ is the bending constant. (Note that this expression treats the bending as an isotropic distortion and does not account for local variations in the helical structure of DNA. For the current purpose of tertiary structure predictions for large molecules, I expect this approximation to be valid.) 
Fig 2.15. The spring model for DNA base pair bending

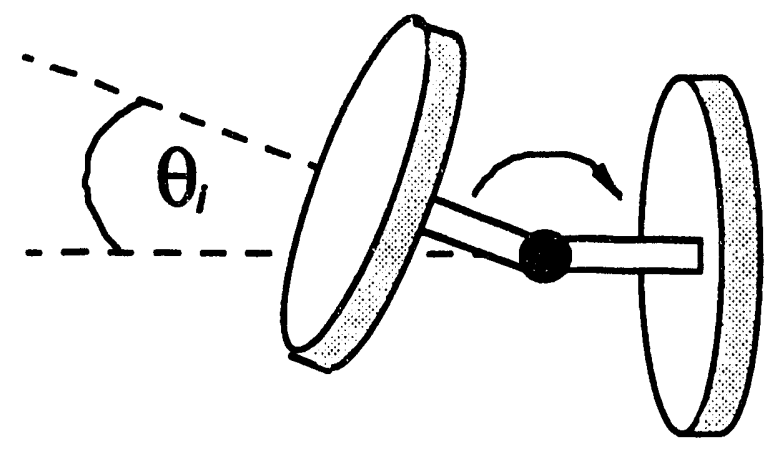

Fig 2.15. Adjacent base pairs are represented by disks separated by a torsion spring (filled circle). The equilibrium bending angle, in the absence of superhelical strain, is $\theta_{O}=2 \pi / \mathrm{N}$, which yields a circular molecule. The sum of the bending energy of individual base pairs determines the total bending energy and is related indirectly to Wr.

The minimum in bending energy, for a constant total amount of bending, will occur when all of the base pair bending angles $\theta_{i}$ are the same. Such a DNA molecule will describe a continuous space curve with curvature of constant magnitude. We are currently developing an analytical expression for continuous, closed curves of constant curvature which will serve as a model for plectonemically supercoiled DNA (J. Hearst, P. Selvin, and D. Cook). The Wr of any specific curve can be evaluated rigorously by a Gauss integral which, for a fixed value of $\mathrm{Lk}$, also determines $\mathrm{Tw}$. The total internal energy of such a model structure is then given by

$$
U_{\text {intermal }}=\frac{N}{2} \kappa\langle\phi\rangle^{2}+\frac{N}{2} \beta\left\langle\left.\theta\right|^{2}\right.
$$


where $\alpha$ and $\beta$ are the twisting and bending constants per base pair, respectively, and $\mathrm{N}$ is the total number of base pairs in the molecule. For a given value of $\mathrm{Lk}$, we will generate a family of curves consistent with the constraint of constant curvature. The internal energy of each structure can be calculated after determining Wr by applying the above formula. The equilibrium structure will be that which has the lowest internal energy. For this calculation, the experimentally measured variation of torsion constant with $\mathrm{Lk}$ will be used. We also have data which demonstrates that the bending constant $\beta$ does not vary with superhelix density (P. Selvin, M. Klein, and J. Hearst, unpublished data).

This simulation should be valuable for several reasons. First, computer generated images of the space curves may provide some physical insight into the tertiary structure of supercoiled DNA. Predictions from the model may therefore provide an impetus to further experiments. Second, results of the model can be directly compared to the experimental results for the variation of twist angle with Lk. Since the model is based on the simple mechanical properties of individual base pairs, comparison to experimental results may yield important insights into the local structure of supercoiled DNA. 


\section{Chapter 3}

\section{Chromosomal Supercoiling in Rhodobacter capsulatus during the Shift from Aerobic to Anaerobic Photosynthetic Growth}

In the photosynthetic bacterium Rhodobacter capsulatus, the enzyme DNA gyrase has been implicated in the expression of genes for anaerobic metabolic processes such as nitrogen fixation and photosynthesis. To assess the involvement of supercoiling in anaerobic gene expression, I have developed an assay to detect in vivo changes in superhelicity of small regions of the bacterial chromosome. The method is based on the preferential intercalation of psoralen into supercoiled versus relaxed DNA, and the sensitivity of the assay has been demonstrated in vivo on chromosomal fragments from 2 to 10 kilobases in size. In experiments with inhibitors of gyrase, the reactivity of individual chromosomal fragments to psoralen decreases by a factor of 1.8 compared with DNA from control cultures. I have used the assay to determine whether there is a change in superhelicity near the genes coding for essential proteins for photosynthesis upon a shift from respiratory to anaerobic photosynthetic growth. For comparison, I have also examined a restriction fragment containing the $f b c$ operon, which codes for the subunits of cytochrome $b c_{1}$, a membrane-bound electron transport complex utilized during both aerobic and anaerobic photosynthetic growth. During this shift, the puf and $p u h$ mRNAs, coding for structural polypeptides of the photosynthetic 
apparatus, underwent a six- to eight-fold induction, while the amount of mRNA from the $f b c$ locus remained constant. However, there is no stable change in the superhelicity of either the genes for photosynthesis or those for the $b c_{1}$ complex during this metabolic transition. Kinetic experiments, using a flow irradiation system and actively growing cells, indicate that the DNA downstream from the heavily transcribed $p u f$ and $p u h$ genes is transiently overwound upon induction of transcription, whereas the adjacent DNA in the transcribed region is not. The requirement for gyrase is interpreted, therefore, in terms of the twin domain model, in which the DNA downstream from heavily transcribed genes is overwound by the elongating polymerases, and gyrase functions to remove these positive supercoils.

\section{Introduction}

A recent model for the general control of gene expression for aerobic and anaerobic metabolism has postulated a role for DNA topology in regulating the transition between metabolic modes (Yamamoto and Droffner, 1985). The model proposes that DNA gyrase, which introduces negative supercoils into the chromosome, is essential for anaerobic gene expression, while topoisomerase I, which relaxes the chromosome, is required for expression of genes for aerobic metabolism. This hypothesis is based on the isolation in Salmonella typhimurium of obligate aerobes with mutations in one of the genes for DNA gyrase ( $g y r A$ or $g y r B$ ) and of obligate anaerobes whose mutations map to the gene for topoisomerase I (topA). This model of a supercoiling-induced switch in metabolic 
modes has been extended to other facultative anaerobes as well, througb the study of the effects of gyrase inhibitors on expression of essential genes for anaerobic metabolism. In Klebsiella pneumoniae, expression of the nifHDK genes, which code for nitrogenase and nitrogenase reductase, is blocked by drugs which inhibit gyrase (Kranz and Haselkorn, 1986), while in Bradyrhizobium japonicum, expression of enzymes for hydrogen metabolism, an anaerobic process, is also repressed by drugs which target gyrase (Novak and Maier, 1987).

This model of supercoiling-induced regulation of global patterns of gene expression has recently been extended to a variety of stress-related alterations in bacterial gene expression (Bhriain et al., 1989; Dorman et al., 1988; Dorman et al., 1990). These environmental factors which affect gene expression include $\mathrm{pH}$, osmolarity, temperature, and oxygen partial pressure. Studies have been performed primarily in $E$. coli and $S$. typhimurium utilizing the many genetic and biochemical tools developed for these organisms. As outlined in Chapter 1, the complexity of the results precludes a simple conclusion, in my opinion, but the model is an important current hypothesis in studies of bacterial physiology and gene regulation.

For the purple photosynthetic bacterium, Rhodobacter capsulatus, recent papers have documented the inhibition of anaerobic gene expression by drugs which inhibit gyrase. Under anaerobic conditions in the light, $R$. capsulatus develops an extensive photosynthetic membrane system in which pigment-protein complexes carry out light-driven electron transport to generate 
metabolic energy. $R$. capsulatus is also capable of nitrogen fixation under anaerobic, nitrogen-limiting conditions. Kranz and Haselkorn (1986) have shown that synthesis of the $R$. capsulatus nifHDK gene products is inhibited upon a five hour treatment with the gyrase inhibitor novobiocin, whereas synthesis of most major soluble proteins appears to be unaffected, as judged by $3 \mathrm{H}$-labeled amino acid incorporation and sodium dodecyl sulfate-polyacrylamide gel electrophoresis (SDS-PAGE).

In a study of mRNA accumulation from essential genes for photosynthesis, Zhu and Hearst (1988) showed that inhibition of gyrase results in the loss of detectable mRNA from the puhA locus, which codes for the $\mathrm{H}$-subunit of the photosynthetic reaction center, and from the puf operon, which codes for pigment-binding proteins in both the reaction center and light harvesting I antenna. Loss of detectable puhA mRNA occurred within 15 min after treatment with gyrase inhibitors, a time comparable with the half-life of the message. In contrast, mRNA levels for the $f b c$ operon coding for the cytochrome $b c_{l}$ complex, which is utilized for both respiration and photosynthesis, is unaffected by gyrase inhibitor treatment. The rapidity of the decrease in puhA and puf mRNA has been interpreted to imply that the observed effects result directly from gyrase inhibition and are not a secondary response to drug treatment (Zhu and Hearst, 1988).

An attractive hypothesis is that DNA superhelicity in the region of the genes for photosynthesis is altered by DNA gyrase, leading to repression or derepression of transcription (Zhu and Hearst, 1988). This model might explain why most of the known 
essential genes for photosynthesis are clustered on a $46 \mathrm{~kb}$ section of the chromosome (Marrs, 1981) as shown in Fig 3.1. Because the bacterial chromosome has been shown to be composed of topologically independent domains of torsionally strained DNA (Sinden et al., 1980; Worcel and Burgi, 1972), the photosynthesis cluster might conceivably be contained on a single domain and thus be subject to facile regulation by gyrase. Topological isolation of the photosynthesis gene cluster might allow it to be regulated by gyrase independently of other regions, such as the $f b c$ locus.

Heretofore, only an overall change in superhelicity of chromosomes has been amenable to analysis either by ethidium titration (Drlica and Snyder, 1978) or by isotopically labelled psoralen binding (Sinden et al., 1980). These methods allow one to detect changes in bulk chromosomal superhelicity but cannot test whether the torsional state of several domains might change independently without altering the overall superhelical density of the chromosome. An assay is described here using psoralen crosslinking to detect changes in the superhelicity of small segments of the chromosome.

The results presented here indicate that there is no stable change in the unrestrained superhelicity in the photosynthesis gene cluster during a shift in which genes for photosynthesis are induced five- to eight-fold. Similarly, there is no stable change in the superhelicity of DNA coding for the cytochrome bcl complex. The data thus do not support a model in which gyrase activates gene expression by adjusting local superhelix density under anaerobic conditions. Additional kinetic experiments suggest that 
Fig 3.1. A physical genetic map of the photosynthesis gene cluster from Rhodobacter capsulatus. BamHI restriction sites are denoted by solid circles, and fragment designations are shown in capitai letters. Bacteriochlorophyll biosynthesis genes $(b c h)$ are indicated by stippled regions, and carotenoid biosynthesis genes (crt) are indicated by crosshatched regions. Directions of transcription for the puf and $p u h$ genes are denoted by the arrows. Note that $35 \mathrm{~kb}$ of DNA separates the puf and $p u h$ promoter regions even though both operons code for structural proteins of the photosynthetic reaction center. 
$\varepsilon \varepsilon \mathrm{I}$

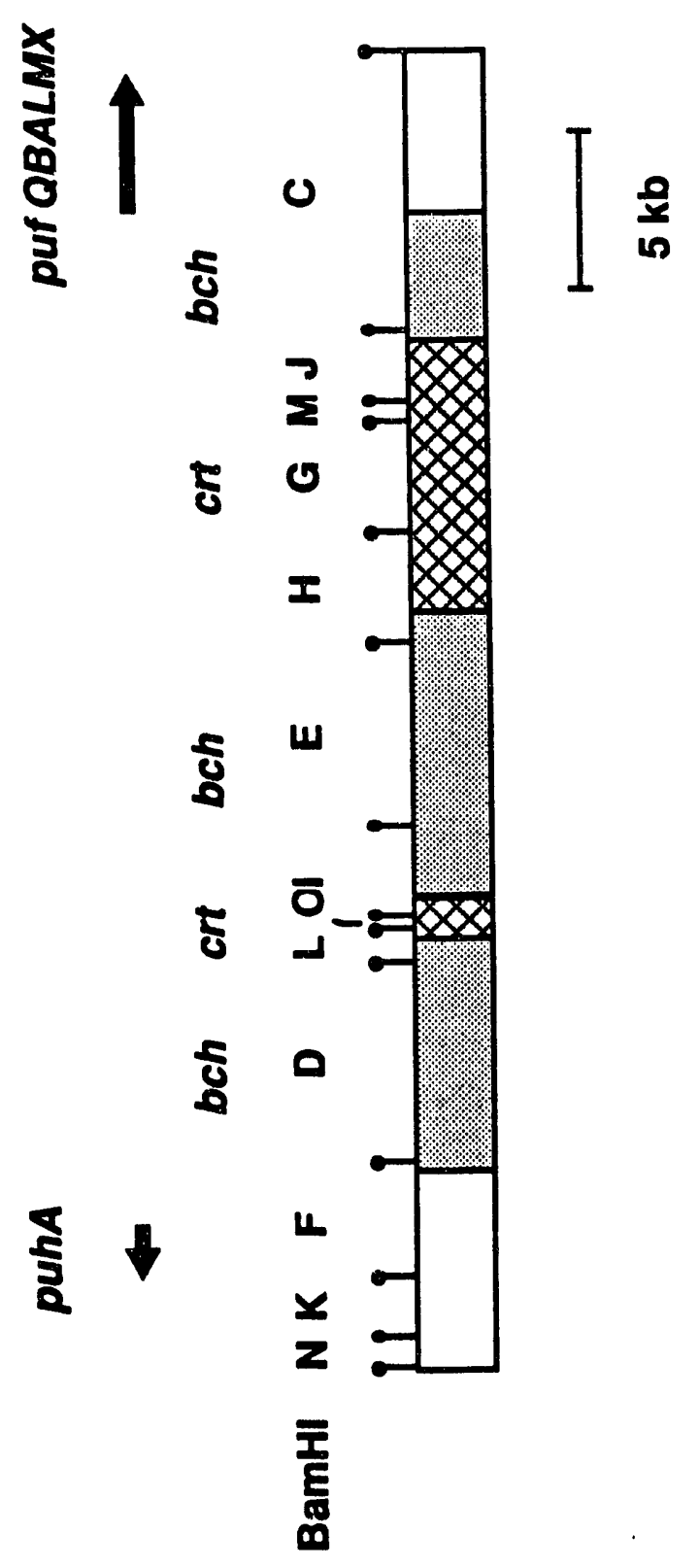


induction of photosynthetic metabolism results in the introduction of positive supercoils downstream from the highly expressed genes for the reaction center subunits of the photosynthetic apparatus. The requirement for DNA gyrase in the transcription of anaerobically induced genes may therefore result from the need for a swivel near these heavily transcribed regions of the chromosome.

\section{Materials and Methods}

Bacterial strains and growth. $R$. capsulatus strain SB1003 was grown in $150 \mathrm{ml}$ of RCV medium (Weaver et al., 1975) in $250 \mathrm{ml}$ side-arm flasks at $32^{\circ} \mathrm{C}$. Aerobically grown cultures were sparged in the dark with a mixture of $\mathrm{N}_{2}, \mathrm{O}_{2}$ and $\mathrm{CO}_{2}$ in a ratio of 80:20:2. Photosynthetic cultures were illuminated at approximately $15 \mathrm{~W} \cdot \mathrm{m}$ 2 by a bank of General Electric lumiline lamps and sparged with a mixture of $\mathrm{N}_{2}$ and $\mathrm{CO}_{2}$ in a ratio of 80:2. Gas flow rates were approximately $250 \mathrm{~cm}^{3} \cdot \mathrm{min}^{-1}$ for each culture. Gas flow rate and composition were controlled using a Matheson Gas Products Multiple Dyna-blender, model 8219. Growth rates were monitored using a Bausch \& Lomb Spectronic 21 at $680 \mathrm{~nm}$.

Irradiation of cells. Logarithmically growing cultures were quickly cooled in an EtOH-dry ice bath and pelleted for $6 \mathrm{~min}$ at $12000 \mathrm{xg}$ in an SS34 rotor. All subsequent steps were performed in dim light to eliminate undesired TMP crosslinking. Cells were resuspended in an ice-cold buffer containing $50 \mathrm{mM}$ Tris. $\mathrm{HCl}$ (pH 8), $10 \mathrm{mM}$ EDTA and $50 \mathrm{mM} \mathrm{NaCl}$ (TES) and $0.25 \mu \mathrm{g} \cdot \mathrm{ml}^{-1} \mathrm{TMP}$ for $10 \mathrm{~min}$ on ice. Irradiations were performed on $6 \mathrm{ml}$ samples in a 6-well tissue 
culture plate on ice. Long wavelength UV light at an intensity of 2 $\mathrm{mW} \cdot \mathrm{cm}^{-2}$ was provided by a bank of black light bulbs (The

Southern New England Ultraviolet Co., $\lambda_{\max }=350 \mathrm{~nm}$ ). Irradiated samples were pelleted and resuspended in a lysis solution consisting of $0.5 \%$ SDS, $10 \mathrm{mM}$ EDTA and $23 \mu \mathrm{g} \cdot \mathrm{ml}^{-1}$ proteinase $\mathrm{K}$ and stored at $-70^{\circ} \mathrm{C}$ for later use. For flow irradiations, cultures were maintained at $32^{\circ} \mathrm{C}$ and sparged with an appropriate mixture of gases. A continual stream of cells was removed from the culture at a rate of $2 \mathrm{ml} / \mathrm{min}$ and mixed with an equal flow of RCV medium containing TMP. After mixing for $2 \mathrm{~min}$ in flow, cells were streamed past the beam of a UV-enhanced, argon ion laser (Spectra Physics model 2045-15) and irradiated with a $5 \mathrm{~W}$ beam for approximately $5 \mathrm{sec}$. Irradiations were performed in a foil-wrapped flow cuvette to maximize utilization of the light. Samples were collected on ice and processed as described above.

DNA isolation. Cells were lysed by three freeze-thaw cycles between a $37^{\circ} \mathrm{C}$ bath and dry ice. After a $20 \mathrm{~min}$ incubation at $37^{\circ} \mathrm{C}$, DNA was extracted once with phenol:chloroform:isoamyl alcohol (25:24:1) and twice with chloroform:isoamyl alcohol (24:1). All steps though the first chloroform extraction were performed in dim light. Nucleic acids were precipitated with $\mathrm{EtOH}$ and resuspended in $0.5 \mathrm{ml}$ TES. RNase A digestion $\left(30 \mu \mathrm{g} \cdot \mathrm{ml}^{-1}\right)$ for 15 $\min$ at $37^{\circ} \mathrm{C}$ was followed by proteinase $\mathrm{K}$ digestion $\left(50 \mu \mathrm{g} \cdot \mathrm{ml}^{-1}\right)$ for $30 \mathrm{~min}$ at $55^{\circ} \mathrm{C}$. Samples were again extracted with phenol:chloroform:isoamyl alcohol followed by chloroform:isoamyl alcohol and EtOH precipitated. Before restriction, samples were 
irradiated with $36 \mathrm{~kJ} \cdot \mathrm{m}^{-2} \mathrm{UV}-\mathrm{B}$ to react any TMP monoadducts in potentially crosslinkable sites.

Gel electrophoresis and Southern blotting. Gels and DNA samples were handled essentially according to the method of Vos and Hannawalt (1987). DNA (4 to $5 \mu \mathrm{g}$ ) was denatured with 0.1 vol $1 \mathrm{M}$ $\mathrm{NaOH}$ at $55^{\circ} \mathrm{C}$ for $2 \mathrm{~min}$, rapidly neutralized with 0.12 vol $1 \mathrm{M}$ Tris $\cdot \mathrm{HCl}$ ( $\mathrm{pH} 4.2$ ), and placed on ice. Samples were run on Trisphosphate agarose gels and blotted to nitrocellulose overnight. After hybridization and autoradiography, radioactive bands were cut from the filter, dissolved in $1 \mathrm{ml} \mathrm{EtOAc}$, and counted Opti-fluor (Packard Co) in a Packard model 3385 scintillation counter.

\section{Results}

An Assay for local changes in chromosomal superhelicity. DNA intercalators unwind the helix and therefore bind more readily to negatively supercoiled than to relaxed DNA (Bauer and Vinograd, 1968; Bauer and Vinograd, 1970). The psoralens are a class of intercalators which are capable of reacting photochemically with DNA (Cimino et al, 1985). Upon irradiation with long wavelength UV light (UV-A) psoralens form cyclobutane monoadducts and interstrand crosslinks with pyrimidine bases of the DNA. The rate of formation of these adducts has been shown to be a function of unrestrained DNA superhelicity both in vitro and in vivo (Hyde and Hearst, 1978; Sinden et al., 1980). Psoralen intercalation is not sensitive to supercoils restrained by wrapping of DNA with proteins. In experiments with Escherichia coli, Sinden et al. (1980) showed 
that the rate of addition to chromosomal DNA in control cells is nearly twice that of cells treated with the gyrase inhibitor novobiocin or irradiated with a $\gamma$-emitting ${ }^{60} \mathrm{Co}$ source. They utilized a radiolabeled derivative, $[3 \mathrm{H}]-4,5^{\prime}, 8$-trimethylpsoralen (TMP), to determine incorporation of the drug into bulk DNA. These experiments demonstrated convincingly that the bacterial chromosome contains unrestrained supercoils in vivo which result in torsional tension in the DNA helix.

The usefulness of psoralen for detecting changes in supercoiling in vivo can be extended by exploiting the ability of psoralen-crosslinked DNA to rapidly reanneal after a denaturation (Johnston et al., 1977). The method here measures the rate of TMP crosslinking to any specific DNA restriction fragment in the genome and is thus an assay for changes in local torsional tension (Fig 3.2). After in vivo crosslinking at low levels (less than one TMP crosslink per kilobase), genomic DNA is purified, restricted, and denatured. Under alkaline conditions ( $\mathrm{pH} 13$ ), crosslinked strands melt but are held in register by TMP so that they rapidly reanneal upon a return to neutral $\mathrm{pH}$. The crosslink provides a nucleation site for helix formation. Unmodified or monoadducted DNA, however, is irreversibly denatured in this procedure. Electrophoresis under native conditions separates crosslinked from uncrosslinked DNA, and the amount of crosslinking of any particular restriction fragment in a genomic digest can be quantified by Southern blotting (Vos and Hannawalt, 1987).

Fig 3.3 shows the effect of novobiocin-induced relaxation of specific restriction fragments in the TMP assay. $R$. capsulatus strain 
Fig 3.2. Schematic illustration of the TMP assay. (A) DNA is irradiated in vivo to a level of less that 1 crosslinkable TMP adduct per kilobase. After isolation, samples are reirradiated in vitro to ensure the maximum yield of psoralen crosslinks. (B) Following endonuclease restriction, DNA is denatured briefly with alkali. Upon neutralization, TMP-crosslinked DNA reanneals rapidly, whereas uncrosslinked DNA is irreversibly denatured. (D) Samples are electrophoresed in an agarose gel, blotted to nitrocellulose, and probed for the specific sequence of interest. The level of crosslinking for any fragment in the genome can be determined in this manner. XL: crosslinked DNA; SS: single stranded DNA. 
Fig 3.2. The TMP Assay for Localized Changes in Supercoiling.
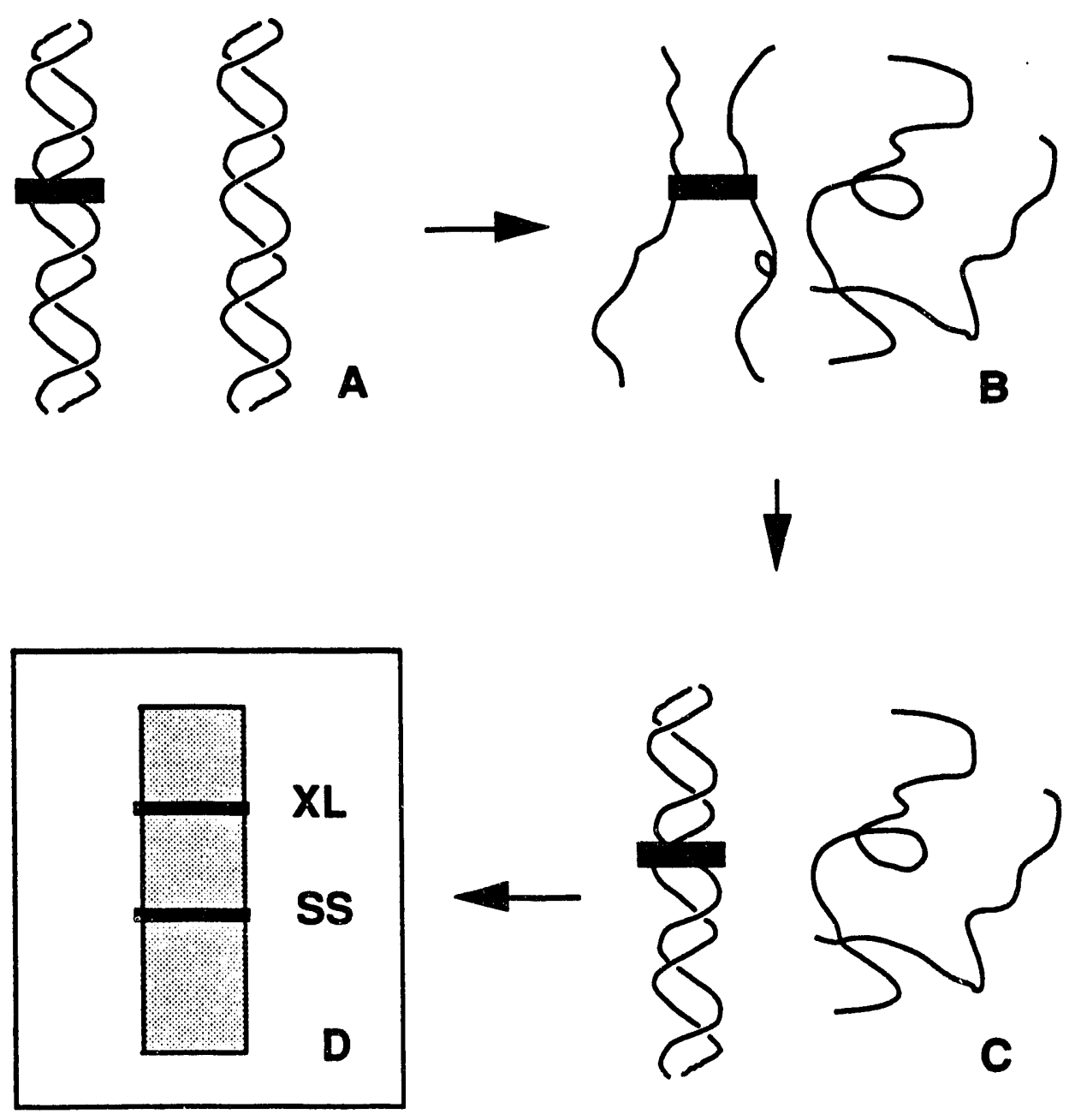
Fig 3.3. Southern blot of genomic DNA irradiatedin vivo for various times, restricted and probed with the BamHI-K fragment of the photosynthesis cluster. Before irradiation cells were grown without ( (-) NOVO) or with ( (+) NOVO) for $15 \mathrm{~min}$. Irradiation times, in minutes, are given above each lane. Note that for comparable times, novobiocin treatement reduces the yield of crosslinked DNA. 
Fig 3.3. Southern Blot of an in vivo irradiation time course

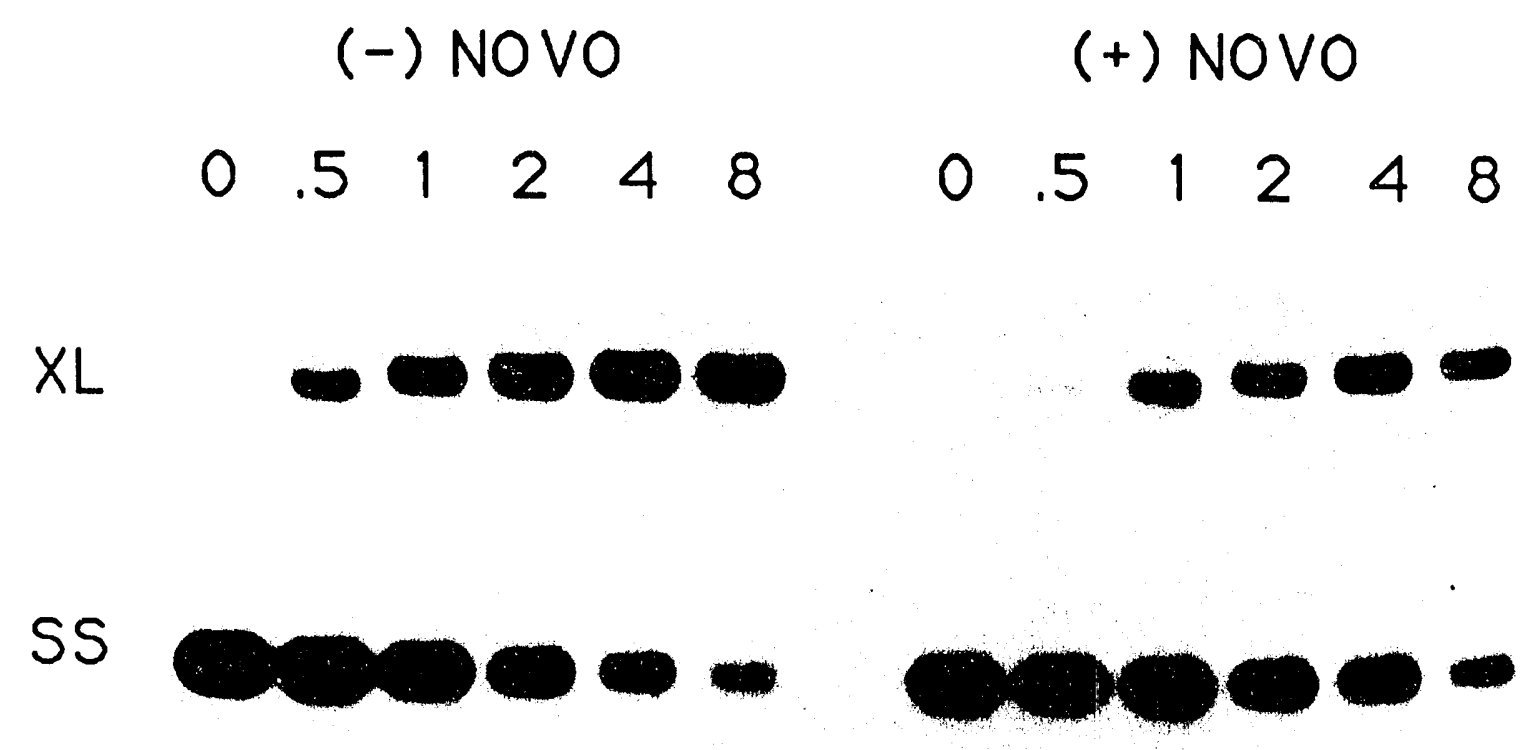


SB1003 was grown aerobically in the dark as described in Materials and Methods. A portion of the culture was quickly cooled, pelleted and resuspended in an ice cold buffer containing TMP. Cells were incubated in the dark on ice to allow the TMP to equilibrate with intracellular DNA and were subsequently irradiated while on ice. The remainder of the culture was treated with novobiocin, harvested, and irradiated as described above. After isolation of genomic DNA and removal of free TMP by organic extraction, samples were reirradiated with UV-A to crosslink any psoralen monoadducts in potentially crosslinkable sites. Since the assay is specific for crosslinks, this in vitro irradiation step substantially increases the sensitivity and reproducibility of the assay. The DNA samples were then restricted and denatured immediately prior to gel electrophoresis. Neutralized samples were run on native agarose gels, blotted, and the fragments of interest were visualized by hybridization.

These blots characteristically contain two bands in each lane: a lower band which runs at the position of the single stranded DNA and an upper crosslinked band which migrates at the position of double stranded DNA. Samples incubated with TMP but not irradiated show no detectable crosslinked band (Fig 3.3, lanes 1 and 7). Longer exposure to light results in a higher percentage of crosslinked DNA. Most importantly, the rate of appearance of crosslinked DNA is notably slower when cells are treated with novobiocin, indicating that less TMP is intercalated in the relaxed chromosome. 
Blots can be quantified by determining the fraction of counts at the single stranded position for each time point. When plotted on a semilog scale these data yield a linear relationship (Fig 3.4). This behavior would be expected for a Poisson-type process in which a single psoralen crosslink in a DNA fragment is sufficient to cause the DNA to run as a double strand. The ratio of crosslinking rates between control and novobiocin-relaxed DNA for restriction fragments in this experiment is about 1.8 (Fig 3.4). This ratio is identical to that observed by Sinden et al. (1980) upon treatment of $E$. coli with novobiocin or exposure to $\gamma$-irradiation, indicating a complete relaxation of free torsional tension in the chromosome. We have examined a number of BamHI restriction fragments from the photosynthesis gene cluster, including the BamHI.C, $-\mathrm{D},-\mathrm{F},-\mathrm{G},-\mathrm{H},-\mathrm{J}$, and $-\mathrm{K}$ (Fig 3.1). These restriction fragments contain genes coding for carotenoid and bacteriochlorophyll biosynthetic enzymes and for the structural polypeptides which bind these pigments and carry out the light reactions in the photosynthetic membrane (Taylor et al., 1983; Zsebo and Hearst, 1984). These fragments range in size between 2 and $10 \mathrm{~kb}$, and each is sensitive to the crosslinking assay in the presence of novobiocin. Larger fragments crosslink at a faster rate since they represent a larger target for crosslinking (Fig 3.4).

Time-course of novobiocin action. Certain species of gram negative bacteria, including $\dot{E}$. coli and $S$. typhimurium, are resistant to high doses $(>50 \mathrm{mg} / \mathrm{ml})$ of novobiocin. This resistance to the action of the drug is due to the exclusion of novobiocin by the outer membrane of enteric bacteria (Nikaido and Vaara, 1985). Like many small 
Fig 3.4 Irradiation of Cells with and without Novo

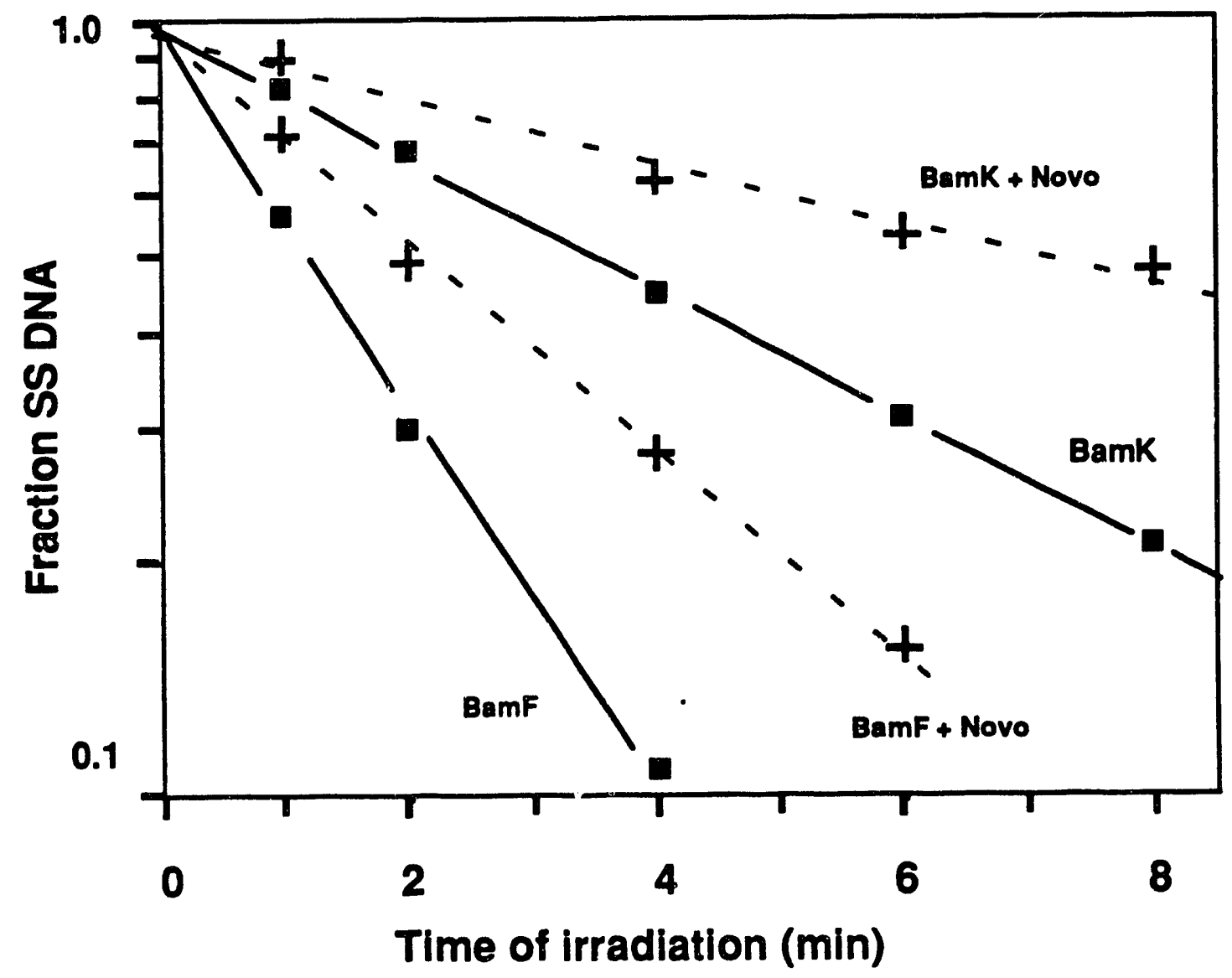

Fig 3.4. Quantitation of results from the Southern Blot in Fig 3.3 and a similar blot for the BamHI-F fragment from the photosynthesis gene cluster. Symbols: culture before the addition of novobiocin; + , novobiocin-treated culture. The ratio of rates from this and other graphs indicates a 1.8 -fold enhancement of TMP reactivity in the control culture. The BamHI-F fragment is twice the size of the BamHI-K and also twice as likely to react with TMP in this experiment. 
hydrophobic molecules, novobiocin is shielded from passing through the lipid bilayer of the outer membrane by the dense polysacharide-phosphate polymer attached to outer membrane lipids. This outer barrier is further stabilized by interaction with divalent cations such as $\mathrm{Mg}^{2+}$ and $\mathrm{Ca}^{2+}$ which are coordinated to the terminal phosphate groups of the lipopolysaccharide layer. This ability to exclude small hydrophobic molecules is thought to have evolved in enteric bacteria as a defence against the detergent action of bile salts in the gut of host organisms (Nikaido and Vaara, 1985). In $R$. capsulatus, a free living gram-negative bacterium, the lipopolysaccharide shield is absent. Both novobiocin and TMP appear to enter the cells with ease (Fig 3.3).

A typical time course of chromosome relaxation is shown in Fig 3.5. Cells were grown under steady state photosynthetic or aerobic conditions and novobiocin was added at time 0 . Aliquots were withdrawn at various intervals, quick cooled to $4^{\circ} \mathrm{C}$, and pelleted in a microfuge. The TMP equilibration and irradiation were performed as described previously. Data are shown for two adjacent restriction fragments of the photosynthesis gene cluster, the $4 \mathrm{~kb}$ BamHI-F fragment and the $2 \mathrm{~kb}$ BamHI-K fragment (Fig 3.1). The effect of the drug is readily evident after only five minutes, the earliest time measured, indicating that novobiocin quickly enters $R$. capsulatus. The maximum relaxation is at $10 \mathrm{~min}$ for the BamHI-K and $20 \mathrm{~min}$ for the BamHI-F, in both photosynthetic and aerobic cells. For most other fragments tested, the effect is maximal at $20 \mathrm{~min}$ (data not shown). This time course is similar to that observed by Drlica and Snyder (1978) in E. coli. 
Fig 3.5. Time-course of novobiocin action in aerobic and photosynthetic cells. Novobiocin was added at time 0 to cultures to a final concentration of $80 \mathrm{mg} / \mathrm{ml}$. Samples were quickly cooled, pelleted, and resuspended in TES buffer containing $0.25 \mathrm{mg} / \mathrm{ml}$ TMP. After a 10 min incubation on ice, cultures were irradiated with $2 \mathrm{~mW} / \mathrm{cm}^{2} \mathrm{UV}$-A for $4 \mathrm{~min}$. DNA was isolated and analyzed according to procedures in Material and Methods. A decrease in the Fraction Double Stranded indicates relaxation of negative supercoils in the chromosome. 
Fig 3.5A Novobiocin relaxation time course for $\mathrm{O} 2$ cells

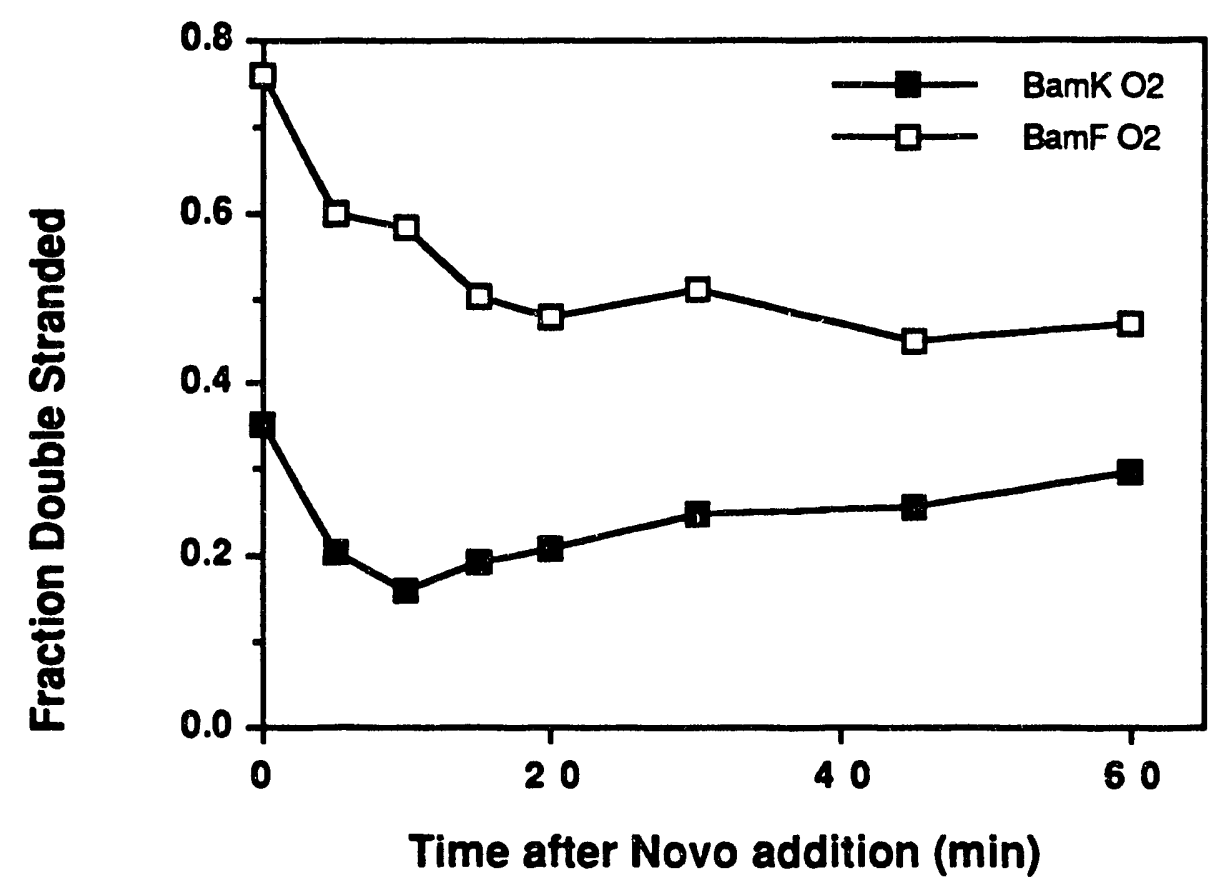

Fig 3.5B Novobiocin relaxation time course for PS cells

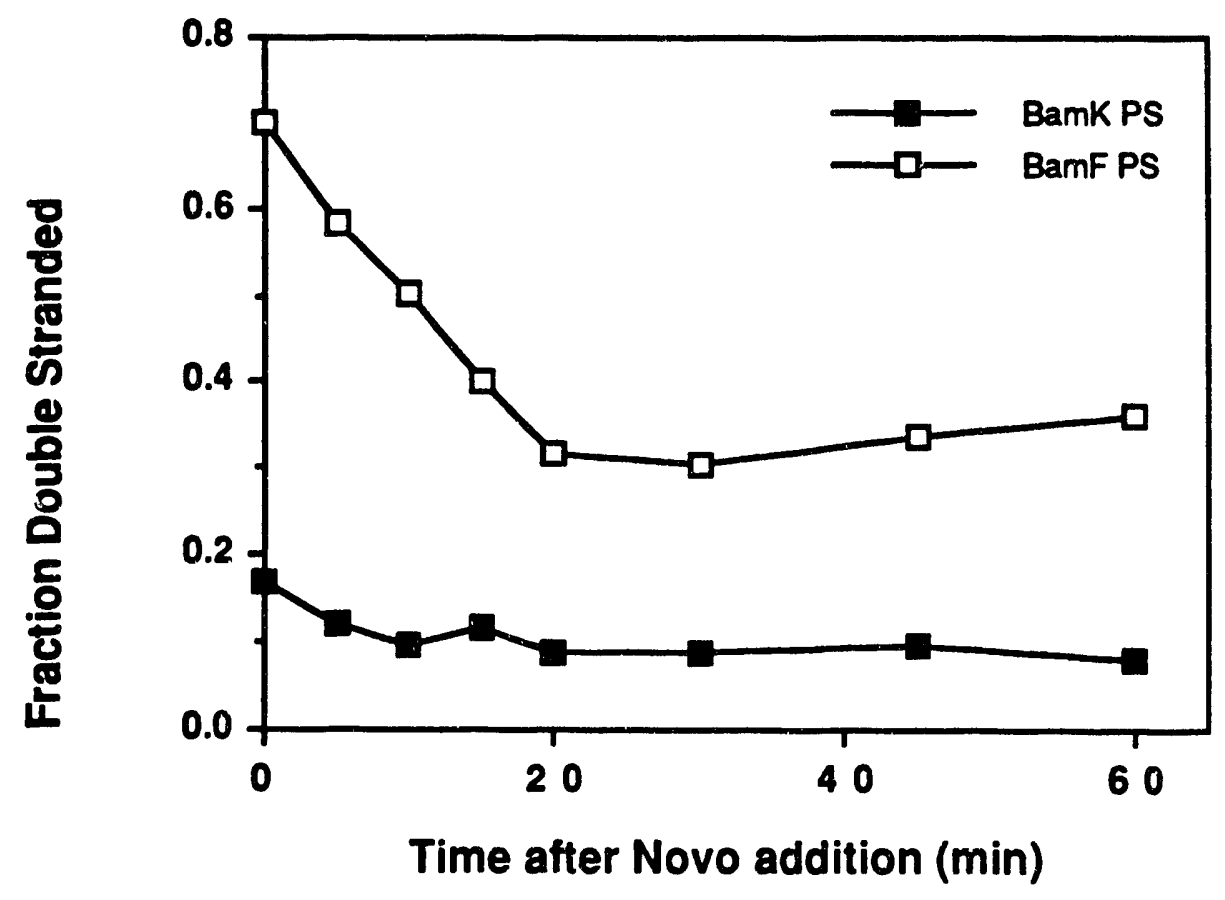


coli chromosome by the DNA gyrase inhibitor coumermycin.

Coumermycin has a mode of action similar to that of novobiocin (Gellert et al., 1976).

At long times, we observe some increase in psoralen reactivity, possibly indicating a recovery in torsional tension in the chromosome (Fig 3.5). This phenomena is also seen when other restriction fragments are probed, but the exact significance of this observation is not clear. Either metabolism of the drug or induction of additional gyrase activity may explain this result. Menzel and Gellert (1983) have shown in E. coli that treatment with gyrase inhibitors does increase the transcription of the genes for the enzyme and that this effect is mediated through changes in DNA supercoiling. Franco and Drlica (1989) have further demonstrated that low levels of gyrase inhibitors lead to an increase in negative supercoiling. The increase in supercoiling correlates with increased expression of the gyrA subunit of DNA gyrase. Thus, it is reasonable that $R$. capsulatus may have increased levels of DNA gyrase some time after treatment with gyrase inhibitors, and that this increase in gyrase could lead to a recovery in chromosomal supercoiling.

mRNA accumulation during the shift from aerobic to anaerobic photosynthetic growth. TMP is a hydrophobic and sparingly soluble compound (aqueous solubility of $0.6 \mu \mathrm{g} \cdot \mathrm{ml}^{-1}$ ) which, when added to an anaerobic cell culture, would preferentially reside in the extensive photosynthetic membrane system of $R$. capsulatus. This fact complicates any effort to directly compare aerobic and 
anaerobic cultures using the psoralen assay. Experiments were instead performed during a shift from aerobic to anaerobic conditions to alleviate any potential problems caused by sequestering of TMP in the membrane of photosynthetic cells. Cultures shifted from 20 to 0 percent oxygen exhibit little or no growth for the first hour and thereafter resume growth at a slightly higher rate than under aerobic conditions (Fig 3.6A). This result is in agreement with the work of Gray (1967), who showed that, in an identical shift from aerobic-dark to anaerobic-light conditions, Rhodobacter sphaeroides undergoes a $90 \mathrm{~min}$ lag phase. During the induction period, there are no significant increases in cell number or cell mass or in total cellular RNA, DNA, or protein content (Gray, 1967).

In the first two hours after the shift, transcription of the structural proteins for photosynthetic reaction centers (coded for by pufLM and $p u h A$ ) and the light harvesting I antennae (coded for by $p u f A B$ ) is induced approximately six- to nine-fold (Fig 3.6B and 3.6C). A time course of mRNA accumulation demonstrates that these genes are induced approximately $30 \mathrm{~min}$ after a rigorous aerobic to anaerobic shift and that mRNA accumulates to a maximum 90 min after the shift, approximately the same time at which cell growth resumes (Fig 3.6A). In comparison, mRNA for the cytochrome bc1 complex (coded for by the $f b c$ operon) does not change significantly from pre-shift levels (Fig 3.6B and 3.6C). This complex is utilized for respiratory as well as photosynthetic growth (Daldal et al., 1987) and is therefore already present in the cellular membrane. 
Fig 3.6. Response of Rhodobacter capsulatus to shift in growth conditions. (A) Growth curves for two different cultures upon a shift from aerobic to anaerobic photosynthetic growth conditions.

(B) mRNA accumulation for a shifted cell culture for $p u f L M, p u f B A$, $p u h A$, and $f b c F B C$ gene specific probes. Data shown were normalized to rFNA but similar results were obtained when normalized to total RNA. 
Fig 3.6

A. Growth of Cells after Photosynthetic Shift

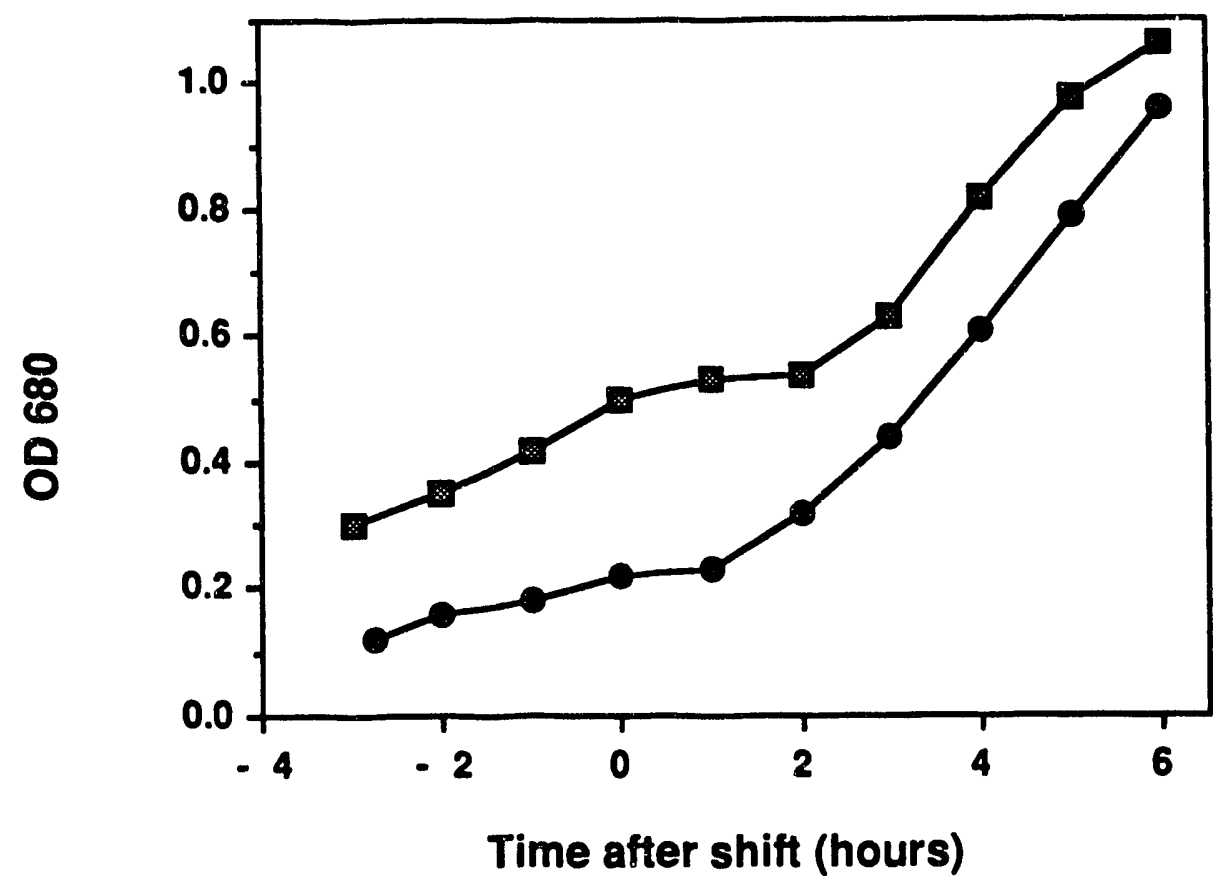

Fig 3.6 B. mRNA Accumulation After Shift

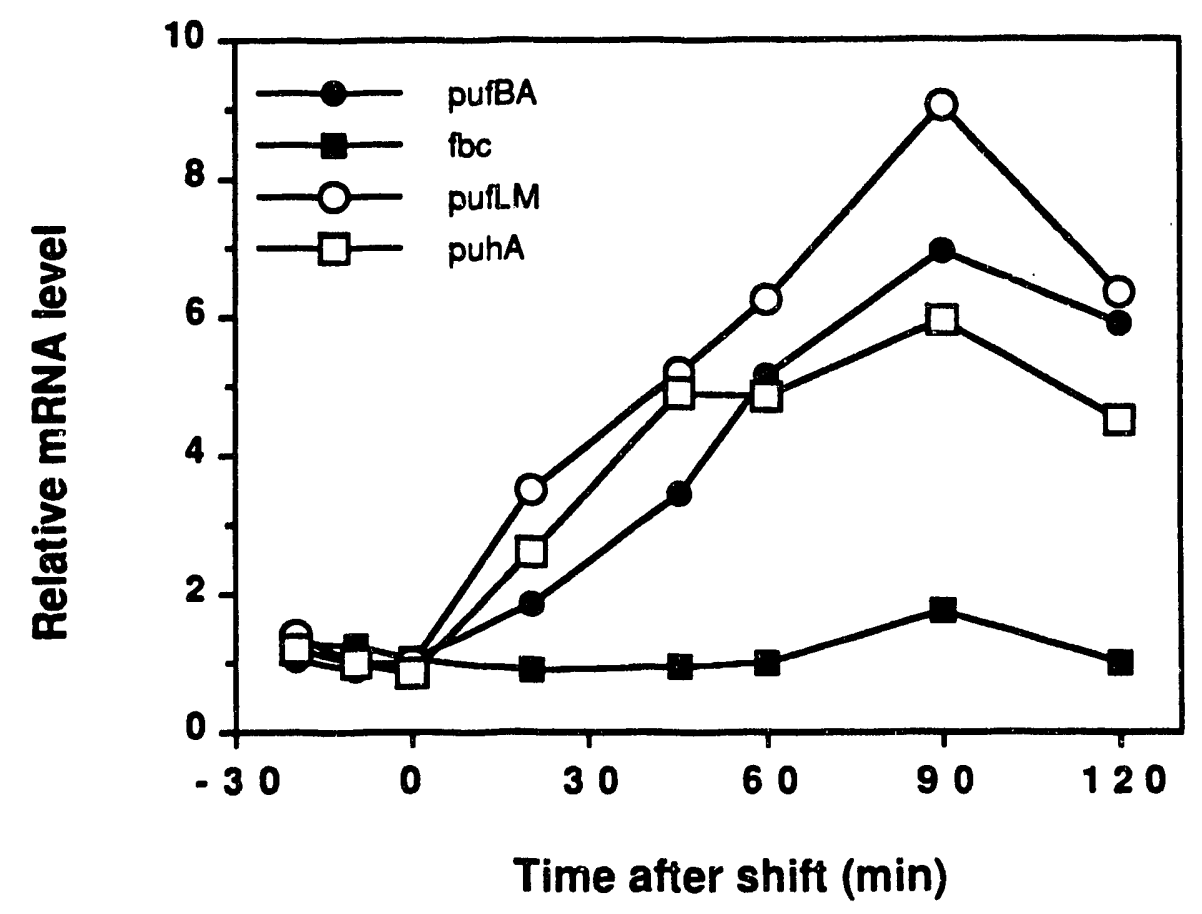


Chromosomal supercoiling during the induction of genes for photosynthetic metabolism. I have utilized our assay to test whether stable changes in superhelicity regulate transcription of genes for photosynthesis in the switch from aerobic to anaerobic metabolism. $R$. capsulatus was grown aerobically in the dark to mid-log phase. A portion of the culture was harvested and irradiated with UV-B while the remainder was shifted to anaerobic conditions in the light. Forty-five minutes into the shift, when the rate of mRNA accumulation for photosynthesis genes is maximal, the procedure was repeated on the induced cells. There is no detectable change in superhelicity for the DNA of the photosynthesis gene cluster (Fig 3.7). The BamHI-F fragment shown in Fig 3.7 contains the puhA gene which codes for the H-subunit of the photochemical reaction center, while the BamHI-K, a $2 \mathrm{~kb}$ fragment, is immediately downstream from puhA. I have also probed the BamHI-J, -G, and -D to confirm this result for other regions of the photosynthesis gene cluster (data not shown). Similarly, there is no detectable change in superhelicity of a $9 \mathrm{~kb}$ restriction fragment containing the genes for the $b_{1} 1$ complex (Fig 3.7). In $R$. capsulatus, the puf and puh genes are not genetically linked to the $f b c$ genes (Daldal, et al., 1987). Recent physical mapping of the $R$. capsulatus chromosome shows that $f b c$ is separated by more than $1 \mathrm{Mb}$ from the photosynthesis cluster (J. Williams and B. Marrs, Abstract from Molecular Biology of Membrane Bound Complexes in Phototrophic Bacteria, Freiburg, West Germany, August 1989). I have repeated this experiment three times and examined six different BamHI restriction fragments from the $R$. capsulatus chromosome. The deviation in crosslinking 
Fig 3.7 Crosslinking rates of 0 and PŞ-shifted culture

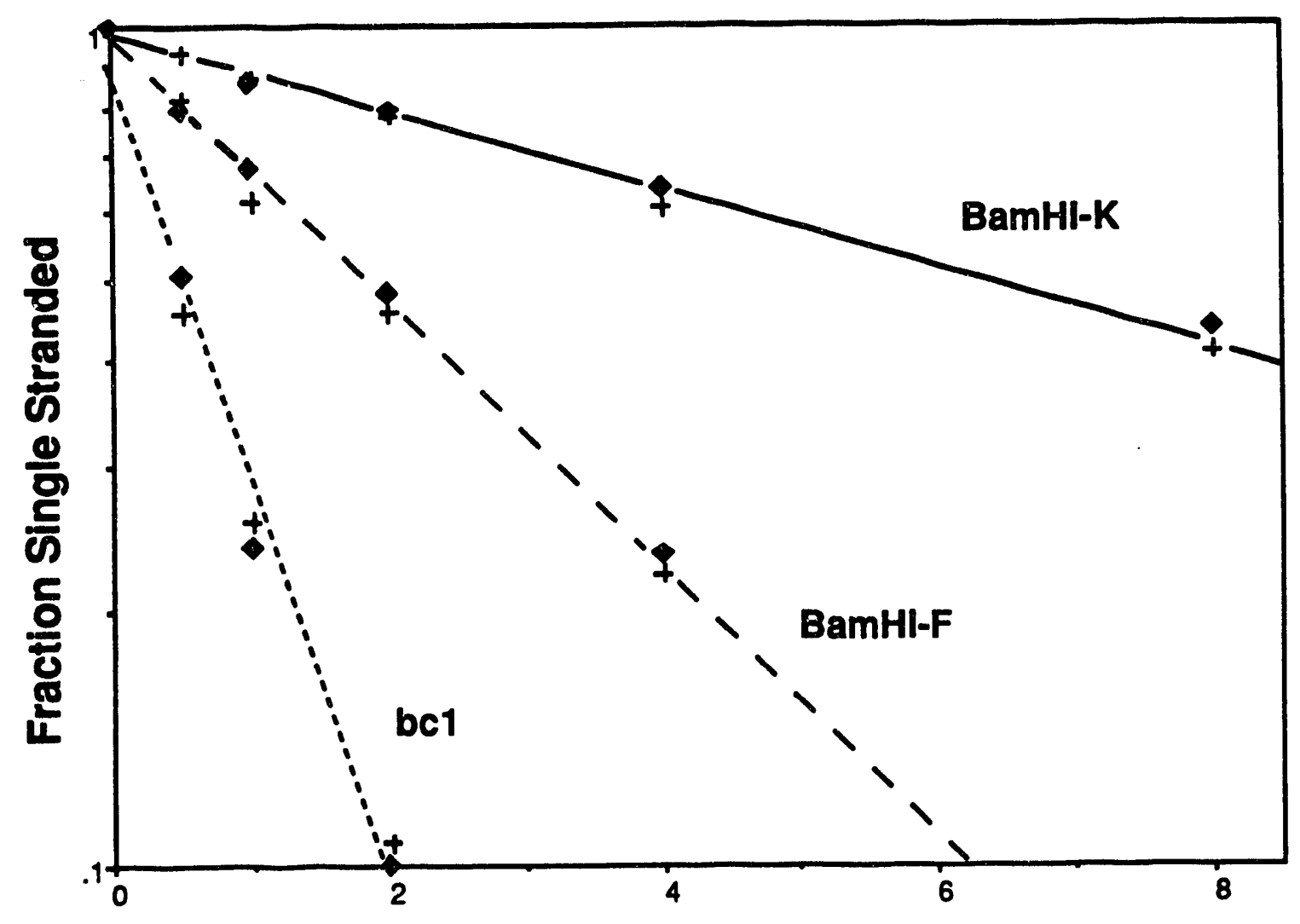

Time of Irradiation (min)

Fig. 3.7. Comparision of crosslinking rates of aerobic ( ) and anaerobic $(+)$ cells. The irradiation of shifted cells was performed 45 min into the adaptation period to photosynthetic growth. The BamHI $\mathrm{K}$ and $\mathrm{F}$ fragments are part of the photosynthetic gene cluster and are 2 and $4 \mathrm{~kb}$ in size, respectively. The $b c_{1}$ probe is a 9 kb restriction fragment contaioning all three genes from the $f b c$ operon. 
rates between aerobic and anaerobic cultures averaged $5 \pm 3$ percent. These results, therefore, demonstrate that neither highly regulated genes such as $p u f L M$ and $p u h A$ nor a constitutive gene such as $f b c$ undergoes a local change in superhelicity after a shift from aerobic to anaerobic photosynthetic growth.

Transient changes in chromosomal supercoiling during the induction of genes for photosynthesis. The experiments described thus far have been designed to ditermine whether stable changes occur in chromosomal DNA supercoiling during the induction of anaerobic metabolism. Cells were harvested in a particular metabolic state, resuspended in a cold buffer containing TMP, and irradiated. If there are stable changes in supercoiling, or changes in the supercoiling of particular chromosomal domains, I would expect to detect these changes based on the sensitivity of the assay to chromosomal relaxation by novobiocin. Transient changes in DNA supercoiling, such as those due to driven rotation of the DNA by RNA polymerase, might escape detection because of the cooling of cells during the TMP equilibration and irradiation periods.

In order to address the possibility of transient changes in DNA supercoiling on the chromosome, the experimental protocol was modified in several ways. To look at transcribing cells, the actively metabolizing bacterial culture was pumped past a light source, and irradiation times were drastically reduced from a maximum of 8 min under the previous protocol. The requirement for short irradiation times of cultures in flow necessitates the use of a high power, argon ion laser as shown in the schematic of the apparatus 
in Fig 3.8. Using the all-lines UV emission (330-363 $\mathrm{nm}$ ) of approximately $5 \mathrm{~W}$, irradiations are carried out in a foil-wrapped flow cuvette and are complete in approximately $5 \mathrm{sec}$. A second change in the experimental protocol is that the bacterial culture is no longer pre-equilibrated with TMP before irradiation. Instead, the bacterial culture is pumped into a small mixing chamber simultaneously with growth media containing TMP (Fig 3.8). This change eliminates the possible artifacts of growing cells in a TMPsaturated medium, because psoralen can be metabolized by bacterial cells (data not shown). The mixing of cells and TMP is kept constant at $2 \mathrm{~min}$, a time that was found to give maximal crosslinking of DNA. By keeping the time of mixing constant and collecting continuous fractions in flow, the crosslinking yield can be monitored during a shift of the culture from aerobic to anaerobic photosynthetis growth conditions.

The results obtained by this procedure differ strikingly from those detailed above. Restriction fragments immediately downstream from the puf and puh operons show a marked decrease in TMP reactivity following a shift from aerobic to anaerobic photosynthetic conditions (Fig 3.9). The TMP reactivity reaches a minimum 20 to $30 \mathrm{~min}$ after the shift and increases slightly thereafter. This decrease in psoralen reactivity is consistent with the idea that, downstream from heavily transcribed genes, the DNA is being relaxed or perhaps overwound as a result of driven rotation by RNA polymerase (see Fig 1.3). According to the twin domain model, one of the roles of gyrase is to restore the negative supercoils removed by transcription. If the ability of gyrase to 


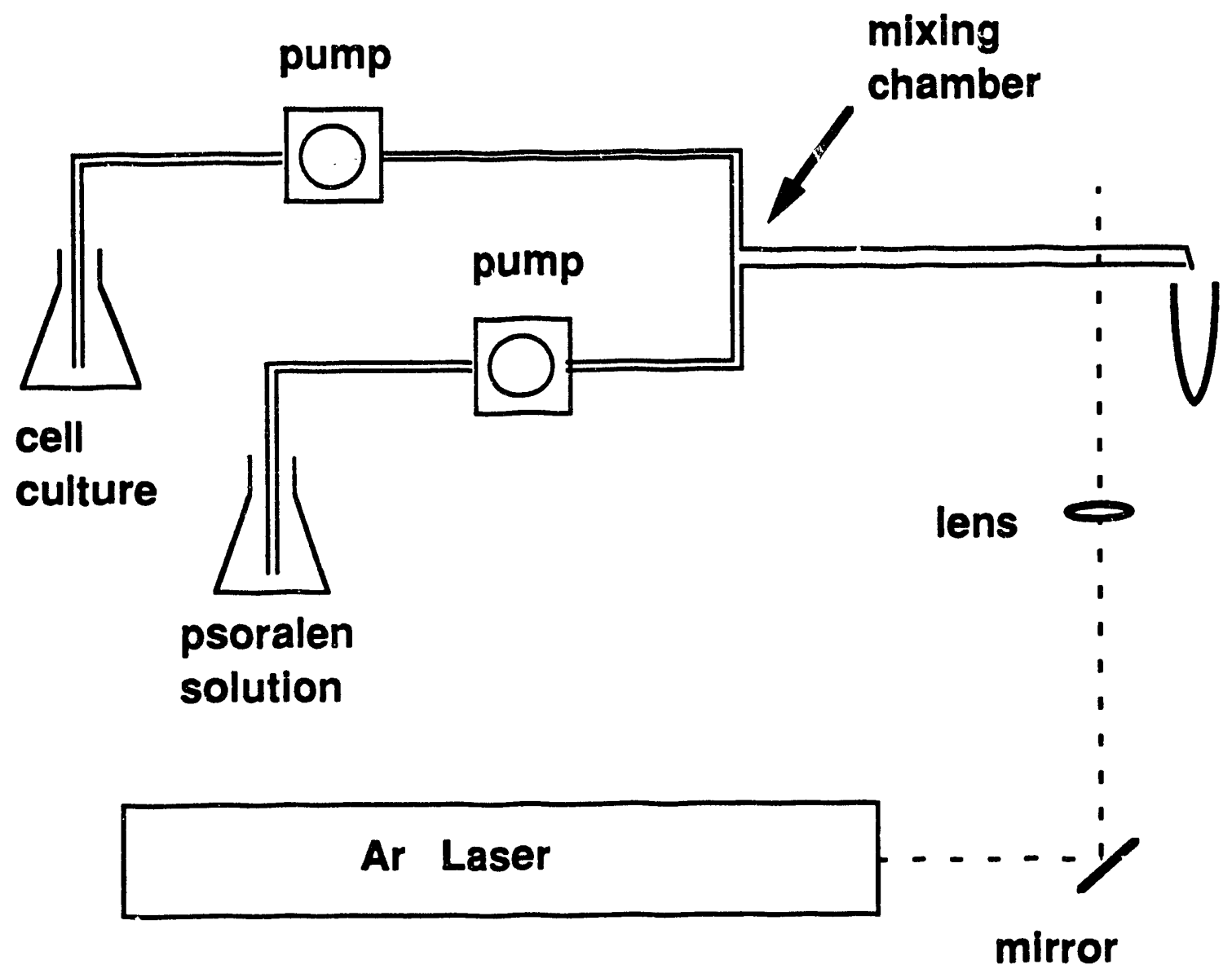

Fig 3.8. Flow irradiation apparatus. Cells are grown under appropriate gas mixtures and are withdrawn from the culture through a wide bore needle and a septum. Growth medium containing TMP is pumped at an identical rate into a mixing chamber. Cells mix with TMP for a total of $2 \mathrm{~min}$ until they reach the flow cuvette. In the cuvette they are irradiated with the $5 \mathrm{~W}$ all lines UV emission (330-363 $\mathrm{nm}$ ) from an argon ion laser. The total irradiation time is approximately $5 \mathrm{sec}$. Samples are collected on ice, spun down, resuspended in lysis buffer and immediately frozen for later work up. 
Fig 3.9 TMP Reactivity Downstream of Reaction Center Genes

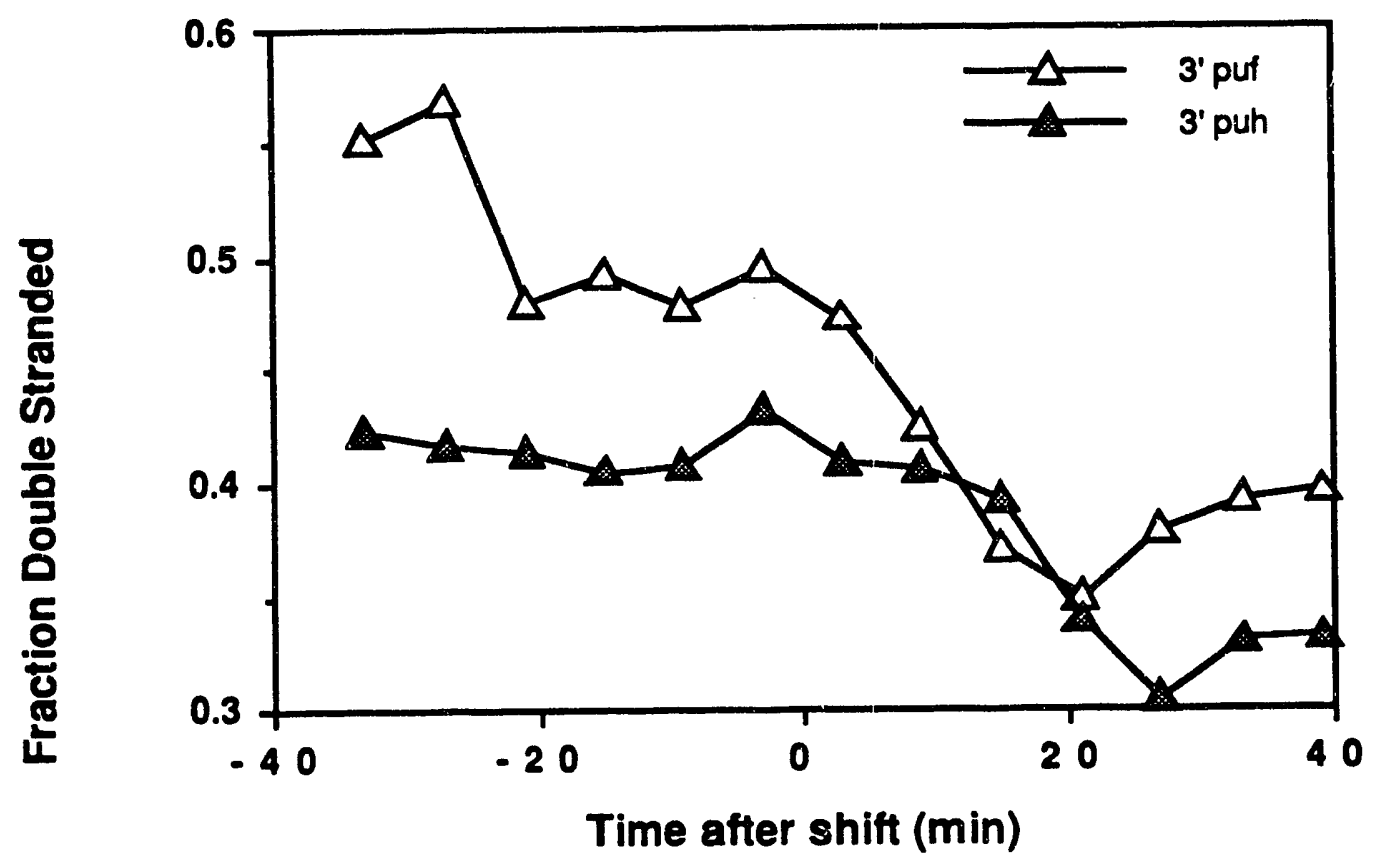

Fig 3.9. TMP reactivity downstream from the reaction center genes. The 3' puf region represents a $4.0 \mathrm{~kb}$ Apal fragment immediately downstream from the puf operon. The 3' puh fragment is a $2.0 \mathrm{~kb}$ EcoRI fragment downstream from the $p u h A$ gene. 
restore supercoils lags behind the rate of rotation of the DNA, then the steady state superhelicity downstream from heavily transcribed genes would be expected to decrease.

Within the puf operon and immediately upstream from the promoter, the pattern of TMP reactivity is quite different (Fig 3.10). The $2.65 \mathrm{~kb}$ Apal fragment upstream from puf has a relatively constant reactivity toward TMP. There may be a transient increase in crosslinking during the first $10 \mathrm{~min}$ after the shift. An increase in negative supercoiling upstream from a gene is predicted by the twin domain model if topoisomerase I, which relaxes the negative supercoils generated by transcription, is insufficient in keeping up with the rotation of the DNA. The $2.8 \mathrm{~kb}$ Apal fragment containing the puf operon shows no clear trend for TMP reactivity in either direction. The scatter in the data may reflect changes in protein coverage, primarily by RNA polymerase, upon the induction of transcription.

Farther upstream from both puf and puh, the reactivity of the DNA again decreases after the shift (Fig 3.11). The $4.6 \mathrm{~kb}$ ApaI fragment lies $2.5 \mathrm{~kb}$ upstream from the expected start site of the puhA operon. Similarly, the $5.0 \mathrm{~kb} \mathrm{EcoH}$ fragment extends substantially upstream from the promoter for the puf region. The possible significance of the decrease in psoralen reactivity for these fragments is examined in the Discussion section in light of the known transcriptional orientation of the genes in this region. 
Fig 3.10 TMP Reactivity Within and Upstream of puf

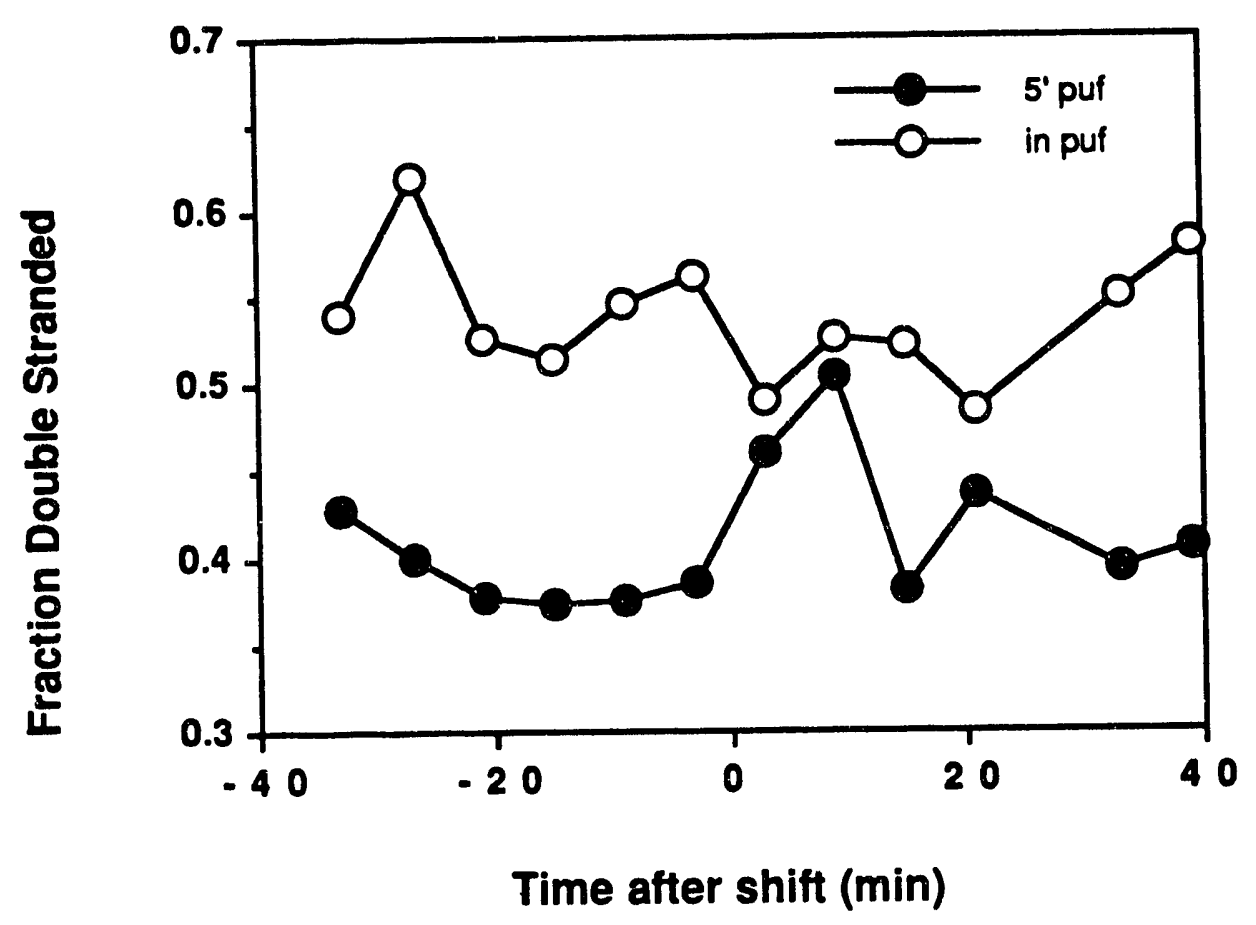

Fig 3.10. TMP reactivity within and upstream from the $p u f$ operon. The puf operon is almost entirely contained on the $2.8 \mathrm{~kb}$ Apal fragment sahown by the open circles (all but $165 \mathrm{bp}$ of the coding region are on this fragment). The upstream region is shown by the darkened circles and represents a $2.65 \mathrm{~kb}$ ApaI fragment which begins $1 \mathrm{~kb}$ upstream from puf. 
Fig 3.11 TMP Reactivity in bch Genes Upstream of puf and puh

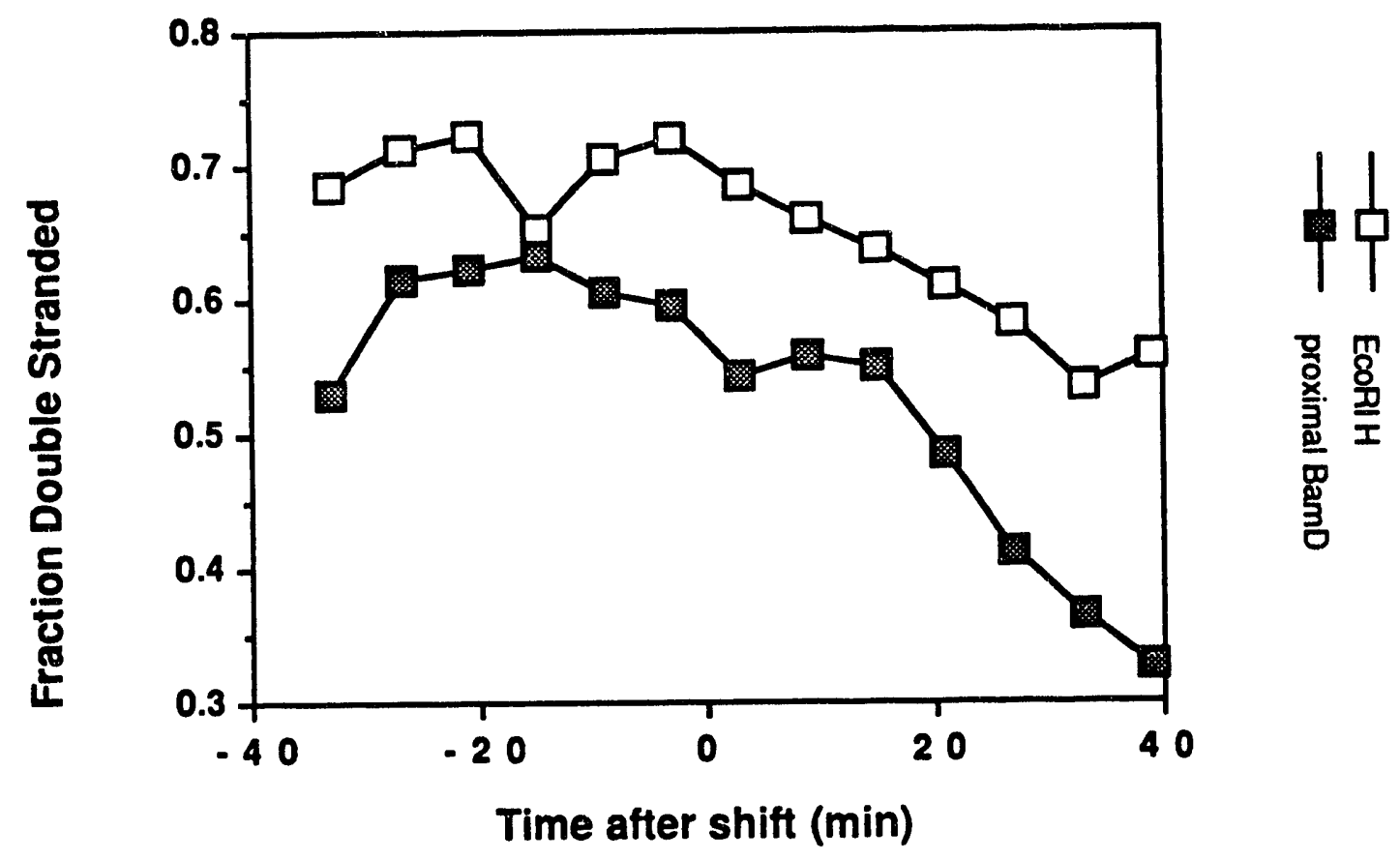

Fig 3.11. The TMP reactivity in $b c h$ coding regions farther upstream from the puf and puh operons. The EcoRI-H fragment contains the $b c h C$ and $b c h A$ genes and begins $0.8 \mathrm{~kb}$ upstream from puf. It is a $5.0 \mathrm{~kb}$ fragment upstream from puf. The proximal BamHI-D probe is a $4.6 \mathrm{~kb}$ Apal fragment in the portion of BamHI-D nearest to the $p u h$ coding region. It begins $2.7 \mathrm{~kb}$ upstream from the puh coding region. 


\section{Discussion}

The utility of the psoralen assay. A novel method for detecting changes in the superhelicity of specific sequences in the bacterial chromosome has been described. The ability to detect changes in superhelicity in local regions allows for a direct appraisal of some long standing questions about the relationship between DNA supercoiling, chromosome structure, and transcriptional activation of genes. The bacterial chromosome has been shown, both in vivo and in vitro, to be composed of topologically independent domains of torsionally strained DNA (Sinden and Pettijohn, 1981; Worcel and Burgi, 1972). Complete relaxation of $E$. coli chromosomal supercoils by DNase I in vitro or by $60 \mathrm{Co} \gamma$-irradiation in vivo indicates that there are approximately 40 of these domains. The number of domains correlates well with the number of chromosomal cleavages induced by treatment of $E$. coli with the gyrase inhibitor oxolinic acid (Snyder and Drlica, 1979). It is intriguing to postulate that the superhelicity of some domains might be maintained at a different level from that of other domains (Higgins et al., 1988; Sinden and Pettijohn, 1981). It has been suggested that differential superhelicity among domains might be important in regulating transcription (Sinden et al., 1980; Worcel and Burgi, 1972), especially since the utilization of a variety of promoters by RNA polymerase has been shown to be a function of the superhelical density of the DNA template (reviewed in Drlica, 1984).

Previous studies involving superhelicity have been performed either on total chromosomal DNA (Drlica and Snyder, 1978; Sinden et al., 1980) or on plasmids (see for example, Lockshon and Morris, 
1983). The former work has provided important information about whole chromosome structure but has not addressed questions concerning specific regions of the chromosome. The latter studies have utilized the exquisite sensitivity of gel systems which can resolve individual topoisomers and provide exact information on the linking number of plasmids. However, studies of bacterial strains with mutations in one of the topoisomerase genes demonstrate that the same mutation can have different effects on gene expression depending on whether the gene of interest is carried on a plasmid or on the chromosome. For instance, mutations in the topA gene of $S$. typhimurium can suppress the leu-500 promoter mutation when the leu-500 promoter is found on the chromosome but not when it is cloned into a plasmid (Richardson et al., 1988). Similarly, topA missense mutations in $S$. typhimurium increase both plasmid superhelicity and expression from the chromosomal proU locus (Higgins et al., 1988). In contrast, top $A$ deletions, which also increase plasmid superhelicity, fail to induce expression of chromosomally encoded proU (Higgins et al., 1988).

Changes in plasmid superhelicity are not always comparable to simultaneous alterations in the chromosome. Gyrase inhibition in $E$. coli by coumarin drugs relaxes the chromosome within $30 \mathrm{~min}$, but the same treatment causes pBR322 to become positively supercoiled (Drlica and Snyder, 1978; Lockshon and Morris, 1983). Similarly, in a $\triangle$ topA gyrB double mutant of $E$. coli, the chromosome is less negatively supercoiled than wild-type; but pBR322 from the same strain is significantly more underwound than plasmids isolated from wild type, with negative superhelicity exceeding that which can be 
produced in vitro by purified gyrase (Pruss et al., 1982; Pruss, 1985). These plasmid anomalies have recently been explained as transcriptionally induced effects caused by the inactivation of topoisomerase proteins (Wang and Liu, 1987; Wu et al., 1988; Brill and Sternglanz, 1988). For some purposes, such as the study of topological questions involving DNA replication or chromosomal domain structure, the TMP assay described here provides a useful alternative to studies on plasmids.

We can estimate the maximum possible change in chromosomal supercoiling which might escape detection by our assay. Relaxation of the chromosome by novobiocin in $R$. capsulatus is complete in about $20 \mathrm{~min}$, as measured by the TMP assay, and changes in superhelicity can be consistently detected at much shorter times when the change in TMP reactivity is small (Fig 3.5). We can reliably detect a change in the rate of cross-linking which is $15 \%$ of the maximal change caused by novobiocin. This limit is greater than the $5 \%$ random variation observed between aerobic and anaerobic cultures in Fig 3.7. Sinden et al. (1980) have shown that the rate of TMP photoreaction is linearly proportional to superhelical density over a range comparable to that found in vivo. Thus a fifteen percent threshold in crosslinking rates would represent a maximal undetectable change of supercoiling in vivo of fifteen percent.

Bliska and Cozzarelli (1987) have recently demonstrated, using a recombinational assay, that $60 \%$ of the supercoils in vivo are probably restrained by interactions with proteins and other cellular components. They estimate that the unrestrained superhelical 
density in vivo is about -0.02 . This number agrees with earlier estimates, by Pettijohn and Pfenniger (1980), that about half of the in vivo supercoils are restrained. Since intercalators are sensitive to unrestrained supercoils but not to DNA wrapped by protein, a $15 \%$ threshold in detection of chromosomal supercoiling translates to a maximum undetectable difference in unrestrained superhelical density of \pm 0.003 .

Stable changes in unrestrained DNA supercoiling do not regulate anaerobic gene expression. Yamamoto and Droffner (1985) suggest that the transition from an aerobic to an anaerobic environment results in gross changes in chromosomal superhelicity, which then activate expression of a large number of genes. This model has received wide attention in the scientific literature, since a true physiological role for chromosomal supercoiling as a mechanism to regulate gene expression has not yet been conclusively demonstrated.

One can argue instead, based on evidence in the literature, that the bacterial cell has evolved a homeostatic mechanism for maintaining relatively constant chromosomal superhelicity (Menzel and Gellert, 1983). Transcription of $g y r A$ and $g y r B$ is stimulated by relaxation of DNA (Menzel and Gellert, 1983; Menzel and Gellert, 1987a; Menzel and Gellert, 1987b) whereas transcription of topA is activated by increased negative superhelicity of the DNA template (Tse-Dinh, 1985). This regulation is exactly that which would be required for maintaining homeostasis. In addition, the substrate affinities and turnover rates of the two topoisomerases are 
dependent on the superhelicity of the DNA target, with gyrase binding best to relaxed DNA molecules (Gellert et al., 1976), while topoisomerase I prefers negatively supercoiled DNA (Wang, 1971). This biochemical evidence indicates that the topoisomerases might be distributed over the chromosome so as to minimize local differences in DNA superhelicity.

The data presented here are the first direct test of the Yamamoto-Droffner model to monitor for changes in chromosomal supercoiling upon a shift to anaerobic conditions. Previous studies have examined the effects of topoisomerase mutations or gyrase inhibition on bacterial physiology. These experiments are complicated by the many pleiotropic effects of topoisomerase inactivation. The method described here is sensitive to local changes in DNA supercoiling which might be missed by other techniques. The psoralen assay accurately monitors stable changes in chromosomal supercoiling upon inhibition of DNA gyrase with novobiocin. Yet we observe no change in psoralen reactivity upon a shift from aerobic to anaerobic growth. We conclude, therefore, that DNA supercoiling does not play a regulatory role in the expression of genes for anaerobic metabolism.

What then is the role of DNA gyrase in the transcription of the puf and puh operons? The loss of mRNAs from these loci after treatment with gyrase inhibitors could be an indirect result of inhibitor treatment. Gyrase is clearly an essential enzyme for DNA metabolism, and loss of gyrase activity has numerous and complex effects on the cell which may not directly reflect its immediate role in bacterial physiology (Kreuzer and Cozzarelli, 1979). On the other 
hand, evidence is accumulating which indicates that gyrase may act as a swivel in the transcription of strongly expressed genes and so may be essential to the expression of the puf and puh operons (see below).

The role of transcriptional supercoiling and DNA gyrase in the expression of the puf and puh operons. The initial experiments described above were designed to detect stable changes in chromosomal superhelicity due to changes in gyrase activity, in accord with the Yamamoto and Droffner model. These experiments, in which irradiations are carried out on ice after an extensive equilibration period, might not be sensitive to transient changes in superhelicity induced by transcription, since RNA polymerase is itself inactive under these conditions. The final experiments, using actively metabolizing cells irradiated in flow, provides support for the transcriptional model (Figs. 3.9-3.11). These data are summarized schematically in Fig 3.12.

There is a dramatic decrease in TMP reactivity immediately downstream from the strongly expressed genes for the photosynthetic reaction center subunits (Fig 3.9 and 3.12). The model for transcriptional supercoiling predicts exactly this result: downstream from a heavily transcribed gene, the positive superturns introduced by transcription would decrease the steady state superhelicity. That region could be demarcated by some topological barrier which prevents the diffusion of supercoils along the DNA template or it could be bounded by a site or sites where gyrase is actively removing the positive supercoils. Overwinding of the DNA in the downstream region 
Fig 3.12. Schematic presentation of the results of the flow irradiation on shifted cultures of $R$. capsualtus. The reaction center genes (puf and $p u h)$ are shown by stippled rectangles. Downstream from these genes, which are heavily transcribed, the psoralen reactivity decreases during the shift to anaerobic growth conditions. Within each reaction center operon, the psoralen reactivity is roughly constant, denoted by \pm . The Apal fragment immediately upstream from puf also shows little or no change in reactivity. Finally, farther upstream from both operons, the reactivity decreases during the shift. Note the tight clustering of genes within this region and the parallel orientation of transcription. Restriction sites for EcoRI (circles) and Apal (filled diamonds) are shown. 
Fig 3.12A

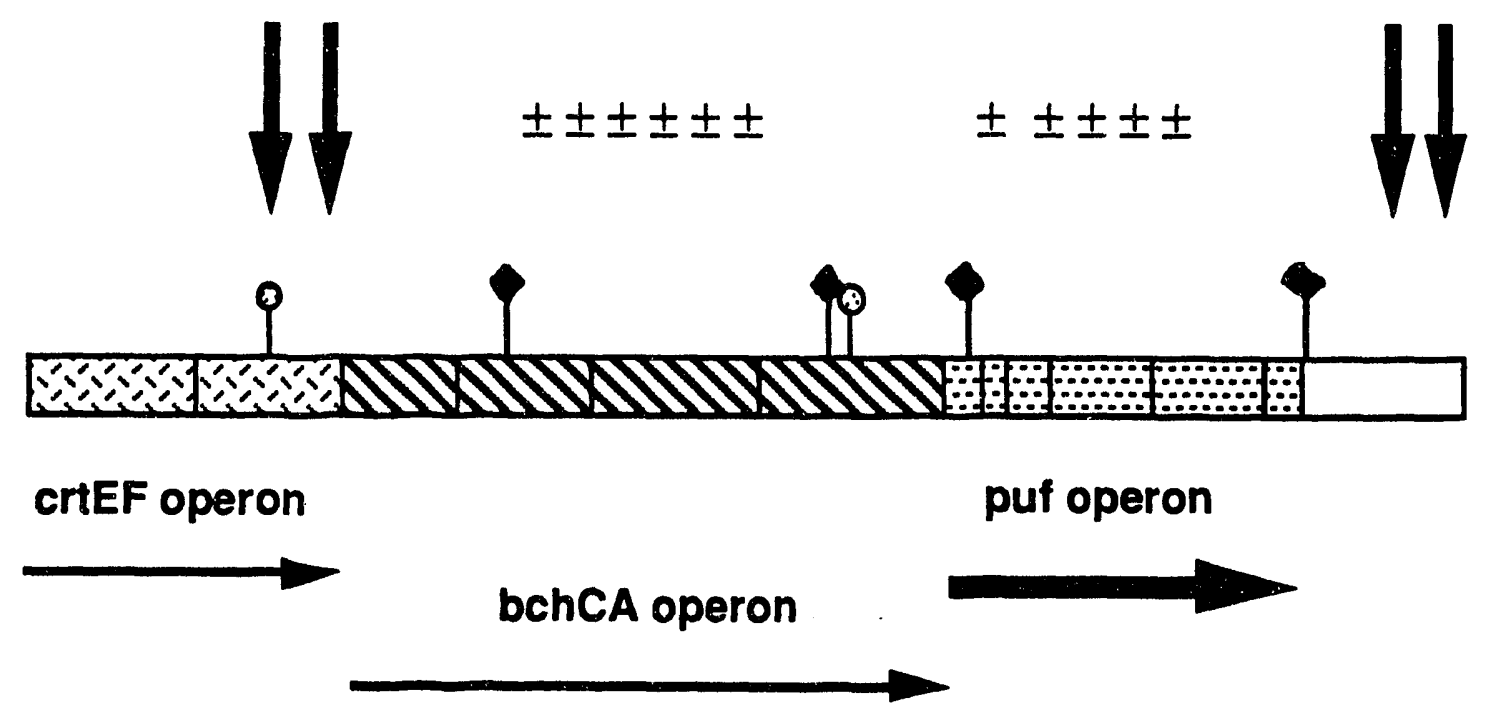

Fig 3.12B
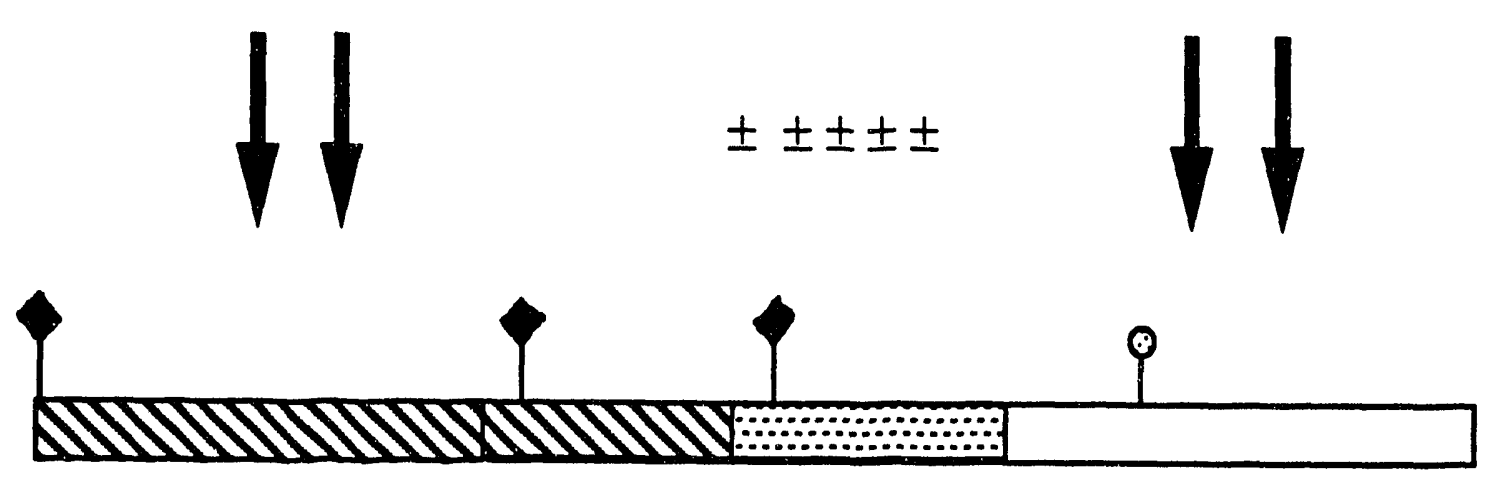

puh operon

bch L operon 
would diminish the binding constant of TMP and thus the yield of crosslinked DNA in the psoralen assay.

The immediately adjacent fragments, which contain the puf and puh genes, do not undergo this decrease in reactivity (Fig 3.10 and 3.12). Within the transcribed region, the twin domain model leads to the prediction of no net change in superhelicity, because for every polymerase which introduces negative supercoils, there is another polymerase in its wake putting in positive supercoils. Assuming that the polymerase itself does not alter the pattern of psoralen reactivity by protecting the DNA, there should be no net change in psoralen reactivity within the gene. This is what we observe in the flow experiment (Fig 3.10).

In the regions upstream from the puf and $p u h$ coperons, the data are more complicated. For a $2.6 \mathrm{~kb}$ Apal fragment, just upstream from the puf promoter (Adams et al., 1989; Bauer et al., 1988), TMP reactivity is almost constant, with possibly a small increase in the first $10 \mathrm{~min}$ after the shift. Farther upstream, however, the pattern of psoralen reactivity is similar to that for the DNA downstream from the reaction center genes: after the shift, TMP reactivity decreases significantly, reaching a minimum after 30 min (Fig. 3.11). This phenomenon may be due to the fact that many genes in the photosynthesis cluster are being activated simultaneously. Genetic mapping (Young et al., 1989; Yang and Bauer, 1990), analysis of mRNA (Wellington and Beatty, 1989), and sequence data (D. Burke-Agüerro, M. Alberti, and J. Hearst) indicate that genes for bacteriochlorophyll biosynthesis, located immediately upstream from both $p u f$ and $p u h$, are transcribed in the same 
direction as the genes for the reaction center (Fig 3.12).

Furthermore, genetic analysis (Young et al., 1989; Yang and Bauer, 1990) indicates that these bacteriochlorophyll genes are part of two superoperons extending many kilobases upstream from $p u f$ and puh, which allow for cotranscription of the pigment biosynthesis enzymes and the pigment-binding proteins. Transcription of these superoperons may induce positive supercoils upstream of puf and puh which decreases psoralen reactivity in this region.

In conclusion, the data presented here support an alternative hypothesis to that advanced by Yamamoto and Droffner for the dependence of transcription of photosynthesis genes on DNA gyrase. The interpretation of these results is summarized in Fig 3.13. Induction of photosynthesis involves a substantial commitment by the bacterial cell to the production of membrane-bound reaction center and light harvesting pigment-protein complexes, which are encoded by the puf and puh operons. Transcription at these loci increases significantly upon a shift to anaerobiosis. The torque exerted on the DNA by transcription will therefore also increase. It is also important that the nascent proteins are probably inserted into the photosynthetic membrane as they are being synthesized. Membrane insertion may serve to anchor the transcription ensemble more firmly and prevent it from rotating freely around the DNA template. Under these conditions, gyrase and topoisomerase I would be required as swivels to relieve the overand underwinding of the chromosome. Thus, instead of activating transcription by altering DNA supercoiling, gyrase is required as a consequence of transcription. 
Fig 3.13. A model for the requirement of DNA gyrase in the expression of genes for anaerobic photosynthesis in Rhodobacter capsulatus. Induction of anaerobic metabolism requires the production of a photosynthetic membrane, with its constituent membrane-bound reaction center and light harvesting complexes. Transcription of these genes results in the simultaneous translation and insertion of the nascent polypeptides into the developing intracytoplasmic membrane. This membrane insertion can serve to anchor the transcription ensemble and drive rotation of the DNA. Topoisomerase I and DNA gyrase are essential swivels to relieve this supercoiling on the chromosome. Inhibition of gyrase would halt transcription of the reaction center genes, as observed by $\mathrm{Zhu}$ and Hearst (1989). 
Fig 3.13
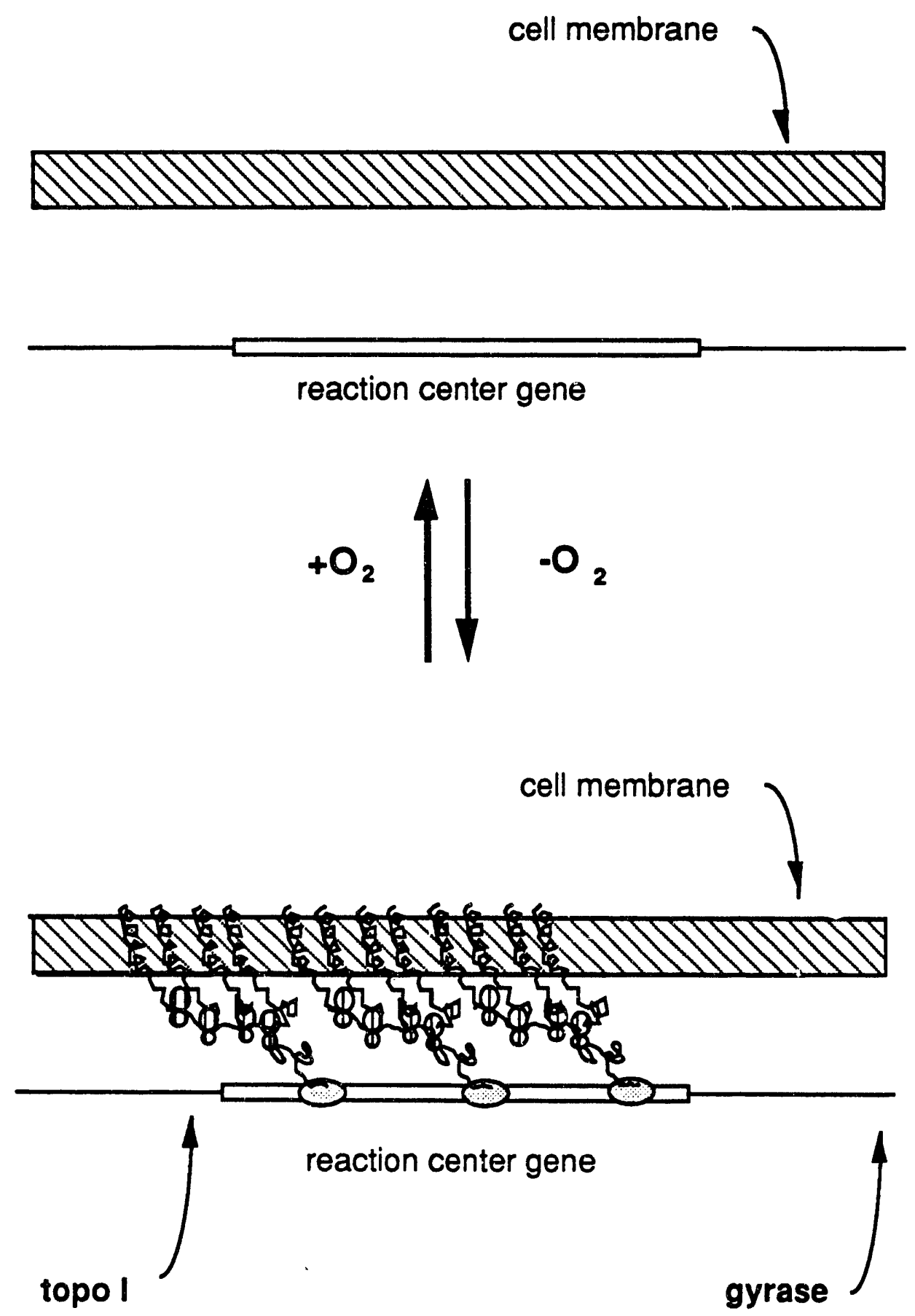
Uncertainties of the twin domain model. On a large DNA molecule such as the bacterial chromosome, it is not clear how large a region of DNA should be affected by transcription at a given locus. The barriers to diffusion of supercoils, if any, are not clearly understood. Interactions of the chromosome with the inner membrane (Ogden et al., 1988; Liu and Wang, 1987) or attachment to large nucleo-protein structures could lead to the formation of topological anchors which limit the diffusion of supercoils and define precise topological domains. Topoisomerase I and gyrase could act at defined sites (Franco and Drlica, 1988) which might also delineate clear topological boundaries. In addition, the physical parameters which influence the efficiency of transcriptionally induced supercoiling, including the length of the mRNA, the frequency of initiation of transcription, the rate of transcription elongation, the influence of neighboring genes, the coupling of translation to transcription (Koo, et al. 1990), and the importance of membrane insertion of nascent polypeptides (Lodge et al., 1989), are not well understood. Finally, the ability of thie topoisomerases themselves to compensate for transcriptional supercoils is unknown. These uncertainties are discussed further in Chapter 4, and experiments are presented to achieve a better understanding of transcriptional supercoils. 


\section{Chapter 4}

\section{A Model System for Investigating Transcriptional Supercoiling}

\section{Introduction}

Movement of a macromolecular complex, such as RNA polymerase, along a DNA can drive rotational motion of the DNA (Liu and Wang, 1987). If the DNA is topologically anchored, generation of supercoils by DNA tracking processes may lead to dynamic variation of superhelix density over the time range of seconds to minutes. The goal of the experiments described in this chapter is to develop a model system for the study of transcriptional supercoiling in vitro and in vivo in order to understand some of the physical mechanisms and biological consequences of this phenomenon. Furthermore, I would like to relate the results of these experiments to the kinetic data presented in Chapter 3 which indicates that transcriptional supercoils are generated on the bacterial chromosome.

Experimental background to the twin domain model. Several early observations were critical to the development of the idea that RNA polymerase can drive rotational motion of the template. Lockshon and Morris (1983) initially observed that inhibition of DNA gyrase produces positive supercoils in plasmid pBR322 in vivo (Lockshon and Morris, 1983). Complete overwinding of the plasmids in a bacterial population takes about $30 \mathrm{~min}$. At earlier times, the topoisomer distribution is highly heterogeneous, containing both 
overwound and underwound plasmid species. In a parallel observation, Pruss and Drlica (1986) reported that the linking number distribution of plasmid pBR322 in $\triangle t o p A$ strains of $E$. coli was heterogeneous and skewed towards highly negative superhelix densities (Pruss and Drlica, 1986). They further showed that this change in linking number was dependent on transcription of the tet gene on the plasmid.

These observations were unified by the elaboration of the twin domain model by Liu and Wang (1987) (Fig 1.3). The twin domain model postulates that the frictional drag on the transcription ensemble can be large compared to that generated by rotation of the double helix with its comparatively small axial radius of gyration. Under circumstances where the DNA is anchored, the positive supercoils formed ahead and the negative supercoils formed behind the tracking complex can be trapped in localized domains. The plasmid pBR322 allows for experimental verification of this idea, since simultaneous transcription of opposing tet and amp genes on a single molecule creates topological domains (Fig 4.1). These ideas were elegantly elaborated in an experimental paper by Liu, Wang, and co-workers (Wu et al., 1988). Two other papers established the same phenomenon in yeast (Brill and Sternglanz, 1988; Giaever and Wang, 1988).

A potentially important consequence of transcriptional supercoiling is that two genes can regulate one another simply on the basis of their orientation and proximity (Pruss and Drlica, 1989). Transcription at one locus could activate a neighboring gene, depending on its sensitivity to DNA super ooiling. It is possible, 
Fig 4.1. Transcription of tet and amp on pBR322

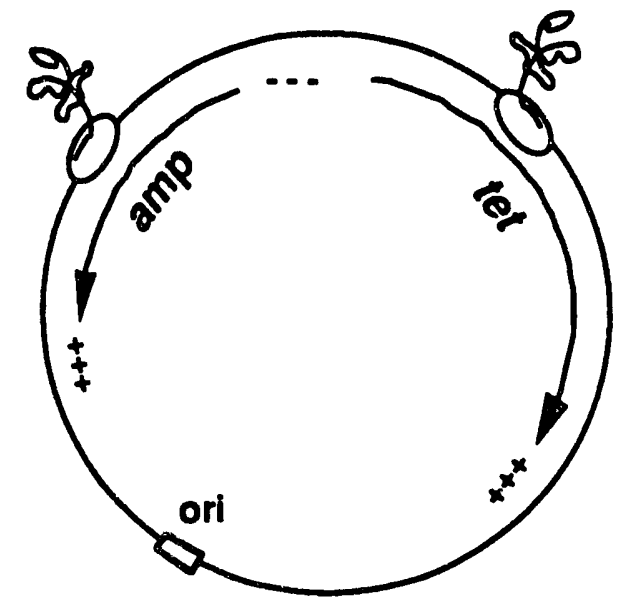

Fig 4.1. The arrangement of $a m p$ and tet genes on pBR322. The orientation of the genes for $\beta$-lactamase $(a m p)$ and tetracycline resistance (tet) are shown, as is the location of the origin of replication. Also depicted in cartoon form are two transcribing RNA polymerases and their nascent mRNA. The positively supercoiled domain is shown by +++ and the negative domain by - - - 
furthermore, that the build-up of positive supercoils downstream from RNA polymerase could limit the rate of elongation by causing the transcription complex to rotate around the DNA. Transcriptional supercoils could therefore limit gene expression.

Another implication of the twin domain model relates to the function of topoisomerases in the cell. Since transcription is ubiquitous, one could postulate that the unrestrained supercoils observed in bacterial cells are generated principally by $\mathrm{m}$ : tracking processes (Liu and Wang, 1987). An essential role of the topoisomerases would therefore be to compensate for the rapid creation of positive and negative sul ccoils by transcription. This point of view represents a departure from prevailing opinion which holds that the level of supercoiling in vivo is determined solely by a dynamic competition between topoisomerase I and gyrase (Gellert, 1981; Wang, 1985).

Data from eukaryotic cells already strongly suggest that transcription elongation at the rDNA locus is coupled to the swivel action of topoisom rases. In yeast, the approximately 200 copies of rDNA are arrayed in tandem on a single chromosome (Brewer, 1988). Transcription at this locus is among the most active in the cell, since ribosomes constitute a major fraction of the cellular mass. Various lines of evidence suggest that topoisomerase I may serve as a swivel to remove the supercoils generated by transcription. The major site for immunostaining with anti-topoisomerase $I$ antibodies is the nucleolus, the organelle in which rRNA is produced (Müller et al., 1985). Elongation of rDNA transcription is limited when topoisomerases $I$ and $i$ are inactivated (Brill and Sternglanz, 1988). 
Other studies suggest that, in the absence of topoisomerases, the build-up of torsional stress at the rDNA locus drastically increases the recombination frequency in this region. Recombination of rDNA is stimulated 50 - to 200 -fold when both topoisomerases are inactivated by mutations, but recombination elsewhere in the genome is unaffected (Christman et al., 1988). In a similar genetic background, rDNA sequences are reversibly excised from the chromosome and exist in an episomal state (Kim and Wang, 1989). Taken together, these results are suggestive of a strong link between transcription and supercoiling, which can stimulate recombination, on the chromosome of yeast.

It is significant that, in all of the experimental papers cited above, the protocol for observing transcriptional supercoils involves inhibiting either one (in bacteria) or both (in yeast) of the topoisomerases. This implies that, under normal circumstances, either the topoisomerases cope efficiently with transcriptional supercoils or that RNA polymerase drives rotation of DNA very inefficiently. One of the motivations for the experiments described here is to develop a model system to optimize the efficiency of transcriptional supercoiling to determine whether there are biological effects in the presence of functional topoisomerases (see below).

Recently, Rahmouni and Wells (1989) have demonstrated the persistence of localized differences in superc iling on plasmid pBR322 without inhibiting topoisomerases (Rahmouni and Wells, 1989). Their assay involves the sensitive detection of Z-DNA tracts using an osmium tetroxide reaction which specifically modifies left- 
handed DNA. They show that stretches of $d(G C)$ only 12 base pairs long can form Z-DNA upstream but not downstream from transcription on pBR322. The formation of Z-DNA is driven by the free energy of negative supercoiling. By calibrating in vitro the superhelical densities at which the $B$ to $Z$ transition occurs, Rahmouni and Wells place upper and lower bounds on the unrestrained negative superhelix densities achieved locally on the plasmid. The localized superhelix density on pBR322 can be $\leq$ 0.038 upstream from transcription and $\geq-0.021$ downstream from transcription.

The optimization of transcriptional supercoiling. The osmium labeling technique employed by Rahmouni and Wells is extremely sensitive. The adduct formed by reaction with thymidine residues in a Z-DNA tract blocks primer extension by the Klenow fragment of DNA polymerase I. Since the products of primer extension are labelled with 32P, the presence of Z-DNA stretches can be detected even if only a minute fraction of the plasmids has localized domains of supercoiling. In order to assess the generality of transcriptional supercoils, the process of driven rotation of the DNA by RNA polymerase must be optimized.

There are several reasons to believe that transcriptional supercoiling on pBR322 may be less than optimally efficient. First, the formation of topological domains on $\mathrm{pBR} 322$, according to the twin domain model, requires simultaneous transcription of both the tet and amp genes on a single molecule. If the promoters initiate transcription with independent probability, then the likelihood that 
both genes are being transcribed is simply the product of the probabilities of occupancy of either gene. The observations by both Pruss and Drlica (1986) and Lockshon and Morris (1983) of a heterogeneous distribution of topoisomers suggest that at any one time, only a fraction of the plasmid population is being driven by transcription. One way to optimize transcriptional supercoiling would be to use the strongest possible promoters in place of those normally found on pBR322.

Another requirement for transcriptional supercoiling is the production of an RNA transcript of significant length. In vitro, the generation of supercoils by T7 RNA polymerase occurs only after the production of an RNA species which is longer than 5 kilobases; addition of RNase to the transcription reaction effectively inhibits transcriptional supercoiling (Tsao et al., 1989). In $\Delta t o p A$ strains, deletion of internal portions of the tet gene can abolish the production of the highly underwound species of pBR322 (Lodge et al., 1989). These observations underscore the importance of the nascent RNA in developing sufficient frictional drag to drive the DNA. In fact, it may be that only towards the later stages of transcription, when a sizeable RNA chain has been synthesized, is there significant torque applied to the double helix. This suggests that not only must both genes on pBR322 be transcribed, but RNA polymerases may need to be simultaneously in the later stages of copying the opposed tet and amp genes, thus further reducing the probability that any given molecule is being actively supercoiled by transcription. A second way, therefore, of optimizing transcriptional supercoiling would be to fuse long operons behind strong promoters. 
Such a construct would optimize the hydrodynamic drag on the transcription ensemble by maximizing the length of the transcript.

Finally, there is some suggestion that coupled transcription and translation increases the efficiency of transcriptional supercoiling in bacteria (Liu and Wang, 1987; Lodge et al., 1989). This effect is also likely due to the requirement for a large drag on the transcription ensemble. Ribosomes, the largest non-organellar structures in cells, can contribute significant mas to the transcription ensemble. In the appropriate situations, simultaneous translation and insertion of nascent proteins into the cell membrane can further increase the efficiency of transcriptional supercoiling by firmly anchoring the RNA polymerase complex (Liu and Wang, 1987; Lodge et al., 1989).

Qutline of the chapter. The material presented here represents a summary of some work in progress. I describe the construction of plasmids designed with the criterion of optimizing transcriptional supercoiling. An $E$. coli $\mathrm{K}-12$ strain has been constructed which is both recombination deficient and permeable to drugs which inhibit DNA gyrase. After activation of transcription and inhibition of DNA gyrase, these plasmid constructs form a band at high density in $\mathrm{CsCl}-\mathrm{EtBr}$ density gradients, consistent with the formation of positively supercoiled DNA. Surprisingly, the presence of this high density band is independent of the orientation of the genes on the plasmid. The consequences of transcriptional supercoiling on the rate of transcription are investigated by assaying for the expression 
of one of the gene products. Finally, a series of future experiments is described for a more complete investigation of this system.

\section{Materials and Methods}

Bacterial Strains. The host strain for transformation of $P_{A 1-04 / 03}$ plasmid constructs was D1210, a laciQ derivative of HB101. E. coli K12 strain D21e7 (proA23, lac-28, tsx-81, trp-30, his-51, rpsL173, $r f a-1, a m p(p 1)$, an outer membrane mutant permeable to small hydrophobic molecules (Havekes et al., 1977) was received from B. Bachman at the E. coli Genetic Stock Center. D21e7DrecA was constructed from D21e7 by $\mathrm{P} 1$ transduction using a phage P1 lysate prepared on strain JC10289 $(\Delta(\operatorname{srl}-$ recA)306) (Csonka and Clark, 1979) which was kindly provided by Ann Templin. P1 transduction was performed according to a standard protocol (Willetts et al., 1969).

Plasmids. Cloning was performed by standard methods as detailed in Sambrook et al. (1989). Ligations were performed in gel using specific fragments by cutting DNA bands out of low melting point gels.

LacZ activity time-courses. LacZ assays were performed essentially according to Miller (1972). Cells at an $\mathrm{OD}_{600}$ of 0.4 to 0.5 were induced with $0.5 \mathrm{mM}$ isopropyl- $\beta$-D-thiogalactoside, IPTG. Aliquots $(1 \mathrm{ml})$ were harvested at intervals and the $O_{600}$ was simultaneously read using a side-arm flask in a Bausch and Lomb Spectronic 20. Aliquots were immediately frozen in liquid nitrogen and stored at - 
$70^{\circ} \mathrm{C}$ for later analysis. Cells stored in this fashion retained full LacZ activity even after several freezing and thawing cycles. Thawing was always performed slowly on ice for 1-2 hr before the assay. The LacZ activity of induced D21e7 $\Delta$ recA cultures was so high that $10 \mu \mathrm{l}$ or less of cells were required per assay. Cells $(100 \mu \mathrm{l})$ were diluted in Z-buffer $(900 \mu \mathrm{l})$ (Miller, 1972), and then the appropriate volume $(20-100 \mu \mathrm{l})$ of the dilution was assayed. At this low number of cells, activities from samples lysed according to the $\mathrm{SDS}-\mathrm{CHCl}_{3}$ protocol were not linear with cell number, presumably due to denaturation of protein by SDS. Therefore, toluene was routinely used to permeabilize cells to the LacZ substrate, o-nitrophenyl- $\beta$ - $D$ thiogaloctoside (Miller, 1972).

$\mathrm{CsCl}-\mathrm{EtBr}$ density gradients. Plasmids pNPO-lactrp5.0 and pNPP-lactrp5.0 are each larger than $17 \mathrm{~kb}$, too big to resolve topoisomer species on gels. In an initial effort to characterize the effect of transcription on superhelix density, CsCl-EtBr density gradients were run on plasmid DNA isolated from $400 \mathrm{ml}$ cultures grown to mid-log phase and treated as described in the text. The gradients were performed as described in Chapter 2 .

\section{Results}

Construction of plasmids for maximizing transcriptional supercoiling.

The principle idea behind the plasmids described here is to construct molecules with strongly expressed genes in opposed orientation which, according to the twin domain model, should maximize the efficiency of transcriptional supercoiling. The 
following criteria were considered: (1) The promoters for transcription initiation should be the strongest possible and should be tightly regulated so that gene expression can be syrichronously activated in a bacterial culture; the topological state of the plasmids can therefore be examined with and without transcription. (2) The operons to be expressed should be as long as possible to maximize the length of the mRNA, and thereby increase the torque on the DNA by the transcription ensemble. (3) The gene products should be easily assayed so that effects on expression can be measured. An appropriate control plasmid, with the operons in parallel rather than opposed orientation, should be constructed.

In selecting operons, the length of the transcribed region was paramount. For this reason, I chose the trp and lac operons from $E$. coli (Beckwith, 1987; Yanofsky and Crawford, 1987) which are each longer than 5 kilobase pairs (Fig 4.2) This compares the the amp and tet genes of $\mathrm{pBR} 322$, both of which are shorter than 1.4 kilobase pairs. In addition, the $\operatorname{lac} Z$ gene product, $\beta$-galactosidase, and the $\operatorname{trp} A B$ gene product, tryptophan synthetase, are easily assayed in crude cellular extracts (Miller, 1972; Smith and Yanofsky, 1962). Normally the trp operon includes an attenuation region which, in the presence of excess tryptophan, causes premature termination of transcription. The attenuator was omitted in the constructs describe below.

The promoter chosen for the plasmids was $P_{A 1-04 / 03}$, a hybrid of the strongly expressed $P_{A 1}$ promoter from phage $T 7$, and the 04/03 lac operator sites (Fig 4.3). This promoter, constructed in the laboratory of Dr. H. Bujard at the Zentrum fïr Molekulare Biologie, 
Fig 4.2A

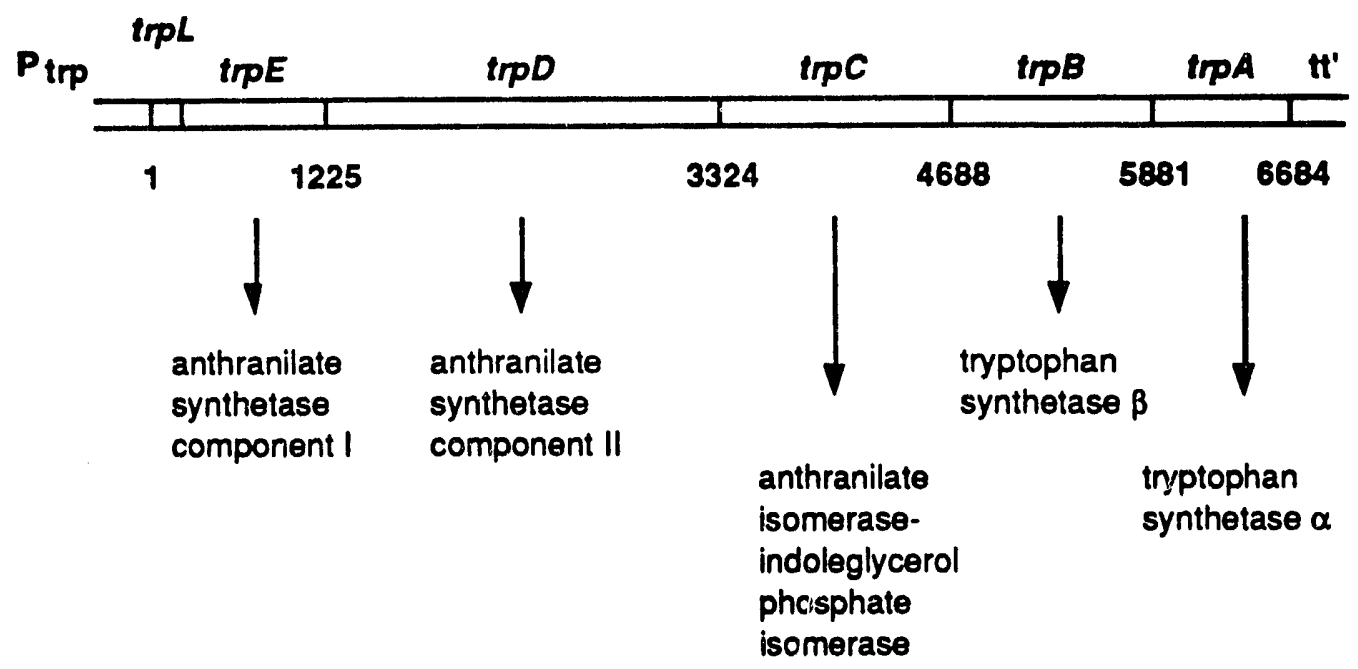

Fig 4.2B

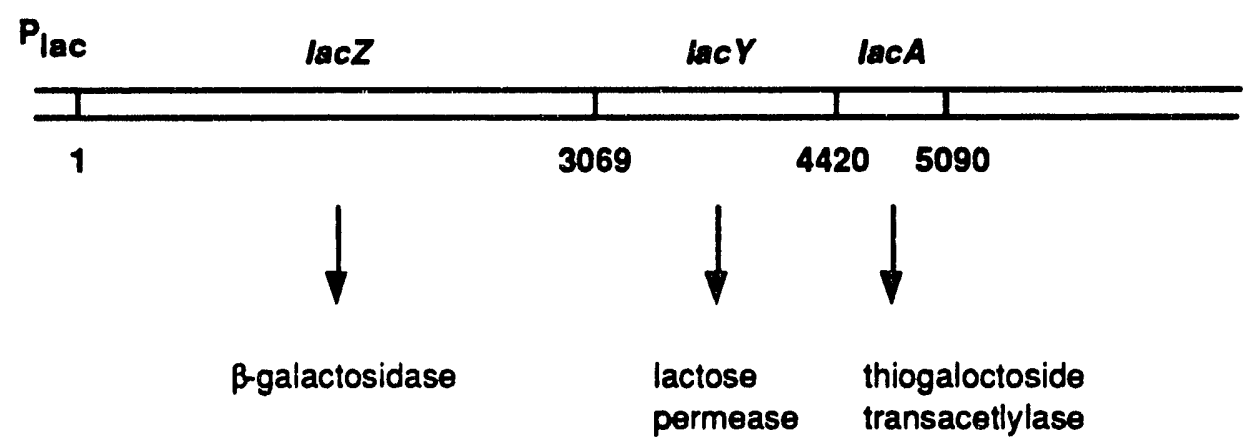

Fig 4.2. The genetic-physical maps of the trp and lac operons. 


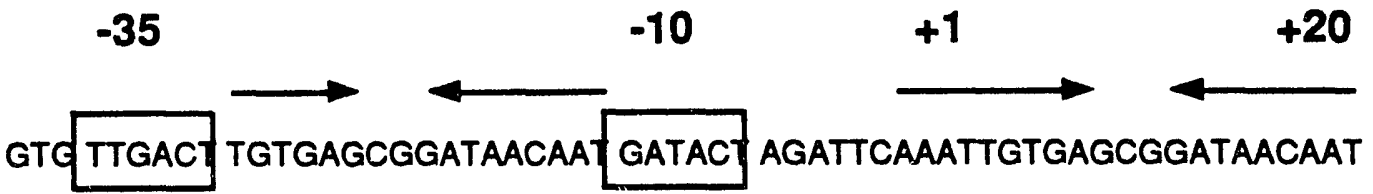

Fig 4.3. The sequence and structure of the PA1-04/03. The -10 and -35 regions of the PA1 promter from phage $\mathrm{T} 7$ are shown in boxes. Arrows represent the 04 and 03 lac repressor binding sites. Nucleotide positions are given relative to the start of transcription at +1 .

Universität Heidelburg, is inducible by the addition of IPTG to a culture, but is repressed in strains which contain laciQ, a constitutive gene for the lac repressor. Transcription from the wild-type $P_{A 1}$ promoter initiates in vivo with nearly maximum efficiency (Deuschle et al., 1986). Rates of transcription are limited, in principle, by the rate of elongation $(\sim 50 \mathrm{bp} / \mathrm{sec})$ and the size of the footprint of the polymerase at the promoter $(\sim 50 \mathrm{bp})$. Deuschle et al. (1986) estimate that the wild-type $P_{A 1}$ promoter achieves a frequency of starts which approaches the limiting frequency of 1 per sec. This rate is comparable to the P1 promoter for the $\operatorname{rrnB}$ operon. Relative to $\mathrm{P}_{\mathrm{bla}}$, the promoter for the amp gene of pBR322, with an activity of $1.0, P_{A 1}$ has an activity of 76 (in $P_{b l a}$ units) (Deuschle et al., 1986). $P_{A 1-04 / 03}$ is diminished somewhat in promoter strength, but still has an activity of $32 \mathrm{P}_{\text {bla }}$ units. A construct incorporating $\mathrm{P}_{\mathrm{A} 1-04 / 03}$ should therefore be significantly 
more efficient in producing transcriptional supercoils than are the tet and amp promoters on $\mathrm{pBR} 322$.

I chose to drive the expression of both trp and lac operons with the same promoter. Using a different promoter for each transcription unit might lead to uncertainty as to the probability of initiation of both operons on a single molecule. With the same promoter, each operon is equally likely to be transcribed. With different promoters, one operon might have an unexpected dominance over the other based, for instance, on a differential sensitivity to transcriptional supercoils.

The steps in cloning the constructs are outlined in Fig 4.4. Into the expression vector pUHE21.2 (from $\mathrm{H}$. Bujard), both trp and lac operons were inserted, resulting in the plasmids pPA1-trp5.0 and pPA1.lac (fig 4.4A). Note that pUHE21.2, the parent vector, includes the transcription termination signal $t_{1}$ from the $\operatorname{rrnB}$ operon. This terminator was retained downstream from trp and $l a c$ in all later constructs. Furthermore, the cat gene, conferring resistance to the antibiotic chloramphenicol, was retained in all subsequent trp constructs. Neither trp (Yanofsky et al., 1981) nor lac (Büchel et al., 1980; Hediger et al., 1985; Kalnins et al., 1983) has Xhol or XbaI

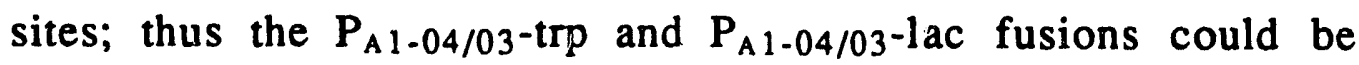
conveniently mobilized into later constructs by cutting at these sites.

The $5.0 \mathrm{~kb}$ HindIII-Sall fragment of the $\operatorname{trp}$ operon in pPA1 trp5.0 was derived from plasmid pBN24 (Schneider, Nichols et al., 1981) as an EcoRI-Sall fragment. The additional EcoRI-HindIII fragment from pBN24 added 29 bp from pBR322. Thus, pPA1.trp5.0 lacks the RBSII ribosome binding site, and translation of trp genes 


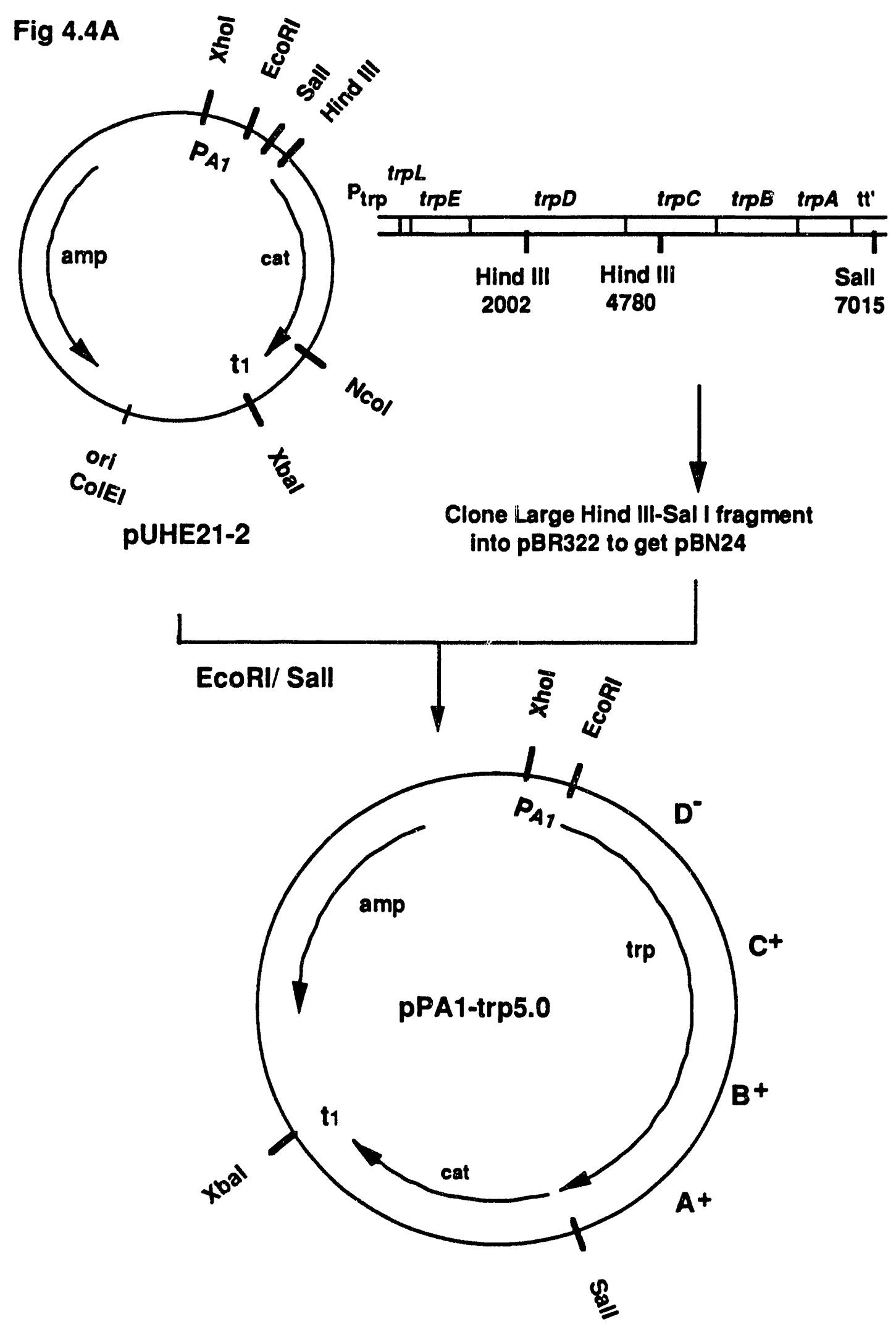

188 
Fig 4.4A (continued)

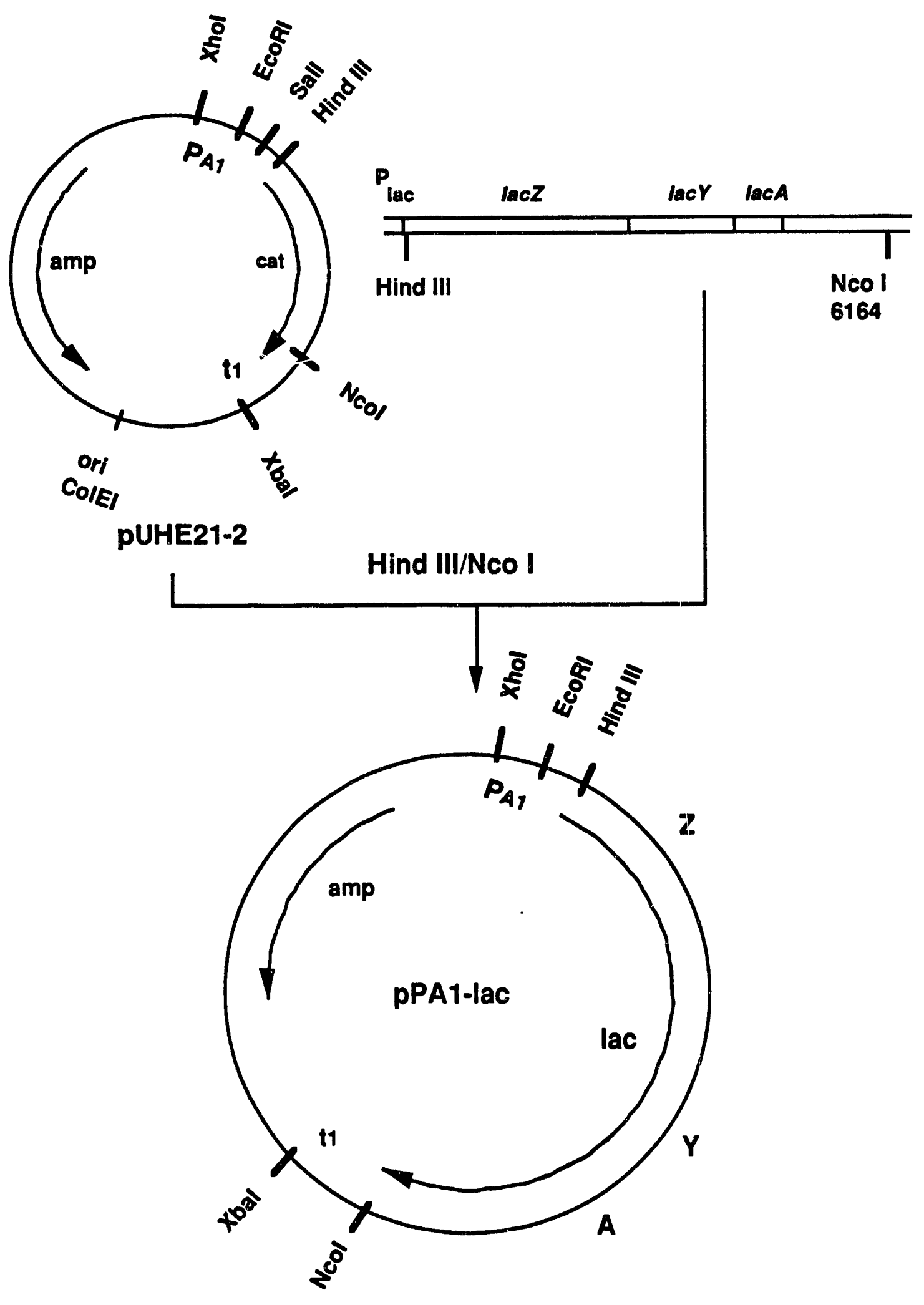


Fig 4.4B. Modification of pBR322
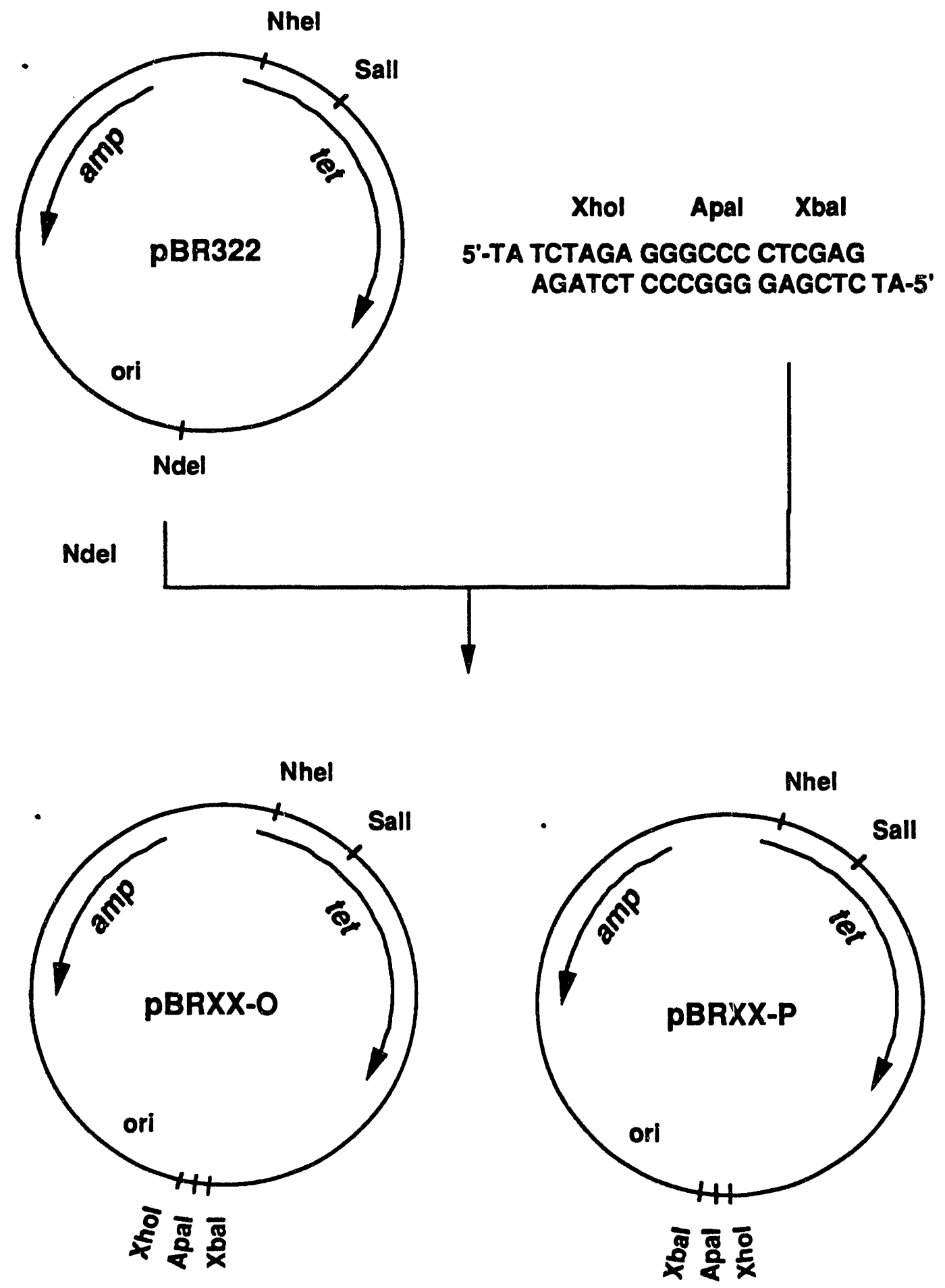
Fig 4.4C
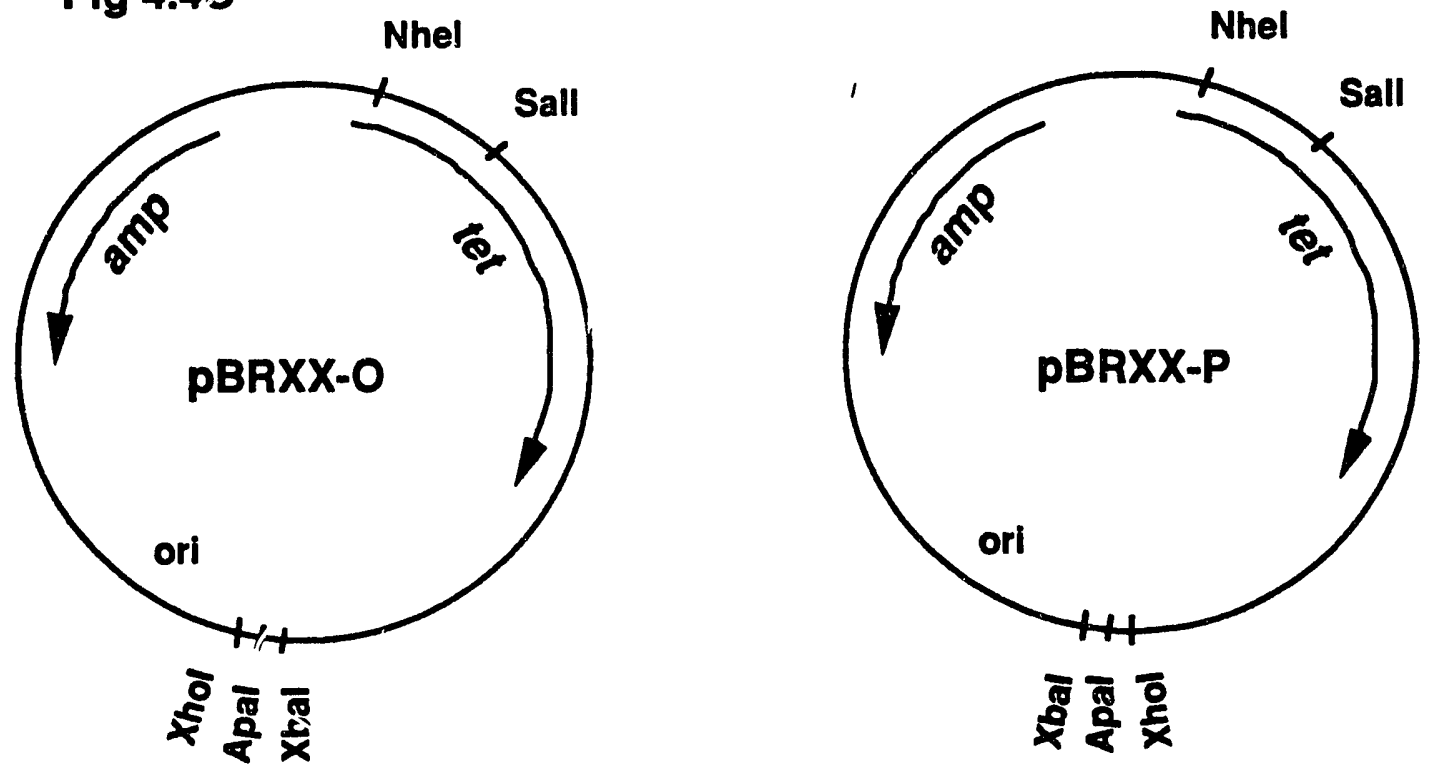

- Cut with Sall and Nhel
- ligate with Xhol/Xbai
fragment from pPA1-lac

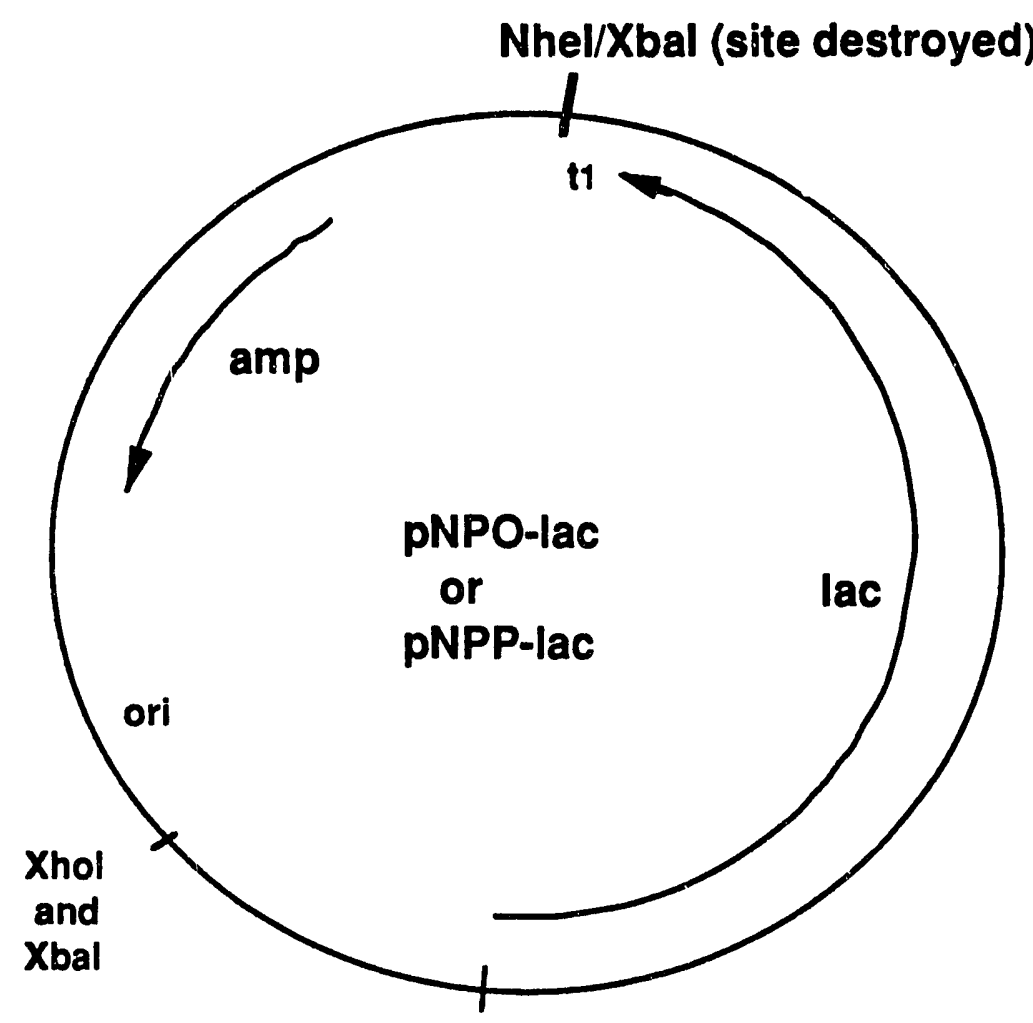

Sall/Xhol (site destroyed) 

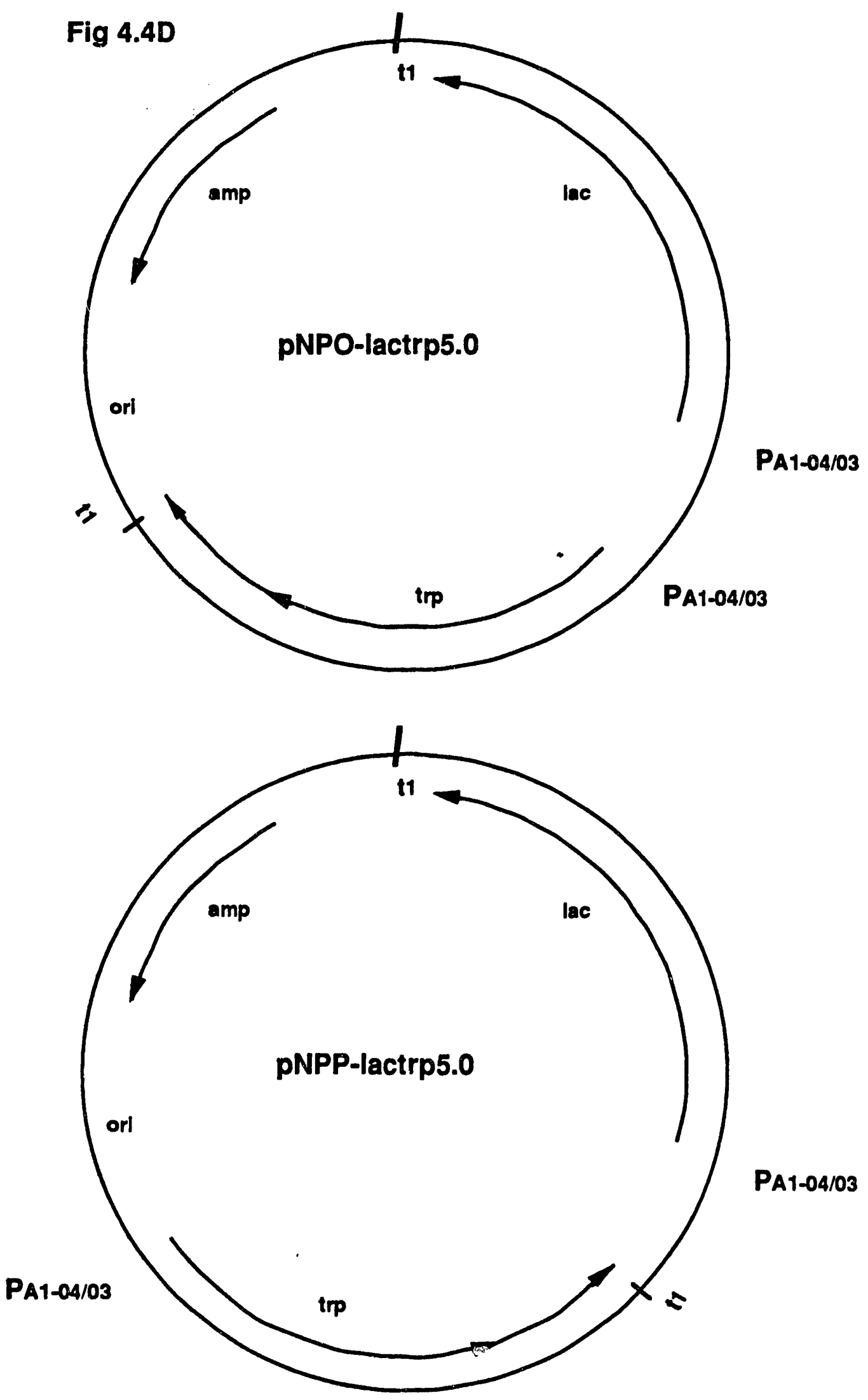
initiates from their own Shine-Dalgarno sequences. The phenotype conferred by this fragment is $\operatorname{trp} A+B+C+D$ - since only a portion of the $\operatorname{trp} D$ gene is included. To make the lac constructs, the $6.2 \mathrm{~kb}$ HindIII-NcoI fragment from pSKS105 was ligated into HindIII-NcoI cut pUHE21.2, resulting in the plasmid pPA1.lac with an in-frame fusion of lacZ to the RBSII and ATG of pUHE21.2. The 304 base pairs from Ncol to XbaI are identical to those in pPA1.trp5.0.

Plasmid pBR322 was next modified to facilitate construction of parallel and opposed constructs. A double-stranded oligomer, containing Xhol, ApaI, and XbaI restriction sites and a 5'-TA overhang at both ends, was chemically synthesized and inserted into the NdeI site of pBR322 (Fig 4.4B). Since the 5'-TA overhang is complementary to NdeI sticky ends, both orientations were generated ("O" = opposed; "P" = parallel). The cloned products were isolated and double-strand sequenced to retain both orientations, yielding the plasmids $\mathrm{pBRXX}-\mathrm{O}$ and $\mathrm{pBRXX}-\mathrm{P}$.

Next, the XhoI-XbaI fragment of pPA1-lac, containing the lac operon, was cloned into both pBRXX-O and pBRXX-P (Fig 4.4C). Cutting pBRXX molecules with Sall and NheI generates overhangs which are compatible with $\mathrm{XhoI}$ and $\mathrm{XbaI}$, respectively. Upon ligation, all of the restriction sites are destroyed. The resulting molecules, pNPO-lac and pNPP.lac, have unique XhoI and Xbal sites formed previously by insertion of the double-stranded oligomer into pBR322.

Finally, the Xhol-Xbal fragment of pPA1-trp5.0 was cloned into both pNPO-lac and pNPP.lac, yielding the desired constructs, pNPO-lactrp5.0 and pNPP.lactrp5.0 (Fig 4.4D). These plasmids differ 
only by the orientation of the trp operon. pNPP-lactrp5.0 should serve as a useful control in testing the twin domain model of transcription.

Construction of a strain permeable to hydrophobic solutes. Most $E$. coli K-12 strains have an outer membrane which excludes small hydrophobic molecules, while being permeable to polar solutes (Nikaido and Vaara, 1985). This unusual property is the consequence of an extensive lipopolysaccharide network (LPS) which coordinates with divalent cations in the growth medium to shield entry of hydrophobic solutes which normally pass through lipid bilayers with ease. Small polar molecules can diffuse through outer membrane porins which form hydrophilic pores, while nonpolar solutes are excluded. D21e7, the permeable mutant of $E$. coli K-12 used in these experiments, was initially isolated as a strain resistant to infection by phage $\mathrm{W}$, which requires an intact LPS in order to enter cells (Monner et al., 1971). Analysis has shown that the $r f a-1$ mutation in D21e7 gives rise to a truncation of the LPS and results in an outer membrane which is highly permeable (Fig 4.5). Both psoralen and novobiocin require a permeable outer membrane in order to effectively enter the cytosol. Although there are other mutants which have additional deletions in the LPS structure, D21e7 is particularly useful since it retains the receptor for phage P1, a common tool in the construction of bacterial strains by transduction (Sandoulachi et al., 1984). Mutants which lack additional portions of the LPS are not sensitive to P1 infection. 
Fig 4.5

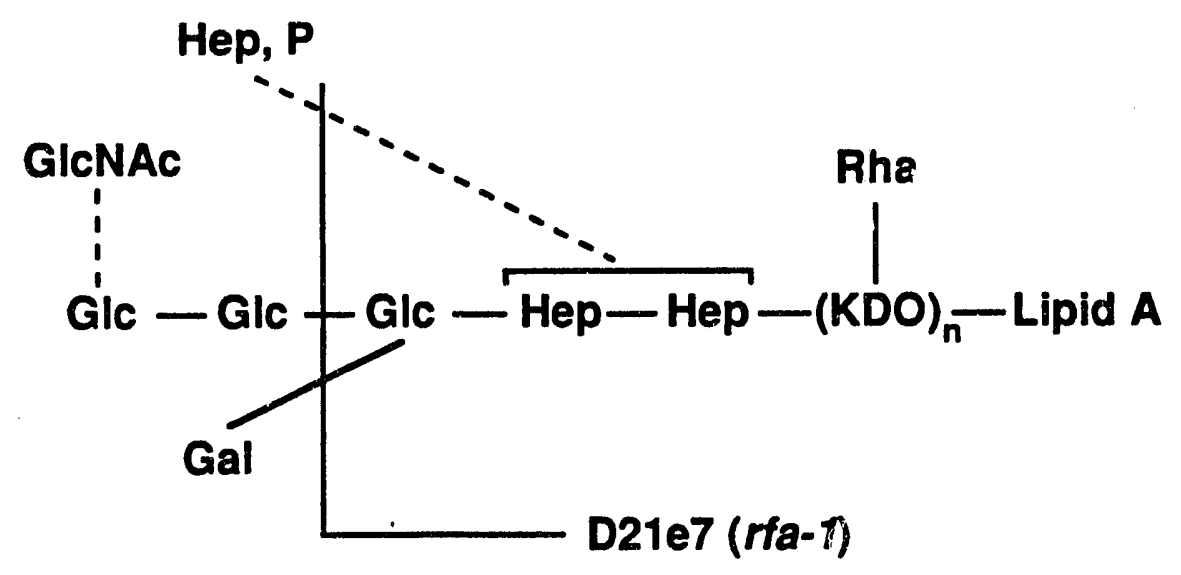

Fig 4.5. The structure of LPS from $E$. coli $\mathrm{K}-12$ and the $r f a-l$ mutant, D21e7. The Lipid A moiety is a complex lipid containing 5 to 6 fatty acid chains attached to a phosphorylated di-glucosamine. $\mathrm{KDO}$ is an abbreviation for 2-keto-3-deoxyoctonic acid, where $\mathrm{n}$ is 2 or 3. Broken lines indicate incomplete substitution. The rfa-a mutation in D21e7 results in the loss of all residues to the left of the solid line. Note that the remaining glucose residue is the essential receptor for phage $\mathrm{P} 1$. Other abbreviations: GlcNAc, N-acetyl-Dgluosamine; Glc, D-glucose; Gal, D-galactose; Hep, L-glycero-Dmanno-heptose; Rha, L-rhamnose. Figure taken from Nikaido and Vaara, 1985. 
The permeability of $\mathrm{D} 21 \mathrm{e} 7$ to novobiocin was demonstrated by the formation of positively supercoiled pBR322 upon treatment with the antibiotic for $30 \mathrm{~min}$. Under the conditions described in Chapter 2 , production of positive supercoils in D21e7 was comparable in efficiency to strain AS19, an E. coli B derivative (data not shown). D21e7 was modified by introduction of a $\operatorname{rec} A$ deletion mutation as described in Materials and Methods. This mutation was introduced in order to insure the stability of the plasmid constructs.

D21, the parent strain of D21e7, was initially isolated for studies of peniciilin resistance and so both strains harbor a chromosomally encoded resistance gene, $a m p C p l$ (Boman et al., 1974). This gene confers complete resistance to ampicillin at a level of $25 \mu \mathrm{g} / \mathrm{ml}$. A titration with ampicillin on plates and in liquid culture indicates that at $200 \mu \mathrm{g} / \mathrm{ml}, \mathrm{D} 21 \mathrm{e} 7$ is not viable (data not shown). Routinely, I selected for the constructs in liquid media and on plates containing $300 \mu \mathrm{g} / \mathrm{ml}$ ampicillin. Under these conditions, plasmids are quite stable, as demonstrated by repeated streaking and subsequent plasmid isolations.

Transcriptional supercoiling in the constructs. In an initial effort to characterize the opposed and parallel constructs, plasmid DNA was isolated from mid-log phase cells before anc after addition of the transcriptional inducer, IPTG, and was then banded in a $\mathrm{CsCl}-\mathrm{EtBr}$ gradient. Control cultures were treated with novobiocin alone or with novobiocin and IPTG (Fig 4.6). In a CsCl-EtBr equilibrium density gradient, topoisomers band at different density based on the amount of ethidium which is intercalated. Negatively supercoiled 
Fig 4.6. CsCl-EtBr density gradients of opposed (top row) and parallel (bottom row) constructs. Plasmid DNA was isolated from cultures treated, left to right, with no inducer or drug, $19 \mathrm{~min}$ IPTG, $15 \mathrm{~min} 80 \mu \mathrm{g} / \mathrm{ml}$ novobiocin, or $4 \mathrm{~min}$ IPTG followed by $15 \mathrm{~min}$ novobiocin. A second band at low density arises only in samples treated with IPTG and novobiocin. 
Fig 4.6. CsCl-EtBr gradients of Opposed and Parallel Constructs

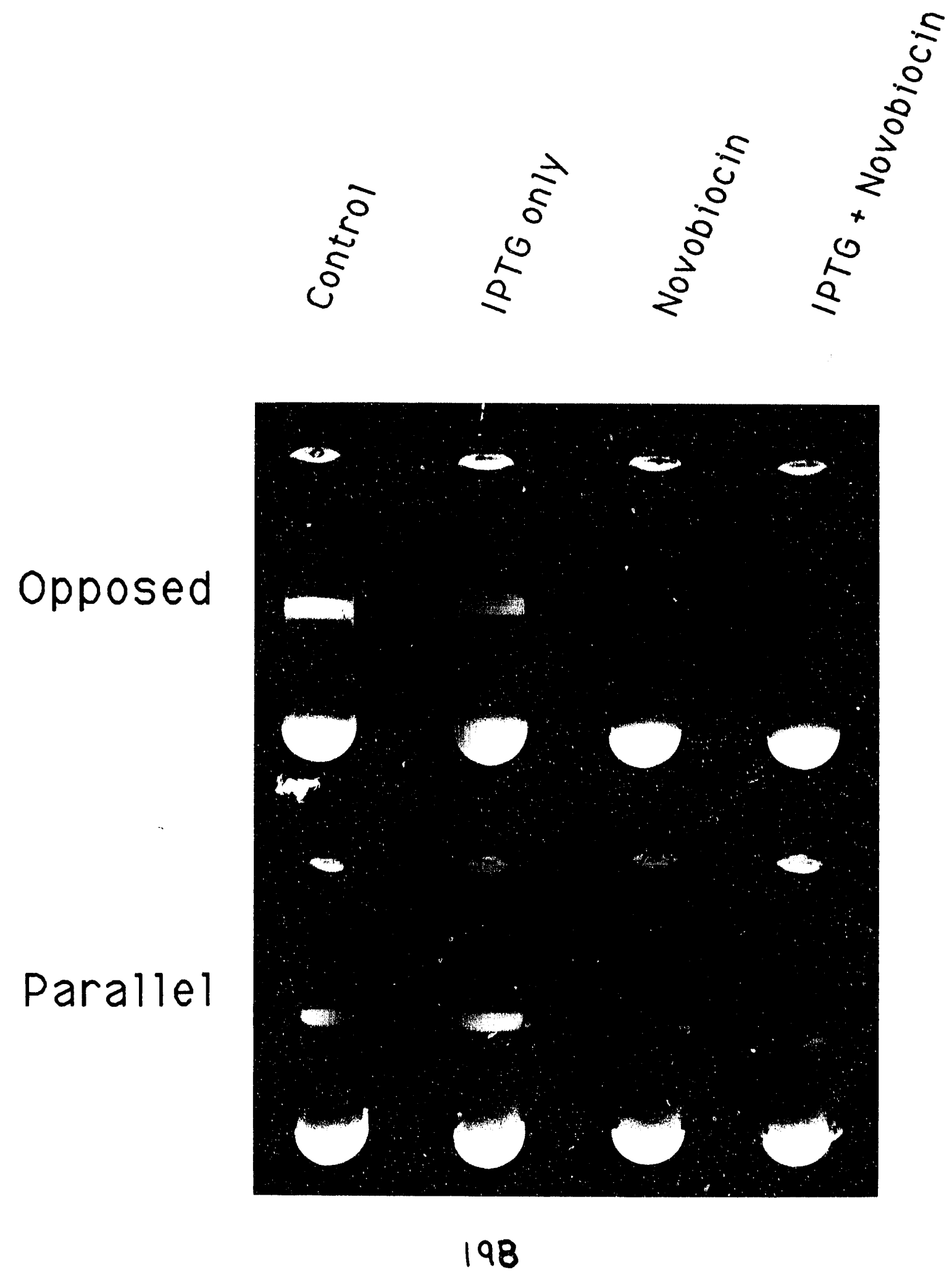


molecules bind more ethidium than relaxed or overwound topoisomers, diminishing their buoyant density. Linear and nicked molecules, which have no topological constrant on intercalation, bind about one ethidium per two base pairs and thus band at the lowest density (Bauer and Vinograd, 1968). In the presence of excess ethidium, as in the gradients shown here, the nicked sample bands at the same absolute density from gradient to gradient. Thus, nicked DNA can serve as a reference from which to measure changes in position of supercoiled DNA.

The striking result in Fig 4.6 is that, for both parallel and opposed constructs, a new band at high density appears in samples treated with novobiocin and IPTG. The position of this band is consistent with the formation of positive supercoils when gyrase is inhibited. In a number of repeat experiments (data not shown), the formation of this species always depends on transcription. It is not formed upon treatment with novobiocin alone. The formation of overwound DNA, independent of gene orientation, is not expected based on the twin domain model alone.

To demonstrate that the high density band is overwound DNA, the following experiment was performed. Plasmid DNA from $400 \mathrm{ml}$ cultures was isolated after addition of IPTG alone, of novobiocin and IPTG, or from control cultures. Each sample was resuspended in a buffer appropriate for topoisomerase I relaxation and split into two fractions. One aliquot of each sample was incubated in the presence of excess topoisomerase $I$. Both aliquots were again loaded onto gradients and spun to equilibrium. The results of this experiment, shown in Fig 4.7, demonstrate that the newly formed band is indeed 
Fig 4.7. $\mathrm{CsCl}-\mathrm{EtBr}$ density gradients of parallel construct from cultures treated with IPTG only (top row) or IPTG and novobiocin (bottom row). Plasmid DNA was isolated from an initial gradient, and haif of the sample was treated with calf thymus topoisomerase I which relaxes both positive and negative supercoils. Nicked DNA was added back to each sample to serve as an internal reference (top band in each tube). The two bands in the IPTG and novobiocin sample are converted to a single band upon treatement with topoisomerase I, indicating that they are topoisomers, with the lower band being overwound relative to the upper. 
Fig 4.7. CsCl-EtBr gradients of Parallel plasmid $+/$ - topoisomerase treatment

$$
(-) \text { topo (+) topo }
$$

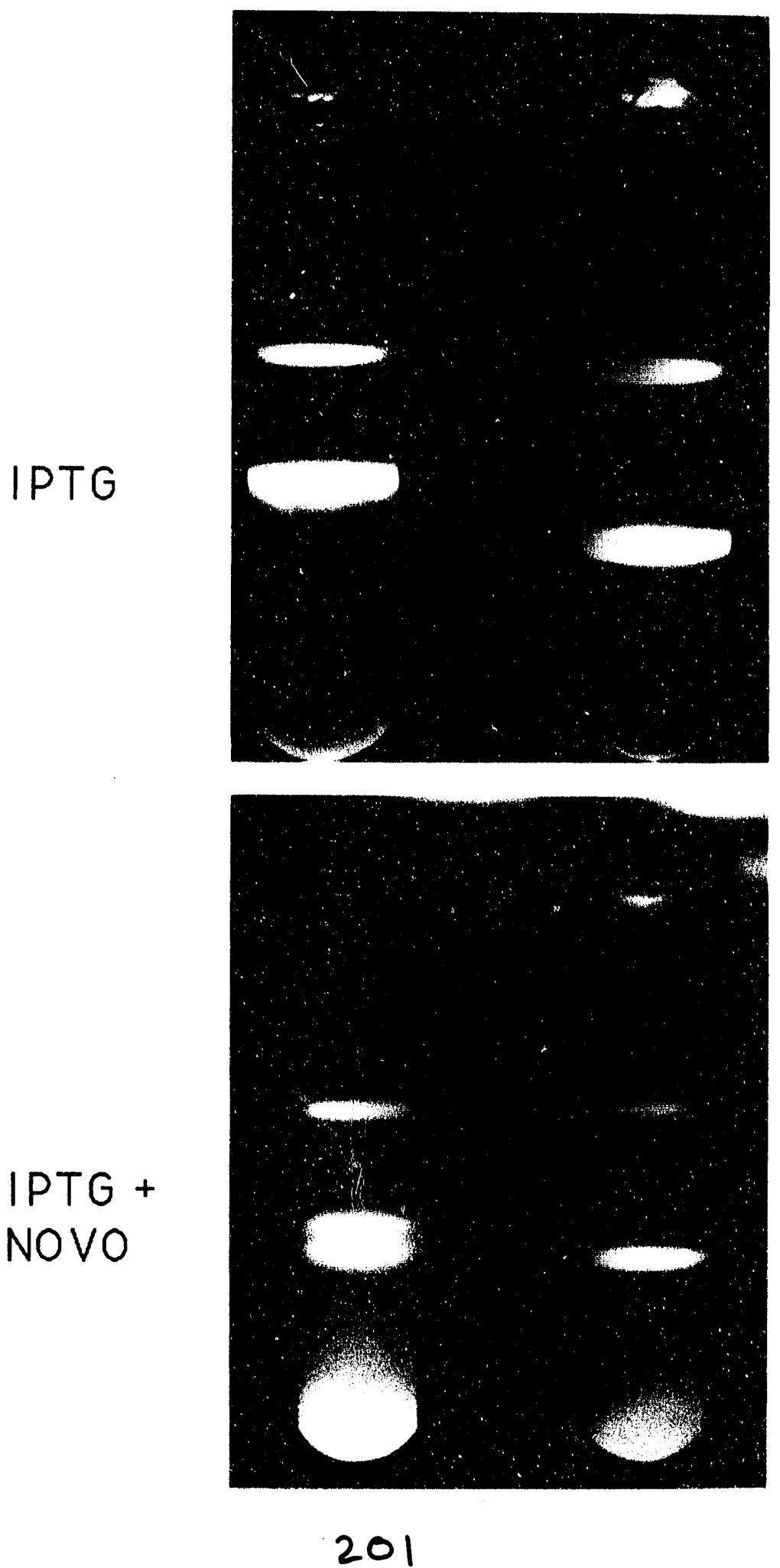


overwound compared to the plasmid before treatment with novobiocin and IPTG. Upon treatment with topoisomerase I, all of the DNA samples band at lower density, showing that they are negatively supercoiled. The high density species, however, bands at about the same position as the relaxed DNA. I conclude, therefore, that the new species is overwound DNA formed by transcription in the absence of DNA gyrase, which traps positive supercoils on the template independently of gene orientation.

Induction of gene expression in the constructs. The induction of lacZ expression for both constructs was measured after addition of IPTG or of novobiocin and IPTG to a log-phase culture. These experiments confirm the conclusion from the $\mathrm{CsCl}-\mathrm{EtBr}$ gradients that opposed and parallel construct respond identically (Fig 4.8). Cultures were induced with IPTG at time zero, and after $4 \mathrm{~min}$, novobiocin was added to half of each culture. The kinetics of accumulation of the LacZ protein are identical in control samples and novobiocin-treated samples for the first $4 \mathrm{~min}$ after addition of the drug. By $8 \mathrm{~min}$ after novobiocin treatment, there is some decrease in LacZ protein compared to the control, and by $12 \mathrm{~min}$, the inhibition of expression by novobiocin is complete. 
Fig 4.8A. Expression of Parallel vs Opposed

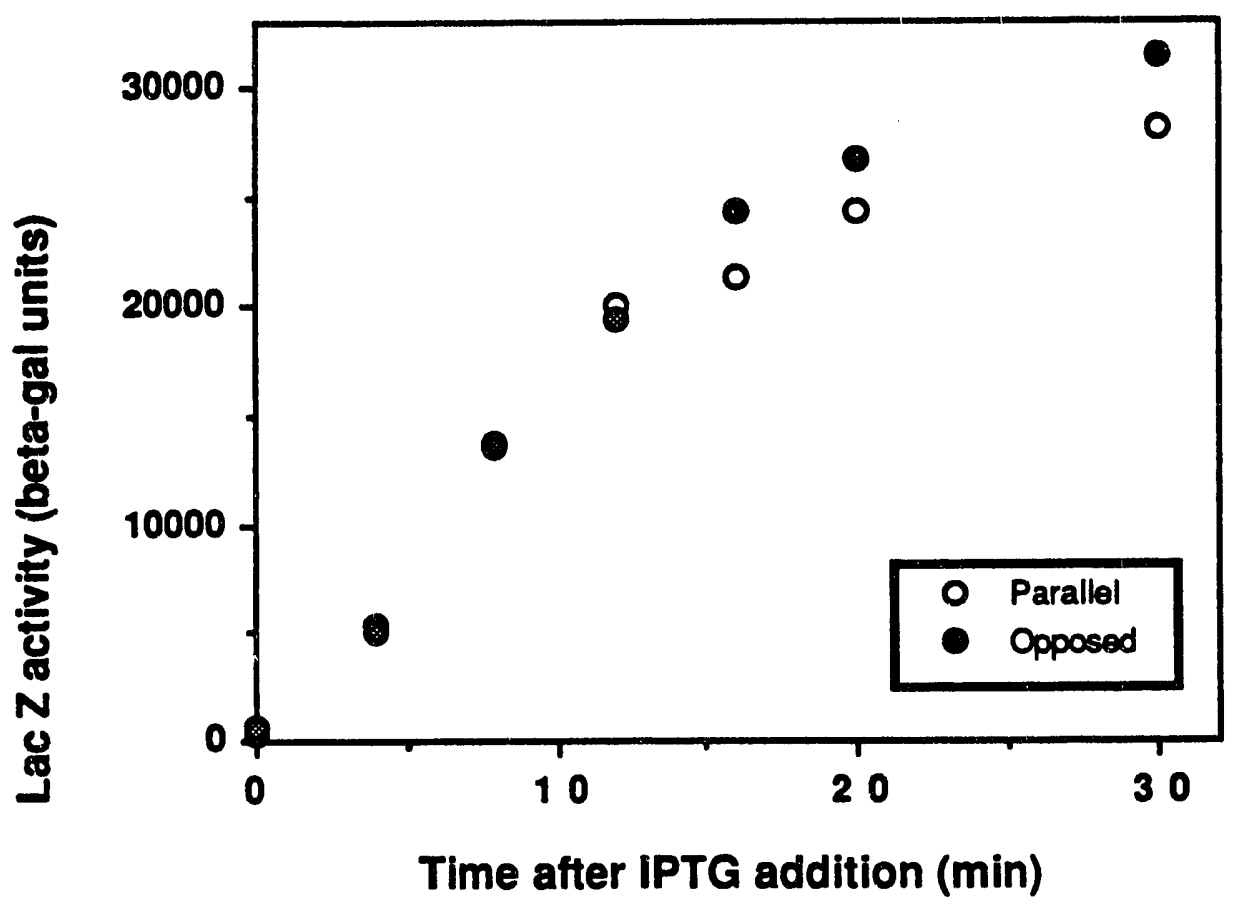

Fig 4.8B. Expression after Novoblocin Treatment

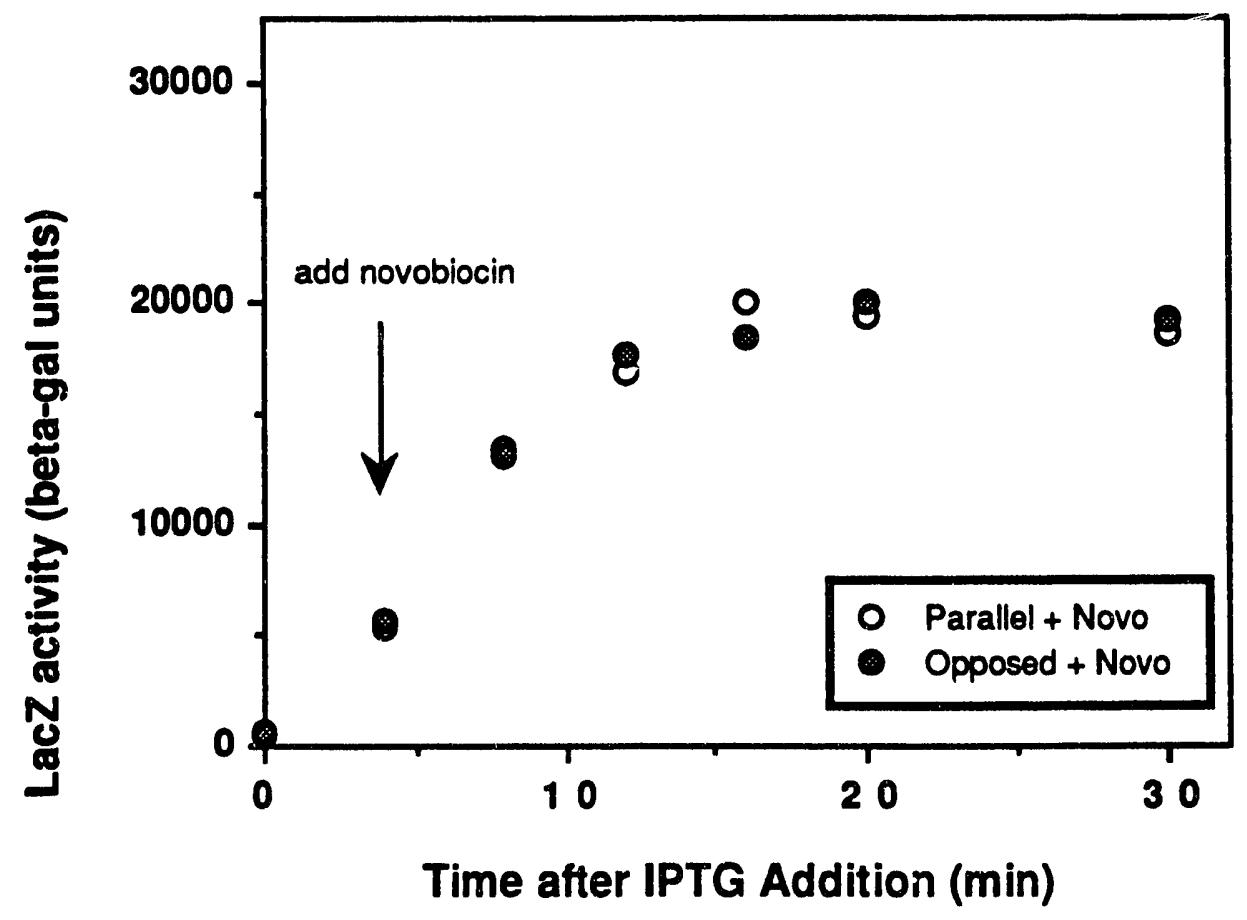




\section{Discussion and Future Work}

I have described the construction of plasmids and a bacterial strain for exploring the mechanisms and consequences of transcriptional supercoiling. The initial results demonstrate that, upon induction of transcription and inhibition of gyrase, overwound DNA is formed independently of gene orientation. The lacZ assay also demonstrates that there is no differential effect of gene orientation on in vivo expression for the constructs described here.

It is significant that overwound DNA species are produced independently of the orientation of transcription. This finding suggests that some other cellular structure--possibly the cell membrane or an unidentified nucleo-protein complex--is anchoring the plasmid DNA so that it has topologically distinct domains. This finding may have general significance since most DNA in cells is much larger than a typical plasmid, and there are facile diffusional pathways for relaxation of transcriptional supercoils on such large molecules. The fact that supercoils are trapped even on the parallel construct implies that some factor other than neighboring opposed transcription units may contribute to the stabilization of transcriptional supercoils.

Future experiments will focus on the following goals:

(1) To determine the mechanism by which these high density bands are formed. The twin domain model leads to the prediction that supercoils generated by transcription of genes in parallel should not contribute to a net change in plasmid superhelicity because the supercoils so formed would annihilate one another. The results presented here imply that supercoils generated by 
transcription on a plasmid may be trapped by an as yet unknown topological anchor. This phenomena was also noted by $\mathrm{Wu}$ et al. (1988) who state in their discussion that formation of positive supercoils on pBR322 occurs even when the promoter for the amp gene is deleted. To determine the topological boundaries on the parallel and opposed plasmids, the psoralen assay described in Chapıer 3 should be particularly useful since it is sensitive to localized changes in superhelix density. It will also be of interest to determine whether transiently overwound DNA can be detected without inhibiting DNA gyrase.

(2) To establish an in vitro transcription system for the study of transcriptional supercoiling. The absence of topoisomerases and other cellular factors in vitro may considerably simplify the interpretation of these experiments. Transcription assays which monitor incorporation of $[\alpha-32 \mathrm{P}] \mathrm{CTP}$ into acid-insoluble material will be used to identify possible effects of supercoiling on the rate of elongation. Formation of topological domains will be investigated using psoralen photochemistry on these transcription reactions. The in vitro experiments provide an opportunity to systematically and selectively vary certain important parameters to investigate the mechanism of transcriptional supercoiling. Variables to be considered in vitro are solvent viscosity, RNA transcript length, and polymerase concentration. Solvent viscosity can be altered by performing transcription reactions in solutions of Ficoll, a non-ionic, synthetic polymer of sucrose. Transcription can take place in concentrated solutions of Ficoll with viscosities approaching 600 times that of water (Cook and Hearst, data not shown). These high 
viscosities approximate those found intracellularly and may dramatically enhance the efficiency of transcriptional supercoiling. RNA transcript length can be varied by replacing the $P_{A 1-04 / 03-}$ trp5.0 fusion with smaller lengths of the trp operon. At some limiting RNA size, the transcription ensemble should generate insufficient torque to supercoil the template. Finally, by varying polymerase concentration, the effects of multiple transcription ensembles on each operon can be investigated. Understanding this parameter in vitro should provide insight to the importance of promoter strength in vivo. Because the available pool of free RNA polymerase does not vary significantly in the cell, it is the strength of a given promoter which determines the number of transcriptions ensembles associated with a gene.

(3) To test the generality of transcriptional supercoiling on the chromosome. The results from the model system should establish the conditions for efficient generation of supercoils by transcription. It is significant that overwound DNA species are produced independently of gene orientation in the constructs. On a large DNA molecule such as the bacterial chromosome, supercoils may be generated by single, strongly expressed transcription units, without requiring a particular orientation of neighboring genes. If this proves to be the case, then transcriptional supercoils may be a fairly general phenomenon. 


\section{References}

Adams, C. W., M. E. Forrest, S. N. Cohen and J. T. Beatty. (1989). Structural and functional analysis of transcriptional control of the Rhodobacter capsulatus puf operon. J. Bacteriol. 171: 473-482.

Adhya, S. (1989). Multipartite genetic control elements: communication by DNA loop. Annu. Rev. Genet. 23: 227-250.

Aggarwal, A. K., D. W. Rodgers, M. Drottar, M. Ptashne and S. C. Harrison. (1988). Recognition of a DNA operator by the repressor of phage 434: a view at high resolution. Science. 242: 899-907.

Allison, S. A. and J. M. Schurr. (1979). Torsion dynamics and depolarization of fluorescence of linear macromolecules. I. Theory and application to DNA. Chem. Phys. 41: 35-59.

Anderson, P. and W. R. Bauer. (1978). Supercoiling in closed circular DNA: dependence upon ion type and concentration. Biochem. 72: 4280 .

Baker, T. A., K. Sekimizu, B. E. Funnell and A. Kornberg. (1986). Extensive unwinding of the plasmid template during staged enzymatic initiation of DNA replication from the origin of the Escherichia coli chromosome. Cell. 45: 53-64. 
Barkley, M. D. and B. Zimm H. (1979). Theory of twisting and bending of chain macromolecules; analysis of the fluorescence deploarization of DNA. J. Chem. Phys. 70: 2991-3007.

Bauer, C. E., D. A. Young and B. L. Marrs. (1988). Analysis of the Rhodobacter capsulatus puf operon. J. Biol. Chem. 263: 4820-4827.

Bauer, W. R., E. C. Ressner, J. Kates, J. V. Patzke and 1977. (1977). A DNA nicking-closing enzyme encapsidated in vaccinia virus: partial purification and proferties. Proc. Natl. Acad. Sci. USA. 74: 18411845 .

Bauer, W. R. and J. Vinograd. (1968). The interaction of closed circular DNA with intercalative dyes. I. The superhelix density of SV40 DNA in the presence and absence of dye. J. Mol. Biol. 33: 141171 .

Bauer, W. R. and J. Vinograd. (1970). Interaction of closed circular DNA with intercalative dyes. II. The free energy of superhelix formation in SV40 DNA. J. Mol. Biol. 47: 419-435.

Beckwith, J. (1987). The lactose operon. Escherichia coli and Salmonella typhimurium: Cellular and molecular biology. Washington, D.C., American Society for Microbiology.

Bhriain, N. N., C. J. Dorman and C. F. Higgins. (1989). An overlap between osmotic and anaerobic stress responses: a potential role for 
DNA supercoiling in the coordinate regulation of gene expression. Mol. Microbiol. 3: 933-942.

Boles, T. C., J. H. White and N. R. Cozzarelli. (1990). Structure of plectonemically supercoiled DNA. J. Mol. Biol. 213: 931-951.

Boman, H. G., K. Nordström and S. Normark. (1974). Penicillin resistance in Escherichia coli $\mathrm{K}-12$ : synergism between penicillinases and a barrier in the outer part of the envelope. Ann. N.Y. Acad. Sci. 235: $569-585$.

Borowiec, J. A. and J. D. Gralla. (1987). All three elements of the lac ps promoter mediate its transcriptional response to DNA supercoiling. J. Mol. Biol. 195: 89-97.

Bramhill, D. and A. Kornberg. (1988). Duplex opening by dnaA protein at novel sequences in initiation of replication at the origin of the E. coli chromosome. Cell. 52: 743-755.

Breslauer, K. J., R. Frank, H. Blocker and L. A. Marky. (1986).

Prediciting DNA duplex stability from the base sequence. Proc. Natl. Acad. Sci. USA. 83: 3746-3750.

Bresloff, J. L. and D. M. Crothers. (1975). DNA-ethidium reaction kinetics: demonstration of direct ligand transfer between DNA binding sites. J. Mol. Biol. 95: 103-123. 
Brewer, B. J. (1988). When polymerases collide: replication and the transcriptional organization of the E. coli chromosome. Cell. 53: 679686.

Brill, S. J. and R. Sternglanz. (1988). Transcription-dependent DNA supercoiling in yeast DNA topoisomerase mutants. Cell. 54: 403411.

Broyles, S. S. and D. E. Pettijohn. (1986). Interaction of the Escherichia coli HU protein with DNA: Evidence for formation of nucleosome-like structures with altered DNA helical pitch. J. Mol. Biol. 187: 47-60.

Büchel, D. E., B. Gronenborn and B. Müller-Hill. (1980). Sequence of the lactose permease gene. Nature. 283: 541-545.

Cairns, J. (1963). The chromosome of E. coli. Cold Spring Harbor Symp. Quant. Biol. 28: 43-46.

Cantor, C. R. and P. R. Schimmel. (1980). Biophysical Chemistry. San Francisco, W. H. Freeman.

Champoux, J. J. and R. Dulbecco. (1972). An activity from mamaiian cells that untwists superhelical DNAs: a possible swivel for DNA replication. Proc. Natl. Acad. Sci. USA. 69: 143-146. 
Chase, J. W. and K. R. Williams. (1986). Single-stranded DNA binding proteins required for DNA replication. Annu. Rev. Biochem. 55: 103-136.

Christman, M. F., F. S. Dietrich and G. R. Fink. (1988). Mitotic recombination in the rDNA of $\mathrm{S}$. cerevisiae is suppressed by the combined action of DNA topoisomerases I and II. Cell. 55: 413-425.

Crick, F. H. C. (1976). Linking numbers and nucleosomes. Proc. Natl. Acad. Sci., USA. 73: 2639-2643.

Csonka, L. N. and A. J. Clark. (1979). Deletions generated by the transposon $\mathrm{Tn} 10$ in the $\operatorname{srl}$ recA region of the Escherichi coli $\mathrm{K}-12$ chromosome. Genetics. 33: 321-343.

Daldal, F., E. Davidson and S. Cheng. (1987). Isolation of the strutural genes for the Rieske Fe-S protein, cytochrome $b$ and cytochrome $c_{1}$, all components of the ubiquinol:cytochrome $c_{2}$ oxidoreductase complex of Rhodopseudomonas capsulata. J. Mol. Biol. 195: 1-12.

De Bernardin, W., T. Koller and J. M. Sogo. (1986). Structure of in vivo transcribing chromatin as studied in Simian Virus 40 minichromosomes. J. Mol. Biol. 191: 469-482.

Depew, R. E. and J. C. Wang. (1975). Conformational fluctuations of the DNA helix. Proc. Natl. Acad. Sci. USA. 72: 4275-4279. 
Deuschle, U., W. Kammerer, R. Gentz and H. Bujar d. (1986). Promoter of Escherichia coli: a hierarchy of in vivo strength indicates alternate structures. EMBO J. 5: 2987-2994.

Dimri, G. P. and H. K. Das. (1988). Transcriptional regulation of nitrogen fixation genes by DNA supercoiling. Mol. Gen. Genet. 212: $360-363$.

DiNardo, S., K. Voekel and R. Sternglanz. (1984). DNA topoisomerase II mutant of Saccharomyces cerevisiae: topoisomerase II is required for segregation of daughter molecules at the termination of DNA replication. Proc. Natl. Acad. Sci. USA. 81: 2616-2620.

DiNardo, S., K. A. Voelkel, R. Sternglanz, A. E. Reynolds and A. Wright. (1982). Escherichia coli DNA topoisomerase I mutants have compensatory mutations in DNA gyrase genes. Cell. 31: 43-51.

Dixon, N. E. and A. Kornberg. (1984). Protein HU in the enzymatic replication of the chromosomal origin of Escherichia coli. Proc. Natl. Acad. Sci., USA. 81: 424-428.

Dixon, R. A., N. C. Henderson and S. Austin. (1988). DNA supercoiling and aerobic regulation of transcription from the Klebsiella pneumoniae nifLA promoter. Nucl. Acids Res. 16: 9933-9946. 
Dorman, C. J., G. C. Barr, N. N. Bhriain and C. F. Higgins. (1988). DNA supercoiling and the anaerobic and growth phase regulation of tonB gene expression. J. Bacteriol. 170: 2816-2826.

Dorman, C. J., N. N. Bhriain and C. F. Higgins. (1990). DNA supercoiling and environmental regulation of virulence gene expression in Shigella flexneri. Nature. 344: 789-792.

Dorman, C. J., A. S. Lynch, N. N. Bhriain and C. F. Higgins. (1989). DNA supercoiling in Escherichia coli: topA mutations can be suppressed by DNA amplifications involving the tolC locus. Mol. Microbiol. 3: $531-540$.

Drew, H. R. and C. R. Calladine. (1987). Sequence-specific positioning of core histones on an 860 base-pair DNA. Experiment and theory. J. Mol. Biol.' 195: 143-173.

Drlica, K. (1984). Biology of bacterial deoxyribonucleic acid topoisomerases. Microbiol. Rev. 48: 273-289.

Drlica, K., E. C. Engle and S. H. Manes. (1980). DNA gyrase action on the bacterial chromosome: possibility of two levels of action. Proc. Natl. Acad. Sci. USA. 77: 6879-6883.

Drlica, K. and M. Snyder. (1978). Superhelical Escherichia coli DNA: relaxation by coumermycin. J. Mol. Biol. 120: 145-154. 
Dunn, T., S. Hahn, S. Ogden and R. Schleif. (1984). An operator at 280 base pairs that is required for repression of $\operatorname{araBAD}$ operon promoter: addition of DNA helical turns between the operator and promoter cyclically hinders repression. Proc. Natl. Acad. Sci. USA. 81: $5017-5020$.

Echols, H. (1986). Multiple DNA-protein interactions governing highprecision DNA transactions. Science. 233: 1050-1056.

Flashner, Y. and J. D. Gralla. (1988). DNA dynamic flexibility and protein recognition: differential stimulation by bacterial histonelike protein HU. Cell. 54: 713-721.

Fleischmann, G., G. Pflugfelder, E. K. Steiner, K. Javaherian, G. C. Howard, J. C. Wang and S. C. R. Elgin. (1984). Drosophila DNA topoisomerase $I$ is associated with transcriptionally active regions of the genome. Proc. Natl. Acad. Sci. USA. 81:

Franco, R. J. and K. Drlica. (1988). DNA gyrase on the bacterial chromosome. Oxolinic acid-induced DNA cleavage in the dnaA-gyrB region. J. Mol. Biol. 201; 229-233.

Frank-Kamenetskii, M. D., A. V. Lukashin, V. V. Anshelvich and A. V. Vologodskii. (1985). Torsional and bending rigidity of the double helix from data on small DNA rings. J. Biomol. Struct. Dyn. 2: 1005 1012. 
Freund, A.-M., M. Bichara and R. P. P. Fuchs. (1989). Z-DNA-forming sequences are spontaneous deletion hot spots. Proc. Natl. Acad. Sci. USA. 86: 7465-7469.

Fujimoto, B. S. and J. M. Schurr. (1990). Dependence of the torsional rigidity of DNA on base composition. Nature. 344: 175-178.

Fuller, F. B. (1971). The writhing number of a space curve. Proc. Natl. Acad. Sci. USA. 68: 815-819.

Gamper, H. B. and J. E. Hearst. (1982). A topological model for transcription based on unwinding angle analysis of $E$. coli RNA polymerase binary, initiation, and ternary complexes. Cell. 29: 81 90.

Gartenberg, M. R. and D. M. Crothers. (1988). DNA sequence determinants of CAP-induced bending and protein binding affinity. Nature. 333: 824-829.

Gellert, M. (1981). DNA topoisomerases. Ann. Rev. Biochem. 50: 879-910.

Gellert, M., K. Mizuuchi, M. H. O'Dea, T. Itoh and J.-I. Tomizawa. (1977). Nalidixic acid resistance: a second genetic character involved in DNA gyrase activity. Proc. Natl. Acad. Sci., USA. 74: $4772-4776$. 
Gellert, M., K. Mizuuchi, M. H. O'Dea and H. A. Nash. (1976). DNA gyrase: an enzyme that introduces superhelical turns into DNA. Proc. Natl. Acad. Sci. USA. 73: 3872-3876.

Gellert, M., M. H. O'Dea, T. Itoh and J.-I. Tomizawa. (1976).

Novobiocin and coumermycin inhibit DNA supercoiling catalyzed by DNA gyrase. Proc. Natl. Acad. Sci. USA. 73: 4474-4478.

Giaever, G. N. and J. C. Wang. (1988). Supercoiling of intracellular DNA can occur in eukaryotic cells. Cell. 55: 849-856.

Gilmour, D. S., G. Pflugfelder, J. C. Wang and J. T. Lis. (1986).

Topoisomerase $I$ interacts with transcribed regions in Drosophila cells. Cell. 44: 401-407.

Glaubiger, D. and J. E. Hearst. (1967). Effect of superhelical structure of DNA rings. Biopolymers. 5: 691-696.

Goldstein and K. Drlica. (1984). Regulation of bacterial supercoiling: plasmid linking numbers vary vith growth temperature. Proc. Natl. Acad. Sci. USA. 81: 4046-4050.

Goto, T. and J. C. Wang. (1985). Cloning of yeast TOP1, the gene encoding DNA topoisomerase $I$, and construction of mutants defective in both DNA topoisomerase I and DNA topoisomerase II. Proc. Natl. Acad. Sci. USA. 82: 7178-7182. 
Graeme-Cook, K. A., G. May, E. Bremer and C. F. Higgins. (1989).

Osmotic regulation of porin expression: a role for DNA supercoiling. Mol. Microbiol. 3: 1287-1294.

Gray, E. D. (1967). Studies on the adaptive formation of photsynthetic structures in Rhodopseudomonas spheroides I. Synthesis of macromolecules. Biochem. Biophys. Acta. 138: 550563.

Griffith, J., A. Hochschild and M. Ptashne. (1986). DNA loops induced by cooperative binding of lambda repressor. Nature. 322: 750-752.

Griffith, J. D. and H. A. Nash. (1985). Genetic rearrangement of DNA induces knots with a unique topology: implications for the mechanism of synapsis and crossing over. Proc. Natl. Acad. Sci. USA. 82: $3124-3128$.

Hagerman, P. J. and V. A. Ramadevi. (1990). Application of the method of phage T4 DNA ligase-catalyzed ring-closure to the study of DNA structure. I. Computational analysis. J. Mol. Biol. 212: 351 362.

Havekes, L., J. Townasen, W. Hoekstra and B. Lugtenberg. (1977). Isolation and characterization of Escherichia coli $\mathrm{K}-12 \mathrm{~F}^{-}$mutants defective in conjugation with an I-type donor. J. Bacteriol. 129: 1. 
Hediger, M. A., D. F. Johnson and D. P. Nierlich. (1985). DNA sequence of the lactose operon: The lacA gene and the transcriptional termination region. Proc. Natl. Acad. Sci. USA. 82: 6414-6418.

Higgins, C. F., C. J. Dorman, D. A. Stirling, L. Waddell, I. R. Booth, G. May and E. Bremer. (1988). A physiological role for DNA supercoiling in the osmotic regulation of gene expression in $S$. typhimurium and $E$. coli. Cell. 52: 569-584.

Hochschild, A. and M. Ptashne. (1986). Cooperative binding of lambda repressors to sites separated by integral turns of the DNA helix. Cell. 44: 681-687.

Hodges-Garcia, Y., P. Hagerman and D. E. Pettijohn. (1989). DNA ring closure mediated by protein HU. J. Biol. Chem. 264: 14621-14623.

Hogan, M., N. Dattagupta and D. M. Crothers. (1979). Transient electric dichroism studies of the structure of the DNA complex with intercalated drugs. Biochem. 18: 280-288.

Horowitz, D. S. and J. C. Wang. (1984). Torsional rigidity of DNA and length dependence of the free energy of DNA supercoiling. J. Mol. Biol. 173: 75-91. 
Horwitz, M. S. Z. and L. A. Loeb. (1988). An E. coli promoter that regulates transcription by DNA superhelix-induced cruciform extrusion. Science. 241: 703-705.

Htun, H. and J. E. Dahlberg. (1989). Topology and formation of triplestranded H-DNA. Science. 243: 1571-1576.

Hyde, J. E. and J. E. Hearst. (1978). Binding of psoralen derivatives to DNA and chromatin: influence of the ionic environment on dark binding and photoreactivity. Biochemistry. 17: 1251-1257.

Jaworski, A., W.-T. Hsieh, J. A. Blaho, J. E. Larson and R. D. Wells. (1987). Left-handed DNA in vivo. Science. 238: 773-777.

Jayram, B., K. A. Sharp and B. Honig. (1989). The electrostatic potential of B-DNA. Biopolymers. 28: 975-993.

Johnson, R. C., M. F. Bruist and M. I. Simon. (1986). Host protein requirements for in vitro site-specific DNA inversion. Cell. 46: 531539.

Johnston, B. H., M. A. Johnson, C. B. Moore and J. E. Hearst. (1977). Psoralen-DNA photoreaction: controlled production of mono- and diadducts with nanosecond ultraviolet laser pulses. Science. 197: 906-908. 
Kalnins, A., K. Otto, U. Rüther and B. Müller-Hill. (1983). Sequence of the lacZ gene of Escherichia coli. EMBO J. 2: 593-597.

Keller, W. (1975). Determination of the number of superhleical turns in simian virus 40 DNA by gel electrophoresis. Proc. Natl. Acad. Sci., USA. 72: $4876-4880$.

Kikuchi, A. and K. Asai. (1984). Reverse gyrase: a topoisomerase which introduces positive superhelical turns into DNA. Nature. 309: $677-681$.

Kim, R. A. and J. C. Wang. (1989). A subthreshold level of DNA topoisomerases leads to the excision of yeast rDNA as extrachromosomal rings. Cell. 57: 975-985.

Kim, R. and S.-H. Kim. (1983). Direct measurement of DNA unwinding angle in specific interaction between lac operator and repressor. Cold Spring Harbor Sym. Quant. Biol. 47: 451-454.

Kirkegaard, K. and J. C. Wang. (1981). Mapping the topography of DNA wrapped around gyrase by nucleolytic and chemical probing of complexes of unique DNA sequences. Cell. 23: 721-729.

Kirkegaard, K. and J. C. Wang. (1985). Bacterial DNA topoisomerase I can relax positively supercoiled DNA containing a single-stranded loop. J. Mol. Biol. 185: 625-637. 
Klug, A., D. Rhodes, J. Smith, J. T. Finch and J. O. Thomas. (1980). A low resolution structure for the histone core of the nucleosome. Nature. 287: 509-516.

Koo, H.-S., H.-Y. Wu, and L. F. Liu. (1990). Effects of transcription and translation on gyrase-mediated DNA cleavage in Escherichia coli. J. Biol. Chem. 265: 12300-12305.

Koudelka, G. B., P. Harbury, S. C. Harrison and M. Ptashne. (1988). DNA twisting and the affinity of bacteriophage 434 operator for bacteriophage 434 repressor. Proc. Natl. Acad. Sci. USA. 85: 46334637.

Koudelka, G. B., S. C. Harrison and M. Ptashne. (1987). Effect of noncontacted bases on the affinity of 434 operator for 434 repressor and Cro. Nature. 326: 886-888.

Kramer, H., M. Amouyal, A. Nordheim and B. Muller-Hill. (1988). DNA supercoiling changes the spacing requirement of two lac operators for DNA loop formation with lac repressor. EMBO J. 7: 547-556.

Kramer, H., M. Niemoller, M. Amouyal, B. Revet, B. von WilckenBergmann and B. Muller-Hill. (1987). lac repressor forms loops with linear DNA carrying two suitably spaced lac operators. EMBO J. 6: $1481-1491$. 
Kranz, R. G. and R. Haselkorn. (1986). Anaerobic regulation of nitrogen-fixation genes in Rhodopseudomonas capsulata. Proc. Natl. Acad. Sci., USA. 83: 6805-6809.

Kreuzer, K. N. and N. R. Cozzarelli. (1979). Escherichia coli mutants thermosensitive for deoxyribonucleic acid gyrase subunit A. Effects on deoxyribonucleic acid replication, transcription, and bacteriophage growth. J. Bacteriol. 140: 424-435.

Lakowicz, J. R. (1983). Principles of Fluorescence Spectroscopy. New York, Plenum Press.

Lamond, A. I. (1985). Supercoiling response of a bacterial tRNA gene. EMBO J. 4: 501-507.

LePecq, J.-B. and C. Paoletti. (1967). A fluorescent complex between ethidium bromide and nucleic acids. J. Mol. Biol. 27: 87-106.

Levene, S. D. and D. M. Crothers. (1986). Topological distributions and the torsional rigidity of DNA: a Monte Carlo study of DNA circles. J. Mol. Biol. 189: 73-83.

Liu, L. F. and C.-C. Liu. (1980). Type II DNA topoisomerases: enzymes that can unknot a topologically knotted DNA molecule via a reversible double-strand break. Cell. 19: 697-707. 
Liu, L. F., C.-C. Liu and B. M. Alberts. (1979). T4 DNA topoisomerase: a new ATP-dependent enqyme essential for initiation of T4 bacteriophage DNA replication. Nature. 281: 456-461.

Liu, L. F. and J. C. Wang. (1978). DNA-DNA gyrase complex: the wrapping of the DNA duplex outside the enzyme. Cell. 15: 979-984.

Liu, L. F. and J. C. Wang. (1987). Supercoiling of the DNA template during transcription. Proc. Natl. Acad. Sci., USA. 84: 7024-7027.

Liu-Johnson, H. N., M. R. Gartenberg and D. M. Crothers. (1986). The DNA binding domain and bending angle of E. coli CAP protein. Cell. 47: $995-1005$.

Lockshon, D. and D. R. Morris. (1983). Positively supercoiled plasmid DNA is produced by treatment of Escherichia coli with DNA gyrase inhibitors. Nucleic Acids Res. 11: 2999-3017.

Lodge, J., T. Kazic and D. E. Berg. (1989). Formation of supercoiling domains in plasmid pBR322. J. Bacteriol. 171: 2181-2187.

Lyamichev, V. I., S. M. Mirkin and M. D. Frank-Kamenetskii. (1985). A pH-dependent structural transition in the homopurinehomopyrimidine tract in superhelical DNA. J. Biomol. Struct. Dyn. 3: 327-338. 
Lynn, R., G. Giaever, S. L. Swanberg and J. C. Wang. (1986). Tandem regions of yeast DNA topoisomerase II share homology with different subunits of bacterial gyrase. Science. 233: 647-649.

Majumdar, A. and S. Adhya. (1984). Demonstration of two operator elements in gal: in vitro repressor binding studies. Proc. Natl. Acad. Sci. USA. 81: 6100-6104.

Manes, S. H., G. J. Pruss and K. Drlica. (1983). Inhibition of RNA synthesis by oxolonic acid is unrelated to average DNA supercoiling. J. Bacteriol. 155: 420-423.

Marquardt, D. W. (1963). An algorithm for least squares estimation of nonlinear parameters. J. Soc. Ind. Appl. Math. 11: 431-441.

Marrs, B. (1981). Mobilization of the genes for photosynthesis from Rhodopseudomonas capsulata by a promiscuous plasmid. J. Bacteriol. 146: $1003-1012$.

Maxwell, A. and M. Gellert. (1986). Mechanistic aspects of DNA topoisomerases. Adv. Prot. Chem. 38: 69-109.

McClure, W. R. (1985). Mechanism and control of transcription initiation in prokaryotes. Annu. Rev. Biochem. 54: 171-204. 
Menzel, R. and M. Gellert. (1983). Regulation of the genes for E. coli DNA gyrase: homeostatic control of DNA supercoiling. Cell. 34: 105-11?.

Menzel, R. and M. Gellert. (1987a). Fusions of the Escherichia coli gyrA and gyrB control regions to the galactokinase gene are inducible by coumermycin treatment. J. Bacteriol. 169: 1272-1278.

Menzel, R. and M. Gellert. (1987b). Modulation of transcription by DNA supercoiting: a deletion analysis of the Escherichia coli gyrA and gyrB promoters. Proc. Natl. Acad. Sci. USA. 84: 4185-4189.

Millar, D. P., R. J. Robbins and A. H. Zewail. (1980). Direct observation of the torsional dynamics of DNA and RNA by picosecond spectroscopy. Proc. Natl. Acad. Sci., USA. 77: 5593-5597.

Millar, D. P., R. J. Robbins and A. H. Zewail. (1982). Torsion and bending of nucleic acids studies by subnanosecond time-resolved fluorescence depolarization of intercalated dyes. J. Chem. Phys. 76: 2080-2094.

Miller, J. H. (1972). Experiments in Molecular Genetics. Cold Spring Harbor, NY, Cold Spring Harbor Laboratories.

Mizuuchi, K., M. Gellert and H. A. Nash. (1978). Involvement of supertwisted DNA in integrative $r$ zombination of bacteriophage lambda. J. Mol. Biol. 212: 375-392. 
Mizuuchi, K., M. Mizuuchi and M. Gellert. (1982). Cruciform structures in palindromic DNA are favored by DNA supercoiling. J. Mol. Biol. 156: 229-243.

Mizuuchi, K. and H. A. Nash. (1976). Restriction assay for integrative recombination of bacteriophnage 1 DNA in vitro: requirement for closed circular DNA substrate. Proc. Natl. Acad. Sci. USA. 73: 3524 3528 .

Monner, D. A., S. Jonsson and H. G. Boman. (1971). Ampicillinresistant mutants of Escherichia coli K-12 with lipopolysaccharide alterations affecting mating ability and susceptibility to sex-specific bacteriophages. J. Bacteriol. 107: 420-432.

Morrison, A. and N. R. Cozzarelli. (1979). Site-specific cleavage of DNA by E. coli DNA gyrase. Cell. 17: 175-184.

Müller, M. T., W. P. Pfund, V. B. Mehta and D. K. Trask. (1985). Eukaryotic type I topoisomerase is enriched in the nucleolus and catalytically active on ribosomal DNA. EMBO J. 4: 1237-1243.

Nash, H., A. (1990). Bending and supercoiling of DNA at the attachment site of bacteriophage $\lambda$. TIBS. 15: 222-227.

Nikaido, H. and M. Vaara. (1985). Molecular basis of bacterial outer membrane permeability. Microbiol. Rev. 49: 1-32. 
Novak, P. D. and R. J. Maier. (1987). Inhibition of hydrogenase synthesis by DNA gyrase inhibitors in Bradyrhizobium japonicum. J. Bacteriol. 169: 2708-2712.

O'Connor, D. and D. Phillips. (1984). Time-correlated Single Photon Counting. London, Academic Press.

Ogden, G. B., M. J. Pratt and M. Schaechter. (1988). The replicative origin of the $E$. coli chromosome binds to cell membranes only when hemimethylated. Cell. 54: 127-135.

Olins, A. L. and E. E. Olins. (1974). Spheroid chromatin units (v bodies). Science. 183: 330-332.

Paulson, J. R. and U. K. Laemmli. (1977). The structure of histonedepleted metaphase chromosomes. Cell. 12: 817-828.

Peck, L. J. and J. C. Wang. (1981). Sequence dependence of the helical repeat of DNA in solution. Nature. 292: 375-378.

Pettijohn, D. E. (1988). Histone-like proteins and bacterial chrornosome structure. J. Biol. Chem. 263: 12793-12796.

Pruss, G. J. and K. Drlica. (1989). DNA supercoiling and prokaryotic transcription. Cell. 56: 521-523. 
Pruss, G. J. and K. Drlica. (1986). Topoisomerase I mutants: the gene of pBR322 that encodes resistance to tetracycline affects plasmid DNA supercoiling. Proc. Natl. Acad. Sci. USA. 83: 8952-8956.

Pruss, G. J., S. H. Manes and K. Drlica. (1982). Escherichia coli DNA topoisomerase I mutants: increased supercoiling is corrected by mutations near gyrase genes. Cell. 31: 35-42.

Pulleyblank, D. E., M. Shure, D. Tang, J. Vinograd and H. P. Vosberg. (1975). Action of nicking-closing enzyme on supercoiled and nonsupercoiled closed circular DNA: formation of a Boltzmann distribution of topological isomers. Proc. Natl. Acad. Sci. USA. 72: $4280-4284$.

Rahmouni, A. R. and R. D. Wells. (1989). Stabilization of Z DNA in vivo by localized supercoiling. Science. 246: 358-363.

Rhodes, D. and A. Klug. (1980). Helical periodicity of DNA determined by enzyme digestion. Nature. 286:

Rich, A., A. Nordhein and A. H.-J. Wang. (1984). The chemistry and biology of left-handed Z DNA. Annu. Rev. Biochem. 53:

Richardson, J. P. (1975). Initiation of transcription by Escherichia coli RNA polymerase from supercoiled and nonsupercoiled bacteriophage PM2 DNA. J. Mol. Biol. 91: 477-487. 
Richardson, S. M., T. C. Boles and N. R. Cozzarelli. (1988). The helical repeat of underwound DNA in solution. Nucleic Acids Res. 16: 6607-6616.

Richardson, S. M. H., C. F. Higgins and D. M. J. Lilley. (1988). DNA supercoiling and the leu-500 promoter mutation of Salmonella typhimurium. EMBO J. 7: 1863-1869.

Richet, E., P. Abcarian and H. A. Nash. (1986). The interaction of recombination proteins with supercoiled DNA: defining the role of supercoiling in lambda integrative recombination. Cell. 46: 1011 1021 .

Robinson, B. H., L. S. Lerman, A. H. Beth, H. L. Frisch, L. R. Dalton and C. Auer. (1980). Analysis of double-helix motions with spin-labeled probes: binding geometry and the limit of torsional plasticity. J. Mol. Biol. 139: 19.

Sambrook, J., E. F. Frisch and T. Maniatis. (1989). Molecular Cloning: A Laboratory Manual. Cold Spring Harbor, Cold Spring Harbor Laboratory Press.

Sandoulachi, R., P. Prehm and D. Damp. (1984). Cell wall receptor for bacteriophage $\mathrm{Mu} \mathrm{G(+).} \mathrm{J.} \mathrm{Bacteriol.} \mathrm{160:} \mathrm{299-303.}$

Sanzey, B. (1979). Modulation of gene expression by drugs affecting deoxyribonucleic acid gyrase. J. Bacteriol. 138: 40-47. 
Scalettar, B. A., P. R. Selvin, D. Axelrod, M. P. Klein and J. E. Hearst. (1990). A polarized photobleaching study of DNA reorientation in agarose gels. Biochem. 29: 4790-4798.

Schneider, W. P., B. P. Nichols and C. Yanofsky. (1981). Procedure for production of hybrid genes and proteins and its use in assessing significance of amino acid differences in homologous tryptophan synthetase $\alpha$ peptides. Proc. Natl. Acad. Sci. USA. 78: 2169-2173.

Schurr, J. M. (1984). Rotational diffusion of deformable macromolecules with mean local cylindrical symmetry. Chem. Phys. 84: $71-96$.

Shibata, J. H., J. Wilcoxon, J. M. Schurr and V. Knauf. (1984). Structures and dynamics of a supercoiled DNA. Biochemistry. 23: $1188-1194$

Shimada, J. and H. Yamakawa. (1984). Ring-closure probabilities for twisted wormlike chains. Applications to DNA. Macromolecules. 17: 689-698.

Shore, D. and R. L. Baldwin. (1983a). Energetics of DNA twisting. I. Relation between twist and cyclization probability. J. Mol. Biol. 170: 957-981. 
Shore, D. and R. L. Baldwin. (1983b). Energetics of DNA twisting. II. Topoisomer analysis. J. Mol. Biol. 170: 983-1007.

Sinden, R. R., S. S. Broyles and D. E. Pettijohn. (1983). Perfect palindromic lac operator DNA sequence exists as a stable cruciform structure in supercoiled DNA in vitro but not in vivo. Proc. Natl. Acad. Sci. USA. 80: 1797-1801.

Sinden, R. R., J. O. Carlson and D. E. Pettijohn. (1980). Torsional tension in the DNA double helix measured with trimethylpsoralen in living $E$. coli cells: analagous measurements in insect and human cells. Cell. 21: 773-783.

Sinden, R. R. and D. E. Pettijohn. (1981). Chromosomes in living Escherichia coli cells are segregated into domains of supercoiling. Proc. Natl. Acad. Sci., USA. 78: 224-228.

Sinden, R. R. and D. E. Pettijohn. (1982). Torsional tension in intracellular bacteriophage T4 DNA. J. Mol. Biol. 162: 659-677.

Singleton, C. K. and R. D. Wells. (1982). The facile generation of covalently closed, circular DNAs with defined negative superhelical densities. Anal. Biochem. 122: 253-257.

Smith, O. H. and C. Yanofsky. (1962). Enzymes involved in the biosynthesis of tryptophan. Methods Enzymol. 5: 794-806. 
Snyder, M. and K. Drlica. (1979). DNA gyrase on the bacterial chromosome: DNA cleavage induced by oxolinic acid. J. Mol. Biol. 131: $287-302$.

Sogo, J. M., H. Stahl, T. Koller and R. Knippers. (1986). Structure of replicating Simian Virus 40 minichromosomes: The replication fork, core histone segregation and terminal structures. J. Mol. Biol. 189: 189-204.

Song, L., B. S. Fujimoto, P. Wu, J. C. Thomas, H. H. Shibata and J. M. Schurr. (1990). Evidence for allosteric transitions in secondary structure induced by superhelical stress. J. Mol. Biol. 214: 307-326.

Steck, T. R. and K. Drlica. (1984). Bacterial chromosome segregation: evidence for DNA gyrase involvement in decatenation. Cell. 36: $1081-1088$.

Steck, T. R., G. J. Pruss, S. H. Manes, L. Burg and K. Drlica. (1984). DNA supercoiling in gyrase mutants. J. Bacteriol. 158: 397-403.

Sternglanz, R., S. DiNardo, K. A. Voelkel, Y. Nishimura, Y. Hiroto, L. Becherer, L. Zumstein and J. C. Wang. (1981). Mutations in the gene coding for Escherichia coli DNA topoisomerase I affecting transcription. Proc. Natl. Acad. Sci. USA. 78: 2747-2751.

Stirling, D. A., C. S. Hulton, L. Waddell, S. F. Park, G. S. Stewart, I. R. Booth and C. F. Higgins. (1989). Molecular characterization of the 
proU loci of Salmonella typhimurium and Escherichia coli encoding osmoregulated glycine betaine transport systems. Mol. Microbiol. 3: 1025-1038.

Sugino, A., N. P. Higgins, P. O. Brown, C. L. Peebles and N. R. Cozzarelli. (1978). Energy coupling in DNA gyrase and the mechanism of action of novobiocin. Proc. Natl. Acad. Sci. USA. 75: 4838-4842.

Sugino, A., C. L. Peebles, K. N. Kreuzer and N. R. Cozzarelli. (1977). Mechanism of action of nalidixic acid: purification of Escherichia coli nalA gene product and its relationship to DNA gyrase and a novel nicking-closing enzyme. Proc. Natl. Acad. Sci. USA. 74: 4767-4771.

Taylor, D. P., S. N. Cohen, W. G. Clark and B. L. Marrs. (1983). Alignment of genetic and restriction maps of the photosynthesis region of Rhodopseudomonas capsulata chromosome by a conjugation-mediated marker rescue technique. J. Bacteriol. 154: 580-590.

Taylor, W. H. and P. J. Hagerman. (1990). Application of the method of phage T4 DNA ligase-catalyzed ring-closure to the study of DNA structure. II. NaCl-dependence of DNA flexibility and helical repeat. J. Mol. Biol. 212: 363-376.

Thomas, J. C., S. A. Allison, C. J. Appellof and J. M. Schurr. (1980). Torsion dynamics and depolarization of fluorescence of linear macromolecules. II. Fluorescence polarization anisotropy 
measurements on a clean viral $\$ 29$ DNA. Biophys. Chem. 12: 177188.

Thomas, J. C. and J. M. Schurr. (1983). Fluorescence depolarization and temperature dependence of the torsion elastic constant of linear Ф29 deoxyribonucleic acid. Biochem. 22: 6194-6198.

Thrash, C., A. T. Bankier, B. G. Barrell and R. Sternglanz. (1985).

Cloning, characterization and sequence of the yeast DNA topoisomerase I gene. Proc. Natl. Acad. Sci. USA. 82: 4374-4378.

Torbet, J. and E. DiCapua. (1989). Supercoiled DNA is interwound in liquid crystalline solutions. EMBO J. 8: 4351-4356.

Tsao, Y.-P., H.-Y. Wu and L. F. Liu. (1989). Transcription-driven supercoiling of DNA: direct biochemical evidence from in vitro studies. Cell. 56: 111-118.

Tse, Y.-C. and J. C. Wang. (1980). E. coli and M. luteus DNA topoisomerase I can catalyze catenation or decatenation of doublestranded rings. Cell. 22: 269-276.

Tse-Dinh, Y.-C. (1985). Regulation of the Escherichia coli DNA topoisomerase I gene by DNA supercoiling. Nucleic Acids Res. 13: 4751-4763. 
Tse-Dinh, Y.-C. and R. K. Beran. (1988). Multiple promoters for transcription of the Escherichia coli DNA topoisomerase I gene and their regulation by DNA supercoiling. J. Mol. Biol. 202: 735-742.

Volishin, O. N., S. M. Mirkin, V. I. Lyamichev, B. P. Belotserkovskii and M. D. Frank-Kamenetskii. (1988). Chemical probing of homopurine-homopyrimidine mirror repeats in supercoiled DNA. Nature. 333: 475-476.

Vologodskii, A. V., V. V. Anshelevish, A. V. Lukashin and M. D. Frank-Kamenetskii. (1979). Statistical mechanics of supercoils and the torsional stiffness of the DNA double helix. Nature. 280: 294298.

Vos, J.-M. H. and P. C. Hannawalt. (1987). Processing of psoralen adducts in an active human gene: repair and replication of DNA containing monoadducts and interstrand cross-links. Cell. 50: 789799.

Wahle, E., K. Mueller and E. Orr. (1985). Effect of DNA gyrase inactivation on RNA synthesis in Escherichia coli. J. Bacteriol. 162: 458-460.

Wang, J. C. (1971). Interaction between DNA and an E. coli protein $\omega$. J. Mol. Biol. 55: 523-533. 
Wang, J. C. (1974). Interactions between twisted DNAs and enzymes: the effects of superhelical turns. J. Mol. Biol. 87: 797-816.

Wang, J. C. (1979). Helical repeat of DNA in solution. Proc. Natl. Acad. Sci. USA. 76: 200-203.

Wang, J. C. (1985). DNA topoisomerases. Annu. Rev. Biochem. 54: $665-697$.

Wang, J. C. (1987). Recent studies of DNA topoisomerases. Biochem. Biophys. Acta. 909: 1-9.

Wang, J. C., M. D. Barkley and S. Bourgeois. (1974). Measurement of unwinding of lac operator by repressor. Nature. 251: 247.

Wasserman, S. A., J. H. White and N. R. Cozzarelli. (1988). The helical repeat of double-stranded DNA varies as a function of catenation and supercoiling. Nature. 334: 448-450.

Weaver, P. F., J. D. Wall and H. Gest. (1975). Characterization of Rhodopseudomonas capsulata. Arch. Microbiol. 105: 207-216.

Wellington, C. L. and J. T. Beatty. (1989). Promoter mapping and nucleotide sequence of the bchC bacteriochlorophyll biosynthesis gene from Rhodobacter capsulatus. Gene. 83: 251-261. 
White, J. H. (1969). Self-linking and the Gauss integral in higher dimensions. Am. J. Math. 91: 693.

White, J. H. and W. R. Bauer. (1988). Applications of the twist difference to DNA structural analysis. Proc. Natl. Acad. Sci. USA. 85: $772-776$.

White, J. H., N. R. Cozzarelli and W. R. Bauer. (1988). Helical repeat and linking number of surface-wrapped DNA. Science. 241: 323327.

Widom, J. and R. L. Baldwin. (1983). Tests of spool models for DNA packaging in phage lambda. J. Mol. Biol. 171: 419-437.

Willetts, N. S., A. J. Clark and B. Low. (1969). Genetic location of certain mutations conferring recombination deficiency in Escherichia coli. J. Bacteriol. 97: 244-249.

Wolberger, C., Y. C. Dong, M. Ptashne and S. C. Harrison. (1988). Structure of a phage 434 Cro/DNA complex. Nature. 335: 789-95.

Worcel, A. and E. Burgi. (1972). On the structure of the folded chromosome of Escherichia coli. J. Mol. Biol. 71: 127-147.

Wu, H.-Y., S. Shyy, J. C. Wang and L. F. Liu. (1988). Transcription generates positively and negatively supercoiled domains in the template. Cell. 53: 433-440. 
Wu, P., L. Song, J. B. Clendenning, B. S. Fujimoto, A. S. Benight and J. M. Schurr. (1988). Interaction of chloroquine with linear and supercoiled DNAs. Effect on the torsional dynamics, rigidity, and twist energy parameter. Biochemistry. 27: 8128-8244.

Yamamoto, N. and M. L. Droffner. (1985). Mechanisms determining aerobic or anaerobic growth in the facultative anaerobe Salmonella typhimurium. Proc. Natl. Acad. Sci., USA. 82: 2077-2081.

Yang, Z. and C. E. Bauer. (1990). Rhodobacter capsulatus genes involved in early steps of the bacteriochlorophyll biosynthetic pathway. J. Bacteriol. 172: 5001-5010.

Yanofsky, C. and I. P. Crawford. (1987). The tryptophan operon. Escherichia coli and Salmonella typhimurium: Cellular and molecular biology. Washington, D.C., American Society for Microbiology.

Yanofsky, C., T. Platt, I. P. Crawford, B. P. Nichols, G. E. Christie, H. Horowitz, M. VanCleemput and A. M. Wu. (1981). The complete nucleotid sequence of the tryptophan operon of Escherichia coli. Nucl. Acids Res. 9: 6647-6668.

Young, D. A., C. E. Bauer, J. C. Williams and B. L. Marrs. (1989). Genetic evidence for superoperonal organization of genes for 
photosynthetic pigments and pigment-binding proteins in Rhodobacter capsulatus. Mol. Gen. Genet.

Zhu, Y. S. and J. E. Hearst. (1988). Transcription of oxygen-regulated photosynthetic genes requires DNA gyrase in Rhodobacter capsulatus. Proc. Natl. Acad. Sci., USA. 85: 4209-4213.

Zsebo, K. M. and J. E. Hearst. (1984). Genetic-physical mapping of a photosynthetic gene cluster from $R$. capsulata. Cell. 37: 937-947. 

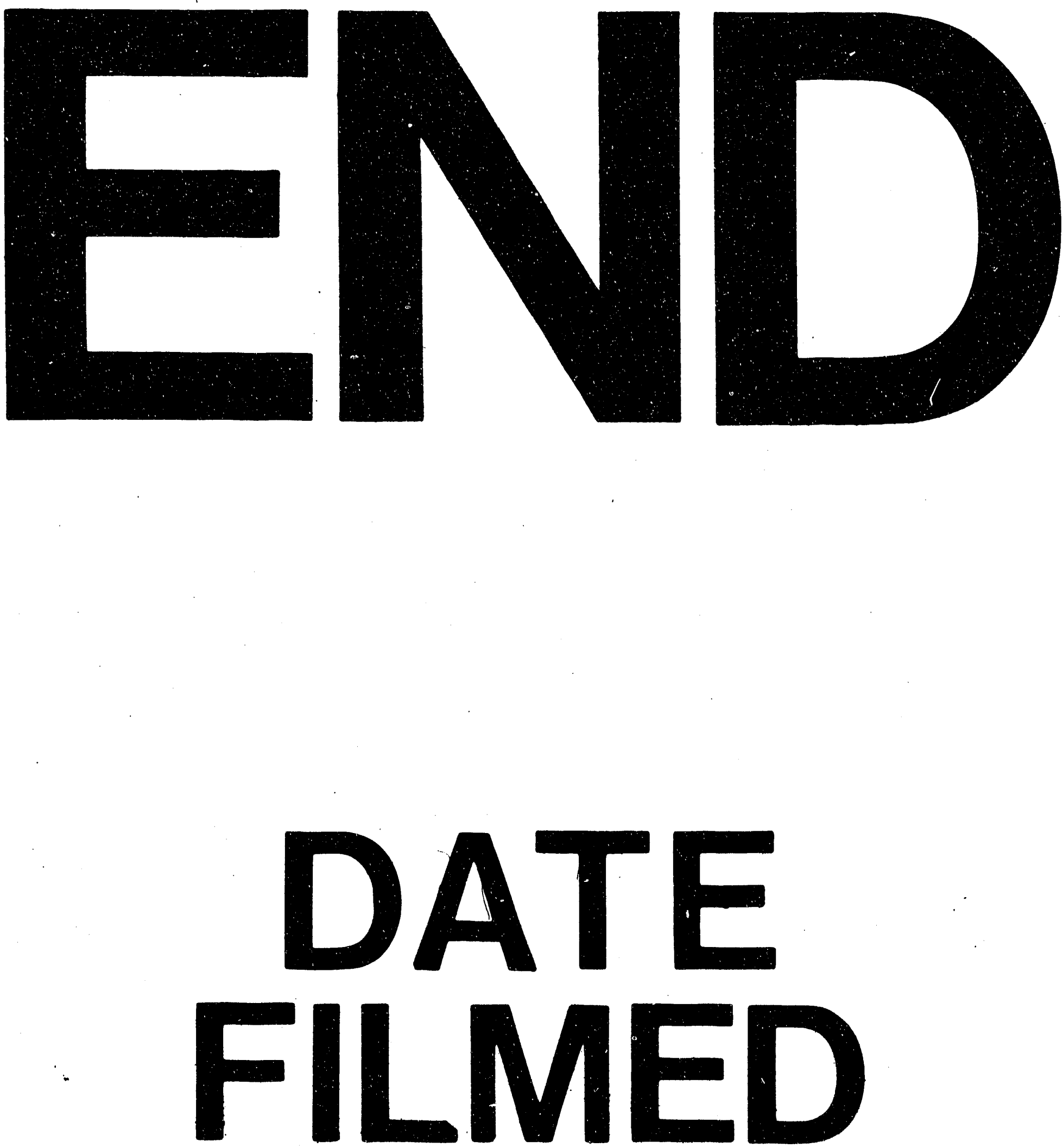

1

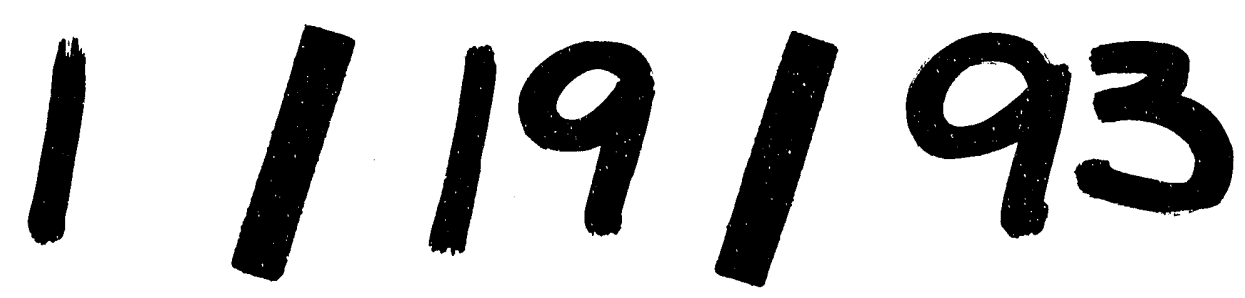


, 